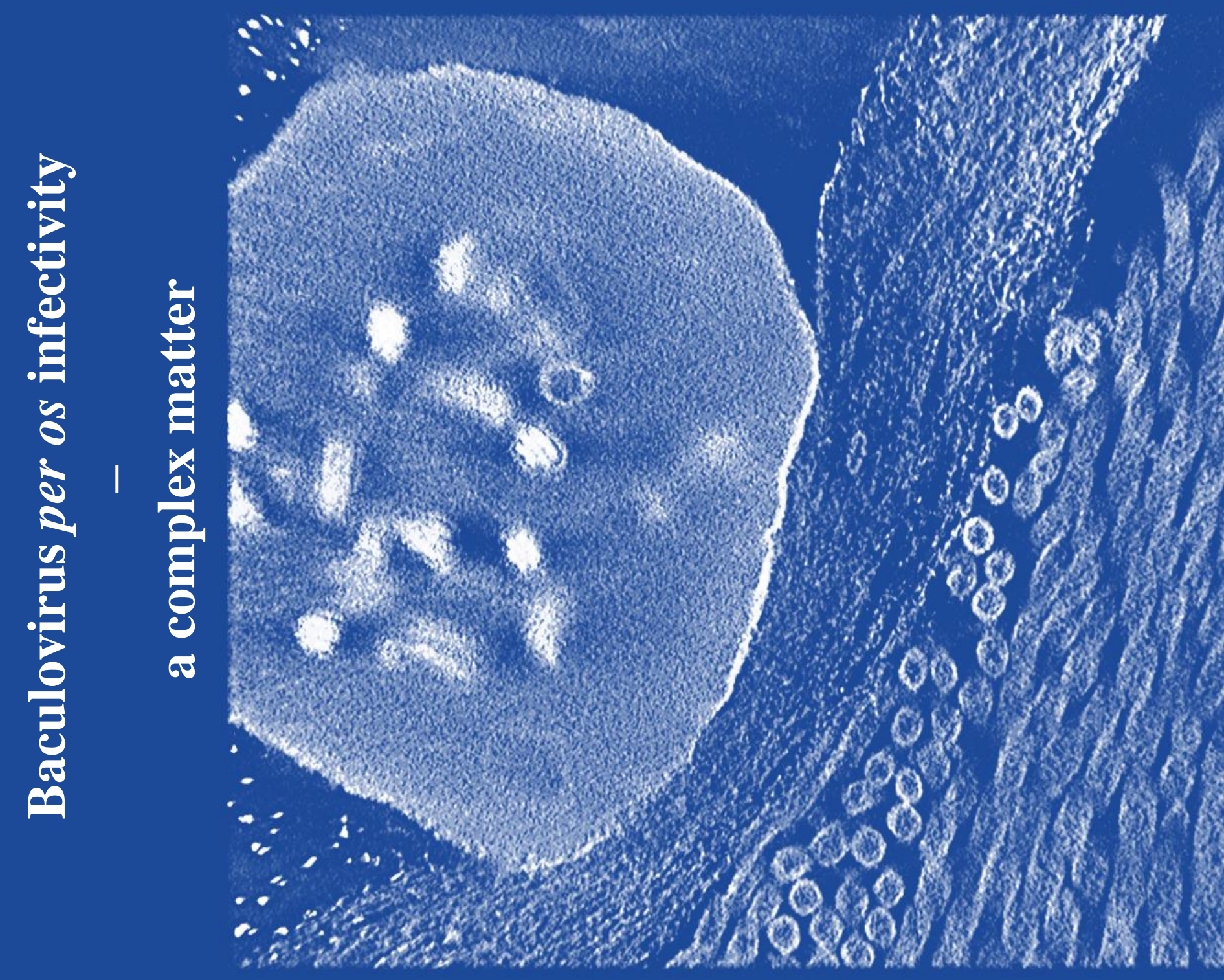

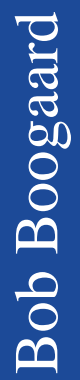

Baculovirus per os infectivity - a complex matter Bob Boogaard

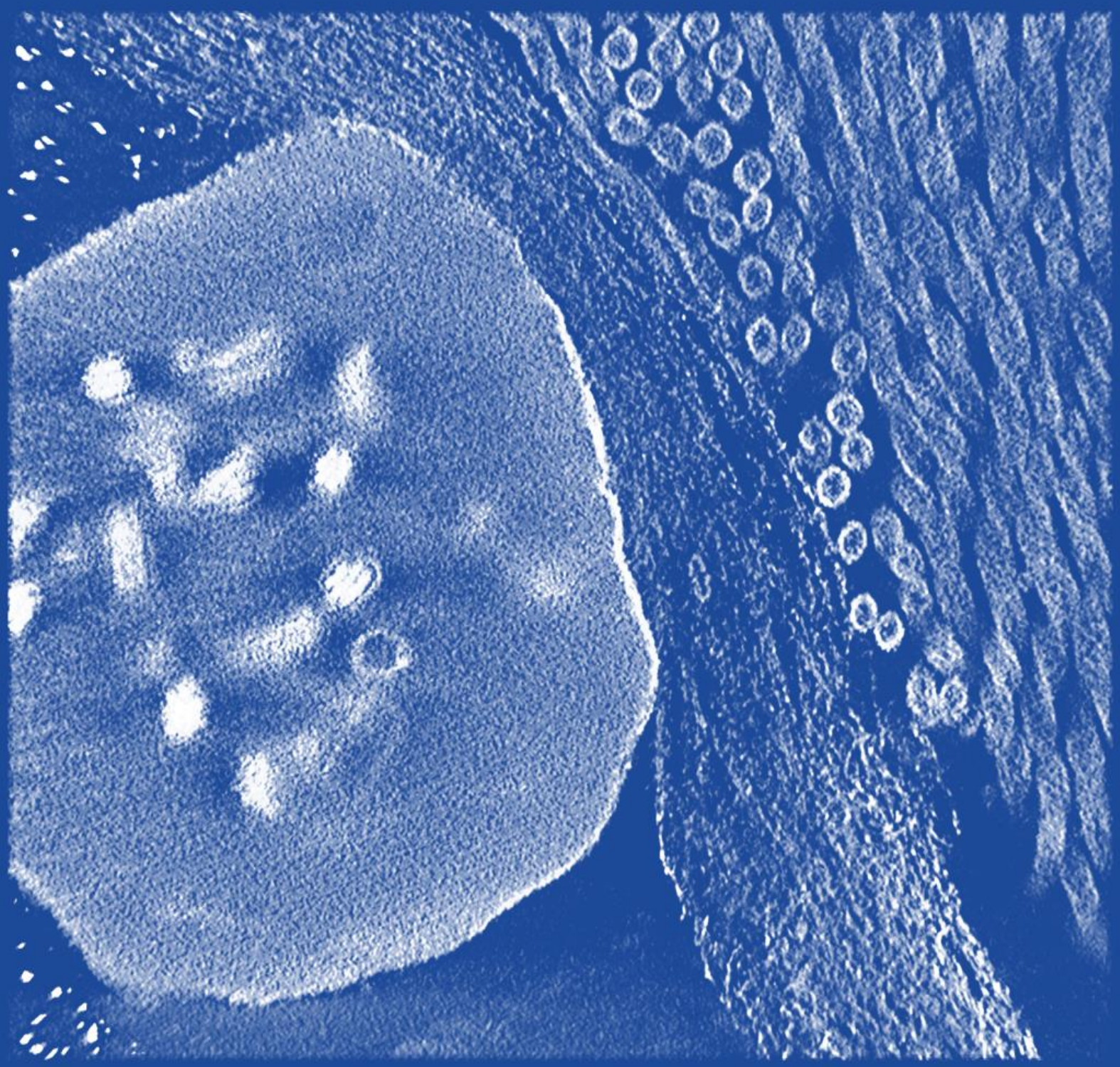




\section{Propositions}

1. Baculovirus per os infectivity factors act according to the slogan of the three musketeers: all for one, one for all (this thesis).

2. The use of only fluorescence dequenching assays to study membrane fusion leads to premature conclusions on virus entry (this thesis).

3. The origin of virus-like sequences, identified by metagenomic sequencing, should be elucidated before the claim of a newly identified virus can be deposited.

4. The use of recombinant DNA technology to increase herbicide resistance in soya bean plants is immoral.

5. The value of fundamental scientific research without direct societal relevance is underestimated.

6. The broadcast of the new "Dancing with the Stars" series increases the horizontal transmission of the dance-virus rather than its vertical transmission to the next generation.

Propositions belonging to the thesis, entitled

Baculovirus per os infectivity - a complex matter

Bob Boogaard

Wageningen, 15 January 2020 


\section{Baculovirus per os infectivity factors}

a complex matter 


\section{Thesis committee}

\section{Promotor}

Prof. Dr M.M. van Oers

Professor of Virology

Wageningen University \& Research

\section{Co-promotor}

Dr J.W.M. van Lent

Assistant professor, Laboratory of Virology

Wageningen University \& Research

\section{Other members}

Prof. Dr D. Weijers, Wageningen University \& Research

Dr C.J.M. Koenraadt, Wageningen University \& Research

Dr B.J. Bosch, Utrecht University

Dr K. Peng, Wuhan Institute of Virology, China

This research was conducted under the auspices of the Graduate School for Production Ecology and Resource Conservation. 


\title{
Baculovirus per os infectivity factors a complex matter
}

\author{
Bob Boogaard
}

\section{Thesis}

submitted in fulfilment of the requirements for the degree of doctor at Wageningen University

by the authority of the Rector Magnificus,

Prof. Dr A.P.J. Mol, in the presence of the

Thesis Committee appointed by the Academic Board to be defended in public

on Wednesday 15 January 2020

at 4 p.m. in the Aula. 
Bob Boogaard

Baculovirus per os infectivity - a complex matter, 157 pages

$\mathrm{PhD}$ thesis, Wageningen University, Wageningen, The Netherlands (2020)

With references, with summaries in English and Dutch

ISBN: 978-94-6395-213-2

DOI: https://doi.org/10.18174/506926 


\section{Table of contents}

Chapter 1 General introduction and thesis outline

Chapter 2 An advanced view on baculovirus per os infectivity factors

Chapter 3 Baculoviruses require an intact ODV entry complex to resist proteolytic degradation of per os infectivity factors by cooccluded proteases from the larval host

Chapter 4 The baculovirus AC108 protein is a per os infectivity factor and 45 a component of the ODV entry complex

Chapter 5 Functional analysis of baculovirus per os infectivity factors 3 61 and 9 by imaging the interaction between fluorescently labelled virions and isolated midgut cells

Chapter 6 The C-termini of baculovirus per os infectivity factors 1 and 2 mediate ODV oral infectivity by facilitating the binding of PIF0 and PIF8 to the core of the entry complex

Chapter 7 Reconstruction of the interactome of the baculovirus per os 101 infectivity factors

Chapter 8 General discussion

References

List of abbreviations

Summary - Samenvatting

Acknowledgements - Dankwoord

About the author

Account 



\section{Chapter 1}

General introduction and thesis outline 


\section{Introduction}

Baculoviruses are insect specific viruses that are used in agriculture as biological insecticide against insect pests (see review by Szewczyk et al., 2009). For example, the Helicoverpa armigera nucleopolyhedrovirus (HearNPV) is used in the cotton fields in China and India against the cotton bollworm (Helicoverpa armigera) (Mettenmeyer, 2002). In Brazil, soybean plants are protected against the velvet bean caterpillar (Anticarsia gemmatalis) with the Anticarsia gemmatalis multiple nucleopolyhedrovirus (AgMNPV) (Moscardi and Sosa-Gomez, 1992). The major advantage of using baculoviruses in agricultural pest management is the small ecological footprint as most baculoviruses infect only one or a few related insect species. The pest insects are infected at their larval stages when consuming virus contaminated plant material, after which the ingested viruses infect the epithelial cells in the larval midgut. The primary infection of the midgut is one of the host range determinants of baculoviruses (HaasStapleton et al., 2005) and is the first crucial step of the replication cycle. Despite the broad scale application of these viruses as biological insecticide, the underlaying mechanism of the midgut infection process is still enigmatic. The research presented in this thesis therefore studies the viral proteins that are essential for the virus entry into midgut epithelial cells.

\section{The baculovirus infection cycle}

Baculoviruses infect insect larvae via the oral route when a larva consumes food sources, which are contaminated with viral occlusion bodies (OBs). OBs consist of a protein matrix of crystallized polyhedrin or granulin, in which the virus particles are embedded (Fig. 1). The protein matrix protects the virus particles after the dispersal of OBs into the environment. These virus particles are called: occlusion derived viruses (ODVs). Upon ingestion of the OBs by an insect larva, the protein matrix dissolves in the highly alkaline environment of the midgut, resulting in release of the ODVs (Fig. 2a and b). The ODVs pass the peritrophic membrane that separates the gut contents from the epithelium

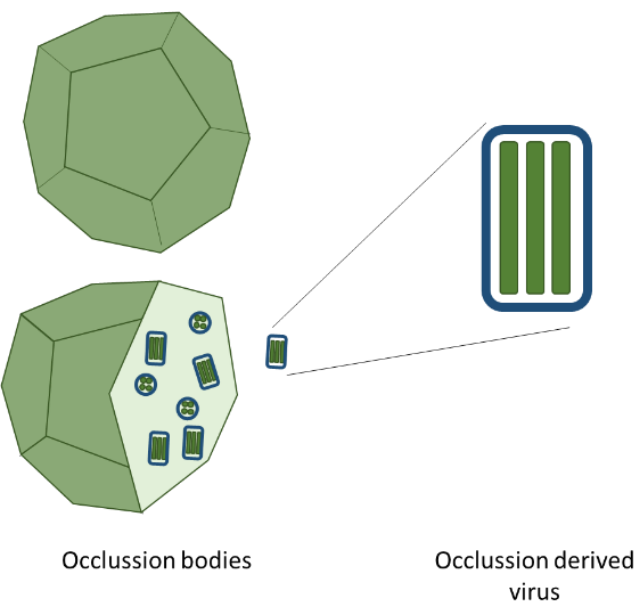

Figure 1: Baculovirus occlusion bodies with embedded virions. The occlusion bodies consist of crystalized polyhedrin or granulin, in which the occlusion derived viruses (ODVs) are embedded. The ODVs contain multiple nucleocapsids (in green) and is surrounded by an envelope that is derived from the host cell inner nuclear membrane (in blue). 


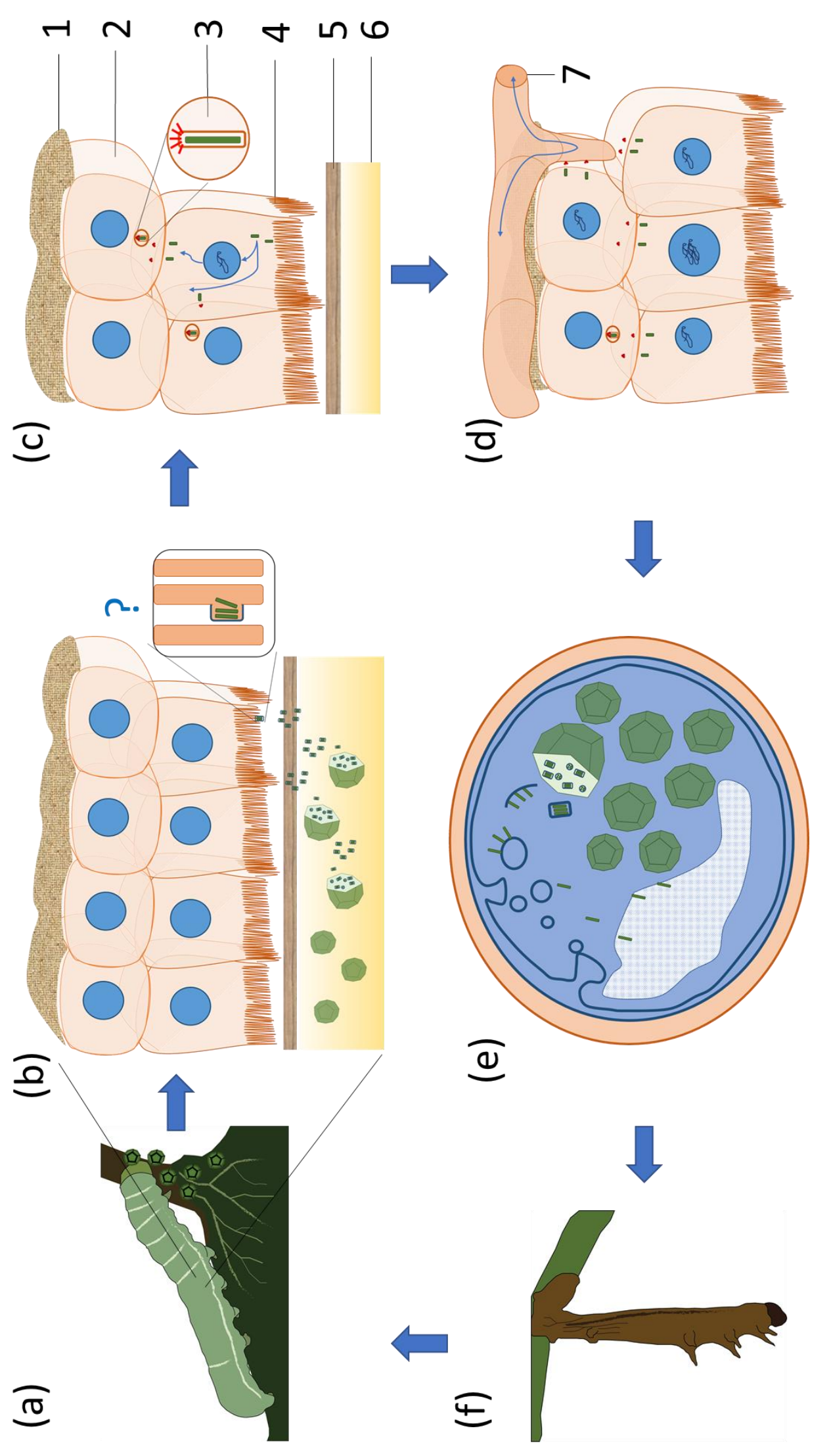

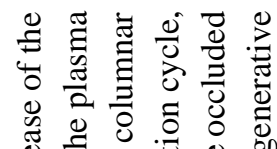

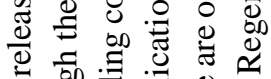

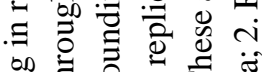

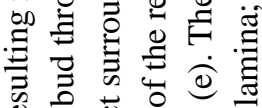

造

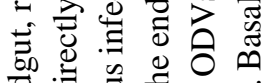

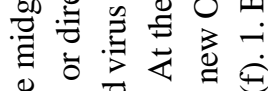

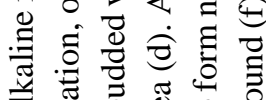

亲氜

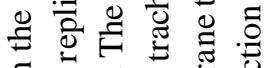

:

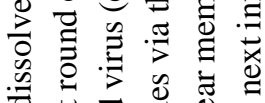

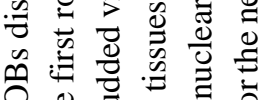

Oे

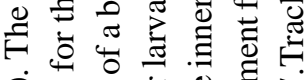

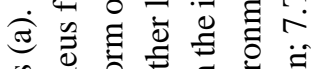

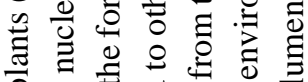

줄

可

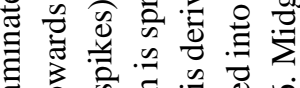

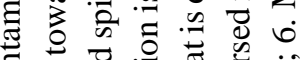

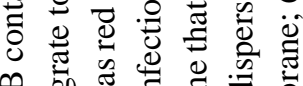
oิ

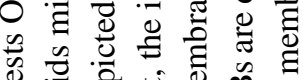
总

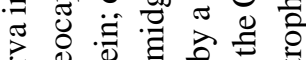

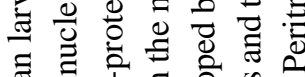

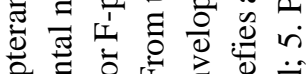

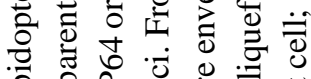

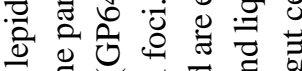

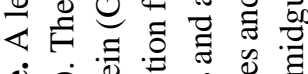

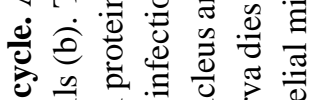

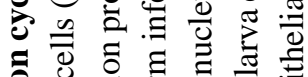

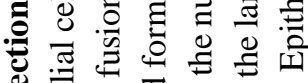

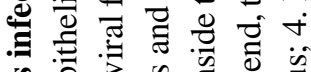

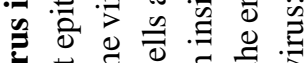

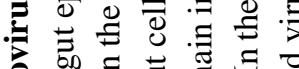
c 歌

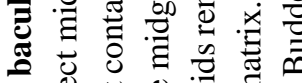

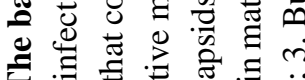
政

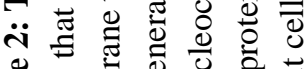
言资

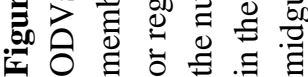


(Hegedus et al., 2009), and infect the midgut epithelium cells (Fig 2b). Mainly the epithelial cells in the anterior region of the midgut are infected by the ODVs (Javed et al., 2016).

The ODVs bind and fuse with the microvilli at the apical side of the epithelium cells, followed by release of the nucleocapsids into the cytosol (Kawanishi et al., 1972, Granados et al., 1981, Horton and Burand, 1993). At the start of this PhD research, eight different ODV envelope proteins were known to be crucial for midgut infection (Faulkner et al., 1997, Kikhno et al., 2002, Pijlman et al., 2003, Ohkawa et al., 2005, Fang et al., 2009, Sparks et al., 2011a, Nie et al., 2012, Zhu et al., 2013). These proteins are called per os infectivity factors or PIFs. Three PIFs had been found to be important for the binding of ODVs to the midgut epithelium (HaasStapleton et al., 2004, Ohkawa et al., 2005) and the biological role of the other PIFs in midgut entry was unknown. Six of the PIFs had been shown to participate in the formation of a multimeric complex (Peng et al., 2010, 2012), which in this thesis is named the "ODV entry complex". This thesis focuses on the involvement of this protein complex in primary infection of the midgut cells (Fig. 2b). The biochemical and functional characteristics of the PIFs and their involvement in formation of the ODV entry will be further introduced in Chapter 2.

After the ODV envelope has fused with the microvillar plasma membrane, the parental nucleocapsids are released and migrate to the nucleus for the first round of replication and production of progeny capsids (Fig. 2c). The infected midgut cells subsequently produce a second virion phenotype, the budded viruses (BVs). These are formed when the progeny nucleocapsids bud through the host cell plasma membrane that contains the BV fusion protein (either GP64 or the F-protein) (Fig. 2c). BVs infect the adjacent midgut cells, which ultimately leads to the formation of infection foci in the midgut epithelium (Engelhard et al., 1994, Flipsen et al., 1995, Javed et al., 2016). There is experimental evidence that suggests that some parental nucleocapsids directly migrate to the underlaying regenerative cells as those were found infected before virus replication in the epithelial cells became apparent (Flipsen et al., 1995). The infection of the regenerative cells could be important to prevent clearing of the infection by sloughing of the infected epithelial cells (Flipsen et al., 1993). The direct transfer of some parental nucleocapsids might be mediated by the early expression of the BV fusion protein GP64 (Washburn et al., 2003), which is also required for the formation of the infection foci (Javed et al., 2016).

The virus infection is systemically spread from the midgut to other larval tissues by BVs via trachea and the hemolymph (Fig. 2d) (Engelhard et al., 1994, Chapter 4 Thesis J.T.M. Flipsen, 
1995), where the infected cells also start to produce BVs. Later in the infection cycle, the progeny virus production is switched from BVs to ODVs, in which the nucleocapsids are retained inside the host cell nucleus and are enveloped by a PIF-containing membrane that is derived from the inner nuclear membrane (Fig. 2e) (Williams and Faulkner, 1997). The ODVs are embedded in crystallized polyhedrin or granulin to form new OBs for the next infection round. The OBs are dispersed into the environment after liquefaction of the larva by the activity of viral cathepsin and chitinase (Fig. 2f) (Hawtin et al., 1997).

As previously mentioned, this $\mathrm{PhD}$ thesis aims to study how PIF proteins, and the multimeric complex they form, achieve entry into the midgut epithelial cells, to initiate a new infection round in insect larvae. PIF proteins are highly conserved among the baculoviruses and some are even found in related insect viruses, suggesting that this entry mechanism has already evolved millions of years ago (Thézé et al., 2011). Furthermore, the PIFs operate under highly alkaline conditions, in contrast to other known viral entry proteins that act under acidic or neutral conditions. The exceptional conditions for virus entry and the formation of a multimeric complex suggest that we are dealing with an unknown entry pathway, of which the molecular mechanism has yet to be elucidated.

\section{Outline of the thesis}

At the start of this thesis research, eight PIFs had been identified, of which six were known to be present in what we now call the ODV entry complex. The aim of this thesis was to determine the composition of the baculovirus entry complex in more detail and to understand the requirements for complex formation. Furthermore, the first studies were conducted to understand the biological function of complex formation for the entry into midgut cells. For this, a series of pif mutant viruses were generated for the prototype alphabaculovirus Autographa californica multiple nucleopolyhedrovirus (AcMNPV) and analysed for their ability to assemble the complex and to orally infect Spodoptera exigua larvae. For some mutants, fluorescently labelled ODVs were generated and their infection properties were monitored on isolated larval midgut cells by confocal microscopy.

Most data on PIF proteins, described in earlier literature, were obtained not knowing that these proteins form a complex. Chapter 2 therefore reviews PIF literature and the function of PIF proteins in view of their presence in the complex. 
When the first pif mutant viruses were generated for our initial studies on the ODV entry complex, it was found that besides the PIF protein targeted by mutagenesis, also the nontargeted PIFs were not detectable when the OBs were produced in S. exigua larvae. However, the non-targeted PIFs were still found when the mutant viruses were produced in cultured insect cells. It turned out that the co-occluded host proteases in larval-derived OBs, which have been described by Eppstein and Thoma (1975), Payne and Kalmakoff (1978) and Wood (1980), severely affected the integrity of the PIFs in these mutant viruses. Chapter 3 describes how these proteases affect the PIFs and the complex when the viruses are produced by their (natural) caterpillar host. For this, OBs of bacmid derived AcMNPV and various pif deletion mutants are produced in S. exigua larvae and cultured insect cells, and the individual PIFs and the complex are compared by western blot analyses with antisera against various PIF proteins.

Earlier proteomic analysis (Peng et al., 2012) identified the AcMNPV protein AC108 as a putative PIF1 interaction partner. In Chapter 4, AC108 is studied for its function in oral infectivity and presence in the entry complex by using ac108 deletion and repair viruses. The function of AC108 in binding to and entry into midgut epithelium cells is subsequently studied using fluorescently labelled ODVs of the ac108 mutant and wild type virus. Interactions of ODVs with isolated midgut epithelium cells are followed as function of time by life imaging with a confocal microscope (Chapter 5). This chapter also reports observations with fluorescent ODVs of a pif3 deletion mutant that is still able to bind and fuse with the epithelium cells according to literature, but nevertheless fails to establish infection in the midgut (Ohkawa et al., 2005).

PIF1 and PIF2 are essential for formation of the entry complex and are involved in interaction with the midgut epithelium of the host (Ohkawa et al., 2005, Peng et al., 2010). To determine whether these PIFs interact with the midgut directly or whether ODV binding is mediated via the interaction with other PIFs, a series of pif1 and pif2 truncation mutant viruses are analysed for oral infectivity and complex formation (Chapter 6). In addition, the requirements for complex formation are analysed by coimmunoprecipitation studies with the truncation mutants and various pif deletion mutant viruses.

PIF5 is the only PIF that is not part of the ODV entry complex (Peng et al., 2012), while being important for the oral infectivity (Harrison et al., 2010, Sparks et al., 2011). Furthermore, some non-PIF proteins are also known to influence the infectivity of ODVs, although more subtle (Xiang et al., 2011, Sparks et al., 2011b, Li et al., 2018, Lapointe et al., 2004, Zhang et al., 
2005). To determine how the ODV entry complex relates to the other ODV envelope proteins, a proteomic analysis is performed after immunoprecipitation of three different components of the entry complex (PIF6, 8 and 9), and PIF5 (Chapter 7).

In Chapter 8, the results are discussed in a broader perspective. The complexity of midgut infection by ODVs is discussed and the entry complex is compared with two other known viral entry complexes. Furthermore, a model of how PIFs mediate infection of the midgut epithelial cells is proposed. 


$$
-8-
$$




\title{
Chapter 2
}

\section{An advanced view on baculovirus per os infectivity factors}

Bob Boogaard, Monique M. van Oers, Jan W. M. van Lent

\begin{abstract}
Baculoviruses are arthropod-specific large DNA viruses that orally infect the larvae of more than six hundred different insect species of the orders Lepidoptera, Hymenoptera and Diptera. Insect larvae become infected when they eat a food source that is contaminated with viral occlusion bodies (OBs). These OBs contain occlusion-derived viruses (ODVs), which are released upon ingestion of the OBs and infect the endothelial midgut cells. At least nine different ODV envelope proteins are essential for this oral infectivity and these are denoted as per os infectivity factors (PIFs). Seven of these PIFs form a complex, consisting of PIF1, 2, 3 and 4 that form a stable core complex, and PIF0 (P74), PIF6 and PIF8 (P95) that associate with this complex with lower affinity than the core components. The existence of a PIF complex and the fact that the pif genes are conserved in baculovirus genomes suggests that PIF-proteins cooperatively mediate oral infectivity rather than as individual functional entities. This review therefore discusses the knowledge obtained for individual PIFs in light of their relation with other members of the PIF complex.
\end{abstract}

This chapter has been published in modified form as:

Boogaard, B., van Oers, M. M., \& van Lent, J. W. M. (2018). An advanced view on baculovirus per os infectivity factors. Insects, 9, 84 . 


\section{Introduction}

The Baculoviridae form a family of arthropod-specific large double stranded DNA viruses that infect the larvae of more than six hundred different insect species (Williams et al., 2017). The family is divided into four genera, which reflects the co-evolution of these viruses and the insects they infect. Viruses classified in the genera Alphabaculovirus and Betabaculoviruses infect the larvae of lepidopteran insect species. Gammabaculoviruses infect larvae of hymenopteran insect species and the single known virus classified as Deltabaculovirus infects Culex mosquito larvae (Reviewed by Williams et al., 2017). The majority of baculoviruses that infect lepidopteran larvae have narrow host ranges, i.e. a specific baculovirus infects the larval stage of only one or a few host species. Thus, several baculoviruses have been successfully used as biological control agents for particular species of Lepidoptera that are economic pests in agriculture and forestry (Reviewed by Szewczyk et al.,2009). For example, Anticarsia gemmatalis multiple nucleopolyhedrovirus (AgMNPV) is used to protect soybean plants against the velvet bean (Anticarsia gemmatalis) caterpillar in Brazil (Moscardi et al., 2007).

The name baculovirus derives from the latin word baculum, which refers to the rod-shaped morphology of their nucleocapsids. All baculoviruses have large, circular double stranded DNA genomes that range from 80-180 kbp in size and encode 90-180 genes (Williams et al., 2007). Baculoviruses form two different virion phenotypes, the budded viruses (BVs) and the occlusion-derived viruses (ODVs). The BV virion type is responsible for systemic spread of the infection within the larvae. Each BV consists of a single nucleocapsid surrounded by an envelope derived from the plasma membrane of a host cell. These BVs display a fusion protein to allow cell entry (either GP64 or F protein). The ODVs in contrast are formed in the nuclei of infected cells and consist either of a single (SNPV) or multiple (MNPV) nucleocapsids. ODVs are surrounded by an envelope that is derived from the inner nucleus membrane and contains a number of proteins encoded by baculovirus genes (see below). ODVs are also embedded in a crystalline matrix, forming occlusion bodies (OBs). The OBs produced by Alpha-, Gamma-, and Deltabaculoviruses are composed of a viral protein named polyhedrin, while the OBs produced by Betabaculoviruses are comprised of a different viral protein, named granulin. The structure of the OBs protects the ODVs against detrimental influences from the environment. ODVs are responsible for the horizontal transmission of the virus. When insect larvae eat OBcontaminated food, the OBs disintegrate in the highly alkaline milieu of the larval midgut, which releases ODVs into the gut lumen. ODVs then bind to the microvilli of midgut columnar cells and the viral envelope fuses with the cell membrane, releasing nucleocapsids into the cell. 
This process of virus entry is mediated by a specific set of proteins in the ODV envelope, the so-called per os infectivity factors (PIFs). For Autographa californica multiple nucleopolyhedrovirus (AcMNPV), Peng and co-workers showed that PIFs form a large protein complex, suggesting that these proteins mediate midgut entry of baculovirus ODVs in a cooperative manner (Peng et al., 2010, 2012). In this review, we summarize current knowledge of PIF-protein structure and function.

\section{Definition and general features of PIFs}

Per os infectivity factors are proteins that are essential for primary infection of the midgut columnar cells as deletions of pif-genes inhibit the oral infectivity of ODVs. Using AcMNPV, nine ODV-envelope proteins have been identified as PIF (Table 1). Since PIFs are not needed for cell to cell spread of the virus within the insect, the infectivity of BVs is not affected after deletion of a pif-gene. As a consequence, pif deletion mutants show wildtype characteristics in cultured insect cells. Larvae, however, can only be infected with such mutants when the midgut is bypassed by injecting BVs into the hemocoel (Faulkner et al., 1997, Kikhno et al., 2002, Pijlman et al., 2003). All known PIF proteins identified to date are encoded by baculovirus core genes, i.e. they are present and highly conserved in all sequenced baculovirus genomes across the four genera in the family Baculoviridae (Javed et al., 2017, Wang et al., 2017) Some PIFs are also found in other invertebrate DNA viruses like nudiviruses, bracoviruses, hytrosaviruses and nimaviruses (Reviewed by Wang et al., 2017). This high level of conservation suggests that PIFs mediate an ancient virus entry pathway.

A feature shared by all PIFs is the presence of highly conserved cysteine residues (Table 1). This high-level of cysteine conservation indicates that sulfate bridges are important for the structural and functional properties of PIFs. In silico analysis, using the Phobius algorithm

Table 1. Identified PIFs in AcMNPV

\begin{tabular}{ccccc}
\hline PIF & ORF & Size (kDa) & Nr. Of Cys. & Reference \\
\hline PIF0 & $a c 138$ & 74 & 6 & Faulkner et al. 1997 \\
PIF1 & $a c 119$ & 60 & 24 & Kikhno et al., 2002 \\
PIF2 & $a c 22$ & 44 & 14 & Pijlman et al., 2003 \\
PIF3 & $a c 115$ & 23 & 12 & Ohkawa et al., 2005 \\
PIF4 & $a c 96$ & 20 & 2 & Fang et al., 2009 \\
PIF5 & $a c 148$ & 56 & 6 & Harrison et al., 2010 \\
PIF6 & $a c 68$ & 16 & 1 & Nie et al., 2012 \\
PIF7 & $a c 110$ & 7 & 1 & Liu et al., 2016 \\
PIF8 & $a c 83$ & 96 & 13 & Zhu et al., 2013 \\
\hline
\end{tabular}



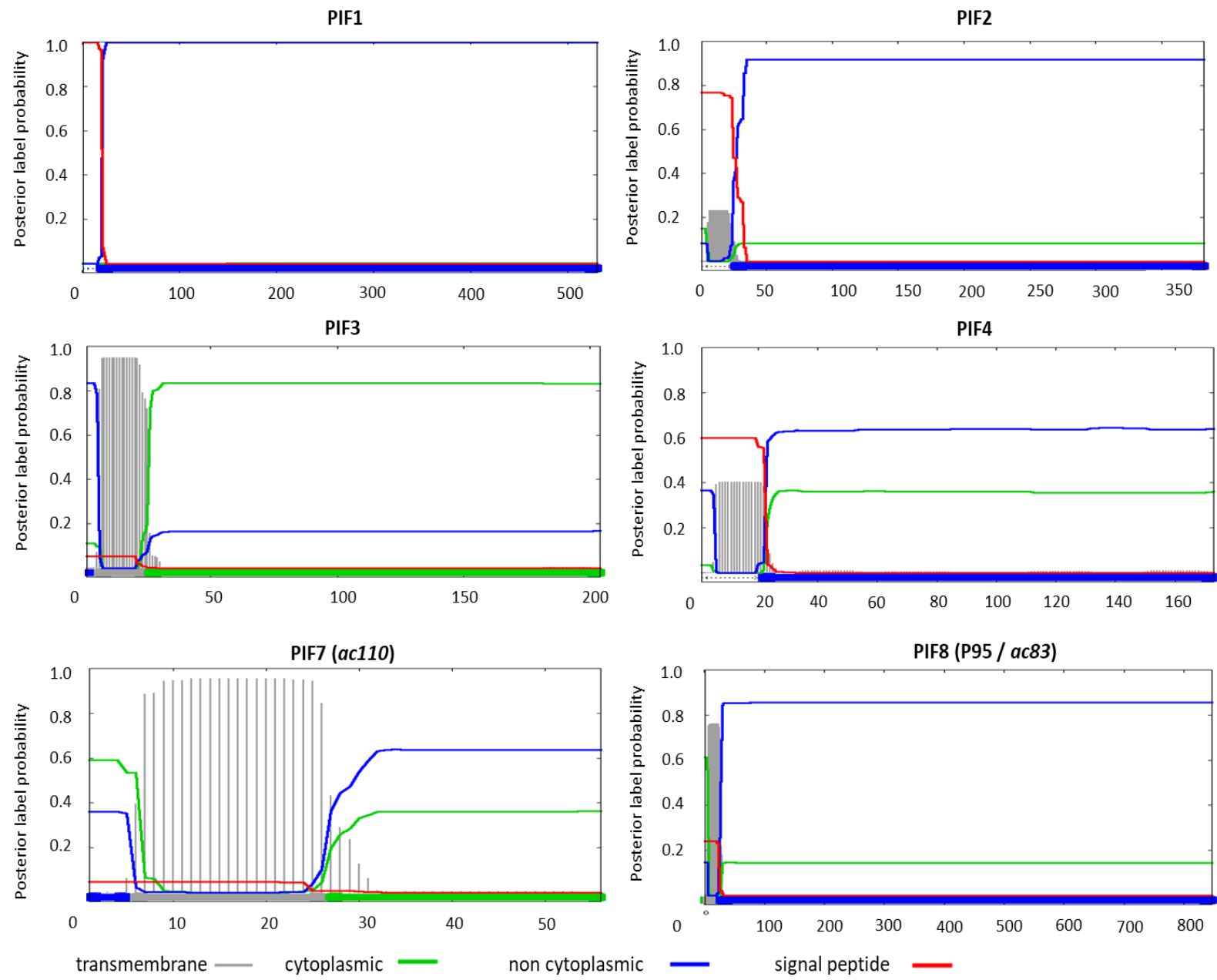

Figure 1. Prediction of the presence of transmembrane domains and signal peptides in per os infectivity factors (PIFs) and orientation of these proteins in an infected host-cell with the Phobius algorithm (Käll et al., 2004). On the $y$-axis: the probability for the presence of transmembrane domains (grey), cytoplasmic (green) or non-cytoplasmic (blue) orientation of the amino acid sequence and presence of a signal peptide (red). The final result of the prediction is summarized by the bar at the bottom with the indicated colours. The $x$-axis shows the number of the amino acids of that specific sequence. PIF1, PIF2, PIF3, PIF4, PIF7 (ac110) and PIF8 (P95/ac83) are predicted to have an N-terminal signal peptide or transmembrane domain.

(Käll et al., 2004) to find transmembrane domains and signal peptides, revealed N-terminal signal peptides and/or transmembrane helices in six out of the nine PIFs (PIF1 to 4, PIF7 and 8) (Fig. 1). For ODV-E66, another ODV-envelope protein, such an N-terminal domain was shown to be important for its translocation to the inner nuclear membrane (Braunagel et al., 2004). This domain was therefore called the inner nuclear membrane sorting motif (INM-SM) and consists of a hydrophobic domain of about 18 amino acids followed by at least one positively charged amino acid within 4-8 amino acids from the C-terminal end of the hydrophobic sequence. The predicted N-terminal transmembrane domain of the abovementioned PIFs also meets these criteria and the importance of this domain for routing to the inner nuclear membrane has been confirmed for PIF3 and PIF8 (Fig. 2) (Li et al., 2007, Zhu 


\begin{tabular}{|c|c|}
\hline \multicolumn{2}{|c|}{ AcMNPV (E2) } \\
\hline ODV-E66: & 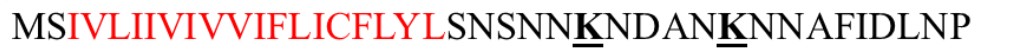 \\
\hline PIF1: & MHFAIILLFLLVIIAIVYTYVDLIDV $\underline{H H}$ EEV $\underline{\mathbf{R} Y P I T V F D N ~}$ \\
\hline PIF2: & MYRVLIFFLFVFYIVYQPFYQAYL $\underline{H}$ IG $\underline{H} A Q Q D Y N D T L D$ \\
\hline PIF3: & MLNFWQILILLVIILIVYMYTFK్KVQㅌFILQDAYMHINEL \\
\hline PIF4: & MLSIMLAIVFVLFVLIYLIISI $\underline{\bar{N}} \underline{H} \underline{H}$ PLLHIETLIQDFN \\
\hline F7. & MKYFLSATFLIIVFMYLMYFCISIVVNNA $\underline{\bar{H}}$ VQQDLFY $\underline{H} Y \mathrm{~N}$ \\
\hline г & AMSGVMLLMLAIFLIIAFTLMYLAIYFEFDETTFT \\
\hline
\end{tabular}

Figure 2: Comparison of the N-terminal transmembrane domains of PIF1, PIF2, PIF3, PIF4, PIF7 and PIF8 with the inner-nuclear membrane sorting motif of ODV-E66. In red: the hydrophobic region; in bold and underlined: the positively charged amino acids lysine $(\mathrm{K})$ and arginine $(\mathrm{R})$; in italic and underlined the amino acid histidine $(\mathrm{H})$ which is only positively charged when protonated.

et al., 2013). Similar N-terminal regions were not predicted for PIF0, PIF5 and PIF6 (Fig. 3). These proteins rather have hydrophobic domains at their C-termini, suggesting an alternative way for routing to the inner nuclear membrane and the ODV-envelope.

\section{The AcMNPV PIF-complex}

As shown by Peng et al., 2010, 2012, AcMNPV forms a macromolecular complex in the ODV envelope. So far, seven out of nine PIF proteins have been identified as components of this complex. This so-called ODV entry complex consists of a stable core, formed by PIF1, 2, 3 and 4, to which PIF0, 8 are more loosely associated. PIF6 is also a loosely associated component of this complex, as will be shown in Chapter 3. The core of the complex is characterised as stable,
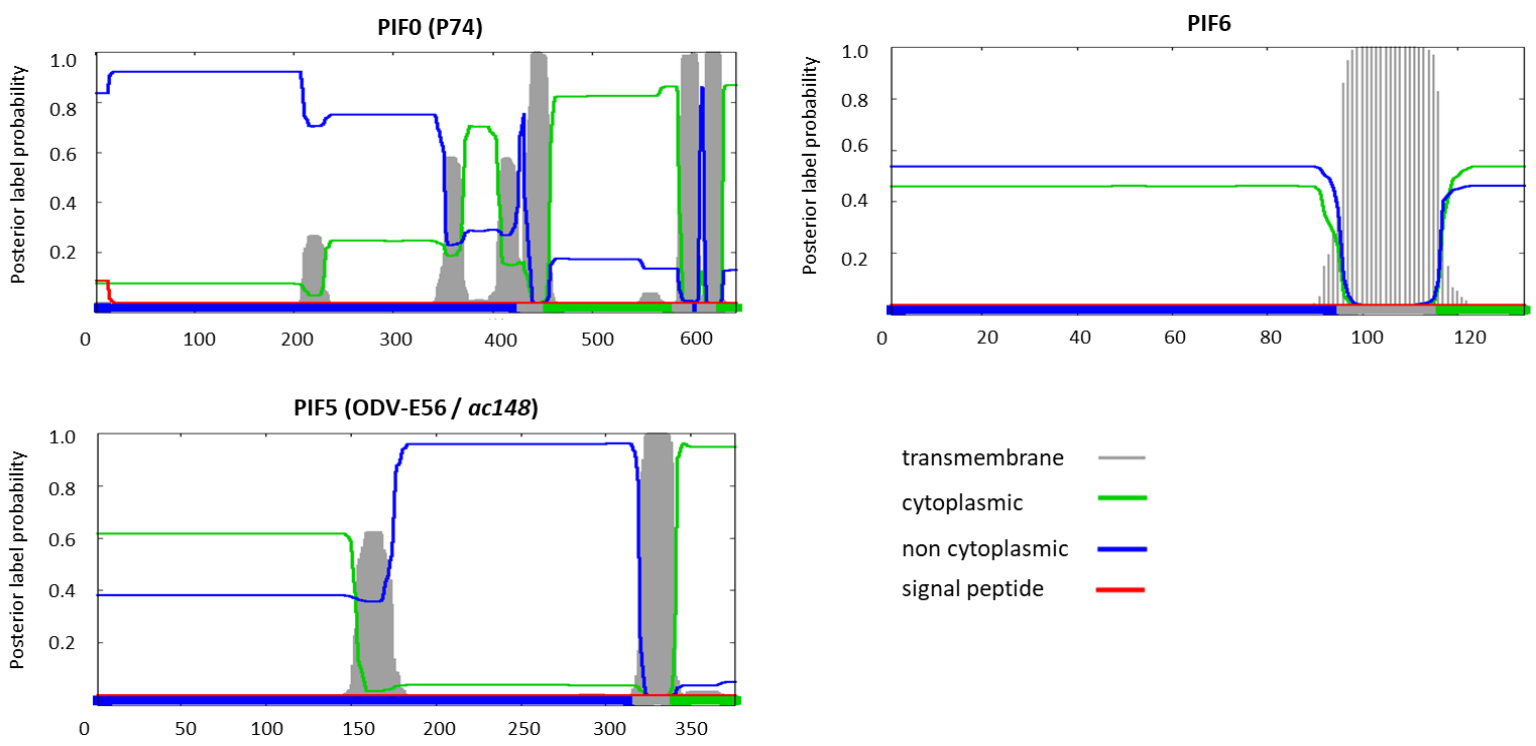

Figure 3: Prediction of the presence of transmembrane domains and signal peptides in PIF0, PIF5 and PIF6 and orientation of these proteins in an infected host-cell with the Phobius algorithm (Käll et al., 2004). On the y-axis: the probability for the presence of transmembrane domains (grey), cytoplasmic (green) or non-cytoplasmic (blue) orientation of the amino acid sequence and presence of a signal peptide (red). The final result of the prediction is summarized by the bar at the bottom with the indicated colours. The $\mathrm{x}$-axis shows the number of the amino acids of that specific sequence. 
since it appears as a $170 \mathrm{kDa}$ band in western blot analysis when ODVs were partially denatured by SDS and reducing agents and incubation at $50^{\circ} \mathrm{C}(\mathbf{F i g} . \mathbf{4 a})$. When under these conditions the temperature was increased to $95^{\circ} \mathrm{C}$, the core complex completely dissociated into PIF monomers (Peng et al., 2010). PIF1, 2 and 3 are essential for formation of the core complex, since the complex was not detected when any of the corresponding genes was deleted (Peng et al., 2010). After deletion of pif4, however, a smaller complex consisting of PIF1, 2 and 3 was found by western blot analysis (Peng et al. , 2012).

When ODVs were analysed under non-denaturing conditions by blue native-PAGE, the entry complex had an apparent molecular mass of $480 \mathrm{kDa}$ (Fig. 4b, left panel). This large multimolecular complex contains, besides the components of the core (PIF1, 2, 3 and 4), also PIF0, 6 and 8 (Peng et al., 2012, Chapter 3). ODV-envelope protein AC110 was recently assigned as PIF7 (Liu et al., 2016). To find out whether this PIF is also a component of the large entry complex, we analysed a virus with an HA-tagged PIF7 in the ODV-envelope under nondenaturing conditions as described above. With anti-HA antibodies, we were able to detect the same $480 \mathrm{kDa}$ band as found with PIF1 antiserum (Fig. 4b, right panel). This indicates that PIF7 is a component of the entry complex, which brings us to a total amount of eight complexassociated PIFs (Fig. 5). Apparently, PIF0, 6, 8 (and maybe also PIF7) associated to the core

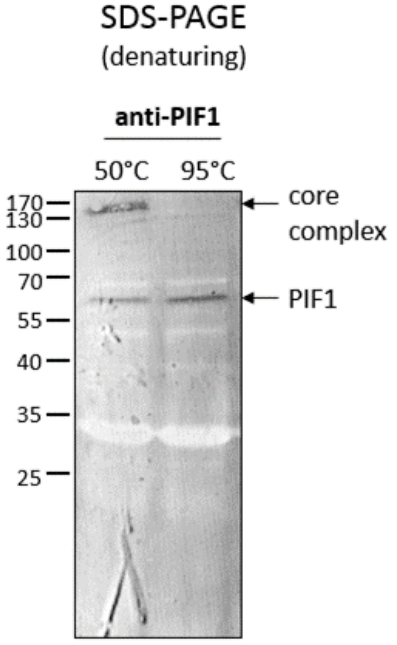

(a)

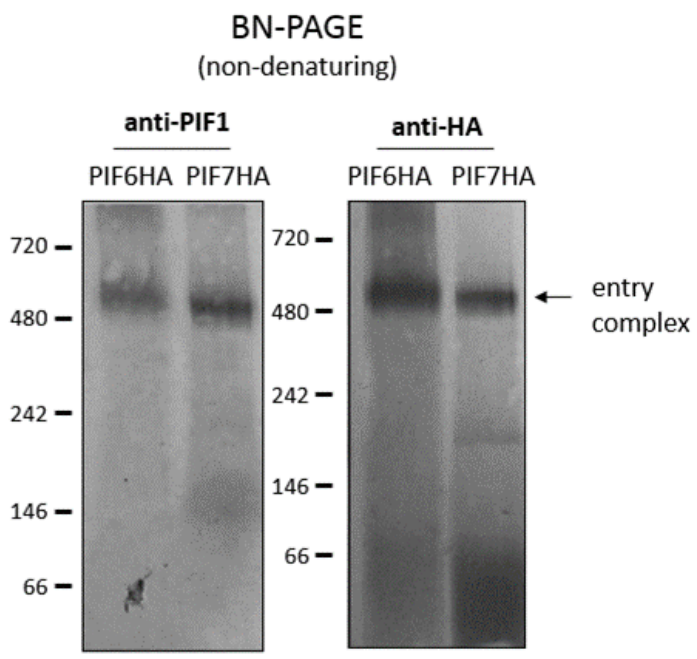

(b)

Figure 4: Detection of the stable core and the entry complex as a whole. When isolated ODVs were analysed under denaturing conditions by incubation in Laemmli buffer at $50{ }^{\circ} \mathrm{C}$, the core complex was found as a $170 \mathrm{kDa}$ band by western blot with PIF1 antiserum. Under these conditions, also PIF1 monomers were detected at $60 \mathrm{kDa}$. When the ODVs were incubated at $95^{\circ} \mathrm{C}$, the core complex dissociated and only PIF1 monomers were found (a). When isolated ODVs were analysed under non-denaturing conditions (b), the entry complex was detected as a band of approximately $480 \mathrm{kDa}$ with antiserum against PIF1 (left panel) and with anti-HA antibodies against HAtagged PIF6 (right panel). When analysing ODVs that contain HA-tagged PIF7 with anti-HA antibodies, the entry complex was also found, indicating that PIF7 is part of this complex (right panel). 


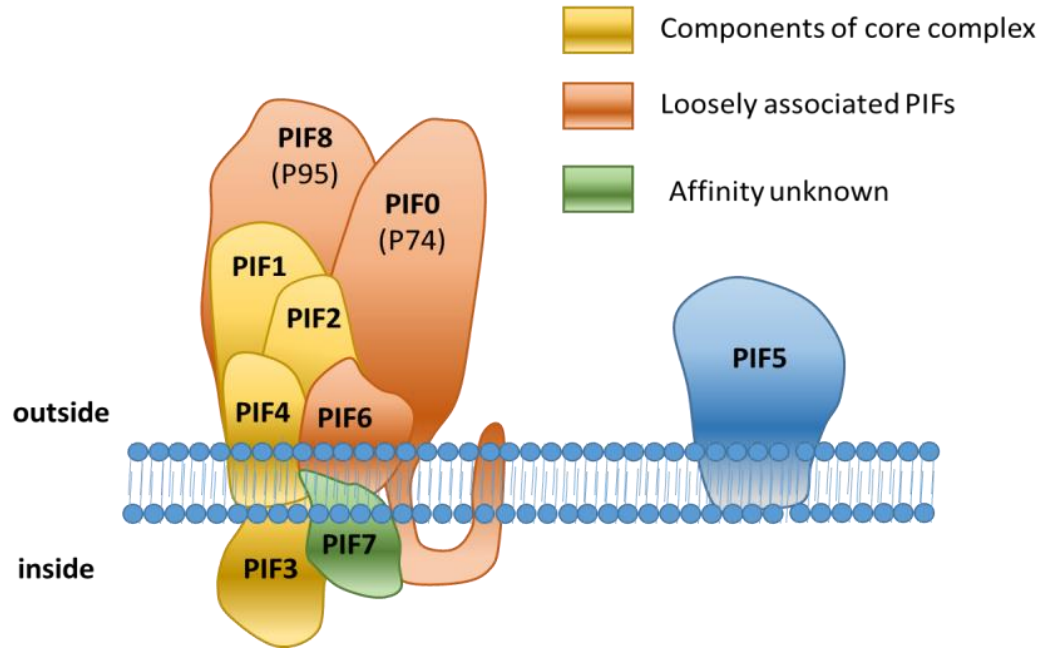

Figure 5: Model of the ODV entry complex. In yellow, the components of the core complex (PIF1, 2, 3 and 4); and in red, the PIFs that associate to the entry complex with lower affinity (PIF0, 6 and 8). PIF7 is depicted in green as this protein was detected in the entry complex under non-denaturing conditions but the affinity of this PIF for the complex is not known. PIF5 does not make part of the entry complex and is depicted in blue.

with lower affinity as these PIFs dissociated after (partial) denaturation by treatment with SDS and reducing agents, in contrast to the core components. Only PIF5 appears not to be present in the entry complex and this was confirmed by co-immune precipitations with PIF1 antiserum, in which this PIF was not found as interaction partner of PIF1 (Peng et al., 2012). This raises the question whether PIF5 functions independently from the entry complex during primary infection of the midgut. This co-immunoprecipitation study however, resulted in coprecipitation of PIF2, 3, 4, 0, 6 and 8, providing additional evidence for their presence in the entry complex. PIF7 was not found as an interacting partner in this study. AC5 and AC108 also precipitated with PIF1 (Peng et al., 2012). AC5 has recently been shown to associate with OBs and it seems not to be involved in oral infectivity or formation of the entry complex (Wang et al., 2018). This finding seems not so surprising considering the fact that ac5 homologs are present only in a subset of the Alphabaculoviruses, in contrast to other pif genes. Whether AC108 is involved in either oral infectivity or complex formation still remains to be established.

\section{PIFs mediate viral entry under alkaline conditions in the midgut.}

Depending on the insect species, the $\mathrm{pH}$ in the larval midgut varies between 10 and 12 , which makes it probably the most alkaline environment in nature (Terra and Ferreira, 1994). Orally ingested OBs dissolve in this alkaline environment, releasing ODVs in the midgut lumen. The ODVs then first encounter the peritrophic membrane. This membrane is composed of chitin, mucopolysaccharides and proteins, and mechanically protects the epithelial midgut cells from 
the food bolus in the gut lumen (reviewed by Hegedus et al.,2009). Some baculoviruses encode metalloproteinases, called enhancins, which degrade the mucin component of the peritrophic membrane (Wang and Granados, 1997, Slavicek, 2012, Toprak et al., 2012). However, most baculoviruses, including AcMNPV, do not encode such enhancins, while their ODVs still pass the peritrophic membrane. Hence, it is unclear to which extent the peritrophic membrane is a barrier for ODVs to reach the midgut epithelial cells and whether PIFs are involved in passing this structure as recognized by Rohrmann et al., 2013. PIF8 (P95) is so far the only PIF for which experimental data support a role in assisting ODVs in passing the peritrophic membrane (see section PIF8 below).

Once ODVs have passed the peritrophic membrane, they infect midgut columnar cells via microvilli that form so-called brush borders at the luminal side of these cells. Electron microscopic analysis of the brush border of Trichuplusia ni larvae showed numerous nucleocapsids inside microvilli within four hours of inoculation (Kawanishi et al., 1972, Granados and Lawler, 1981). Furthermore, virus-binding assays revealed that ODVs bind to the brush borders in a saturable manner and that binding was significantly reduced upon protease pre-treatment of the brush borders (Horton and Burand, 1993). These findings suggest that the ODVs bind to an as yet unknown proteinaceous receptor on the microvilli. The subsequent fusion between the ODV-envelope and the microvillar cell membrane has been demonstrated by octadecyl rhodamine (R18) de-quenching assays (Fig. 6). In this assay,

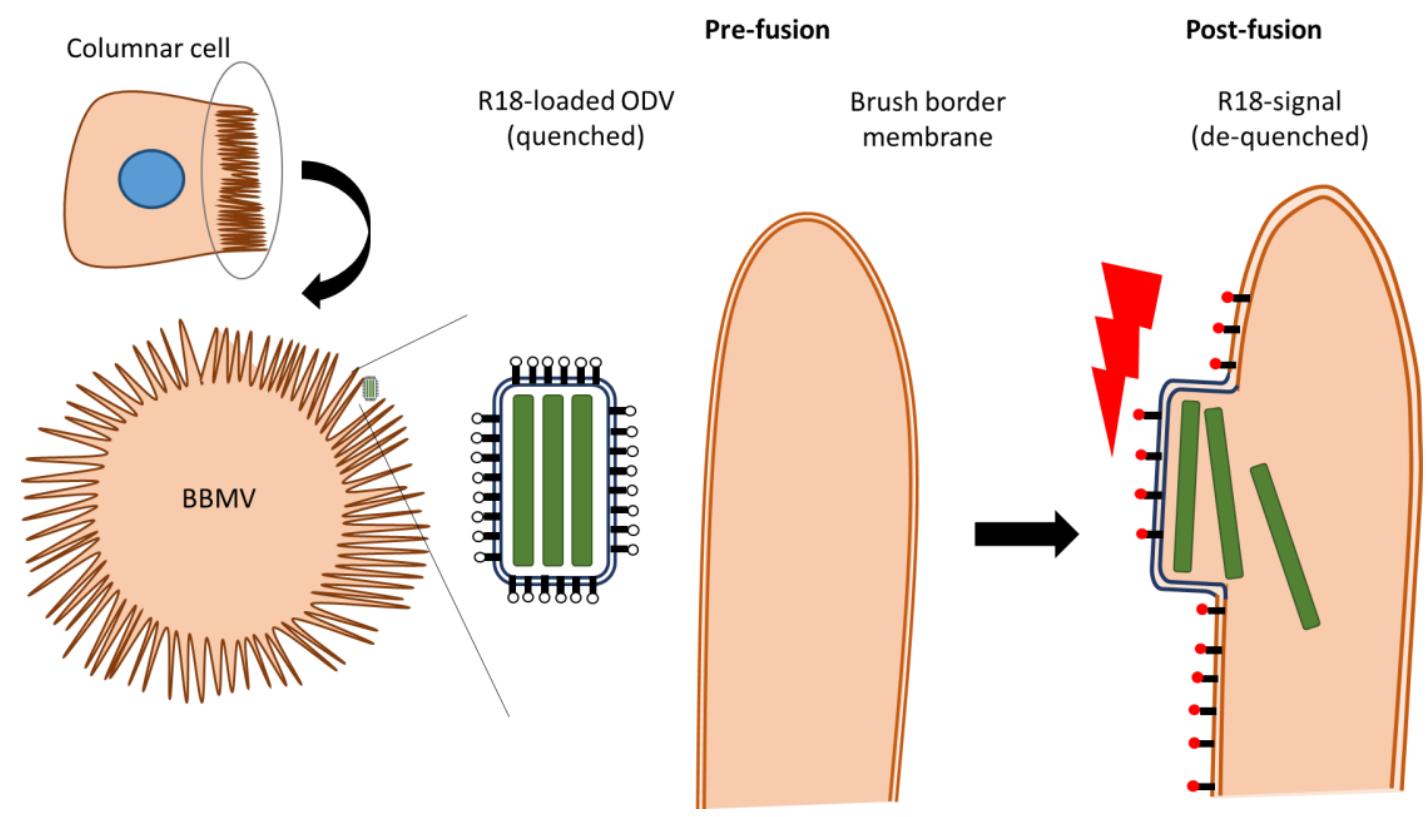

Figure 6: Schematic representation of the R18 de-quenching assay. The fluorescent activity of R18 is initially quenched in the ODV envelope because of the high concentration. Upon membrane fusion, R18 dilutes and gives a fluorescent signal. 
isolated brush border membrane vesicles (BBMV), an in vitro model for the columnar cell's brush border, were mixed with ODVs that were loaded with high concentrations of the fluorescent dye R18. Due to the high concentration of R18 in the ODV-envelope, the fluorescence of the dye is quenched. However, upon fusion of the envelope with the BBMV, $\mathrm{R} 18$ dilutes in the fused membranes which results in de-quenching of the fluorescent signal that then can be recorded (Hoekstra et al., 1984). This approach revealed that the fusion between ODVs and BBMV's occurs at 4 and $27^{\circ} \mathrm{C}$, and in a pH-range of 4-11 (Horton and Burand, 1993). Binding and fusion was most efficient at $27{ }^{\circ} \mathrm{C}$ under alkaline conditions. Therefore fusion via the endocytic pathway, as shown for BVs, is less likely as this pathway takes place under acidic conditions (Horton and Burand, 1993, Blissard and Wenz, 1992, Monsma and Blissard, 1995). Microvilli seem to be suitable structures for membrane fusion, as their cylindrical shape and narrow diameter facilitates interactions between membranes. The curvatures create disorder in the membrane, which may facilitate intermixing of (in this case viral and host) membranes during fusion (Wilson and Snell, 1998). In addition, the microvilli provide a scaffold for adhesion molecules in which polarized actin filaments create microdomains that can concentrate adhesion and fusion complexes at the tip of the microvilli (Wilson and Snell, 1998). On the side of the virus, PIFs play an essential role in virus entry into the midgut epithelial cells. These proteins are implicated in binding, membrane fusion and further downstream processes like intracellular transport of the nucleocapsids to the nucleus. In the following sections, PIF functions will be discussed.

\section{PIFs forming the stable core of the ODV entry complex}

\section{PIF1 and 2 mediate binding to the columnar cell microvilli.}

PIF1, 2, 3 and 4 form the stable core in the ODV entry complex. Functionally, PIF1 and PIF2 appear to be involved in binding of ODVs with the microvilli of columnar cells, as has been demonstrated by Ohkawa et al., 2005 with R18 de-quenching assays (the technique is explained above). These assays showed that absence of either PIF1 or PIF2 in the ODV envelope resulted in a threefold decrease in ODV binding compared to wild type. However, once these crippled ODVs had bound, at least half of them were able to fuse with the epithelial membrane, similar as for the wild type virus. This indicates that the absence of either PIF1 or 2 affects ODV binding but not fusion. Live imaging of ODVs with GFP-tagged nucleocapsids also showed that at least a fraction of the ODVs of pifl- and pif2 deletion mutants were still able to bind to the microvilli of freshly isolated epithelial cells (Mu et al., 2014). The finding that PIF1 and 
PIF2 are involved in ODV binding is in accordance with predictions of the Phobius algorithm, which indicate that the topology of these PIFs in non-cytoplasmic (Fig. 1). In this orientation, these PIFs point out from the ODV envelope after virion envelopment, according to the model of Shi et al., 2015, making them available for interaction with host cell receptors. However, as PIF1 and PIF2 are essential for formation of the stable core and therefore the ODV entry complex (Peng et al., 2010), the observed phenotypes in absence of these PIFs may (at least in part) result from lost interactions with other components of the entry complex. So whether PIF1 and PIF2 are involved in ODV-binding via direct interactions with host factors or indirect by facilitating formation of the entry complex or may be even both is not clear.

\section{PIF3 mediate oral infectivity after binding and fusion.}

PIF3, the third component that is essential for formation of the core complex (Peng et al., 2010), is not involved in ODV binding and fusion as was determined by R18 de-quenching assays (Ohkawa et al., 2005). It was proposed that PIF3 is involved in oral infectivity in a process downstream from binding and fusion, for example by aiding translocation of the nucleocapsids to the nucleus. This is supported by predictions of the Phobius algorithm, which indicate that PIF3 is cytoplasmic oriented in the host cell and thus is probably directed towards the nucleocapsids and would therefore not be available to interact with a host receptor (Fig. 1). Furthermore, Song et al., 2012 reported the presence of low levels of PIF3 in the nucleocapsid fraction of HearMNPV ODVs, suggesting an interaction with the nucleocapsid. The finding that pif3-deletion does not affect ODV binding and fusion, implies that binding and fusion may also occur in absence of the entry complex as its stable core cannot be formed in absence of PIF3 (Peng et al., 2010).

Predictions of the Phobius algorithm also indicate that PIF3 has an N-terminal transmembrane domain (Fig. 1). Analysis of viruses with PIF3 truncations- and amino acid substitutions revealed that the $\mathrm{N}$-terminal transmembrane domain is crucial for routing to the inner nuclear membrane, but that the final incorporation into the ODV-envelope is mediated by other parts of PIF3 (Li et al., 2007). Two PIF3 truncation mutants, in which either twenty or forty amino acids were deleted downstream from the $\mathrm{N}$-terminal transmembrane domain, were routed to the inner nuclear membrane, but were not incorporated into the ODV-envelope. The ODVs of these mutant viruses were not orally infectious and are as such phenotypically the same as a pif 3 deletion mutant. Similar results were found when the C-terminal forty amino acids were removed. Two mutants, however, a twenty amino acid C-terminal truncation mutant and a 
mutant in which the cysteine residue C164 was replaced by a glycine, still showed a low-level of oral infectivity while these mutated forms were also not detected in the ODV envelope by western blot analysis. Of all mutants, the $\mathrm{C} 162 \mathrm{G}$ substitution mutant is particularly interesting as the oral infectivity was severely affected, while this form of PIF3 was still incorporated into the ODV-envelope. The C162G mutant is the only PIF3 mutant where loss of oral infectivity can be directly related to the mutation itself, and not to side-effects as affected routing. This study demonstrates that it can be difficult to analyse PIF-proteins functions by mutagenesis as this might affect routing to the ODV envelope.

\section{The role of PIF 1-3 in host-range determination}

PIFs that mediate ODV-binding seem to be host-range determinants, as resistance of Spodoptera frugiperda larvae to AcMNPV could partially be explained by differences in efficiencies in ODV-binding to the midgut, when compared to ODVs of Spodoptera frugiperda multiple nucleopolyhedrovirus (SfMNPV) (Haas-Stapleton et al., 2005). This was further supported by an experiment, in which either PIF1 or 2 of Helicoverpa armigera nucleopolyhedrovirus (HearNPV) were replaced by their homologs from Spodoptera litura nucleopolyhedrovirus (SpltNPV). The resulting HearMNPV hybrids with the single pif-gene replacements were not orally infectious for Helicoverpa armigera larvae (Song et al., 2016). In contrast, a hybrid HearNPV, in which PIF3 was substituted for the SpltNPV homologue from, was able to infect $H$. armigera larvae. PIF3 is not involved in ODV binding and apparently, the biological function of PIF3 is (to a certain extend) inter exchangeable between these viruses (Ohkawa et al., 2005). On the other hand, PIF1 and 2 seemed to mediate ODV-binding species specifically. In these experiments, it has not been analysed whether the hybrid virus was able to form a complex. So it remains to be established whether the failing binding properties of these PIFs affected oral infectivity or whether these PIFs are not compatible with the remaining native PIFs to form a functional entry complex.

PIF4 provides proteolytic resistance to the stable core.

PIF4 was identified when an AcMNPV ac96 deletion mutant was not able to orally infect Trichuplusia ni larvae (Fang et al., 2009). Similar results were obtained with HearMNPV and Bombyx mori NPV (BmNPV) after deletion of the ac96 homologs ha85 and bm79 (Huang et al., 2012, Dong et al., 2014). In AcMNPV, PIF4 was found in the ODV and BV envelopes by western blot analysis (Fang et al., 2009), but this was not supported by mass spectrometry 
analysis of the virus particles (Braunagel et al., 2003, Liu et al., 2008, Wang et al., 2010). However, PIF4 was detected by mass spectrometry after immune precipitation of PIF1, indicating that the complexity of the protein samples affected detection of this protein when analysing whole virus particles (Peng et al., 2012). Nevertheless, the pif4 deletion mutant of AcMNPV showed wild type levels of BV production and mortality in injection assays (Fang et al., 2009). In ODVs of AcMNPV, PIF4 is essential for oral infectivity and is part of the core complex (Peng et al., 2012). However, unlike pif1, 2 and 3, deletion of the pif4-gene (ac96) did not affect interactions between the other components of the core complex as a smaller stable complex made-up of PIF1, 2 and 3 was found in the pif4 deletion mutant.

This smaller complex was only found in ODVs that were released from OBs obtained from cell culture infections (C-OBs) and not in ODVs from larval-derived OBs (L-OBs) (Chapter 3). When analysing ODVs from L-OBs, the smaller complex appeared to be degraded by hostderived proteases that were co-occluded in the OBs. When these proteases were inactivated by heat treatment of L-OBs prior to their dissolution in alkaline buffer and isolation of the ODVs, the smaller complex was found again, like in ODVs from C-OBs. Apparently, the presence of PIF4 in the stable core provides the core complex resistance against proteolytic degradation by alkaline proteases.

Such a property of PIF4 would also explain the, at the time contradictory, results obtained earlier with HearNPV, where no smaller complex with PIF1, 2 and 3 was found after deletion of the pif4-homolog ha85 (Huang et al., 2012). As these analyses were performed with larvalderived ODVs, the smaller complex could have been degraded by host-derived proteases. Another contradicting finding was that HA85 was not detected in the stable core by western blot and that immunoprecipitation of HearMNPV PIF1 resulted in co-precipitation of PIF0, PIF2 and PIF3, but not HA85 (Huang et al., 2012). To which extend the experimental conditions or the presence of host-derived proteases complicated these analyses is not clear. A yeast-two-hybrid assay nevertheless identified interactions between PIF4-PIF0, PIF4-PIF1, PIF4-PIF2 and PIF4-PIF3 (Huang et al., 2012). Similar interactions of PIF4 with PIF1, PIF2 and PIF3 were also identified in BmNPV by bimolecular fluorescence protein complementation assays (BIFC) and co-immunoprecipitation studies after overexpression of these PIFs (Dong et al., 2014).

In conclusion, there is strong evidence that in addition to AcMNPV, other baculovirus species form a core complex with PIF1, PIF2, PIF3 and possibly also PIF4. However, the exact nature 
of the protein-protein interactions needs further validation under native conditions. Although PIF4 in AcMNPV appears not to be important for interactions between the remaining components of the stable core (PIF1-3), this PIF is required for the formation of the entire ODV entry complex as this complex was not detected in the ODV-envelope, released from C-OBs, of the pif4 deletion mutant with blue native-PAGE (Peng et al., 2012). These results suggest that PIF4 is important for establishing interactions between the stable core and the other associated PIFs and that these interactions are crucial for oral infectivity. The pif4 deletion mutant has not been tested so-far in R18 de-quenching or live imaging assays.

\section{PIFs that associate with the core complex}

\section{PIFO mediates ODV-binding with the midgut epithelium.}

Historically, P74 was the first ODV-envelope protein that was found to be essential for oral infectivity and is now also named PIF0 (Kuzio et al., 1989). This PIF-protein lacks an Nterminal signal sequence for routing to the inner nuclear membrane. Instead, PIF0 has two Cterminal transmembrane domains, which are essential for nuclear translocation of this protein (Fig. 3) (Slack et al., 2001, 2010, Yao et al., 2004). The Phobius-algorithm predicts that PIF0 is C-terminally anchored to the ODV envelope and that the N-terminal part is directed outwards from the ODV-envelope, because of its non-cytoplasmic orientation in the host cell. Such a topology is in accordance with results from R18 de-quenching assays and live imaging of fluorescent ODVs that showed that PIF0 is important for ODV binding with midgut epithelial cells (Haas-Stapleton et al., 2004, Mu et al., 2014). When ODVs lack PIF0, one third of these bind to the midgut epithelium, compared to wild type ODVs (Haas-Stapleton et al., 2004). Once bound, about half of the bound ODVs fused with the epithelial membrane, just like wild type ODVs. This suggests that fusion is not affected by the absence of PIF0, as reasoned by HaasStapleton et al., 2004. Two independent studies showed that incorporation of PIF0 in the ODVenvelope seems not absolutely required to be able to play a role in oral infectivity (Yao et al., 2004, Slack et al., 2010). The first study showed that co-feeding of purified PIF0-GFP fusion protein along with ODVs of pifo deletion mutant, rescued oral infectivity (Yao et al., 2004). Similar results were obtained with purified C-terminal truncated variants of PIF0, lacking the transmembrane domains. The co-feeding of these soluble forms of PIF0 also rescued the oral infectivity of a pifO deletion mutant (Slack et al., 2010). Apparently, the C-terminal transmembrane domains of PIF0 are dispensable for oral infectivity, while the rest of the protein mediates oral infectivity via interactions with other PIFs and likely also via interactions with 
host factors. Co-immunoprecipitation studies demonstrated that PIF0 interacts with PIF1 and PIF3 and further analysis revealed that PIF0 was a component of the entry complex, which was observed only under non-denaturing conditions (Peng et al., 2010, 2012). Apparently, PIF0 associates with the core with relatively low affinity and might therefore be regarded as a more loosely associated component of the ODV entry complex than PIF1-4.

PIFO is cleaved by co-occluded proteases and trypsins.

PIF0 is proteolytically cleaved in two separate events, once by a host-derived protease upon OB dissolution in the alkaline environment of the midgut and once by trypsins, present in the gut of the larva (Fig. 7). In the first event, PIF0 is cleaved into two fragments of approximately 35 and $40 \mathrm{kDa}$ (Peng et al., 2011). This cleavage was observed with ODVs released from LOBs, but not with ODVs from C-OBs, indicating that the protease was obtained from the larval host. After cleavage, both fragments remain associated with the core complex as these precipitated together with PIF1 in a co-immunoprecipitation study with PIF1 antiserum (Peng et al., 2011). When the ODV entry complex lacks one of the components of the core complex, the cleaved form of PIF0 was not found, but instead PIF0 was degraded. This suggests that the conformation of the ODV entry complex prevents degradation of PIF0, resulting in cleavage of this PIF (Chapter 3). However, the importance of this cleavage event for oral infectivity of ODVs is not clear as a bioassay with $T$. $n i$ larvae showed that L-OBs and C-OBs resulted in a

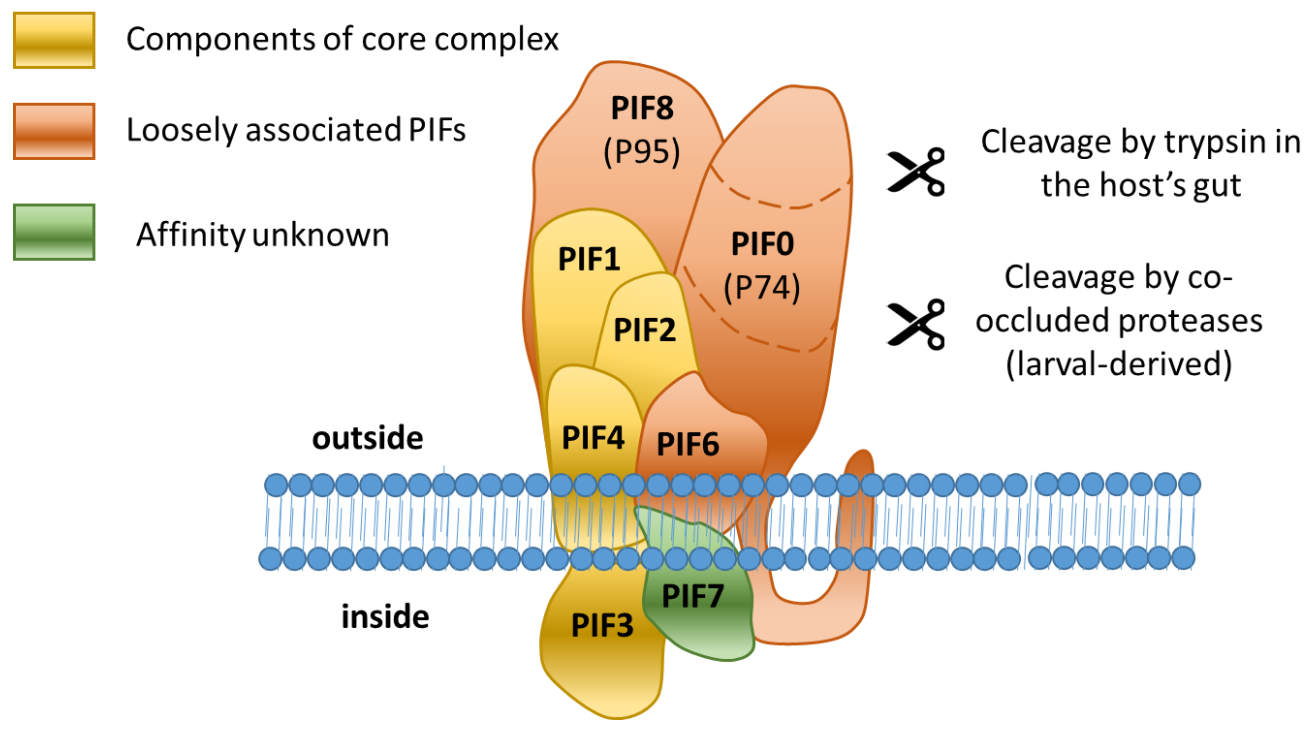

Figure 7: Two step cleavage model of PIF0. PIF0 is first cleaved by co-occluded host-derived proteases when larval-derived OBs dissolves in an alkaline environment. These 35 and $40 \mathrm{kDa}$ fragments remain associated with the core complex. Secondly, a $20 \mathrm{kDa}$ N-terminal fragment is cleaved off by trypsin in the host's gut. 
similar level of mortality, although the L-OBs killed these larvae slightly faster than C-OBs (Bonning et al., 1995).

In the second cleavage event, a $20 \mathrm{kDa}$ fragment is cleaved from the $\mathrm{N}$-terminus of PIF0 by trypsins present in the midgut. This cleavage event was observed when purified GFP-tagged PIF0 was incubated with BBMV's made from endothelial cells of third instar Helicoverpa zea or fourth instar T. $n i$ larvae (Slack et al., 2005, 2008). This trypsin-mediated cleavage appears important for oral infectivity as inhibition of cleavage by soybean trypsin inhibitor or through mutation of conserved arginine residues in the cleavage site affected the infectivity of ODVs (Slack et al., 2008). However, the trypsin inhibitor might inhibit, in addition to the cleavage of PIF0, yet unidentified cleavage events of other ODV-envelope proteins that might also affect oral infectivity of ODVs. Furthermore, the effects of a mutated cleavage site on oral infectivity was determined by feeding isolated OBs to T. $n i$ larvae, while the presence of the modified PIF0molecules was only validated in transfected insect cells and not in the ODVs. So when these mutations would affect proper routing of PIF0, as shown before with some PIF3 mutants, the decreased oral infectivity might be explained by absence of (mutated) PIF0 in the ODV envelope. However, trypsin-mediated cleavage of PIF0 was also observed in wild type ODVs, where this PIF associates with other PIFs in the entry complex. This indicates that this cleavage event also occurs under native conditions and might therefore be important for oral infectivity of ODVs (Slack et al., 2008).

PIF6 is also a component of the ODV entry complex.

The ODV-envelope protein AC68 was identified as PIF6 (Nie et al., 2012), when a lef3-ac68 double deletion bacmid was repaired with lef3 and the resulting ac68 deletion virus displayed the phenotype of the typical pif deletion mutant. PIF6 was not only found in the envelope of ODVs, but also in BVs (Nie et al., 2012). Its function in BVs is unknown, since BV production and infectivity were unaffected in absence of this protein. In ODVs, PIF6 precipitated together with PIF1 in a co-immunoprecipitation study with PIF1 antiserum and was identified as part of the entry complex under non-denaturing conditions (Peng et al., 2012, Chapter 3). So PIF6 appears to be important for ODV oral infectivity in the context of the entry complex. Predictions with the Phobius algorithm revealed this PIF does not have an INM-SM, but instead has a Cterminal hydrophobic domain and a non-cytoplasmic oriented N-terminus (Fig. 3). This indicates that PIF6 is probably directed outwards from the ODV envelope. However, the 
biological importance of PIF6 for ODV binding, fusion or for a downstream process as hypothesized for PIF3, has yet to be determined.

The zinc-finger domain of PIF8 is important for oral infectivity.

AC83 (also known as VP91 and P95) has two distinct roles during the infection cycle. Firstly, AC83 is required for nucleocapsid formation and is therefore essential for the production of BVs and ODVs (Zhu et al., 2013). Secondly, AC83 is involved in oral infectivity. The protein has a zinc-finger (ZF) domain (also addressed as chitin-binding domain (CBD)) and mutants that lack this domain show the phenotype of a pif deletion mutant. Although this protein has multiple roles in the infection cycle, it is considered to be a PIF and is now named PIF8. Oral infectivity of ODVs of such a mutant was partially rescued by feeding OBs to larvae together with calcofluor white, an agent known to damage the peritrophic membrane (Zhu et al., 2013). A similar effect was not noted for viruses with a deletion of $p 74$, pif1, 2 or 3 (Song et al., 2008). These data suggest that PIF8 binds to the chitin component of the peritrophic membrane. Affinity for chitin might also be advantageous for binding to the brush borders of epithelial cells as brush border microvilli produce chitin for the peritrophic membrane (Rohrmann, 2013). Possible interaction of PIF8 with host factors is also supported by the Phobius algorithm, which indicates that the topology of this protein is non-cytoplasmic, so is directed outwards from the ODV envelope (Fig. 1).

In the ODV envelope, PIF8 associates with the ODV entry complex with lower affinity than the components of the stable core, as this PIF was only found in the complex under nondenaturing conditions (Peng et al., 2012). As this complex was not found in the mutant virus with the truncated form of PIF8, that lacks the ZF-domain, it was hypothesized that this domain is involved in formation of the entry complex by recruiting other PIFs (Javed et al., 2017). However, this hypothesis was not experimentally supported as it was not determined whether the other PIFs (and the truncated PIF8 itself) were indeed absent in the envelope of mutant ODVs.

\section{PIF5 might operate independent from the entry complex.}

ODV-E56 (AC148) has been established as PIF5 on the basis that deletion of the corresponding gene significantly impaired oral infectivity of ODVs (Harrison et al., 2010, Sparks et al., 2011a). However, low-level mortality has consistently been observed after deletion of this gene, suggesting that PIF5 is important but not crucial for oral infectivity, in contrast to the other 
PIFs. PIF5 has a C-terminal hydrophobic domain for translocation to the ODV envelope (Fig. 3) (Braunagel et al., 1996). When this domain was substituted for $\beta$-galactosidase, PIF5 translocated to the virogenic stroma and finally ended up in the nucleocapsids, instead of in the ODV envelope (Braunagel et al., 1996). PIF5 was not detected in the entry complex and did not co-immune precipitate with PIF1 (Peng et al., 2012). However, in a yeast-two-hybrid analysis PIF5 reciprocally interacted with PIF3, a component of the core complex (Peng et al., $2010 \mathrm{~b}$ ). Whether this interaction also occurs in the ODV envelope and in the presence of all other PIFs has yet to be determined. Like PIF3, PIF5 seems also not to be involved in ODV binding and fusion (Sparks et al., 2011a). It could therefore be speculated that PIF5 mediates oral infectivity after binding and fusion, may be in association with PIF3. At this moment, the post-fusion configuration of PIF-proteins is entirely enigmatic though. It is presently also unknown whether the entry complex remains intact, falls apart, or even changes its composition after ODV binding and fusion.

\section{ODV-E66 can retrospectively also be assigned as a PIF.}

ODV-E66 (ac46) is important but not crucial for midgut infection as deletion of this gene, like with PIF5, only impairs oral infectivity of ODVs (Sparks et al., 2011b, Xiang et al., 2011). This protein mediates oral infectivity possibly by (co-) facilitating ODV-binding with the midgut epithelium as peptide-derivatives of ODV-E66 compete with ODVs for binding with BBMVs (Sparks et al., 2011b). These results are in accordance with predictions of Phobius-software

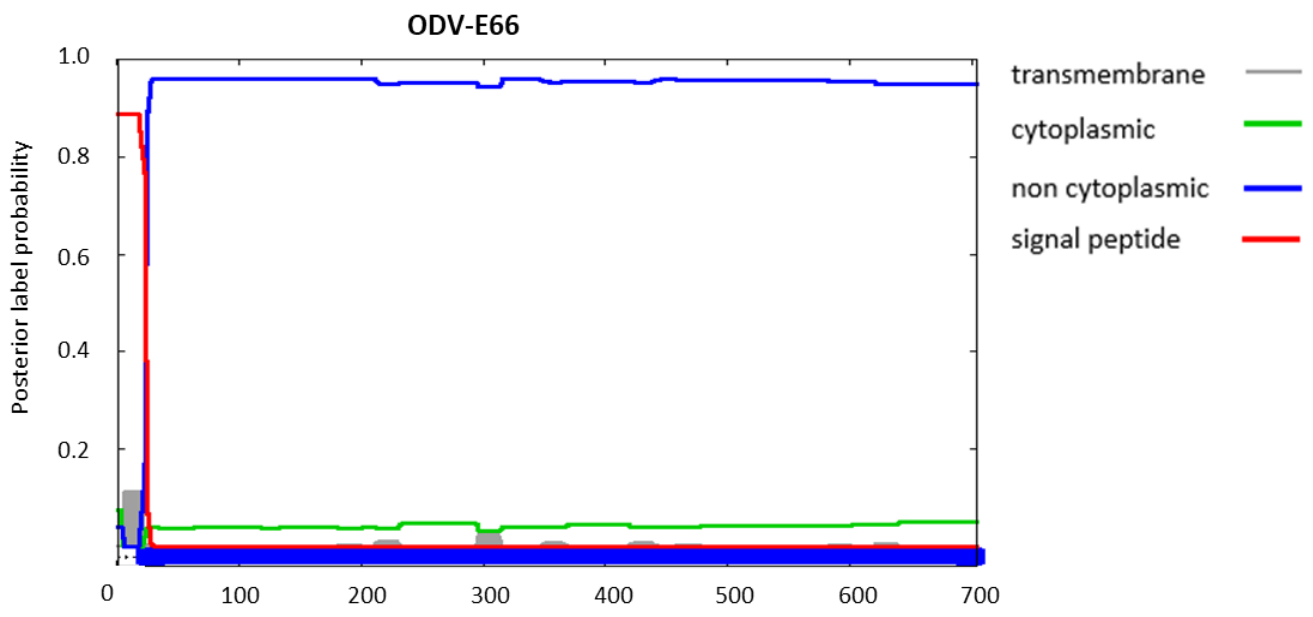

Figure 8: Prediction the presence of a transmembrane domain and signal peptides in ODV-E66 and orientation of this protein in an infected host-cell with the Phobius algorithm (Käll et al., 2004). On the yaxis: the probability for the presence of transmembrane domains (grey), cytoplasmic (green) or non-cytoplasmic (blue) orientation of the sequence and a signal peptide (red). The final result of the prediction is summarized by the bar at the bottom with the indicated colours. The $\mathrm{x}$-axis shows the number of the amino acids of that specific sequence. 
that indicates that ODV-E66 is N-terminally anchored in the ODV envelope by its INM-SM and that the rest of the protein is directed outwards from the ODV envelope, because of its noncytoplasmic topology in the host cell, enabling interactions with host factors (Fig. 8). Two studies indicated that ODV-E66 interacts with other components of the ODV entry complex. In a yeast-two-hybrid analysis, ODV-E66 reciprocally interacted with PIF2 and PIF3 (Peng et al., 2010b). Furthermore, in co-immunoprecipitation studies and bimolecular fluorescence protein complementation assays with BmNPV, ODV-E66 interacted with the PIF4 homolog BM79 (Dong et al., 2014). In light of this, it is interesting to determine whether ODV-E66 associates with the ODV entry complex. As ODV-E66 appears to be important (but not crucial) for oral infectivity by (co-) facilitating ODV-binding with the midgut epithelium, the protein can retrospectively be assigned as a PIF.

\section{Perspectives and conclusions}

The infection cycle of baculoviruses starts with infection of midgut epithelial cells. To mediate this infection, the ODV envelope contains an array of at least nine different PIFs and possibly also ODV-E66. The genes that encode these proteins are highly conserved among all baculoviruses and, with exception of ac110 (encoding PIF7), in other large, nuclear replicating invertebrate DNA viruses (Reviewed by Wang et al., 2017). The fact that the pif-genes are found in a large group of evolutionary related invertebrate DNA viruses that have co-evolved with their hosts for millions of years, suggest that PIF-mediated entry is a commonly used and ancient entry mechanism (Thézé et al., 2011). The number of PIFs seems to increase gradually during evolution, from PIF0-PIF3 in the Nimaviridae to all nine in the Baculoviridae, suggesting that PIF-mediated entry became more and more advanced over time (Wang et al., 2017). As said, ten different proteins are known to be involved in midgut infection by baculovirus ODVs, but there are maybe even more. A good candidate is AC108 that precipitated together with PIF1 (Peng et al., 2012).

Furthermore, eight out of nine PIFs form a large complex in the ODV envelope, suggesting that PIFs cooperatively mediate cell entry in a network of protein-protein interactions. This finding complicates the interpretation of studies with pif-deletion mutants as the observed phenotypes concerning ODV binding and fusion cannot be directly attributed to absence of a PIF-protein itself, but may be a consequence of indirect effects because of lost protein-protein interactions. Hence, making deletion mutants is a good approach to identify PIFs, but not to elucidate the molecular mechanism of PIF-mediated cell entry or to identify the biological importance of a 
single PIF in that process. Now that we know that there are many PIF-proteins of which at least eight form a complex, the questions that emerge are: what is the role of the ODV entry complex during midgut infection and does the various PIFs have different functions?

In absence of PIF3, a crucial protein for complex formation, ODVs are still able to bind and fuse with the plasma membrane of columnar cell brush borders, but nevertheless fail to infect the larval midgut. This suggests that the entry complex itself is not crucial for binding and fusion, but probably mediates infection by serving as a scaffold for proteins that mediate binding and fusion (PIF0, 12 and maybe also ODV-E66), and proteins involved in downstream processes (Such as PIF3 and PIF5). However, what these downstream processes are and what other function(s) PIFs have besides mediating binding and fusion during midgut infection is not clear.

Normally, the midgut epithelium digests and absorbs nutrients. Viruses may exploit this physiology for entry and passage of the midgut barrier. For example, the Jujonia coenia densovirus is transported across the midgut epithelium of $S$. frugiperda larvae via transcytosis (Wang et al., 2013), which is normally used to transport large unprocessed proteins (Casartelli et al., 2005,). Furthermore, infection with this densovirus altered the structural and functional properties of the epithelial tissue is such a way that it negatively affects its gate function. However, how infection with baculoviruses affects the midgut physiology and how PIFs are involved in this is unexplored. These insights might be important to define what other processes besides ODV binding and fusion are required for successful infection of the midgut epithelium.

\section{Acknowledgements}

This work was supported by grant 824.14 .16 of the Netherlands Organization for Scientific Research (NWO). 
$-28-$ 


\title{
Chapter 3
}

\section{Baculoviruses require an intact ODV entry complex to resist proteolytic degradation of per os infectivity factors by co-occluded proteases from the larval host}

Bob Boogaard, Jan W. M. van Lent, David A. Theilmann, Martin A. Erlandson, Monique M. van Oers

\begin{abstract}
Baculoviruses orally infect caterpillars in the form of occlusion-derived viruses (ODVs). The ODV-envelope contains a number of proteins which are essential for oral infectivity, called per os infectivity factors (PIFs). Most of these PIFs are involved in the formation of an ODV-entry complex that consists of a stable core, formed by PIF1, PIF2, PIF3 and PIF4, and the more loosely associated PIFs PIF0, 7 and 8. PIF6 was in this study also identified as a loosely associated component of the entry complex. PIF1, PIF2 and PIF3 are essential for formation of the stable core, whereas deletion of the pif 4 gene results in the formation of a smaller complex. PIF0 is not needed for formation of the stable core. We show here in larva-derived ODVs of the Autographa californica multicapsid nucleopolyhedrovirus that PIF-proteins are degraded by host-derived proteases after deletion of a single pif-gene. Constituents of the stable corecomplex appeared to be more resistant to proteases as part of the complex than as monomer, as in ODVs of a pifO deletion mutant only the stable core was found but no PIF monomers. When the stable core lacks PIF4, it lost its proteolytic resistance as the resulting smaller core complex was degraded in a pif4 deletion mutant. The core was also sensitized to proteolytic degradation in absence of PIF6. We conclude from these results that an intact entry-complex in the ODVenvelope is prerequisite for proteolytic resistance of PIF-proteins under the alkaline conditions of the larval midgut.
\end{abstract}

This chapter has been published as Boogaard, B., Van Lent, J. W. M., Theilmann, D. A., Erlandson, M. A., \& van Oers, M. M. (2017). Baculoviruses require an intact ODV entry-complex to resist proteolytic degradation of per os infectivity factors by co-occluded proteases from the larval host. Journal of General Virology, 98, 3101-3110. 


\section{Introduction}

Baculoviruses infect the larval stages of insects in the orders Lepidoptera, Hymenoptera and Diptera (Jehle et al., 2006). The name baculovirus refers to the bacillus-shaped nucleocapsid, in which a large circular, double-stranded DNA genome is packaged. The genome sizes range from 80-180 kbp, encoding 90-180 genes (Williams et al., 2017). Baculoviruses have a biphasic replication cycle in which two virion-types are produced, first the budded virus particles (BVs) and later in infection occlusion-derived virus particles (ODVs). BVs consist of a single nucleocapsid which is enveloped by a membrane derived from the plasma membrane of the host cell. BVs establish systemic infection within the larvae after a primary infection. ODVs are of two types, single- or multiple nucleocapsids in single capsid- (SNPVs) and multicapsid nucleopolyhedroviruses (MNPVs), within an envelope derived from the inner nuclear membrane of the host cell. ODVs are occluded in a crystalline protein-matrix of polyhedrin, forming occlusion bodies (OBs). When larvae consume OB-contaminated plant material, ODVs establish the primary infection in the insect. The OBs dissolve in the alkaline environment of the insect midgut to release the ODVs. The released ODVs then enter insect midgut epithelial cells by means of the activity of per os infectivity factors (PIF-proteins) which are present in the ODV-envelope. So far, nine different PIF-proteins have been identified: P74 or PIF0 (encoded by the Autographa californica (Ac) MNPV gene ac138) (Faulkner et al., 1997), PIF1 (ac119) (Kikhno et al., 2002), PIF2 (ac22) (Pijlman et al., 2003), PIF3 (ac115) (Ohkawa et al., 2005), PIF4 (ac96) (Fang et al., 2009), PIF5 (ODV-E56/ac148) (Harrison et al., 2010), PIF6 (ac68) (Nie et al., 2012), PIF7 (ac110) (Liu et al., 2016) and PIF8 (ac83) (Zhu et al., 2013). Most of these PIFs have been shown to be part of an entry complex in the ODVenvelope that consists of a stable core complex and some more loosely associated components. The stable core complex is formed by PIF1, PIF2, PIF3 and PIF4 and resists treatment with Laemmli buffer containing $2 \%$ SDS and 5\% 2-mercaptoethanol at $50^{\circ} \mathrm{C}$ for SDS-PAGE analysis (Peng et al., 2010). Of these components, PIF1, PIF2 and PIF3 appeared essential for formation of the stable core, while deletion of pif4 resulted in the formation of a smaller complex of only PIF1, PIF2 and PIF3 (Peng et al., 2012). PIF0, 7 and 8 have been shown to associate with the core-complex in a more loose way, as these components can only be detected as part of the entry complex under non-denaturing conditions (Peng et al., 2012, Chapter 2). PIF0 appeared not essential to form the stable core complex as this complex can be formed by a pifO deletion mutant (Peng et al., 2010, 2012). PIF5 has not been found in association with 
the entry complex (Peng et al., 2012). Whether the other identified PIFs are part of the entry complex is currently not known.

Earlier studies reported proteolytic activity from co-occluded alkaline proteases in larvaderived OBs (L-OBs) for various baculovirus-host combinations (Eppstein and Thoma, 1975, Summers and Smith, 1975, Tweeten et al., 1978, Payne and Kalmakoff, 1978, Wood, 1980). The biological significance of this proteolytic activity is poorly understood, but it has been suggested that it may be important for optimal release of ODVs from the protein matrix of occlusion bodies, as significant amounts of the matrix protein polyhedrin was still associated with the ODVs after OB dissolution in alkaline buffer when the these proteases were inhibited (Wood, 1980). Although degradation of the matrix protein was most prominent, peptide analysis showed that proteins of the ODVs were also degraded during treatment with alkaline buffer (Wood, 1980). Furthermore, it has been shown that PIF0 is cleaved into two fragments by an endogenous co-occluded protease when ODVs were released from OBs that were produced in Spodoptera exigua larvae (Peng et al., 2011). PIF0 has not been found to be cleaved when the ODVs were isolated from cell culture-derived OBs (C-OBs), suggesting that the cooccluded protease was obtained from the larval host (Peng et al., 2011). As well, cleavage of PIF0 was not observed when the L-OBs were heated prior to the release of the ODVs as a consequence of heat-inactivation of these proteases. These data suggest that the presence of cooccluded proteases in larva-derived OBs have significant effects on PIF-proteins as shown with PIF0.

Here, we study how co-occluded proteases affect PIF-proteins by comparing isolated cellderived and larva-derived ODVs (C-ODVs and L-ODVs respectively) in SDS-PAGE analysis. By using wildtype and various pif deletion mutants for this analysis, we were able to compare the proteolytic sensitivity of PIF-proteins as part of the stable core-complex with monomeric PIF-proteins. This study revealed that deletion of only a single pif-gene resulted in enhanced proteolytic degradation of the remaining PIFs, regardless of whether the missing PIF is member of the stable core or a loosely associated component of the entry complex. So the entire entry complex, comprised of the stable core complex and loosely associated PIFs, needs to be intact to resist the proteolytic activity of co-occluded proteases from the larval host. 


\section{Results}

The PIF-core complex is affected by co-occluded proteases from the host.

Peng et al., 2010 showed that the PIF-core-complex appeared as a $170 \mathrm{kDa}$ band in western blot analysis with PIF1 antiserum, when a protein sample of isolated C-ODVs (released from cell-culture derived $\mathrm{OBs}$ ) was heated at $50^{\circ} \mathrm{C}$, instead of the $95^{\circ} \mathrm{C}$ normally used for complete denaturation. On the other hand, the complex completely dissociates after denaturation at $95^{\circ} \mathrm{C}$. Here, we compared the stability of the core-complex in presence and absence of lepidopteran proteases. Therefore, we analysed the complex of wildtype AcMNPV in C-ODVs and L-ODVs (released from OBs produced by cultured insect cells (C) or S. exigua larvae (L)) after heating at 50,60, 75 and $95^{\circ} \mathrm{C}$ by western blot analysis with PIF1 antiserum (Fig. 1). In C-ODVs, the $170 \mathrm{kDa}$ core-complex and the PIF1 monomers were found after heating at 50,60 and $75^{\circ} \mathrm{C}$, while only PIF1 monomers were found when the sample was heated at $95^{\circ} \mathrm{C}$ (Fig. 1). These results indicate that the complex is very stable and only dissociates after heating at temperatures above $75^{\circ} \mathrm{C}$. However, with L-ODVs, degradation products of the core complex between 120 and $70 \mathrm{kDa}$ were detected when the sample was heated at $50^{\circ} \mathrm{C}$ and monomers were not found, probably because of the proteolytic activity of co-occluded proteases present in L-OBs that are activated during ODV-release in alkaline solution (Fig. 1). When the protein sample was heated at 60 or $75^{\circ} \mathrm{C}$, the $170 \mathrm{kDa}$ complex was found again, as were the PIF1 monomers. Possibly,

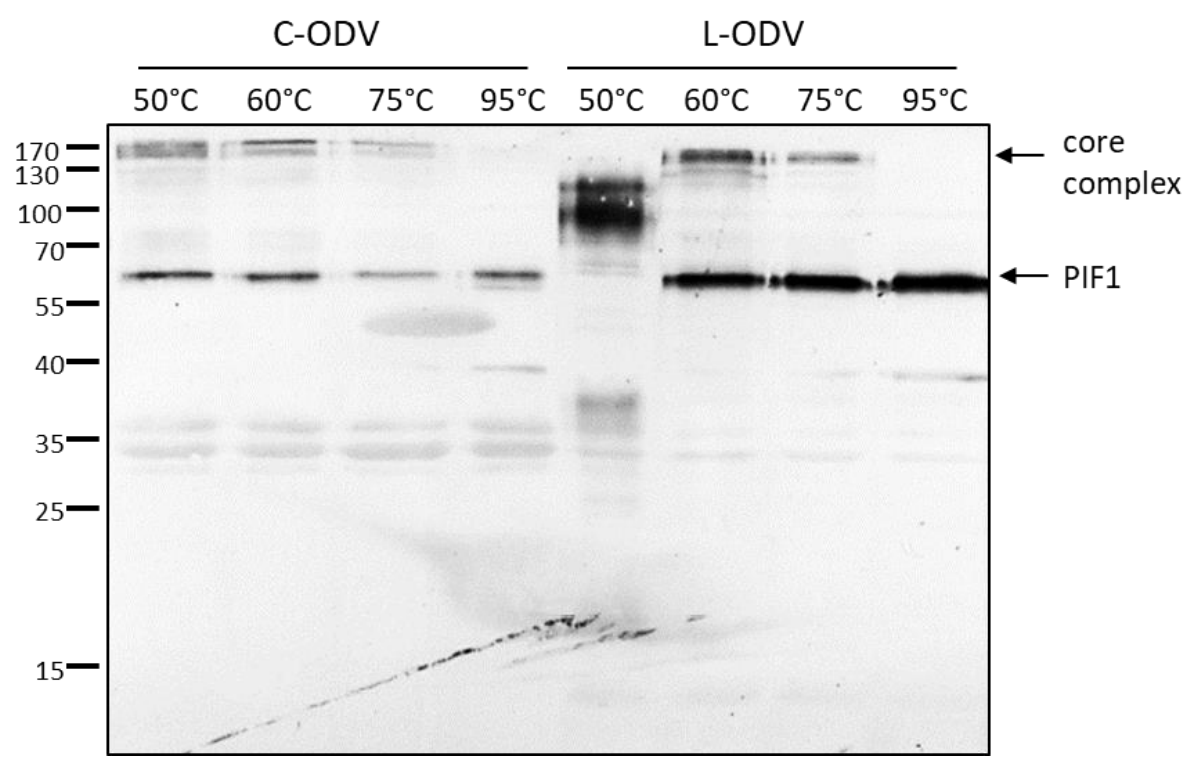

Figure 1: Temperature stability of the PIF core-complex and PIF1 monomers for wildtype AcMNPV, derived from cultured insect cells $(\mathbf{C})$ and infected larvae $(\mathbf{L})$. Wildtype ODVs were released from C-OBs (designated as C-ODV) and L-OBs (designated as L-ODV). The ODVs were purified and heated in Laemmli buffer at $50^{\circ} \mathrm{C}, 60^{\circ} \mathrm{C}, 75^{\circ} \mathrm{C}$ and $95^{\circ} \mathrm{C}$ for $5 \mathrm{~min}$ and separated by SDS-PAGE. Western blot analysis was performed with anti-PIF1 antiserum to detect the $170 \mathrm{kDa}$ core-complex and $60 \mathrm{kDa}$ PIF1 monomers. 
the higher incubation temperatures sufficiently inactivated the proteases in the L-ODV protein sample. As the complex of C-ODVs and L-ODVs were more comparable when heated during sample preparation at temperatures above $50^{\circ} \mathrm{C}$, all further analyses were conducted at 60,75 and $95^{\circ} \mathrm{C}$.

PIF1 is more resistant to proteolytic degradation as part of the core-complex than as monomer.

To assess how proteases affect PIF-proteins during OB-dissolution in a pif-deletion mutant that still contains the $170 \mathrm{kDa}$ core-complex in the ODV-envelop, C-ODVs and L-ODVs of a pifO deletion mutant were subjected to western blot analysis with PIF1 antiserum (Fig. 2). In CODVs, the complex and PIF1 monomers were found when heated at $60^{\circ} \mathrm{C}$ and $75^{\circ} \mathrm{C}$, and the complex was fully dissociated into monomers when completely denatured at $95^{\circ} \mathrm{C}$ (Fig. 2a), just as observed with the wildtype virus. In contrast, in L-ODVs of a pifO deletion mutant, only the core-complex was found when heated at 60 and $75^{\circ} \mathrm{C}$ but not the PIF1 monomers (Fig. 2b). Even after complete dissociation of the core-complex at $95^{\circ} \mathrm{C}$, no PIF1 monomers were found. As it was suspected that the co-occluded proteases degraded the PIF1 monomers, the proteases were inactivated by heat-treatment of L-OBs at $80^{\circ} \mathrm{C}$ for 40 minutes prior to ODV release in alkaline buffer. The PIF1 monomers were indeed detected again after heat treatment of L-OBs,

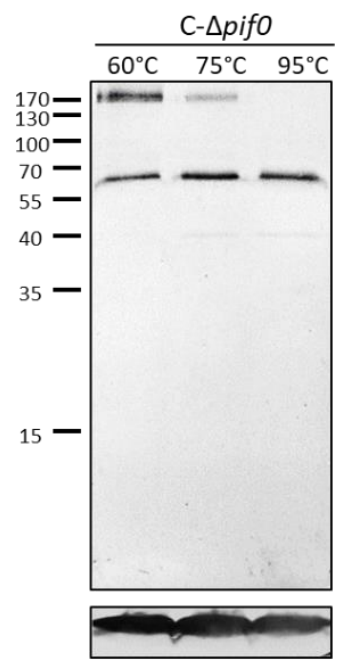

(a)

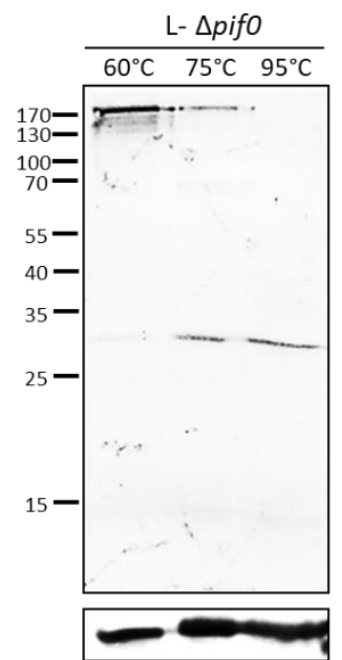

(b)

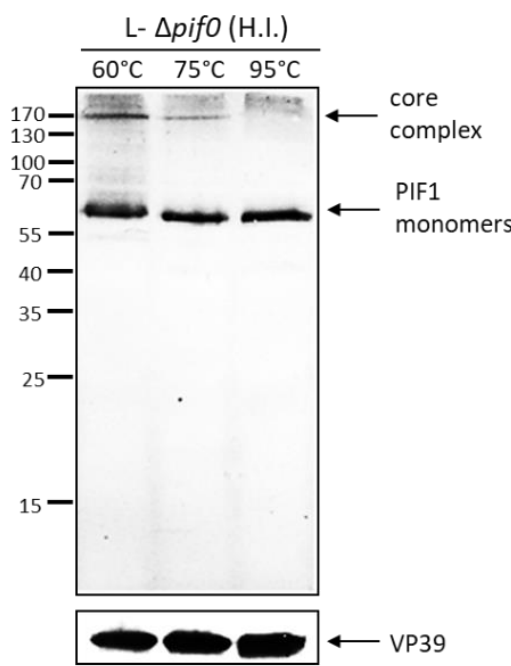

(c)

Figure 2: Western blot analysis of the PIF core-complex and PIF1 monomers in ODVs of the pif0 deletion mutant, derived from cultured insect cells (C) and infected larvae (L). ODVs of the pifO deletion mutant ( $\triangle p i f 0$ ) were released from C-OBs (a), L-OBs (b) or heat-inactivated L-OBs (c). For heat-inactivation (H.I.), the L-OBs were heated at $80^{\circ} \mathrm{C}$ for $40 \mathrm{~min}$ to inactivate co-occluded proteases, prior to dissolution in alkaline DAS-buffer. After purification of the ODVs, the samples were heated in Laemmli buffer at $60^{\circ} \mathrm{C}, 75^{\circ} \mathrm{C}$ or $95^{\circ} \mathrm{C}$ for $5 \mathrm{~min}$ and separated by SDS-PAGE. Western blot analysis was performed with antiserum against PIF1. Detection of capsid protein VP39 with VP39 antiserum was used as loading control (lower panels). 
further supporting the involvement of proteases in the degradation of PIF1 (Fig. 2c). After deletion of pifO, PIF1 is apparently more sensitive in monomeric form to the co-occluded proteases than as part of the stable core complex, which is still formed in this deletion mutant.

\section{PIF4 is important for the proteolytic resistance of the stable core-complex.}

Peng et al., 2012 showed that deletion of pif4 resulted in the formation of a smaller corecomplex of approximately $150 \mathrm{kDa}$, instead of the $170 \mathrm{kDa}$ core-complex of the wildtype virus. To determine the impact of the co-occluded proteases on this smaller complex, C-ODVs and L-ODVs of a pif4 deletion mutant were analysed by western blot analysis with antiserum against PIF1. When C-ODVs of a pif4 deletion mutant were analysed, the approximately 150 $\mathrm{kDa}$ complex and PIF1 monomers were detected with expected sizes when heated at $60^{\circ} \mathrm{C}$ (Fig. 3a). However, in L-ODVs, neither the smaller sized complex nor the PIF1 monomers were found after heating at $60^{\circ} \mathrm{C}$ (Fig. 3b, left panel). Only when the proteases associated with L-OBs were heat inactivated prior to L-OB dissolution in DAS-buffer, the $\sim 150 \mathrm{kDa}$ complex and PIF1 monomers were detected (Fig. 3b, right panel). Furthermore, when C-ODVs of a pif4 deletion mutant were analysed after heating at $75^{\circ} \mathrm{C}$, only PIF1 monomers were found and not the $\sim 150 \mathrm{kDa}$ complex. This was also observed in L-ODVs isolated from heat treated OBs. Thus the smaller complex that lacks PIF4 completely dissociates into monomers when heated

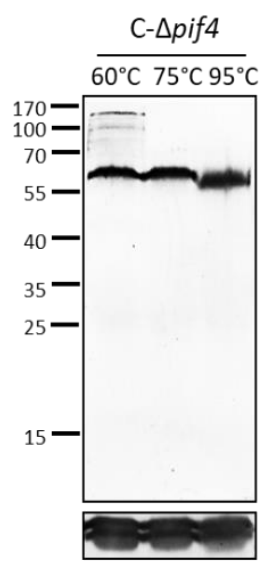

(a)

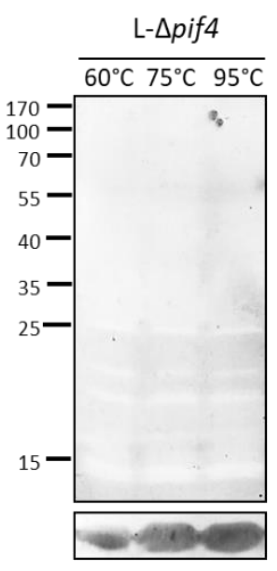

(b)

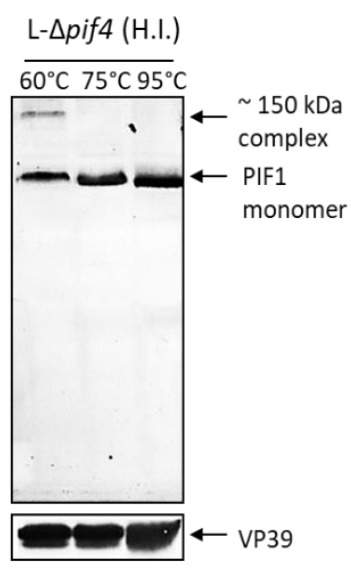

(b)

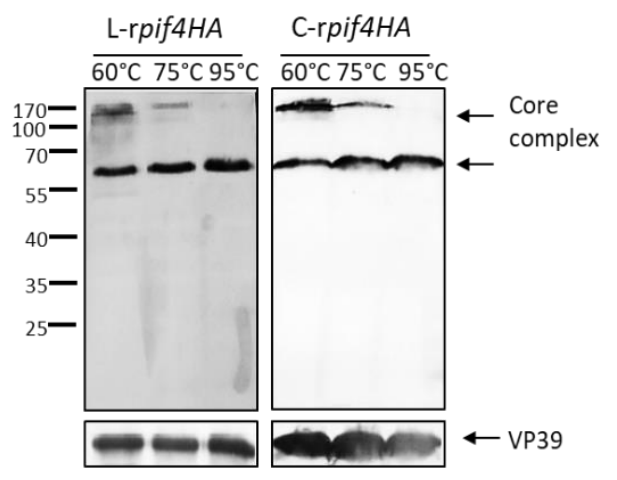

(c)

Figure 3: Western blot analysis of the PIF core-complex and PIF1 monomers in ODVs of the pif4 deletion and repair mutants. ODVs of the pif4 deletion mutant ( $\triangle$ pif4) were released from either C-OBs (a) or L-OBs with and without heat inactivation (b) for analysis with SDS-PAGE. The same analysis was performed with ODVs of the pif4 repair mutant (rpif4HA), released from C-OBs or L-OBs without heat treatment (c). For all samples, ODVs were purified, heated in Laemmli buffer at $60^{\circ} \mathrm{C}, 75^{\circ} \mathrm{C}$ or $95^{\circ} \mathrm{C}$ for 5 min and separated by SDS-PAGE. Western blot analysis was performed with PIF1 antiserum. Detection of capsid protein VP39 with VP39 antiserum was used as loading control (lower panels). The arrows indicate the full-sized $170 \mathrm{kDa}$ complex, $150 \mathrm{kDa}$ complex in the pif4 deletion- and repair mutant, and monomers of PIF1 and VP39. 
at $75^{\circ} \mathrm{C}$. However, when the deletion mutant was repaired with a HA-tagged pif4 gene, the 170 kDa complex and PIF1 monomers were detected again in the ODVs after heating at 60 and $75^{\circ} \mathrm{C}$, even without prior heat inactivation of the co-occluded proteases in L-OBs (Fig. 3c). Apparently, the smaller complex is degraded more easily by these proteases than the $\sim 170 \mathrm{kDa}$ complex that still contains PIF4. These observations indicate that PIF4 is an important stabilizing factor of the core-complex to resist proteolytic activity of host derived co-occluded proteases.

PIFO, PIF2 and PIF3 are also degraded by co-occluded proteases in L-ODVs of various pifdeletion mutants.

The results above show that PIF1 monomers are prone to proteolytic degradation by cooccluded proteases in L-ODVs after deletion of pifo- or pif4. To determine whether PIF1 monomers are also degraded in other pif-deletion mutants, ODVs of pif1-, pif2-, and pif3 deletion mutants were analysed for the presence of PIF1 monomers. As this research question only relates to PIF-protein monomers, all ODV-samples were fully denatured by heating at $95^{\circ} \mathrm{C}$ and analysed by western blot analysis with antiserum against PIF1. In C-ODVs, PIF1 monomers were found in all pif deletion mutants, except for the pifl deletion mutant, while PIF1 monomers in L-ODVs were only detected in wildtype and the pif4 repair mutant (Fig. 4a and b). However with ODVs released from heat-treated L-OBs, the PIF1 monomers were found again in all pif deletion mutants, except the pifl deletion mutant (Fig. 4c). Also some nonspecific bands of unknown origin were found of approximately $50 \mathrm{kDa}$, which are unlikely

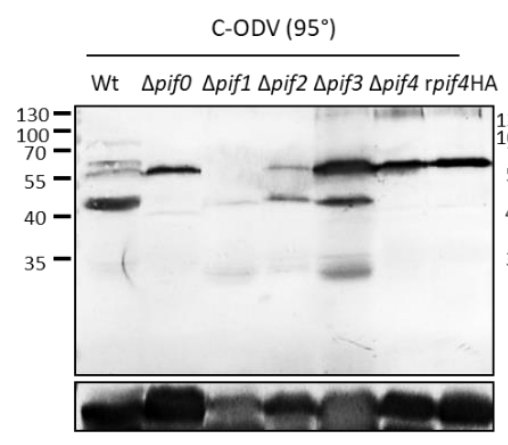

(a)

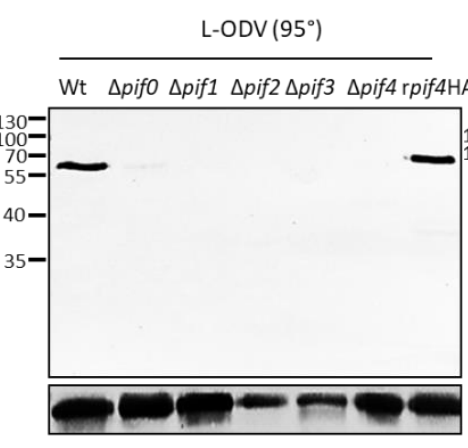

(b)

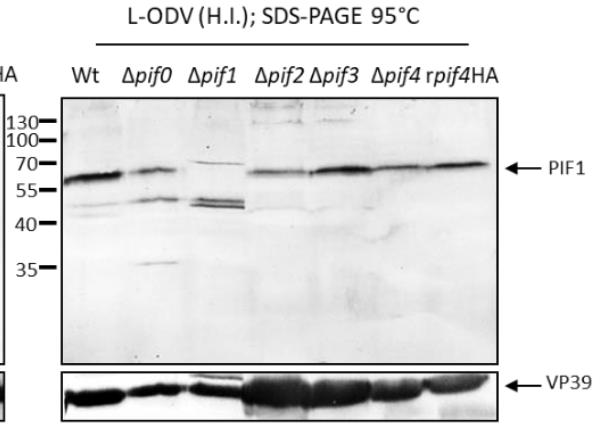

(c)

Figure 4: Western blot analysis of PIF1 monomers in ODVs of various pif deletion mutants, derived from cultured insect cells $(\mathbf{C})$ and infected larvae (L). ODVs were purified from a wildtype (Wt) construct, $\Delta$ pifo-, $\Delta$ pif1-, $\Delta$ pif2-, $\Delta$ pif3-, $\Delta$ pif4 mutants and the pif4 repair mutant (rpif4HA), heated in Laemmli buffer at $95^{\circ} \mathrm{C}$ for 5 min and separated by SDS-PAGE for western blot analysis with PIF1 antiserum. The ODVs were released from C-OBs (a), L-OBs (b) or heat-inactivated L-OBs (c). In the analysis with C-ODVs and heat inactivated L-ODVs, some nonspecific bands were also detected in some samples but are from unknown origin. Detection of capsid protein VP39 with VP39 antiserum was used as loading control (lower panel). 


\section{L-ODVs $\left(95^{\circ} \mathrm{C}\right)$}

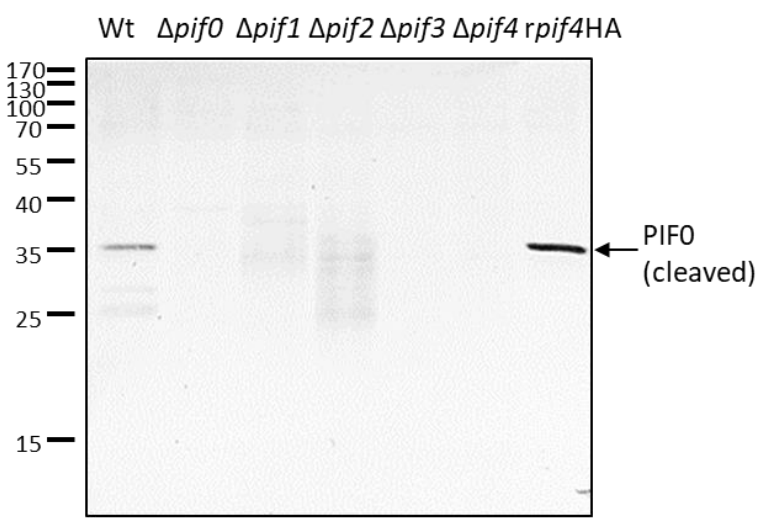

(a)

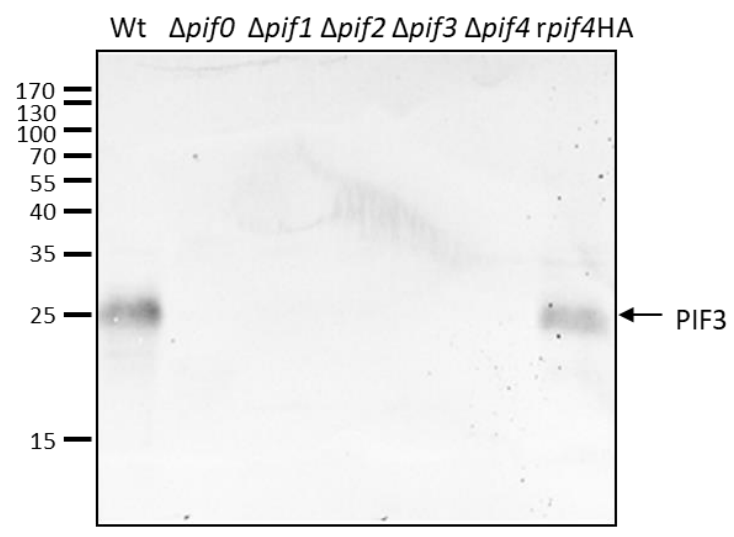

(c)

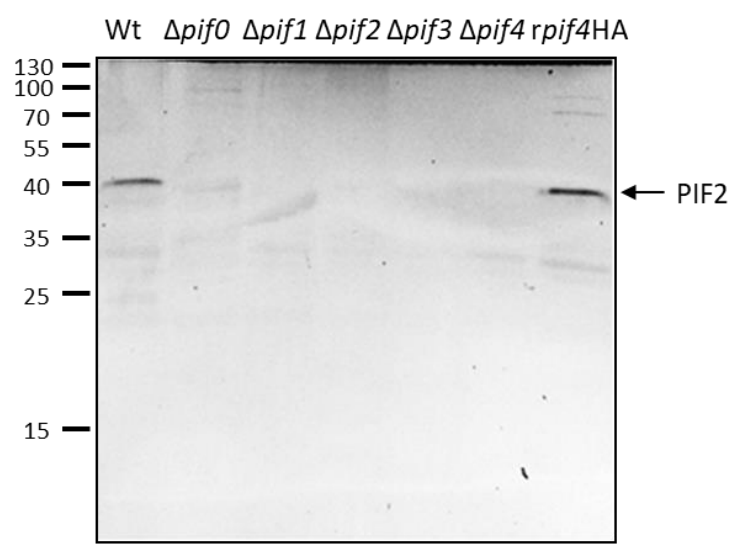

(b)

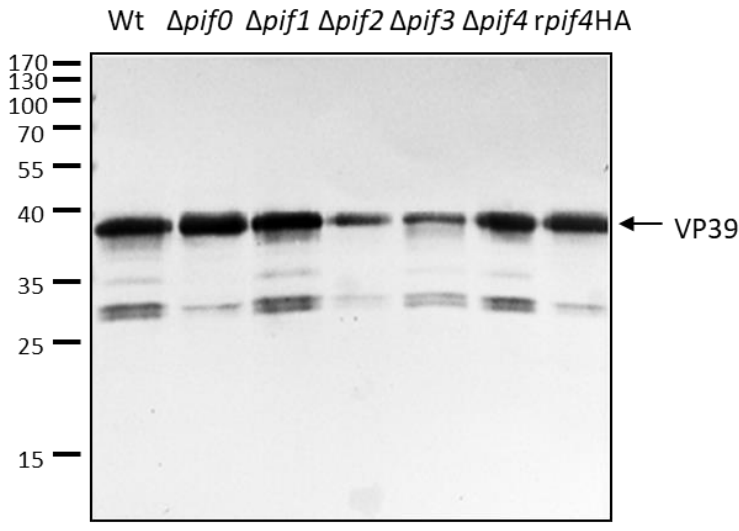

(d)

Figure 5: Western blot analysis of PIF-protein monomers on ODVs of various pif deletion mutants, released from L-OBs. ODVs were purified from L-OBs without prior heat treatment of a wildtype (Wt) construct, $\Delta$ pifo-, $\Delta$ pif1-, $\Delta$ pif2-, $\Delta$ pif3-, $\Delta$ pif4 mutants and the pif4 repair mutant (rpif4HA), heated in Laemmli buffer at $95^{\circ} \mathrm{C}$ for 5 min and separated by SDS-PAGE for western blot analysis with antiserum against PIF0 (a), PIF2 (b) and PIF3 (c). Detection of the major capsid protein VP39 with VP39 antiserum was used as loading control (d).

PIF1 degradation products as these bands were also detected in the pifl deletion mutant. The L-ODVs released from L-OBs without prior heat treatment were also analysed with antiserum against PIF0, PIF2 and PIF3 to assess whether these PIFs are degraded as well (Fig. 5). Just as previously observed with PIF1, these PIFs were only found in L-ODVs of wildtype and pif4 repair viruses and not in the pif deletion mutants, indicating that these PIFs are degraded as well. In ODVs of wildtype and the pif4 repair mutant, PIF2 and PIF3 were detected at the expected sizes of 40 and $25 \mathrm{kDa}$ respectively. PIF0 was detected at $35 \mathrm{kDa}$ rather than $74 \mathrm{kDa}$ due its cleavage by co-occluded host derived proteases as observed previously by Peng et al., 2011. These data indicate that not only PIF1, but also PIF0, PIF2 and PIF3 are proteolytically degraded by host derived proteases in the various pif-deletion mutants. 
PIF6 is a loosely associated component of the entry complex.

Prior research identified the protein AC68 as a PIF-protein (denoted PIF6) and as a possible interaction partner of PIF1 (Nie et al., 2012, Peng et al., 2012). Here the envelope proteins of a HA-tagged pif6-repair mutant were analysed under denaturing and non-denaturing conditions to determine whether PIF6 is part of the core-complex or is a relatively loosely associated component of the entry complex similar to PIF0. Under denaturing conditions, when samples were heated at $60^{\circ} \mathrm{C}$, anti-HA antibodies detected PIF6 only as $15 \mathrm{kDa}$ monomers and not in the complex (Fig. 6a, right panel). However, when the blot was treated with antiserum against PIF1, the $\sim 170 \mathrm{kDa}$ complex and PIF1 monomers were found as expected (Fig. 6a, left panel). PIF6 therefore does not appear to be part of the $170 \mathrm{kDa}$ core-complex. When the ODVenvelope proteins of the HA-tagged pif6 repair mutant were analysed under non-denaturing conditions, a double band was detected with antiserum against PIF1 and antibodies against the HA-tag just below the $480 \mathrm{kDa}$ marker, similar to what was observed with wildtype virus and

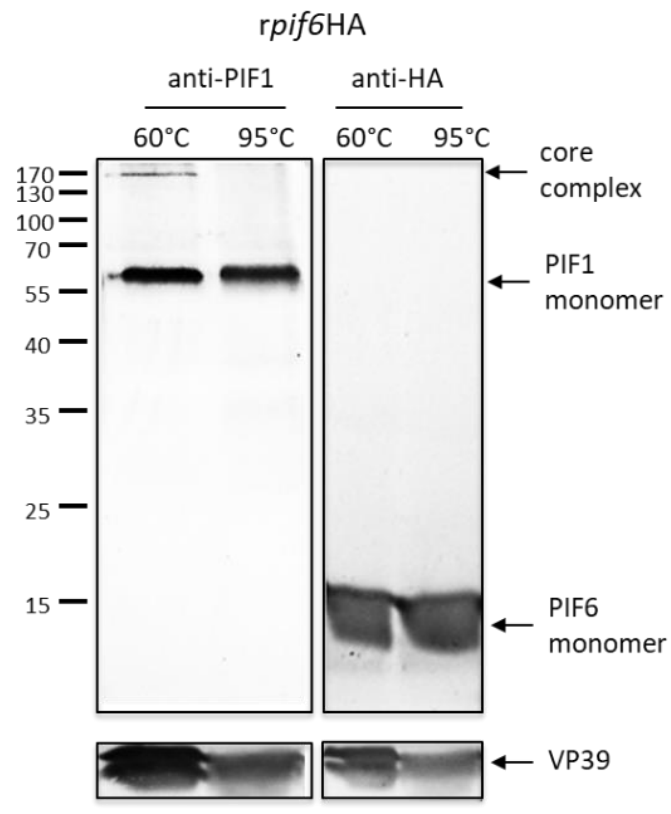

(a)

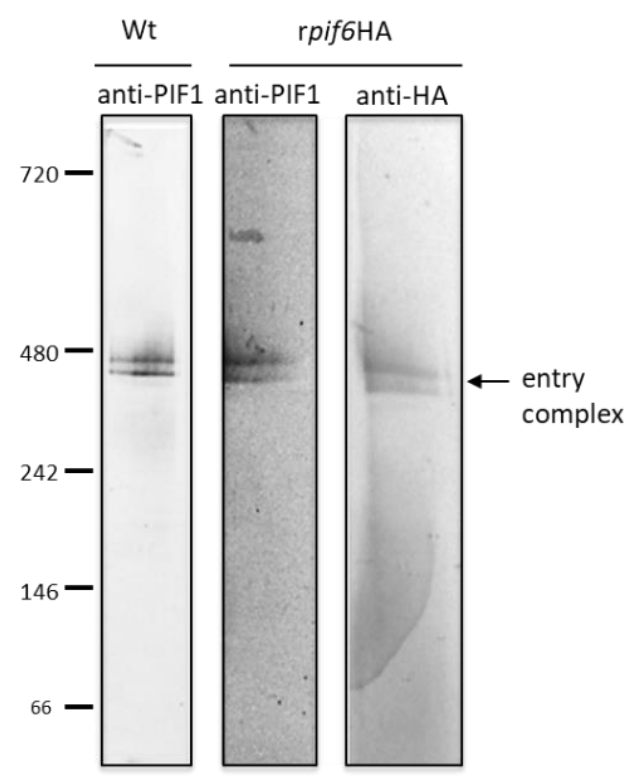

(b)

Figure 6: Western blot analysis of the PIF complex, PIF1- and PIF6 monomers under denaturing and nondenaturing conditions using a HA-tagged pif6 repair virus. ODV envelope proteins of the pif6 repair mutant (rpif6HA) were purified from ODVs by incubation in extraction buffer, containing $0.5 \%$ Triton, and analysed under denaturing (a) or non-denaturing conditions (b). For analysis under denaturing conditions, a sample of purified envelope proteins was heated in Laemmli buffer at $60^{\circ} \mathrm{C}$ or $95^{\circ} \mathrm{C}$ for $5 \mathrm{~min}$ and separated by SDS-PAGE for western blot analysis with antiserum against PIF1 (left panel of a) and the HA-tag of PIF6 (right panel of a). Detection of the capsid protein VP39 with anti-VP39 antiserum was used as loading control (lower panel of a). For analysis under non-denaturing conditions, a sample of ODV envelope proteins was separated on a 4-16\% Bis-Tris gradient gel for western blot analysis with antibodies against PIF1 and the HA-tag of PIF6 (b). The same analysis was done with the envelope proteins of wildtype (Wt) ODVs as positive control. 
anti-PIF1 antiserum in blue native-PAGE gels (Fig. 6b). These results show that PIF6 is also a loosely associated component of the entry complex.

PIF6 is important for the proteolytic resistance of the core-complex against co-occluded proteases.

As the PIF6 protein appears to be a loosely associated component of the entry complex, L-ODVs of a pif6 deletion mutant were analysed by SDS-PAGE and western blot analysis with and without prior heat treatment of L-OBs, to determine whether the presence of PIF6 is important for the proteolytic resistance of the core-complex and PIF monomers. When the cooccluded proteases in the L-OBs were inactivated by heat prior to dissolution, the core complex and PIF1 monomers were found after heating of the isolated ODVs at $60^{\circ} \mathrm{C}$ with antiserum against PIF1 (Fig. 7a). After heating at $95^{\circ} \mathrm{C}$, the complex was fully dissociated and only PIF1 monomers were found in the western blot analysis. When analysing L-ODVs without prior heat treatment, the anti-PIF1 antibodies only detected a pattern of degradation products below the $170 \mathrm{kDa}$ marker when heated at $60^{\circ} \mathrm{C}$, and the PIF1 monomers were absent (Fig. $\left.7 \mathbf{b}\right)$. When the complex was completely dissociated by heating at $95^{\circ} \mathrm{C}$, the PIF1 monomers were not detected either. However, when L-ODVs of the pif6-repair mutant were analysed, the corecomplex (together with degradation products) and PIF1 monomers were detected at $60^{\circ} \mathrm{C}$, and

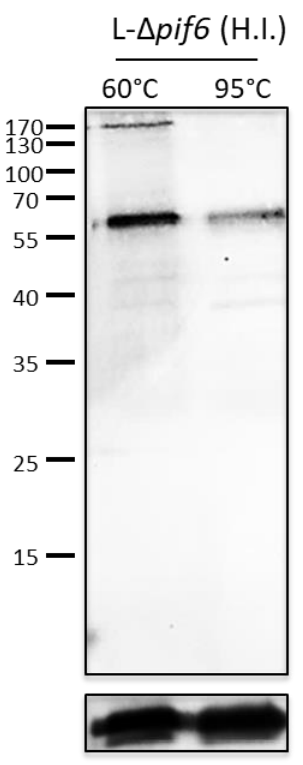

(a)

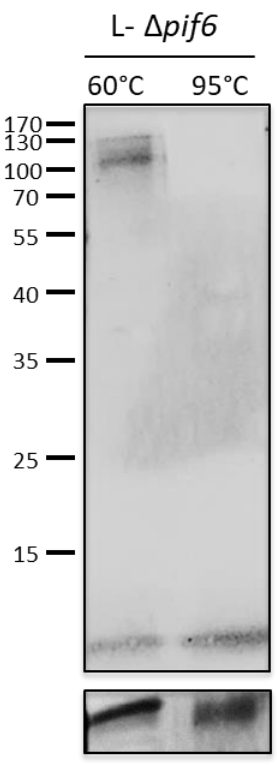

(b)

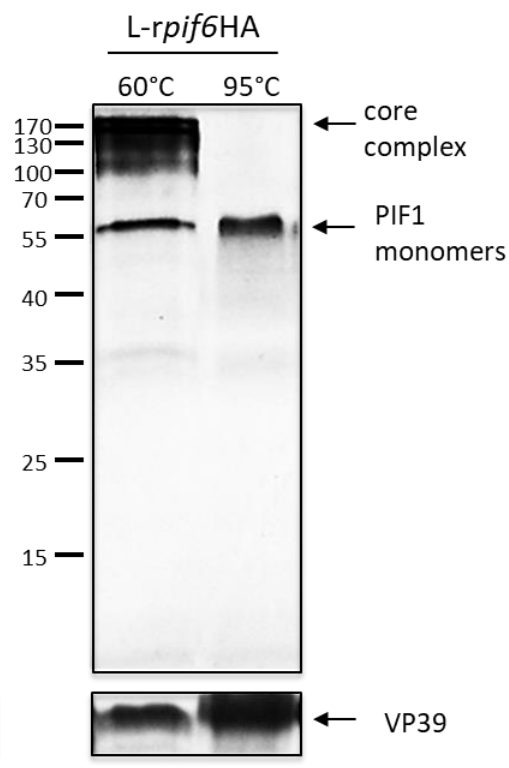

(c)

Figure 7: Western blot analysis of the PIF complex, PIF1- and PIF6 monomers in ODVs of the pif6 deletion and pif6 repair mutants. ODVs of the pif6 deletion mutant ( $\triangle$ pifo) were purified from L-OBs with (a) and without (b) heat inactivation (H.I.) and heated in Laemmli buffer at $60^{\circ} \mathrm{C}$ or $95^{\circ} \mathrm{C}$ for $5 \mathrm{~min}$ and separated by SDSPAGE. Western blot analysis was performed with PIF1 antiserum in. L-ODVs of the pif6 repair mutant without heat inactivation were also analysed with antiserum against PIF1 (c). Detection of capsid protein VP39 with antiVP39 antiserum was used as loading control (lower panels). 
only PIF1 monomers were detected upon heating at $95^{\circ} \mathrm{C}$ (Fig. 7c). These results indicate that PIF6 is important for the proteolytic resistance of the core-complex, despite being a loosely associated component of the entry complex.

\section{Discussion}

Various studies have shown that the proteins in ODVs released from L-OBs are affected by cooccluded host-derived proteases (Summers and Smith, 1975, Wood, 1980, Peng et al., 2011). Here we show that the baculovirus ODV-entry complex, consisting of a stable core-complex and at least four different loosely associated PIFs (PIF0, PIF7, PIF8 and now also PIF6), needs to be completely intact to resist proteolytic activity of these proteases during L-OB dissolution under alkaline conditions in vitro. Even in wildtype L-ODVs with an intact entry complex, we observed that the stable core-complex was largely degraded after treatment in Laemmli buffer at $50^{\circ} \mathrm{C}$, whereas the core remained largely intact after heating at $60^{\circ} \mathrm{C}$ or $75^{\circ} \mathrm{C}$. This observation can be explained by dissociation of the loosely associated components of the entrycomplex from the stable core due to the presence of SDS in the Laemmli buffer for SDS-PAGE analysis, leaving the core-complex more exposed to proteases. It also implies that the cooccluded proteases are still present in the protein sample after L-OB dissolution and that these are still active in SDS-containing Laemmli buffer at elevated temperatures. This is in accordance with previous reports about co-occluded proteases in various other baculoviruses, which showed that the proteolytic activity of the proteases was mainly associated with the virus particles (Payne and Kalmakoff, 1978), and that the proteases are thermostable and compatible with detergents as SDS and reducing agents (Summers and Smith, 1975, Payne and Kalmakoff, 1978, Tweeten et al., 1978). As such, when the isolated L-ODVs were incubated in Laemmli buffer at $50^{\circ} \mathrm{C}$, the proteases were likely still active and degraded the exposed core-complex and dissociated PIFs. In contrast, when the L-ODVs were heated at $60^{\circ} \mathrm{C}$ or $75^{\circ} \mathrm{C}$, the proteases appeared sufficiently inactivated as both the core-complex and PIF monomers were found again, as they were with C-ODVs. Consequently, to assess only the effects of the co-occluded proteases on PIFs during OB-dissolution in DAS-buffer (mimicking the alkaline environment of the larval midgut), we heated the protein samples at $60^{\circ} \mathrm{C}$ or higher to prevent further degradation during SDS-PAGE sample preparation. Although these experimental conditions are not biologically relevant, they can nevertheless be used to assess the importance of the different components of the entry complex with regard to the proteolytic activity of co-occluded proteases during $\mathrm{OB}$ dissolution in the larval midgut. The presented results provide some 
insight in the properties of the co-occluded proteases in L-OBs, but further characterisation of these proteases is still necessary to unravel the biological consequences and importance of their activities.

Analysis of a pifO deletion mutant initially suggested that PIFs in the core-complex are more resistant to co-occluded proteases than as monomer, as only the stable core-complex and not the PIF1 monomers were found in ODVs without prior heat treatment of the L-OBs. In contrast, the stable core-complex was found degraded in the pif4 and pif6 deletion mutants, indicating that the core-complex requires these components to maintain proteolytic resistance. It was previously shown that deletion of pif4 resulted in the formation of a smaller core-complex of PIF1, PIF2 and PIF3 (Peng et al., 2012). Apparently, PIF4 is not essential for forming the stable core, but it does seem important for the resistance of the core-complex against the proteolytic activity of co-occluded proteases. In contrast to PIF4, PIF6 proved not to be part of the corecomplex but was identified as a loosely associated component of the entry complex. Despite this more loose association with the core-complex, PIF6 appeared to be more important for the proteolytic stability of the core-complex than PIF0. Apparently, the importance of the various PIF-proteins for the proteolytic resistance of the core-complex can differ independently of whether the missing PIF-protein is a core component or a loosely associated component of the complex. To understand this aspect better, further information is needed about the proteinprotein interactions in the ODV entry complex and the stoichiometry of the individual components.

The importance of an intact entry complex in the ODV-envelope for the proteolytic resistance of PIF-proteins can also be nicely illustrated by the current observations on PIF0 monomers. PIF0 has previously been shown to be cleaved into two fragments of approximately $35 \mathrm{kDa}$ and $37 \mathrm{kDa}$ in L-ODVs (Peng et al., 2011). However, current study showed that deletion of any pifgene affected proper cleavage of PIF0 and resulted in proteolytic degradation of this PIF-protein instead. Apparently, the protein-protein interactions in the entry complex ensures that PIF0 is cleaved only at the directed cleavage site and prevent total degradation, but when a PIF-protein is missing due to deletion of a pif gene, co-occluded proteases are able to degrade PIF0 along with the other PIF-proteins.

The observations presented in this study indicate that deletion of a pif-gene affects the structure of the entry-complex which consequently leads to degradation of the remaining PIF-proteins by most likely co-occluded alkaline proteases during OB-dissolution. This finding conflicts 
with previous observations on binding and fusion properties of pif1-, pif2- and pif3 deletion mutants of AcMNPV with midgut epithelial cells in Heliothis virescens larvae (Ohkawa et al., 2005). These authors showed that deletion of pifl or pif 2 compromised binding and fusion of the L-ODVs with epithelial cells in the larval midgut, while the pif3 deletion mutant was still functional in terms of binding and fusion. In addition to PIF1 and PIF2, PIF0 has also been shown to be crucial for binding and fusion of ODVs to epithelial midgut cells (Haas-Stapleton et al., 2004). However, our study indicates that deletion of pif3 results in proteolytic degradation of PIF0, PIF1 and PIF2 under alkaline conditions, which possibly affects the binding and fusion capacities of ODVs in the larval midgut. Furthermore, PIF3 has been shown to be essential for formation of the stable core complex and was also shown to interact with PIF0 in AcMNPV (Peng et al., 2010). A recent yeast-two-hybrid study suggests that the interaction between PIF3 and PIF0 is indirect (Zheng et al., 2017), but this needs to be validated by further experiments. Nevertheless, deletion of pif 3 results in the inability to form the entry complex as a consequence of lost protein-protein interactions between the PIFs known to be involved in binding and fusion, and might additionally be degraded by co-occluded proteases. Consequently, it can be hard to draw firm conclusions about the involvement of a single PIF during the early stages of oral infection by making pif deletion mutants as the remaining PIFs in the ODV-envelope are severely affected by loss of protein-protein interactions and presence of host derived cooccluded proteases.

\section{Conclusions}

This study demonstrates the importance of an intact ODV entry complex to resist proteolytic activity of host-derived proteases that are co-occluded in L-OBs, as upon deletion of a pif gene the remaining PIFs are more sensitive to proteolytic degradation. This observation could be biologically relevant as in the alkaline environment of the midgut, the co-occluded proteases can be activated as did the alkaline buffer in our in vitro experiments. But even when the cooccluded proteases are not activated in the larval midgut, proteolytic resistance is still important as the alkaline proteases normally present in the midgut lumen can supplement the co-occluded proteases. Thus, proteolytic resistance against proteases can be considered as an important selective pressure, explaining the high level of conservation of PIF-proteins among the different baculoviruses in order to maintain complex formation and preserve oral infectivity. 


\section{Material and methods}

Culture of insect cells and lepidopteran larvae. Sf21 cells were cultured in T25 flasks with Graces medium (Fisher N.V.), supplemented with $10 \%$ fetal bovine serum (FBS) and $50 \mu \mathrm{g} / \mathrm{ml}$ gentamycin. Sf21 suspension cultures were grown in Sf900II medium (Fisher N.V.) supplemented with 5\% FBS and $50 \mu \mathrm{g} / \mathrm{ml}$ gentamycin. Spodoptera exigua larvae were reared on artificial diet in a $26^{\circ} \mathrm{C}$ climate room with $40 \%$ humidity and a $16: 8 \mathrm{~h}$ (light:dark) photoperiod.

Bacmids. The AcMNPV bacmid bMON14272 was derived from the Bac-to-Bac system (Invitrogen; Luckow et al., 1993). The construction of the bacmids with the individual pif-gene deletions (del-pif0, del-pif1, del-pif2 and del-pif4) has been described previously (Peng et al., 2010, 2011, 2012). The pif3 deletion bacmid was kindly provided by Dr Xinwen Chen of the Wuhan Insitute of Virology and has been described before in Li et al., 2007. The construction of the HA-tagged pif4 repair bacmid has also been described previously (Fang et al., 2009, Peng et al., 2012). The bacmids of the ac68 (pif6) deletion mutant and the corresponding HA-tagged repair construct has also been described previously (Nie et al., 2012). In all deletion and repair bacmids, the polyhedrin gene was restored to enable the resulting virus to form OBs. The polyhedrin (polh) gene was restored by using the Bac-to-Bac transposition protocol with a pFastBacDual vector (Invitrogen) from which the p10 promotor was deleted and the ORF of the polh gene was inserted behind the polyhedrin promotor as previously described (Peng et al., 2010). The pif ORFs needed to repair the deletion bacmids were introduced simultaneously with the polh gene and were placed under control of their native promoters.

Virus proliferation and purification. Sf21-cells were transfected with the bacmids using Expres2TR reagent (Expres2ion Biotechnologies) to generate first generation BV-stocks. Subsequently, the obtained BV-stocks were amplified in Sf21 cells by an additional round of infection at low MOI. Cell-derived OBs (C-OBs) were generated by infecting a $80 \mathrm{ml}$ suspension cultures of Sf21-cells at $1.5 \times 10^{6}$ cells $/ \mathrm{ml}$ with $1 \mathrm{ml}$ of amplified BV-stock (approximate MOI of 1) and incubated for a week. After a week of incubation, the cells were pelleted by centrifugation at $4000 \mathrm{x}$ g for $30 \mathrm{~min}$. The cells were suspended in $0.1 \%$ SDS dissolved in demi water and incubated for $2 \mathrm{~h}$ at $37^{\circ} \mathrm{C}$ with gentle agitation. Then the cells were sonicated for 1 min at 7 Watt. Total cell lysis was verified by light microscopy and the OBs were pelleted by centrifugation at $4000 \mathrm{x} \mathrm{g}$ for $30 \mathrm{~min}$. Larva-derived OBs (L-OBs) were obtained by injection of $1 \mu \mathrm{l}$ of the amplified BV-stock into the haemocoel of L4 Spodoptera 
exigua larvae, using an Humapen Luxura insulin pen (Lilly) $\left(\mathrm{TCID}_{50} \sim 5 \times 10^{7}-1 \times 10^{8}\right.$ per ml). Three to four days post injection, the liquefied larvae were collected, homogenized in sterilized water and filtered through two layers of cheese cloths. The debris in the flow through was pelleted by centrifugation at $500 \mathrm{x} \mathrm{g}$ for $10 \mathrm{~min}$ and the L-OBs in the supernatant were subsequently harvested by centrifugation at $4000 \mathrm{x} \mathrm{g}$ for $30 \mathrm{~min}$. The pellet of the first centrifugation step, containing the cell debris, was washed multiple times with water followed by centrifugation to further purify the OBs. Finally, the purified OBs were washed twice in sterilized water. Both C-OBs and L-OBs were further purified by centrifugation over a $30 \%$ $60 \%(\mathrm{w} / \mathrm{w})$ sucrose gradient in a Beckmann SW32 rotor at $90,000 \mathrm{x}$ for $1 \mathrm{~h}$ at $4^{\circ} \mathrm{C}$. The band with OBs was withdrawn from the gradient with a Pasteur pipette and subsequently pelleted by centrifugation at $4000 \mathrm{x} \mathrm{g}$ for $30 \mathrm{~min}$.

SDS-PAGE and Western blot analysis. ODVs were released from approximately $3.0 \times 10^{8} \mathrm{OBs}$ by treatment with DAS buffer $\left(0.1 \mathrm{M} \mathrm{Na}_{2} \mathrm{CO}_{3}, 166 \mathrm{mM} \mathrm{NaCl}\right.$ and $10 \mathrm{mM}$ EDTA, $\left.\mathrm{pH} 10.5\right)$ for 10 minutes at $37^{\circ} \mathrm{C}$. When endogenous alkaline proteases needed to be inactivated, L-OBs were heated at $80^{\circ} \mathrm{C}$ for $40 \mathrm{~min}$ prior to dissolution in alkaline DAS-buffer. After verification of OB dissolution by light microscopy, the ODV suspension was neutralized with 1/10 volume of $0.5 \mathrm{M}$ Tris- $\mathrm{HCl} \mathrm{pH}$ 7.5. Non-dissolved debris was removed by centrifugation at $1500 \mathrm{x} \mathrm{g}$ for 2 min and the ODVs in the supernatant were pelleted by centrifugation at 20,800 x g for $25 \mathrm{~min}$ at $4^{\circ} \mathrm{C}$. The ODVs were resuspended overnight with gentle rotation at $4^{\circ} \mathrm{C}$ in $0.1 \mathrm{x}$ TE buffer, supplemented with Complete Protease Inhibitor Cocktail (Roche). Then 4x Laemmli buffer was added to the ODV-suspension which was then heated at $50^{\circ} \mathrm{C}, 60^{\circ} \mathrm{C}, 75^{\circ} \mathrm{C}$ or $95^{\circ} \mathrm{C}$ for 5 minutes. The samples were separated in a 12\% SDS-PAGE gel and blotted onto a PVDF membrane (Milipore Immobilon-P). Western blot analyses with antisera against various PIFproteins were performed as previously described in Peng et al., 2010. In brief, anti-PIF0 antiserum (1:1000 dilution), produced in rabbit, and anti-PIF1 (1:2000 dilution), anti-PIF2 (1:1000 dilution) and anti-PIF3 antisera (1:1000 dilution), all produced in rats, were used as primary antibodies. To detect the HA-tagged PIF6 in the pif6-repair mutant, anti-HA antibodies were used as primary antibody (1:2000; Roche 3F10). Detection of capsid protein VP39 with anti-VP39 antibodies (1:1000), produced in mice, was used as loading control. Goat anti-rabbit (1:2000 dilution; Dako), goat anti-rat (1:2000 dilution; Sigma A8438) and goat anti-mouse antibodies (1:2000 dilution; Sigma A5153), conjugated to alkaline phosphatase, were used as secondary antibodies to detect the proteins of interest by conversion of NBT-BCIP substrate 
(Sigma) in a blue-purple coloured precipitant in AP-buffer (0.1M Tris- $\mathrm{HCl}, 0.1 \mathrm{M} \mathrm{NaCl}, 5 \mathrm{mM}$ $\mathrm{MgCl}, \mathrm{pH} 10.5)$

Blue-native PAGE. ODVs of $3.0 \times 10^{8}$ OBs were isolated as described above. The ODV pellet was resuspended in $100 \mu \mathrm{l}$ extraction buffer (6.25 mM Tris, $37.5 \mathrm{mM} \mathrm{NaCl}, 0.5 \%$ Triton-X100, $\mathrm{pH}$ 7.2) and incubated overnight at $4^{\circ} \mathrm{C}$ with gentle rotation to extract the ODV envelope proteins. The envelope fraction was separated from the nucleocapsids by centrifugation at $16,000 \mathrm{x}$ g for $30 \mathrm{~min}$ at $4^{\circ} \mathrm{C}$. The supernatant containing the envelope fraction, was collected and mixed with 4x BN-PAGE sample buffer (200mM Bis-Tris, 64mM HCl, 200mM NaCl, $40 \%$ glycerol, $0,004 \%$ Ponceau S, pH 7.2). The protein samples were supplemented with 5\% Coomassie G-250 to a final concentration of $0.1 \%$. Electrophoresis of 4-16\% Bis-Tris gradient gels was performed as described in the manual for the NativePAGE ${ }^{\mathrm{TM}}$ Novex $^{\circledR}$ Bis-Tris Gel system (Invitrogen). NativeMark ${ }^{\mathrm{TM}}$ unstained protein ladder (Invitrogen LC0725) was also stained with Coomassie and used as marker. Blotting to a PVDF membrane was performed with NuPAGE® Transfer buffer (Invitrogen) according to the manufacturer's protocol.

\section{Acknowledgements}

This work was supported by grant 824.14.16 of the Netherlands Organization for Scientific Research (NWO). Els Roode and Hanke Bloksma are acknowledged for rearing the insects, culturing cells and their technical assistance in the lab. 


\title{
Chapter 4
}

\section{The baculovirus AC108 protein is a per os infectivity factor and a component of the ODV entry complex}

Bob Boogaard, Felix Evers, Jan W. M. van Lent, Monique M. van Oers

\begin{abstract}
Baculoviruses orally infect insect larvae when they consume viral occlusion bodies (OBs). OBs consist of a crystalline protein matrix in which the infectious virus particles, the occlusionderived viruses (ODVs), are embedded. The protein matrix dissolves in the alkaline environment of the insect's midgut lumen. The liberated ODVs then infect midgut epithelial cells through the action of at least nine different ODV-envelope proteins, called per os infectivity factors (PIFs). These PIF proteins mediate ODV oral infectivity, but are not involved in the systemic spread of the infection by budded viruses (BVs). Eight of the known PIFs form a multimeric complex, named the ODV entry complex. In this study, we show for the Autographa californica multiple nucleopolyhedrovirus that mutation of the ac108 open reading frame abolishes the ODV oral infectivity, while production and infectivity of the BVs remains unaffected. Furthermore, repair of the ac108 mutant completely recovered oral infectivity. With an HA-tagged repair mutant, we were able to demonstrate by western blot analysis that the AC108 protein is a constituent of the ODV entry complex, which formation was abolished in absence of this protein. Based on these results, we conclude that ac108 encodes a per os infectivity factor (PIF9) that is also an essential constituent of the ODV entry complex.
\end{abstract}

This chapter has been published as:

Boogaard, B., Evers, F., van Lent, J. W. M., \& van Oers, M. M. (2019). The baculovirus AC108 protein is a per os infectivity factor and a component of the ODV entry complex. Journal of General Virology, 100, 669-678. 


\section{Introduction}

The family Baculoviridae harbours a group of arthropod-specific viruses that infect the larval stages of lepidopteran, hymenopteran and dipteran insect species. Baculoviruses have circular double-stranded DNA genomes with 90 -180 open reading frames (ORF). Most baculoviruses have a biphasic replication cycle in which two different virus phenotypes are produced, budded viruses (BVs) and occlusion-derived viruses (ODVs) (see review by Williams et al., 2017). BVs are formed during the replication cycle when a (newly formed) nucleocapsid buds through the plasma membrane of an infected host cell. This phenotype is responsible for systemic spread of the viral infection in the larvae. Later in the replication cycle, ODVs are formed in the nucleus of the infected host cell, when one or more nucleocapsids are enveloped by a membrane that is derived from the inner nuclear membrane (Shi et al., 2015). The virions of the ODV phenotype are embedded in a crystalline protein matrix of either polyhedrin or granulin to form occlusion bodies (OBs). This protein matrix protects the ODVs against detrimental influences of the environment after their dispersal.

Insect larvae are orally infected by ODVs when they eat from OB contaminated food-sources. After ingestion of the OBs, the protein matrix dissolves in the highly alkaline milieu of the insect's midgut lumen, liberating the ODVs. The released ODVs infect midgut columnar epithelial cells, which requires a specific set of viral proteins called per os infectivity factors (PIFs). These proteins are located in the ODV-envelope and nine different PIFs have been identified to date (reviewed in Chapter 2). Three of these PIFs, PIF0, 1 and 2, are involved in binding of the ODVs to the brush border of the epithelial cells, but the biological role of the other PIFs is still enigmatic (Haas-Stapleton et al., 2004, Ohkawa et al., 2005). In the prototype baculovirus Autographa californica multiple nucleopolyhedrovirus (AcMNPV), eight of these PIFs have been found to form a large complex, named the ODV entry complex. This complex consists of a stable core, formed by PIF1, 2, 3 and 4 and four more loosely associated PIFs: PIF0, 6, 7 and 8 (Peng et al., 2010, 2012, Javed et al., 2017, Chapter 2, Chapter 3). The core complex is regarded as rather stable as it was still detected by western blot analysis with antiserum against one of its components after partial denaturation of the ODVs (Peng et al., 2010). The other components of the entry complex (PIF0, 6, 7 and 8) were only found as part of the entry complex under non-denaturing conditions and are therefore regarded as loosely associated components as these PIFs apparently associate with the core with lower affinity than the core components themselves. All these components of the entry complex have also been identified as interaction partners of PIF1 in a co-immunoprecipitation study (Peng et al., 2012). 
In such a co-immunoprecipitation study, AC108 was also identified as a possible interaction partner of PIF1, suggesting that this protein might be involved in oral infectivity as well. The Spodoptera frugiperda MNPV (SfMNPV) homolog Sf58 has been shown to be essential for ODV oral infectivity, while its homolog in Bombyx mori NPV (Bm91) appeared as nonessential (Simon et al., 2012, Tang et al., 2013). To determine whether AC108 is involved in oral infectivity, we constructed and analysed an AcMNPV ac108 mutant, as well as repaired viruses. The analyses of these viruses revealed that AC108 is crucial for ODV oral infectivity and that this protein is a component of the ODV entry complex.

\section{Results}

\section{Construction of ac108 mutant and repair bacmids}

To inactivate the ac108-gene in the AcMNPV-bacmid bMON14272 without affecting surrounding genes, we deleted a 12 nt sequence from -8 to +4 relative to the $A(+1)$ of the translational start codon by inserting a chloramphenicol acetyl transferase (cat) resistance gene, flanked by modified loxP-sites, via homologous recombination in E. coli, as described before (Datsenko and Wanner, 2000). The cat-gene was later removed by a CRE-lox reaction, leaving a $100 \mathrm{nt}$ insertion that is composed of fused loxP-sites (Suzuki et al., 2005). The insertion and subsequent removal of the cat-gene was confirmed by PCR analysis with primers that annealed

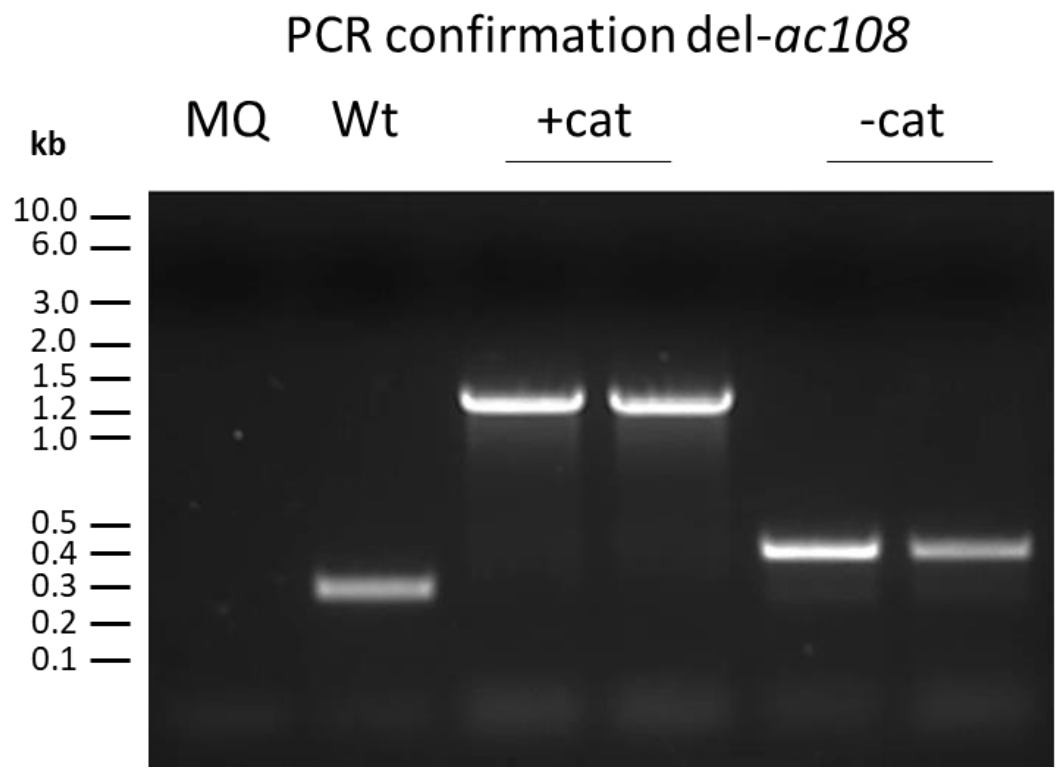

Figure 1: Conformation of insertion and removal of the chloramphenicol acetyl transferase (cat) resistance gene in the ac108-locus of the AcMNPV bMON14272-bacmid by PCR analysis. Primers were used that annealed outside the recombined region. The addition of double distilled water (MQ) as template served as a negative control for the PCR reaction and did not result in the formation of a PCR-product. Presence of the catgene is indicated as + cat and its absence as - cat. 


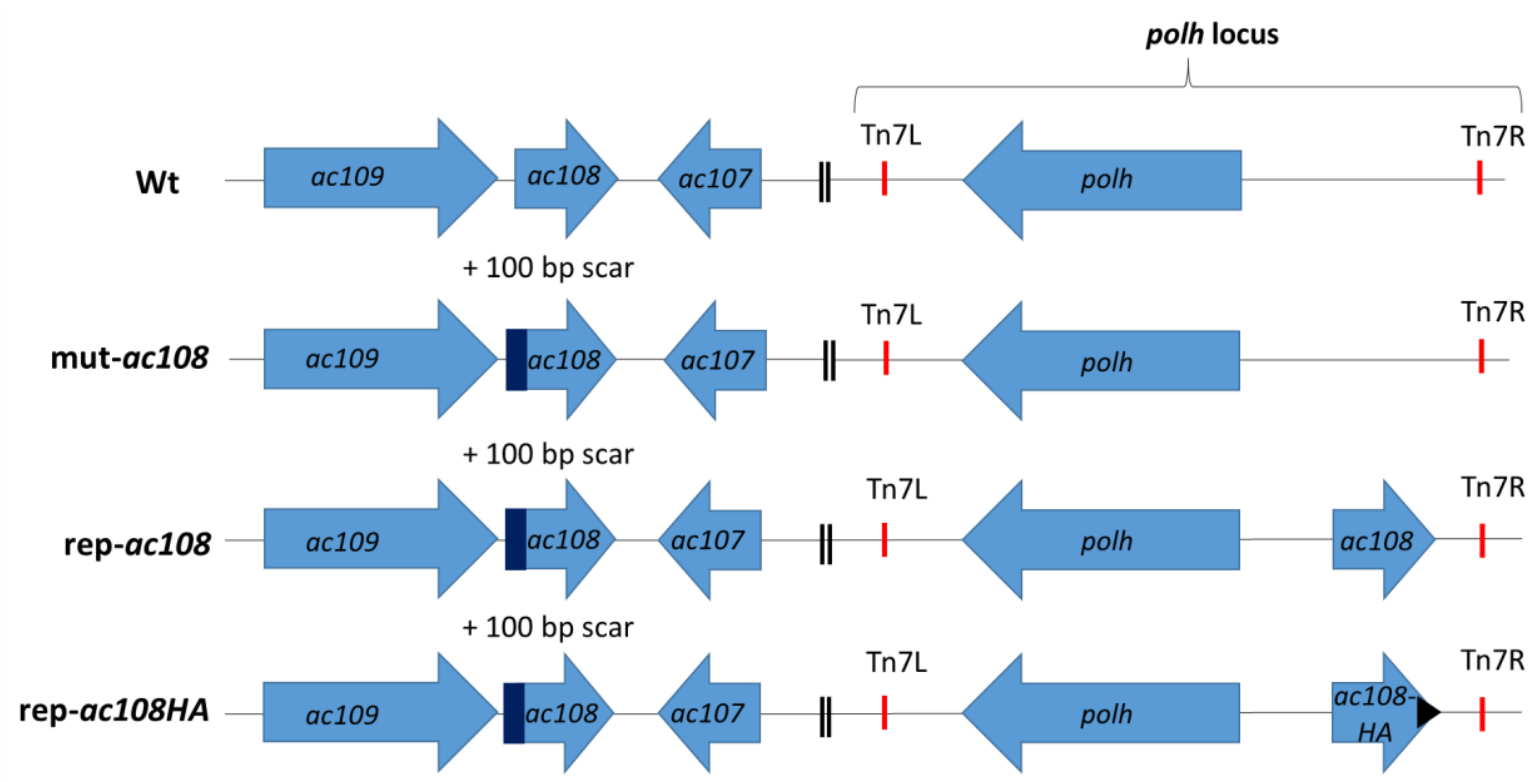

Figure 2: Schematic overview of the constructed AcMNPV bacmids. The ac108-gene was inactivated (mutac108) by deletion of a 12-nucleotide sequence from -8 to +4 relative to the $\mathrm{A}(+1)$ of the translational start codon. After removal of the cat-gene, a 100 bp scar was left, which is indicated in dark blue. The polyhedrin gene (polh) was restored by Tn7-mediated transposition with a modified pFBD-polh vector. The ac108 mutant bacmid was repaired by transposition with a pFBD-polh vector that also contained the ac108 putative promotor and ORF (pFBD-polh-ac108) or with such a vector that contained an HA-tagged ac108 ORF (pFBD-polh-ac108HA). This resulted in the ac108 repair (rep-ac108) and HA-tagged ac108 repair (rep-ac108HA) bacmids.

outside the recombined region (Fig. 1). When the wild type AcMNPV bacmid (Wt) was used as template, a PCR-product of approximately $300 \mathrm{bp}$ was produced. Insertion of the cat-gene (of approximately $1200 \mathrm{bp}$ ) in the ac108-locus (+cat) resulted in the formation of a $1500 \mathrm{bp}$ PCR product and after removal of this gene (-cat), leaving a 100 bp scar, a 400 bp PCR product was produced. To enable the production of OBs by the resulting ac108 mutant virus, the function of the polyhedrin (polh) gene was restored by Tn7-mediated transposition with a modified pFastBac Dual vector from that lacked the p10-promoter and in which the polh-ORF was inserted behind its own promoter (Peng et al., 2010) (Fig. 2). The original, non-mutated bacmid was also provided with a polh-ORF and is used as the wild type control in this study. The ac108 mutant bacmid was repaired via Tn7-mediated transposition with the modified pFastBac Dual vector that now also contained the ac108 promoter and ORF (with or without C-terminal HA-tag). This ORF was inserted between the NcoI and SphI restriction sites, in opposite direction of the polh-ORF (Fig. 2).

The ac108 mutant virus produces OBs and BVs as wild type.

The constructed ac108 mutant and repair bacmids were used for the transfection of Sf 21 cells to produce the modified viruses. For all these viruses, the transfected cells were found to 
(a)

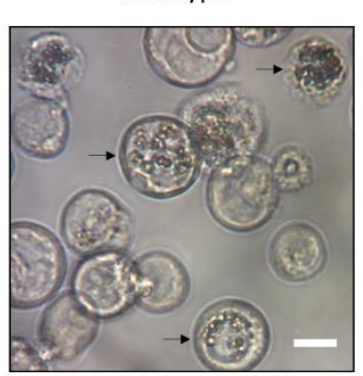

(b)

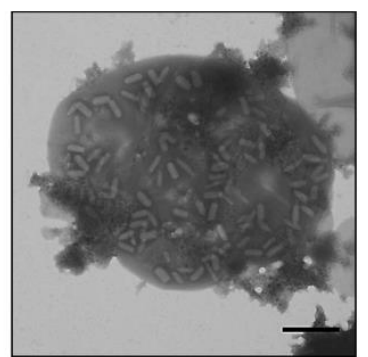

mut-ac108
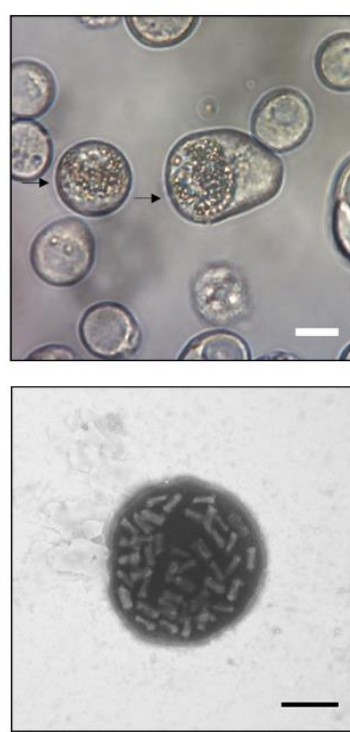

rep-ac108
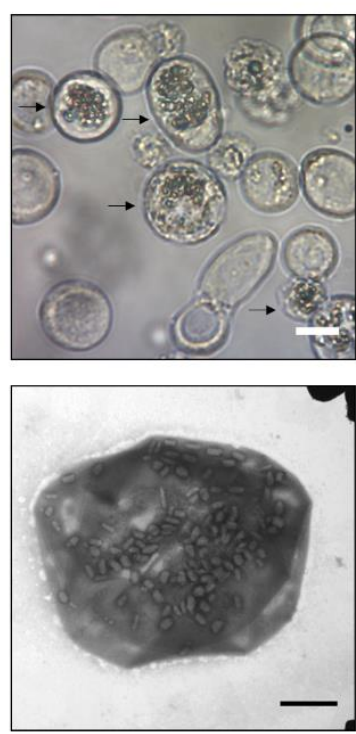

rep-ac108HA
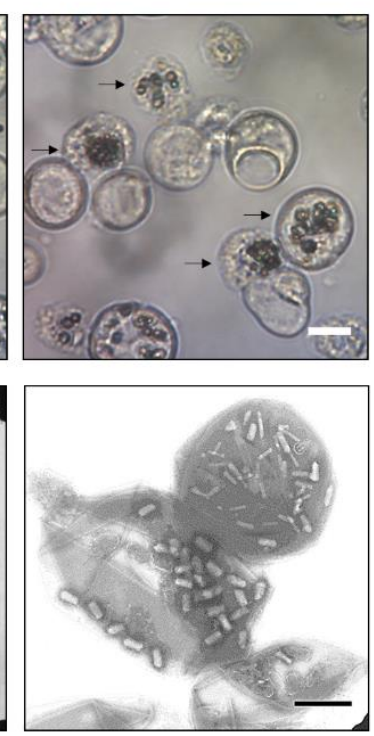

Figure 3: Microscopic analysis of OBs produced by the mutant viruses. (a) Sf21 cells at six days post transfection with the constructed bacmids of either wild type, ac108 mutant (mut-ac108), ac108 repair (repac108) or HA-tagged ac108 repair (rep-ac108HA) viruses. The black arrows indicate the OB containing cells and the white scale bar corresponds with $10 \mu \mathrm{m}$. (b) Electron microscopic analysis of the OBs to validate the presence of ODVs.

produce OBs as can be seen in light microscopic images taken six days post transfection (Fig. 3a). When the OBs were dissolved in alkaline buffer on copper grids for electron microscopic analysis, it was found that the OBs of the ac108 mutant contained ODVs, just as the repair mutants and the wild type (Fig. 3b). Furthermore, the mutant and repair viruses produced similar amounts of OBs as the wild type (Supplementary data). These findings indicate that the AC108 protein is not crucial for OB-production, nor for embedding of ODVs in the protein matrix of the OBs.

$\mathrm{BV}$ production of the mutant and repair viruses was assessed by constructing one-step BV growth curves. For that Sf9-ET cells, which produce green fluorescent protein (GFP) upon baculovirus infection (Hopkins and Esposito, 2009), were infected with BVs of the wild type, ac108 mutant or repair viruses at an MOI of 10, and the BV-titres of the medium were determined at 0, 24, 48 and 72 hours post infection (hpi) by end point dilutions assays. The BVtitres were found to increase every 24 hrs with approximately 1-log unit for the ac108 mutant and repaired viruses, just as observed with wild type (Fig. 4). At 48 and 72 hpi, infection with the ac108 mutant seemed to result in higher BV titres than infection with the other viruses, but this difference was not statistically significant (one way ANOVA; 48 hpi: $F(3,4)=2.88 \mathrm{p}=0.17$; 72 hpi: $F(3,4)=1.22 \mathrm{p}=0.41)$. From these results, it was concluded that the mutation in the ac108-locus did not affect the production of OBs and BVs. 


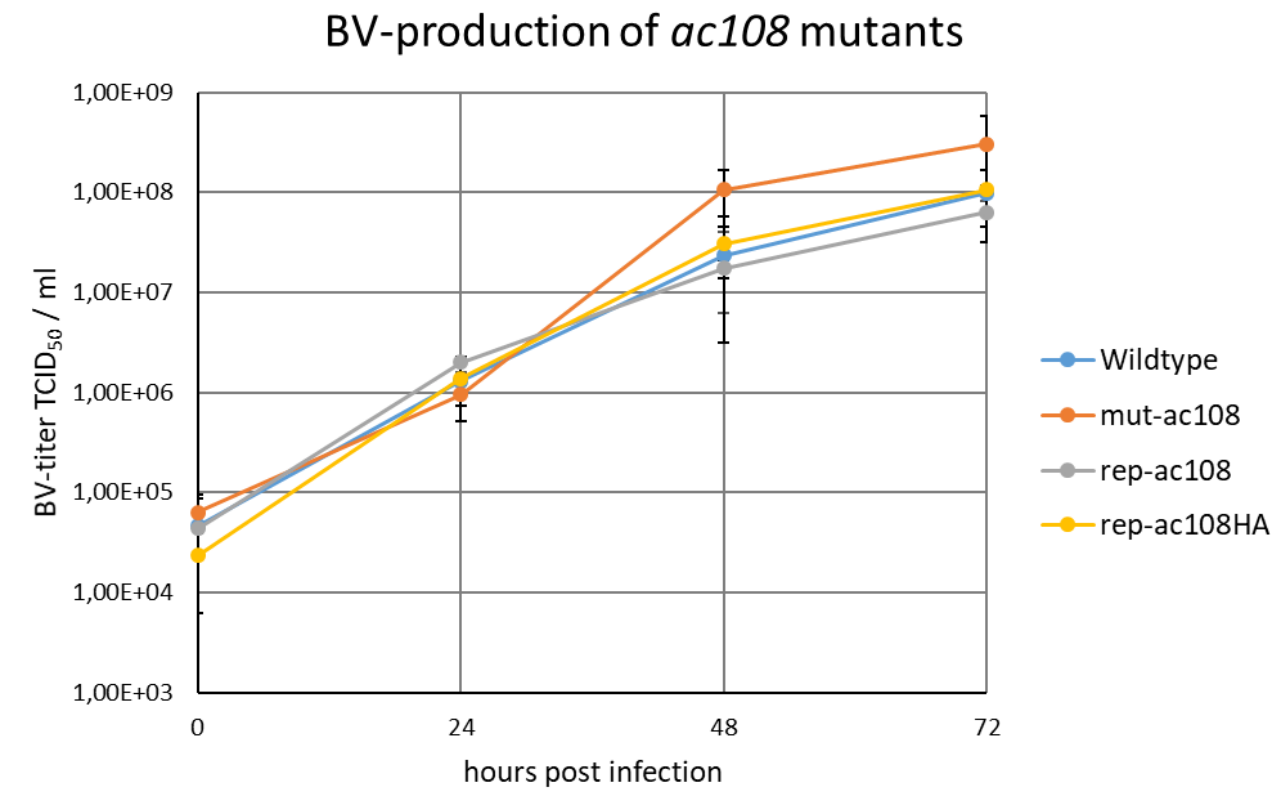

Figure 4: BV-growth curves after infection of Sf21 cells with BVs wild type AcMNPV, the ac108 mutant (mut-ac108), ac108 repair mutant (rep-ac108) or HA-tagged ac108 repair mutant (rep-ac108HA). The BV concentration increased approximately 1-log unit every 24 hrs for all mutants, just as observed with wild type, indicating that $\mathrm{AC} 108$ is not involved in $\mathrm{BV}$-production.

Inactivation of ac108 affects the oral infectivity of ODVs.

OBs of the mutant and repair viruses were fed by droplet feeding to Spodoptera exigua larvae to test the impact of the mutation in the ac108-ORF on the oral infectivity of ODVs. The droplet feeding assays were performed with a concentration of $3 \times 10^{8} \mathrm{OBs} / \mathrm{ml}$ on early L3 larvae that had been starved overnight. When OBs of the ac108 mutant were fed, the larvae did not show any mortality (Table 1). However, when the larvae were fed with OBs of the ac108 repair mutants (with or without HA-tag), they were successfully infected with similar mortality rates as the wild type. These data indicate that $a c 108$ is essential for the oral infectivity of ODVs in S. exigua larvae.

In parallel, we tested whether the mutation in the ac108-locus affected cell-to-cell transmission in the larvae by injecting BVs into the hemocoel. When the midgut was bypassed in this way, all larvae were infected with $100 \%$ mortality. This indicates that, in contrast to the ODVs, the

Table 1. Mortality after infection with $a c 108$ mutant and repair viruses

\begin{tabular}{lllllll}
\hline \multicolumn{2}{l}{ Droplet feeding assays } & $\left(3.0 \times 10^{8}\right.$ & $\mathrm{OBs} / \mathrm{ml})$ & \multicolumn{4}{l}{ Injection of BVs $\left(3.5 \times 10^{7} \mathrm{TCID}_{50} / \mathrm{ml}\right)$} \\
\hline mock & $0 / 12$ & $0 / 12$ & $0 / 11$ & mock & $0 / 24$ & $0 / 12$ \\
mut-ac108 & $0 / 12$ & $0 / 15$ & $0 / 12$ & mut-ac108 & $24 / 24$ & $12 / 12$ \\
rep-ac108 & $11 / 12$ & $17 / 17$ & $11 / 12$ & rep-ac108 & $24 / 24$ & $12 / 12$ \\
rep-ac108HA & $10 / 12$ & $19 / 19$ & $12 / 12$ & rep-ac108HA & $24 / 24$ & $12 / 12$ \\
wild type & $8 / 12$ & $12 / 15$ & $11 / 12$ & wild type & n.d. & n.d. \\
\hline
\end{tabular}


$\mathrm{BV}$ s retain their infectivity after mutation of the ac 108-gene. Hence, the AC108 protein appears to be essential for primary infection of the midgut epithelial cells by ODVs, but is not required for subsequent systemic infection by BVs.

AC108 is present in BVs and ODVs.

To determine whether AC108 is a structural component of ODVs, and potentially also of BVs, both virion phenotypes of the HA-tagged repair mutant were fractionated into envelope (E) and nucleocapsid $(\mathrm{N})$ fractions and analysed by western blotting with anti-HA antibodies. With these antibodies, AC108 monomers were detected as $15 \mathrm{kDa}$ polypeptides in the envelope and nucleocapsid fractions of BVs and ODVs. In both types of virus particles, this protein appeared to be enriched in the envelope fraction (Fig. 5a). When the fractionation of BVs and ODVs was validated by analyses with antisera against either the major capsid protein VP39, the ODV envelope protein PIF1 or the BV envelope protein GP64, it appeared that the fractionation of the virus particles was not completely efficient (Figs. 5b-c). When the fractionated BVs and ODVs were analysed with VP39 antiserum, the $39 \mathrm{kDa}$ monomers were also found in small quantities in the envelope fraction of the ODVs. VP39 was not detected in the BV envelope fraction, as expected (Fig. 5b). Furthermore, when the BV fractions were analysed with GP64 antiserum, this BV envelope protein was not only detected in the envelope fraction, but also in the nucleocapsid fraction, although in smaller quantities (left panel Fig. 5c). Similarly, analysis

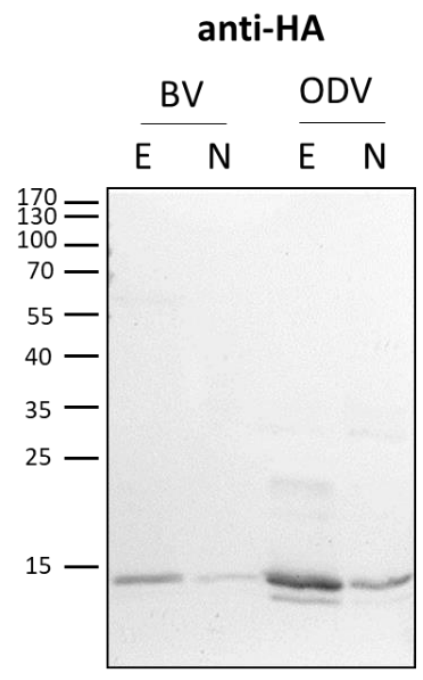

(a)
anti-VP39

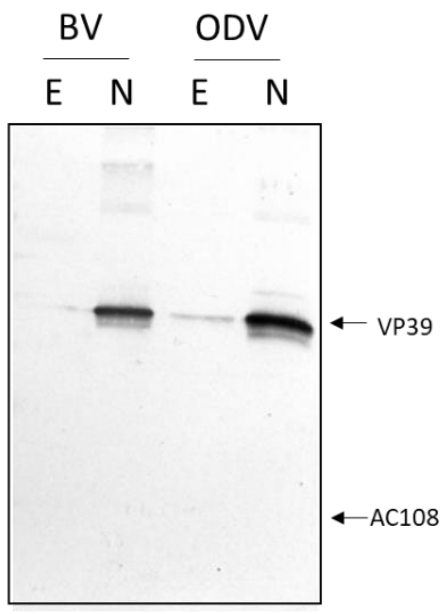

(b)
anti-GP64 anti-PIF1

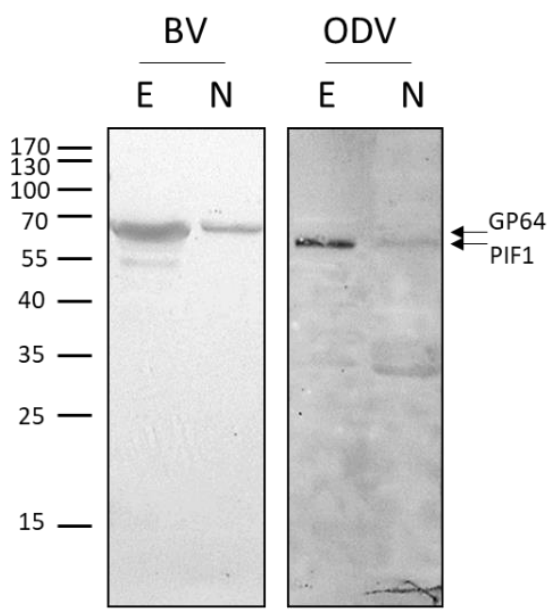

(c)

Figure 5: Western blot analysis of fractionated BVs and ODVs of the HA-tagged ac108 repair mutant. BVs and ODVs were fractionated by incubation in extraction buffer, containing $1 \%$ Triton-X100, in an envelope (E) and nucleocapsid (N) fraction and analysed with (a) anti-HA antibodies to detect AC108, (b) antiserum against VP39 as marker for the nucleocapsids, and (c) antiserum against GP64 as marker for the BV-envelope (left panel), and PIF1 antiserum (right panel) as marker for the ODV envelope. AC108 was detected in BVs and ODVs, in which this protein appeared enriched in the envelope fractions. 
of the ODV fractions with PIF1 antiserum not only resulted in the detection of the $60 \mathrm{kDa}$ protein in the envelope fraction, but small traces were also found in the nucleocapsid fraction (right panel Fig. 5c). Apparently, the BV and ODV envelopes were not completely separated from the nucleocapsid or these proteins interacted with nucleocapsid proteins, resulting in detection of small amounts of envelope proteins in the nucleocapsid fractions. Based on these results, we concluded that $\mathrm{AC} 108$ is a structural component of BVs and ODVs that is mainly associated with the virus envelope.

AC108 is a constituent of the ODV entry complex.

The ODV envelope fractions of the ac108 mutant and the HA-tagged repair viruses were analysed more extensively to determine whether the AC108 protein is involved in formation of the ODV entry complex. Previous research showed that this complex has a stable core, formed by PIF1-4, that resists partial denaturation in Laemmli buffer, when incubated at $50^{\circ} \mathrm{C}$ before gel loading (Peng et al., 2010). Under these conditions, the core complex was detected as a 170 $\mathrm{kDa}$ band by western blot analysis with PIF1 antiserum. When the ODV envelope of the ac108 mutant and repair viruses were analysed under these conditions, the core complex was found at the expected height for both mutants (Fig. 6a). The signal from the complex of the repair mutant was less intense, but the signal from the $60 \mathrm{kDa}$ PIF1 monomers in this lane was also less

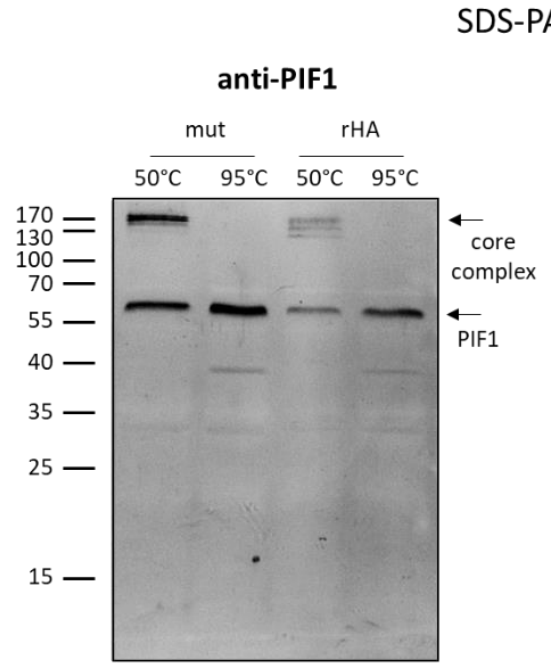

(a)

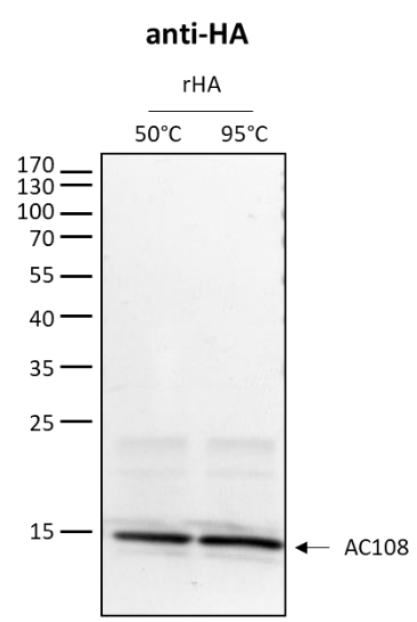

(b)
BN-PAGE

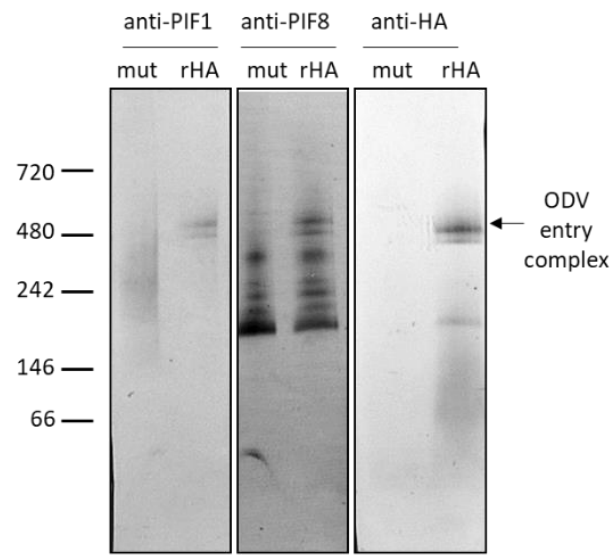

(c)

Figure 6: Western blot analysis of the ODV-envelope fraction of the ac108 mutant (mut) and HA-tagged ac108 repair viruses (rHA) under denaturing and non-denaturing conditions. Under denaturing conditions, the purified proteins were heated at either 50 or $95^{\circ} \mathrm{C}$ and analysed with antiserum against PIF1 (a) or with anti-HA antibodies (b). The ODV envelope proteins were also analysed under non-denaturing conditions with anti-PIF1 antiserum (left panel of c), anti-PIF8 antiserum (middle panel of c) and anti-HA antibodies (right panel of c). 
intense compared to the surrounding lanes, indicating that slightly less protein was loaded. In addition to the $170 \mathrm{kDa}$ complex, smaller complexes (just below $170 \mathrm{kDa}$ ) were also found, especially in the repair virus. Incubation at $95^{\circ} \mathrm{C}$ led to complete dissociation of the core complex as only PIF1 monomers were found. Furthermore, when the repair mutant was analysed with anti-HA antibodies after incubation at $50^{\circ} \mathrm{C}, \mathrm{AC} 108$ was only found in monomeric form with a mass of $15 \mathrm{kDa}($ Fig. $\mathbf{6 b})$. These results indicate that the core complex can still assemble after mutation of the ac108-locus and that the AC108 protein is not a component of the stable core. When the envelope fraction of the HA-tagged repair virus was analysed under non-denaturing conditions by blue native-PAGE followed by western blot analysis, the ODV entry complex was detected as a $480 \mathrm{kDa}$ band, irrespective whether antisera were used against PIF1, PIF8 or HA, indicating that AC108 is a constituent of the ODV entry complex (Fig. 6c). However when analysing the ac108 mutant, the $480 \mathrm{kDa}$ protein complex was not detectable with any of these antisera (and neither was a slightly smaller one due to the absence of AC108), indicating that AC108 is involved in the formation of the entry complex.

\section{Discussion}

The results show that the ac108-gene of AcMNPV encodes a per os infectivity factor, as mutation of this gene abolished ODV oral infectivity, but had no observable effect on the production of BVs and ODVs, nor on the infectivity of BVs. In contrast to all other identified pif-genes, ac108 is currently not considered as a core gene as its homologs have only been detected in viruses classified in the genera Alphabaculovirus, Betabaculovirus and Gammabaculovirus, but not in Culex nigripalpus NPV (CuniNPV), the single known member of the genus Deltabaculovirus (Garavaglia et al., 2012). However, the existence of an ac108 homolog in CuniNPV cannot be entirely excluded though, as the homolog of another pif-gene (ac110, encoding PIF7) was initially missed in CuniNPV as well (Javed et al., 2017). Previous studies on the function of ac108 homologs in SfMNPV and BmNPV resulted in contradictory observations regarding the involvement of their gene products in ODV oral infectivity. In SfMNPV, the ac108-homolog $s f 58$ was shown to be crucial for oral infectivity, while deletion of $b m 91$ in BmNPV increased only the time to death, but did not affect the lethality of the OBs (Simon et al., 2012, Tang et al., 2013). As ac108 is more closely related to bm91 (showing 96\% amino acid sequence identity), it is remarkable to see that its deletion resulted in a different phenotype than observed after deletion of the homologous gene in BmNPV. On the other hand, OB lethality was also affected after deletion of sf58 in SfMNPV (only 36\% amino acid identity with AcMNPV ac108). These conflicting observations might result from the different strategies 
that were used to delete the ac108-homologs, just as observed before with various pif6 mutants (Li et al., 2008, Nie et al., 2012). Another explanation could be that the involvement of AC108 in ODV oral infectivity is species dependent, as shown with the closely related PIF6 homologs in AcMNPV and BmNPV (93\% amino acid identity). In AcMNPV, PIF6 is an ODV-envelope protein that is crucial for oral infectivity, while the homolog in BmNPV, Bm56, is associated with ODV-nucleocapsids and seemed involved in OB-morphogenesis (Nie et al., 2012, Xu et al., 2008). Apparently, two closely related homologous genes do not necessarily have the same function in different viruses, which could also be the case for ac108 homologs. Furthermore, some studies indicate that the proteins which are involved in midgut infection could be different for various virus-host combinations, adding an extra layer of complexity. For example, ac111 in AcMNPV was found to be involved in ODV oral infectivity in Trichoplusia ni larvae, but appeared redundant in S. exigua (Li et al., 2018). Another example is a gene that is only present in group II alphabaculoviruses, hal00 in Helicoverpa armigera NPV (HearMNPV), that also appeared to be involved in ODV oral infectivity (Luo et al., 2011). In light of these findings, it could be reasoned that the ac108-homolog in SfMNPV is apparently more important for ODV oral infectivity in that specific virus-host combination than its homolog in BmNPV. Following this reasoning, it might be interesting to determine whether AC108 is also crucial for ODV oral infectivity in various other permissive hosts other than the S. exigua larvae that were used in this study.

The current study also shows that the AC108 protein is a structural component of ODVs, just as the previously identified PIFs. Furthermore, homologous proteins have been detected in the ODVs of Antheraea pernyi NPV (Shi et al., 2007), BmNPV (Tang et al., 2013), Orgyia pseudotsugata MNPV (Ahrens et al., 1997), SfMNPV (Simon et al., 2012) and Spodoptera litura NPV (Chen et al., 2006). In contrast to the homologues in these other alphabaculoviruses, AC108 was also detected in BVs. However, the absence of AC108 did not result in any observable phenotype regarding the BV infectivity. For PIF4 and PIF6, it was also reported that they were present in ODVs and BVs, and also here without noticeable effects on BV-function after deletion of the corresponding genes (Fang et al., 2009, Nie et al., 2012). So, whether these PIFs have a functional role in BVs, or whether it is a side effect of the routing that newly formed PIFs follow to reach the inner nuclear membrane is still enigmatic.

In previous studies, conflicting results were obtained regarding the interactions of AC108 with other PIFs. In yeast-two hybrid and bimolecular fluorescence complementation assays, AC108 was not found to interact directly with one of the known components of the entry complex 
(Zheng et al., 2017), while this protein was identified as a PIF1 interaction partner in a coimmunoprecipitation study with PIF1 antiserum (Peng et al., 2012). The results of this study are in line with the data obtained from the co-immunoprecipitation study and confirm that AC108 is a component of the ODV entry complex by its detection in the entry complex under non-denaturing conditions. AC108 was not found in a complex with PIF1 under denaturing conditions, indicating that this protein associates with the core complex with relatively low affinity, compared to the interactions between the components of the core complex (PIF1-4). We also show that AC108 is essential for the formation of the ODV entry complex as the complex was not found in the ac108 mutant. PIF4, of the core complex, and the zinc-finger domain of PIF8, a loosely associated entry complex component, have also been shown to be essential for formation of the ODV entry complex (Peng et al., 2012, Javed et al., 2017).

We conclude that ac108 encodes a PIF-protein that mediates ODV oral infectivity in AcMNPV by its more loose association with the ODV entry complex and propose an updated model from Chapter 2, in which AC108, designated as PIF9, is added as a new loosely associated

\section{Baculovirus entry complex in the ODV-envelope}
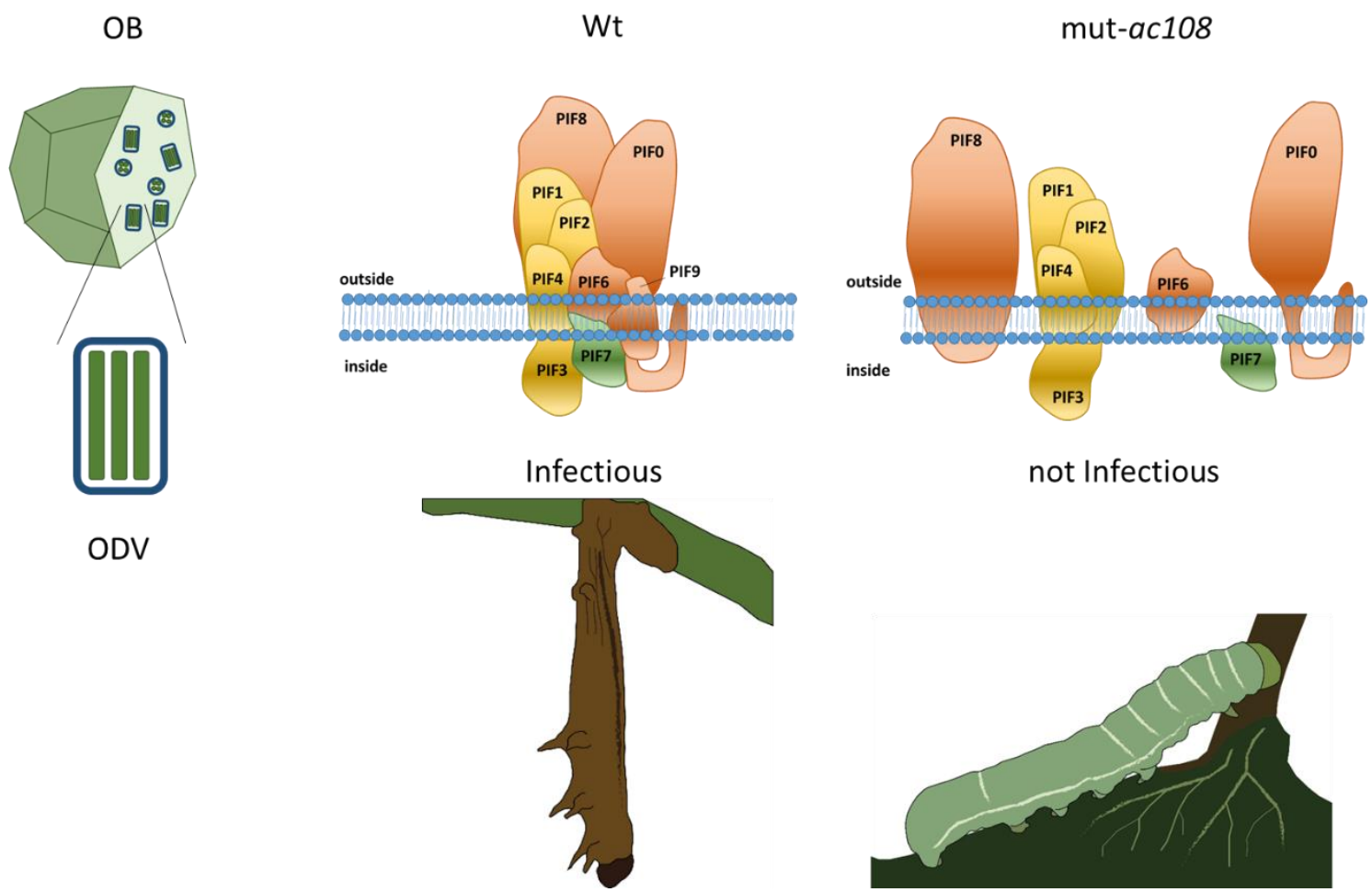

Figure 7: Updated model of the ODV entry complex. Wild type ODVs (Wt) have an intact ODV entry complex in their envelope, containing PIF9, and are orally infectious towards insect larvae (left panel). In absence of PIF9 (mut $a c 108$ ), the stable core is still present but nevertheless fails to form an entry complex, affecting the ODV oral infectivity (right panel). The components of the core complex are depicted in yellow and the loosely associated components are depicted in red. PIF7 is depicted in green as its affinity with the complex is currently not known. 
component (Fig. 7). In absence of PIF9, the stable core can still be formed but not the whole entry complex.

\section{Material and Methods}

Insect cells and lepidopteran larvae. TnH5 cells (from Trichoplusia ni) and Sf21 cells (from Spodoptera frugiperda) were cultured at $27^{\circ} \mathrm{C}$ in Grace's medium (Thermo Fisher), supplemented with $10 \%$ fetal bovine serum (FBS) and $50 \mu \mathrm{g} / \mathrm{ml}$ gentamycin. Spodoptera exigua larvae were reared on artificial diet in a climate room of $25^{\circ} \mathrm{C}$ and $40 \%$ humidity and 16:8 hr (light:dark) photoperiod as described before in Chapter 3.

Construction of recombinant bacmids. The ac108-gene of the AcMNPV bacmid bMON14272 (derived from the E2 strain) was inactivated by insertion of the cat resistance gene via homologous recombination. The cat-gene, flanked by mutated loxP sites (Suzuki et al., 2005), was amplified by PCR with Phusion polymerase (Thermo Fischer) from the pCR-TOPOloxLE-cat-loxRE plasmid (kindly provided by L. Galibert, at the time at Généton, France) with primers that have $50 \mathrm{bp}$ overhangs (in italics), homologous to the sequence that flanks the region to be deleted (5'-GCCGGCGCGACCGCCCGTCGCTCTCGATATAATGTCGGCCGCCGTC GGTTGCTCGGATCCACTAGTAACG-3' and 5'- GCAGCGGCATCACGGTGACGCCCGT CAAGTACAACTATTATTTGTAGTTACCTCTAGATGCATGCTCG -3'). These primers were designed to replace a $12 \mathrm{nt}$ fragment from -8 to +4 relative to the $\mathrm{A}(+1)$ of the start codon of ac108. The amplicon was purified from gel with the GFX Gel Band Purification kit (GE Healthcare) and introduced by electroporation in E. coli MW003 cells (MW001 described in Westenberg et al., 2010 with the bla gene removed), which contained the AcMNPV bacmid. $\lambda$ RED recombination (Datsenko and Wanner, 2000) was activated by heat induction at $42^{\circ} \mathrm{C}$ for 10 min. The transformed bacteria were incubated on LB-agar plates with chloramphenicol (34 $\mu \mathrm{g} / \mathrm{ml})$ and kanamycin $(50 \mu \mathrm{g} / \mathrm{ml})$ for up to two days at $32^{\circ} \mathrm{C}$. Colonies were screened by PCR with primers that annealed outside the recombined region to validate insertion of the cat-gene (5'- CTTGGTTTAACAAGATCACAACC-3' and 5'- CGCGTCTTAACGGACGG-3'). The inserted cat gene was removed by transforming the cells with the temperature sensitive pCRETS plasmid (described in Ros et al., 2015) to allow a CRE-lox reaction that would leave a 100 bp loxP scar. The cells were incubated on LB-agar with kanamycin $(50 \mu \mathrm{g} / \mathrm{ml})$, ampicillin $(100 \mu \mathrm{g} / \mathrm{ml})$ and IPTG $(120 \mu \mathrm{g} / \mathrm{ml})$ at $32^{\circ} \mathrm{C}$. Colonies were transferred to duplicate LB plates, one with kanamycin $(50 \mu \mathrm{g} / \mathrm{ml}$, the other with kanamycin and chloramphenicol $(34 \mu \mathrm{g} / \mathrm{ml})$. The ac108 mutant bacmid was isolated from a colony growing only in absence of chloramphenicol 
and introduced by electroporation into DH1Oß cells that contained the transposase helper plasmid (pMON7124) (Luckow et al., 1993). The polh locus of the ac108 mutant bacmid was restored via Tn7-mediated transposition after electroporation with a modified pFastBac Dual vector, from which the $\mathrm{p} 10$-promoter was removed and the polh ORF was inserted downstream of its native promoter (Peng et al., 2010). The non-mutated AcMNPV bacmid, provided equally with the polh ORF, was used to generate a bacmid-derived wild type virus.

To construct an ac 108 repair bacmid, the putative promotor (from $150 \mathrm{bp}$ upstream of the ATG start codon) and the ac108-ORF were amplified from bMON14272 by PCR with Phusion polymerase (Thermos Fischer) with forward primer 5' - CATCCATGGTTAGTCCG CCCAACACG - 3' and reverse primer 5' - CATGCATGCTTATATTGTTGCATTTCTATT TCTAATATCATAGTTTTC - 3'. For the HA-tagged repair, the reverse complementary sequence of the HA-tag was added to the reverse primer (shown in italics): 5' - ATGCATGC TTAATTAGCGTAATCTGGAACATCGTATGGGTATATTGTTGCATTTCTATTTCTAATA TCATAGTTTTC - 3'. Purified amplicons were digested with NcoI and SphI restriction enzymes (the recognition sites are underlined) and ligated into the corresponding restriction sites of the modified pFastBacDual vector mentioned above. The resulting vectors, containing both polh and ac108 (with and without HA-tag), were used to repair the ac108 mutant bacmid via $\operatorname{Tn} 7$ mediated transposition.

Production of BVs and isolation of OBs. BV-stocks were generated by transfection of Sf21cells with the constructed bacmids, using Expres2TR transfection reagent (Expres2ion Biotechnologies). Six days post transfection, passage 1 (P1) BV-stocks were harvested and amplified by an additional infection round in Sf21 cells to generate high titre P2 BV-stocks. OBs were produced in TnH5 cells, by infecting monolayers of these cells with BVs at an MOI of 2. Five to six days post infection, the cells were harvested and pelleted by centrifugation at $4000 \mathrm{x}$ g for 30 minutes. The cells were then lysed by incubation in $0.1 \%$ SDS for $1 \mathrm{hr}$ at $37^{\circ} \mathrm{C}$ followed by sonication for $1 \mathrm{~min}$ at an output of 8 Watt. Cell lysis was verified by light microscopy and the released OBs were washed twice with Milli Q water. The OBs were further purified by ultracentrifugation over a $30-60 \%(\mathrm{w} / \mathrm{w})$ sucrose gradient in a Beckmann SW32 rotor at $90.000 \mathrm{x} \mathrm{g}$ for $1 \mathrm{hr}$ at $4^{\circ} \mathrm{C}$. The OBs were obtained from the gradient with a Pasteur pipette and pelleted by centrifugation at $4000 \mathrm{x} \mathrm{g}$ for $30 \mathrm{~min}$.

Construction of a BV growth-curve. To determine the BV production of the ac108 mutant and repair viruses, one step growth curves were obtained. For this, Sf21 cells were seeded in a 24- 
wells plate with $3.0 \times 10^{5}$ cells per well and infected in duplo with the various types of BVs at an MOI of 10. The cells were incubated for $1 \mathrm{hr}$ at $27^{\circ} \mathrm{C}$ and washed once with Grace's medium to remove most of the free BVs. Directly after washing $(\mathrm{t}=0)$ and at 24,48 and $72 \mathrm{hpi}$, medium samples were taken and analysed for BV-titres by end-point dilution assays (EPDA).

Electron microscopic analysis of OBs. Purified OBs were dissolved in DAS-buffer on carbon coated copper grids and negatively stained with $2 \%$ phosphotungstic acid, $\mathrm{pH}$ 6.8. The specimens were observed with a JEOL 1400 plus transmission electron microscope.

Bioassays. To determine the oral infectivity of OBs of the ac108 mutant and repair viruses, early L3 S. exigua larvae were starved overnight and then orally inoculated by droplet feeding with $3.0 \times 10^{8} \mathrm{OBs} / \mathrm{ml}$. The OBs were suspended in a $10 \%$ sucrose solution that was coloured blue with Patent Blue V Sodium salt (Fluka). The larvae were allowed to feed on the droplets for $10 \mathrm{~min}$ and the blue coloured larvae were then transferred to 12-wells plates, containing blocks of artificial medium, and incubated at $27^{\circ} \mathrm{C}$. Three days post infection, one day before the expected time to death, the larvae were inspected and non-viral deaths were excluded from further analysis. The larvae were scored for liquefaction until pupation. To test the infectivity of BVs, $1 \mu 1$ normalized BV-stocks $\left(3.5 \times 10^{7}\right.$ TCID $\left._{50} / \mathrm{ml}\right)$ were injected into the hemocoel of L4 S. exigua larvae, using an Humapen Luxura insulin pen. From four days post infection, the larvae were scored on a daily basis for liquefaction.

Protein sample preparation, SDS-PAGE and blue native-PAGE. The ODVs of approximately $3.0 \times 10^{8}$ OBs were released by incubation in DAS-buffer $\left(0.1 \mathrm{M} \mathrm{Na}_{2} \mathrm{CO}_{3}, 166 \mathrm{mM} \mathrm{NaCl}\right.$ and $10 \mathrm{mM}$ EDTA, $\mathrm{pH} 10.5$ ) for $10 \mathrm{~min}$ at $37^{\circ} \mathrm{C}$ and isolated as previously described. The isolated ODVs were fractionated in an envelope- and a nucleocapsid fraction by overnight incubation in extraction buffer $(6.25 \mathrm{mM}$ Tris, $37.5 \mathrm{mM} \mathrm{NaCl}, 1 \%$ Triton X-100, $\mathrm{pH} 7.2)$ at $4^{\circ} \mathrm{C}$. The nucleocapsids were then pelleted by centrifugation at $20.800 \mathrm{x}$ g for $20 \mathrm{~min}$ and the envelope fraction in the supernatant was collected for further analysis. The nucleocapsids in the pellet were resuspended in extraction buffer and also collected for further analysis. The BVs were pelleted by centrifugation at $20.800 \mathrm{x}$ g for $25 \mathrm{~min}$ and fractionated as described above for the ODVs. Both fractions of the BVs and ODVs were analysed by SDS-PAGE and western blot. The protein samples were incubated in Laemmli buffer at either 50 or $95^{\circ} \mathrm{C}$ for $10 \mathrm{~min}$ and separated in a $12 \%$ SDS-PAGE gel. The proteins were transferred to a PVDF membrane under standard conditions. The envelope fractions of ODVs were also analysed under non-denaturing conditions by blue native-PAGE (BN-PAGE), using the NativePAGE ${ }^{\mathrm{TM}}$ Novex $^{\circledR}$ Bis-Tris Gel 
system (Invitrogen), as previously described in Chapter 3. Blotting to a PVDF membrane was in this case performed with NuPAGE® Transfer buffer (Invitrogen) according to the manufacturer's protocol.

Western blot analysis. The blotted PVDF-membranes were analysed with antisera against PIF1 and PIF8 as previously described (Russel and Rohrmann, 1997, Peng et al., 2010, 2012). In brief: rat anti-PIF1 (1:2000 dilution), and rabbit anti-P95 antiserum (PIF8) (1:2000 dilution) were used as primary antibodies. Other membranes were incubated with mice anti-VP39 (1:1000 dilution; kindly provided by Dr R.M. Kotin and Dr L.E. Volkman), and mice anti-GP64 antisera (1:1000 dilution; kindly provided by Dr G.W. Blissard). To detect HA-tagged AC108 in the repair mutant, rat anti-HA antibodies (1:2000 dilution, Roche 3F10) were used as primary antibodies. Goat anti-rabbit (1:2000 dilution, Dako), goat anti-rat (1:2000 dilution, Sigma A8438) and goat anti-mouse (1:2000 dilution, Sigma A5153), conjugated to alkaline phosphatase, were used as secondary antibodies to detect the target proteins by conversion of the NBT-BCIP substrate (Sigma) into a blue-purple precipitant in AP-buffer (0.1 M Tris-HCl, $0.1 \mathrm{M} \mathrm{NaCl}, 5 \mathrm{mM} \mathrm{MgCl}, \mathrm{pH} 10.5)$.

\section{Acknowledgements}

This research was funded by the Netherlands Organization for Scientific Research (NWO), grant number 824.14.16. Felix Evers was involved in this project as a MSc. student and is acknowledged for the construction of the ac108 deletion and repair bacmids and the performance of the initial experiments. I like to thank Els Roode for rearing the insects and culturing the insect cells. 


\section{Supplementary data}

TnH5 cells were infected with $100 \mu 1$ of a P1 BV-stocks to produce OBs at small scale for initial analyses. Six days post infection, the OBs were isolated and counted. The different mutants were found to produce similar amount of OBs as wild type.

\begin{tabular}{cc}
\hline Viruses (bacmid-derived) & Number of OBs \\
\hline Wild type AcMNPV & $1.6 \times 10^{7}$ \\
mut- $a c 108$ & $3.0 \times 10^{7}$ \\
rep-ac108 & $2.56 \times 10^{7}$ \\
rep-acl08HA & $2.67 \times 10^{7}$ \\
\hline
\end{tabular}

This finding was not cell type dependent as similar results were also obtained when OBs were produced in either Sf9 or Sf21 cells by infecting these cells with P2 BV-stocks with an MOI of 2 .

\begin{tabular}{cccc}
\hline $\begin{array}{c}\text { Viruses } \\
\text { (bacmid-derived) }\end{array}$ & Sf9 & Sf21 & TnH5 \\
\hline Wild type AcMNPV & $0.98 \times 10^{8}$ & $0.84 \times 10^{8}$ & $1.9 \times 10^{8}$ \\
mut- $a$ c108 & $0.90 \times 10^{8}$ & $0.42 \times 10^{8}$ & $2.5 \times 10^{8}$ \\
rep- $a c 108 \mathrm{HA}$ & $0.98 \times 10^{8}$ & $0.84 \times 10^{8}$ & $1.9 \times 10^{8}$ \\
\hline
\end{tabular}




\title{
Chapter 5
}

\section{Functional analysis of the baculovirus per os infectivity factors 3 and 9 by imaging the interaction between fluorescently labelled virions and isolated midgut cells}

Bob Boogaard, Jan W. M. van Lent, Monique M. van Oers

\begin{abstract}
The envelope of the baculovirus occlusion derived viruses (ODVs) contains ten different viral proteins, which are classified as per os infectivity factors (PIFs). These proteins are crucial for midgut infection of insect larvae. All these PIFs, with exception of PIF5, are required to form an ODV entry complex. Here, the involvement of PIF3 and 9 in midgut infection is examined in greater detail. PIF3 is known to be dispensable for binding and fusion of ODVs to the brush borders of the epithelial cells, but midgut infection nevertheless fails in absence of PIF3. Where the infection process is halted with the pif 3 deletion mutant virus is currently unknown. PIF9 has only recently been identified and the biological role of this PIF in midgut infection is unknown. Here, the biological role of these PIFs in midgut infection was examined by monitoring the fate of fluorescently labelled ODVs of the Autographa californica multiple nucleopolyhedrovirus, that lack either PIF3 or 9. Isolated midgut cells from Spodoptera exigua larvae were inoculated with the fluorescent mutant ODVs and examined by confocal microscopy. This revealed that in absence of either PIF3 or PIF9, the ODVs bound to the brush borders, but the nucleocapsids failed to enter the cell.
\end{abstract}




\section{Introduction}

The Baculoviridae is a family of arthropod-specific viruses with large circular DNA genomes of 80 - $180 \mathrm{kbp}$, encoding 90 - 180 genes (Williams et al., 2017). This virus family is divided into four genera. The Alphabaculoviruses and Betabaculoviruses infect the larvae of lepidopteran insect species, the Gammabaculoviruses infect hymenopteran larvae and the single known member of the genus Deltabaculovirus infects the larvae of Culex mosquitoes (Jehle et al., 2006). The larvae become orally infected when they consume food sources that are contaminated with occlusion bodies (OBs). These OBs consist of a protein matrix, in which the infectious occlusion derived viruses (ODVs) are embedded. When the OBs are ingested, the protein matrix dissolves in the alkaline environment of the larval midgut lumen and the ODVs are released. These viruses then infect the midgut epithelial cells, in which the ODV-envelope binds and fuses with the plasma membrane of the microvilli of these cells, thus releasing the nucleocapsids into the cells (Kawanishi et al., 1972, Granados et al., 1981). After primary infection of the midgut epithelium, the virus spreads systemically to other tissues by means of a different virus phenotype, the budded viruses (BVs).

Infection of the midgut epithelium cells by ODVs requires at least ten different ODV-envelope proteins: the per os infectivity factors or PIFs (Faulkner et al., 1997, Kikhno et al., 2002, Pijlman et al., 2003, Ohkawa et al., 2005, Fang et al., 2009, Sparks et al., 2011a, Nie et al., 2012, Zhu et al., 2013, Javed et al., 2017, Liu et al., 2016, Wang et al., 2019, Chapter 4). Each of these proteins is essential for infection of the midgut. Deletion of any of the pif-genes abolished the oral infectivity of the ODVs without affecting their formation or embedding into OBs. The production and infectivity of BVs remain unaffected as well. All PIFs, except PIF5, participate in the formation of a large complex, called the ODV entry complex. PIF1 to 4 form the stable core of this complex, which even resists treatment with SDS and ß-mercaptoethanol. PIF0 and PIF6 to 9 dissociate from the complex in the presence of such denaturing chemicals, indicative for a lower binding affinity (Peng et al., 2010, 2012, Wang et al., 2019, Chapter 2, 3, 4). PIF5 apparently promotes ODV infectivity independently from the complex.

Infection of the midgut is initiated by the binding of ODVs to the epithelial cell brush border, followed by fusion of the ODV envelope with the plasma membrane. The nucleocapsids are then released into the cytoplasm, which possibly requires a downstream process that has yet to be characterised (Ohkawa et al., 2005). The initial steps of midgut infection has been studied by fluorescence dequenching assays with R18-loaded ODVs and brush border membrane 
vesicles. This showed that various mutant viruses, lacking either PIF0, 1, 2 or 3, can be phenotypically discriminated in terms of their ability to bind and fuse with prepared brush border vesicles. PIF0, 1 and 2 were shown to be important for the binding of ODVs to the vesicles (Haas-Stapleton et al., 2004, Yao et al., 2004, Ohkawa et al., 2005), while PIF3 was dispensable for ODV binding and fusion (Ohkawa et al., 2005). This indicated that PIF3 is involved in another event during infection of the midgut, which takes place downstream from binding and fusion.

These findings indirectly imply that the binding and fusion of the ODV envelope with the cellular plasma membrane does not require the presence of the entry complex, as the core (and thereby also the entry complex) fails to form in absence of PIF3 (Peng et al., 2010). Furthermore, the entry complex also fails to assemble in absence of PIF9, which just has recently been identified (Chapter 4). However, the role of this new PIF in infection of the midgut remains to be established. To gain more insight in the biological function of PIF3 and PIF9 during midgut infection, we visualized the interaction of fluorescently labelled ODVs, in absence of either PIF3 or 9, with the brush border of isolated midgut cells using confocal microscopy imaging, as previously described by $\mathrm{Mu}$ et al., 2014. This was done with the alphabaculovirus Autographa californica multiple nucleopolyhedrovirus (AcMNPV) and midgut cells isolated from Spodoptera exigua larvae. The current study confirms that ODVs that lack PIF3 are able to bind to the brush borders of midgut epithelial cells, but that the nucleocapsids failed to enter the cells. Similar results were obtained for the pif9 mutant virus.

\section{Results}

Construction of recombinant bacmids to produce fluorescently labelled mutant ODVs that lacks PIF3 or 9 .

The pif3 gene in the AcMNPV bacmid bMON14272 was deleted via insertion of a chloramphenicol acetyltransferase (cat) resistance gene into its open reading frame (ORF) via $\lambda$-red mediated homologous recombination as described before (Datsenko et al., 2000, Li et al., 2007). The cat-amplicon with the homologous arms was produced by PCR by using the same primers as in Li et al., 2007. The insertion of the cat-gene was confirmed by PCR with primers that anneal outside the recombined region. When the wild type bacmid was used as template, a PCR product was produced of 500 bp (Fig. 1). However, a 1500 bp PCR product was produced after homologous recombination, indicating that the cat-gene was successfully inserted into the 
PCR validation of the insertion of a chloramphenicol resistance (cat) gene into the pif3-ORF

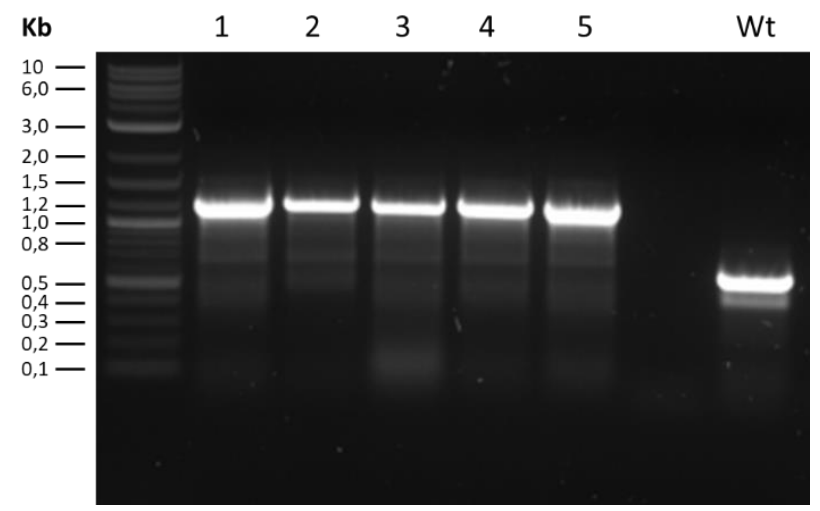

Figure 1: Confirmation of the insertion of the chloramphenicol acetyl transferase (cat) resistance gene into the pif3 locus by PCR. Insertion of the cat-gene resulted in the formation of an amplicon 1500 bp (clone 1-5), while without insertion a $500 \mathrm{bp}$ amplicon was produced $(\mathrm{Wt})$.

ORF of pif3. The cat-gene was found inserted in all five clones and clone 1 was used for further studies. The pif3 deletion bacmid was used for site directed transposition using a modified pFastBac Dual vector that contains the polyhedrin (polh) gene under the polyhedrin promoter, and the ORF of $v p 39$ fused to the 3 'end of the egfp-ORF, expressed under the p10 promoter (pFastBac-polh-egfp:vp39) (Fig. 2). An ac108 mutant bacmid, previously described in Chapter 4, was also transposed with this pFastBac-polh-egfp:vp39 vector to obtain mut-pif9 (Fig. 2). In this way, the resulting del-pif3 and mut-pif9 viruses are able to produce OBs and fluorescent

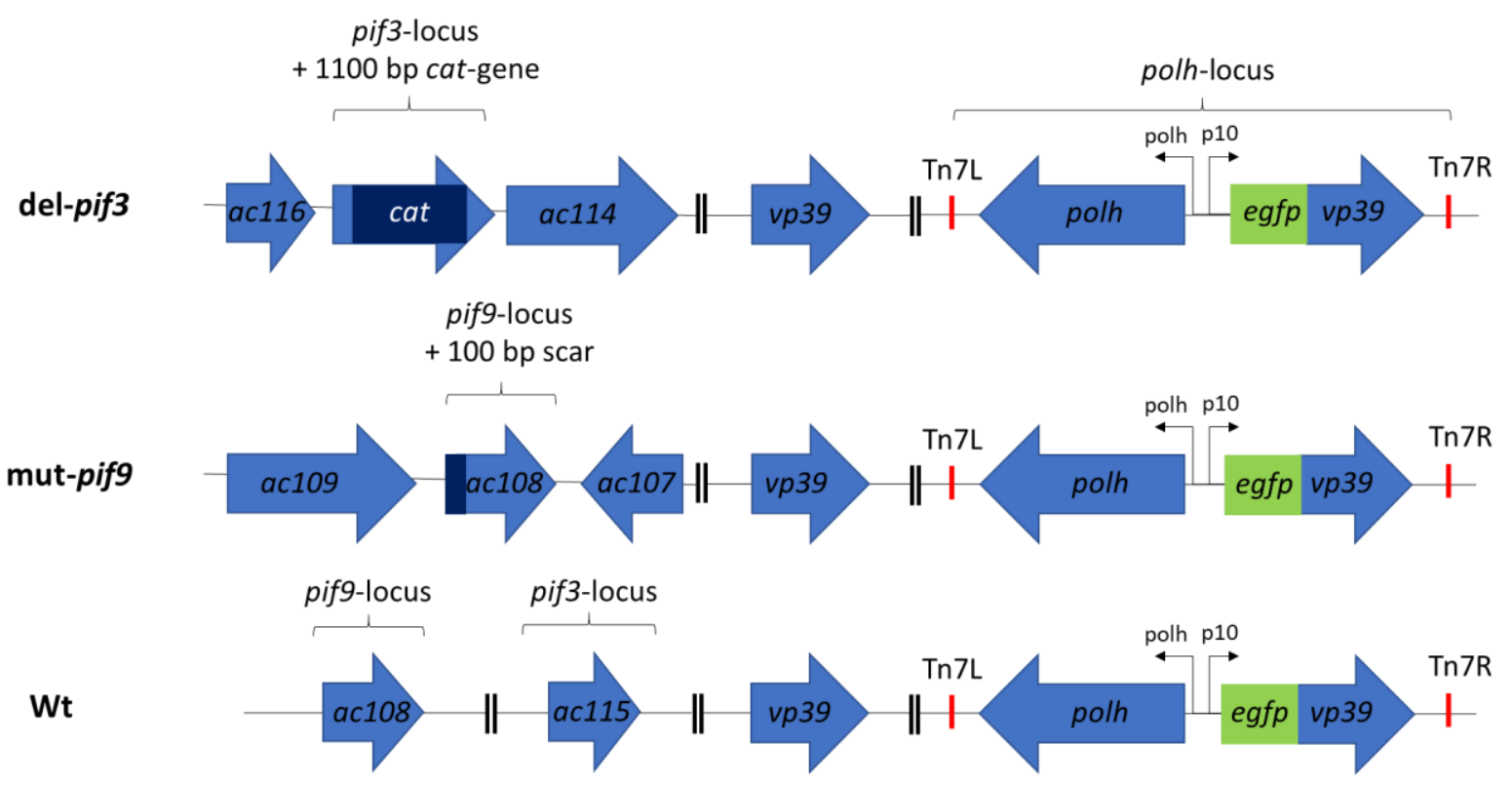

Figure 2: Overview of the constructed bacmids. The pif3 deletion and pif9 mutant bacmids (the latter is described in Chapter 4) were transposed with a pFastbac-polh-egfp:vp39 vector to enable the mutant viruses to produce OBs and fluorescently labelled nucleocapsids. The fluorescent wild type virus was previously described in $\mathrm{Mu}$ et al., 2014 and is used in this study as wild type control. 
nucleocapsids. The fluorescent "wild type" (Wt) virus used in this study was previously described in Mu et al., 2014.

\section{Western blot analysis of the mutant ODVs envelope and nucleocapsid fractions}

To confirm the absence of PIF3 in the ODV envelope after pif3 deletion, as well as the incorporation of the eGFP:VP39 fusion protein into the nucleocapsids, the ODVs of the wt and mutant viruses were fractionated and analysed by western blotting. The envelope fractions were analysed with PIF3 antiserum and the nucleocapsid fractions with antiserum against the major capsid protein VP39. These analyses showed that PIF3 monomers were found at the expected height (corresponding to $27 \mathrm{kDa}$ ) in the envelope fractions of the wt and mut-pif9 virus, but not in the del-pif3 virus (Fig. 3a). When the nucleocapsid fractions of these three viruses were analysed with antiserum against VP39, the capsid protein was detected as a $39 \mathrm{kDa}$ protein and the eGFP:VP39 fusion protein was detected just below the $70 \mathrm{kDa}$ marker, as expected, although in smaller quantities than the native VP39 protein (Fig. 3b). This indicated that the fusion protein was incorporated into the nucleocapsids of the wt and mutant viruses.

\section{The infectivity of the fluorescent viruses for S. exigua larvae}

The infectivity of the fluorescent viruses was tested in S. exigua L4 larvae via intra-hemocoelic injection of BVs and by oral inoculation of early L3 larvae with OBs. The oral infectivity of the pif3 deletion and pif9 mutant ODVs were determined in separate bioassays, in which the

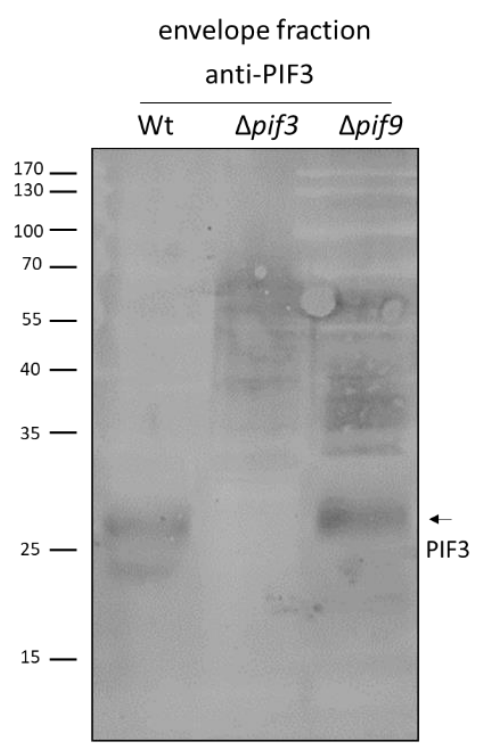

(a)

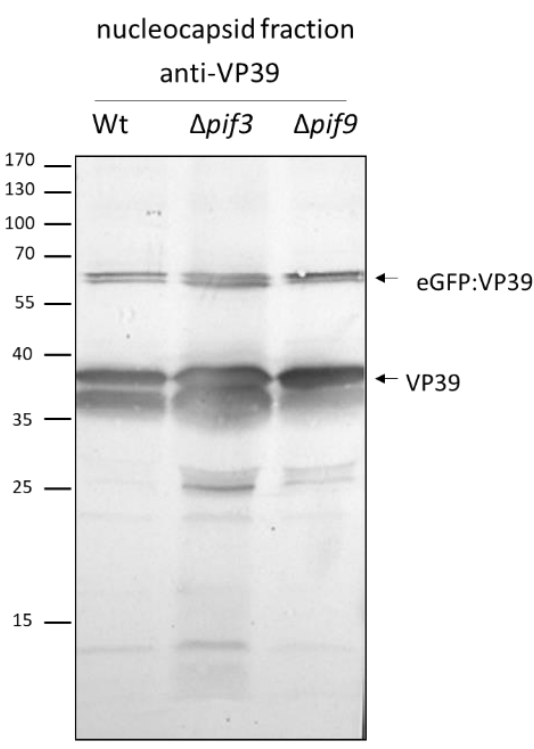

(b)

Figure 3: Western blot analysis of the ODV-envelope and nucleocapsid fractions of wild type, pif3 deletion mutant and pif9 mutant viruses. The envelope fractions were analysed with antiserum against PIF3 (a) and the nucleocapsid fractions with VP39 antiserum (b). 
Table 1: Mortality of S. exigua larvae after injection with BVs or oral inoculation with OBs

\begin{tabular}{|c|c|c|c|}
\hline Injection with BVs & \multicolumn{3}{|c|}{$3.5 \times 10^{7} \mathrm{TCID}_{50} / \mathrm{ml}$} \\
\hline mock & \multicolumn{3}{|c|}{$0 / 12$} \\
\hline del-pif3-egfp:vp39 & \multicolumn{3}{|c|}{$11 / 12$} \\
\hline mut-pif9-egfp:vp39 & \multicolumn{3}{|c|}{$11 / 12$} \\
\hline Wt-egfp:vp39 & \multicolumn{3}{|c|}{$12 / 12$} \\
\hline Oral infectivity $3.0 \times 10^{8} \mathrm{OBs} / \mathrm{ml}$ & \multicolumn{2}{|r|}{ Exp. 1} & Exp. 2 \\
\hline mock & \multicolumn{2}{|r|}{$0 / 14$} & $0 / 12$ \\
\hline del-pif3-egfp:vp39 & \multicolumn{2}{|r|}{$0 / 13$} & $0 / 11$ \\
\hline Wt-egfp:vp39 & \multicolumn{2}{|r|}{$12 / 13$} & $11 / 11$ \\
\hline Oral infectivity $3.0 \times 10^{8} \mathrm{OBs} / \mathrm{ml}$ & Exp. 1 & Exp.2 & Exp. 3 \\
\hline mock & $0 / 12$ & $0 / 14$ & $0 / 12$ \\
\hline mut-pif9-egfp:vp39 & $0 / 15$ & $0 / 12$ & $0 / 12$ \\
\hline Wt-egfp:vp39 & $12 / 15$ & $12 / 13$ & $10 / 12$ \\
\hline
\end{tabular}

pif3 deletion mutant was tested in duplicate and the pif9 mutant in triplicate, because additional information is available on the function of PIF3 in literature, whereas PIF9 just has been identified (Table 1). When the larvae were injected with Wt or one of the mutant BVs stocks, fluorescence was observed in at least 11 out of 12 larvae for each virus at $3 \mathrm{dpi}$, in contrast to the mock injected larvae (Fig. 4a). This indicates that the eGFP:VP39 fusion protein is produced and that the Wt and pif mutant viruses replicate in the larvae when bypassing the midgut. When the larvae were orally inoculated with OBs, only the larvae that were fed with the wt virus showed fluorescence (Fig. $\mathbf{4 b}$ ). These observations correspond with the mortality rates, in which ODV inoculation only caused significant mortality for the Wt virus (Table 1). These results show that the fluorescently labelled pif mutant viruses have the same phenotype as previously described for the non-fluorescent mutant viruses (Li et al., 2007, Boogaard et al., 2019).

Wild type ODVs enter the midgut cell within $2-6$ minutes after the start of observation.

To visualize the interaction between (mutant) ODVs and the epithelial cell brush border, isolated midgut cells of S. exigua larvae were incubated with fluorescently labelled ODVs and examined by confocal microscopy to monitor the location of the ODVs in relation to time. These analyses were first performed for Wt ODVs to validate whether cell entry can be observed under the applied experimental conditions. Twenty one Wt ODVs were followed over 


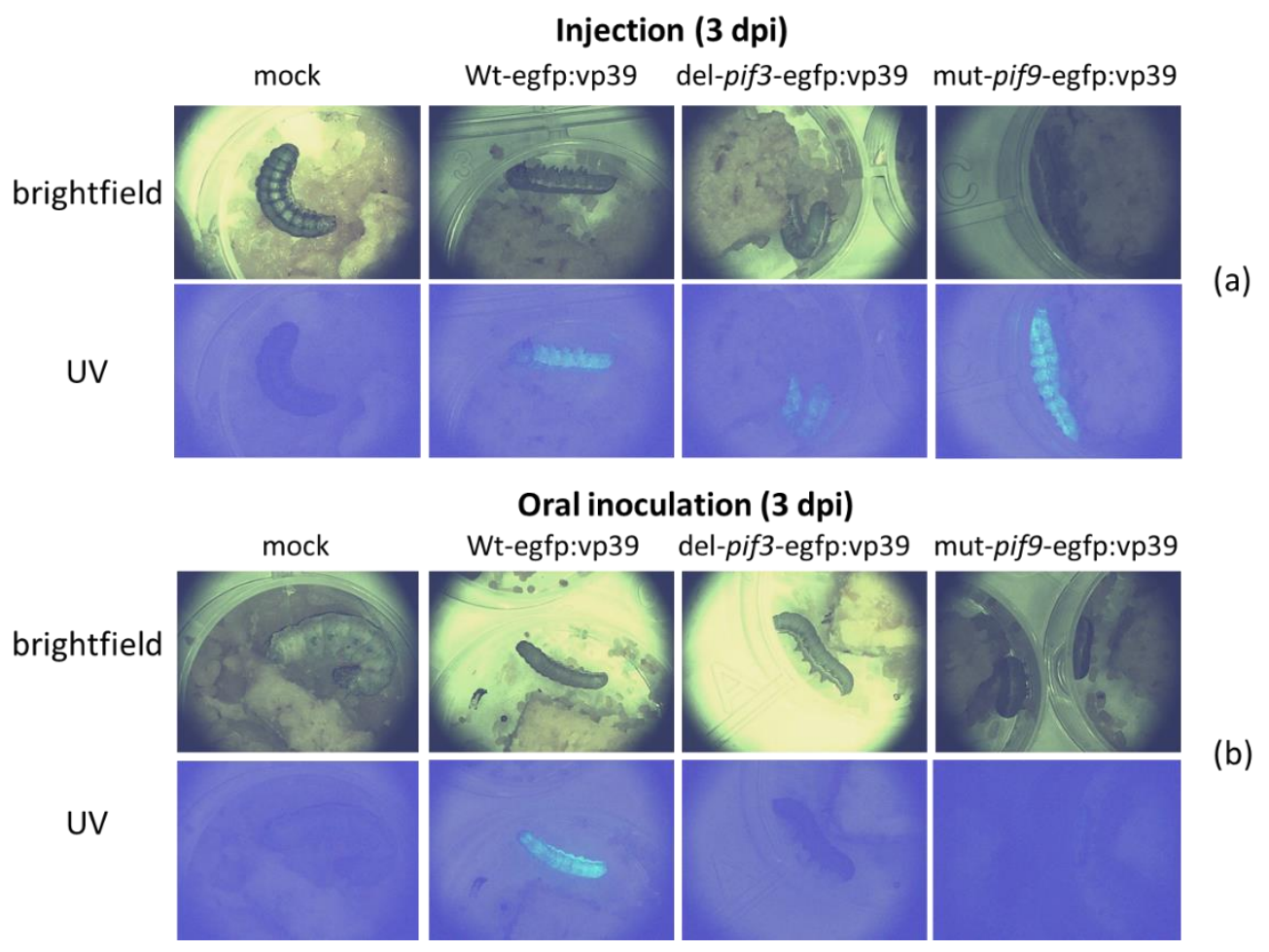

Figure 4: Validation of viral replication of eGFP-tagged AcMNPV mutants in S. exigua larvae. The larvae were examined under UV-light to observe green fluorescence after injection with BVs (a) or oral inoculation with OBs (b). Green fluorescence is indicative for viral replication.

time and these interacted with eight separate midgut cells (Table 2). These ODVs were detected in the brush border for 2 to 6 minutes before they disappeared from the cell surface (Fig. 5). About half of the ODVs disappeared within 2-4 minutes after the start of the time lapse experiment. As the ODVs under study could also not be found in the surrounding focal planes, it was concluded that the nucleocapsids had entered the midgut cell.

Table 2: Overview of the observations of fluorescent ODVs interacting with the brush border of primary midgut cells.

\begin{tabular}{cccc}
\hline Virus & $\begin{array}{c}\text { Nr. of } \\
\text { observations }\end{array}$ & $\begin{array}{c}\text { Nr. of observed } \\
\text { cells }\end{array}$ & $\begin{array}{c}\text { Average observation time before } \\
\text { disappearance (min.) }\end{array}$ \\
\hline Wild type & 21 & 8 & $4.5 \pm 2$ \\
del-pif3 & 7 & 4 & - \\
mut-pif9 & 14 & 4 & - \\
\hline
\end{tabular}

Mutant ODVs that lack either PIF3 or 9 bind to the brush border, but fail to enter the cell.

The experiment was repeated with mutant ODVs, for which seven ODVs of the pif 3 deletion mutant and fourteen of the pif9 mutant were analysed, each on four distinct midgut cells (Table 2). The ODVs of both mutants were detected at the cell surface until the end of the 


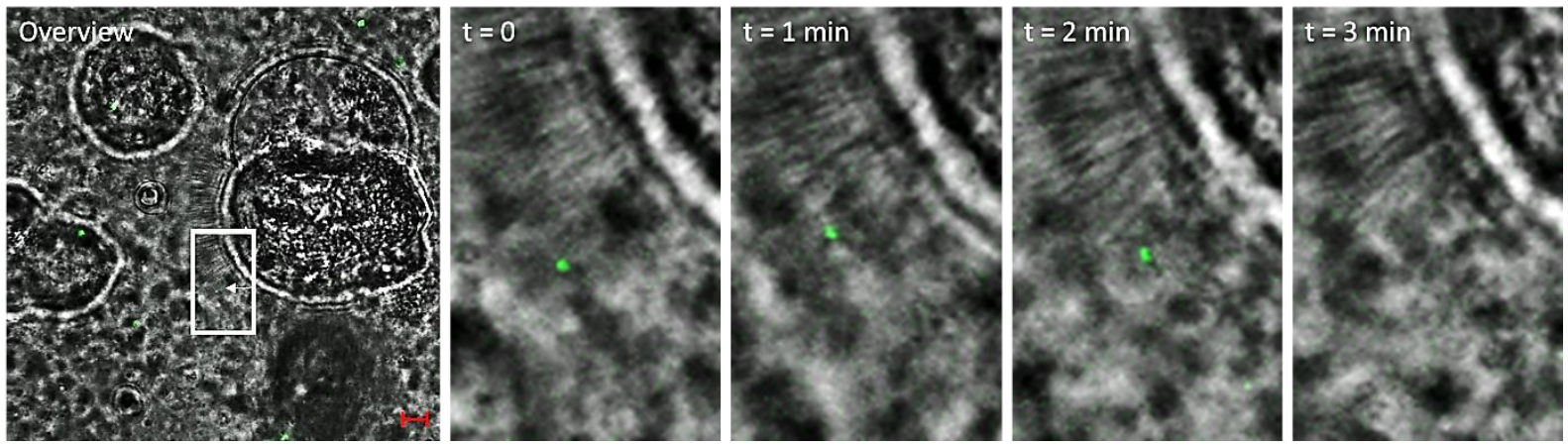

Figure 5: Time lapse analysis of one fluorescently labelled wild type ODV on the brush border of an isolated midgut cell. The red scale bar corresponds with $5 \mu \mathrm{m}$. In this case, the ODV disappeared within 3 minutes after the start of the observation.

measurements, even after continuous excitation with the laser for 20 minutes of the eGFP:VP39 fusion protein (Fig. 6). These observations show that in absence of PIF3 or PIF9 the ODVs are still able to bind to the brush borders of midgut epithelial cells, but the nucleocapsids fail to enter the cell.

\section{Discussion}

Baculovirus ODVs infect the midgut epithelium of insect larvae via direct membrane fusion with the plasma membrane of the host cell brush border (Kawanishi et al., 1972, Horton et al., 1993). After the ODV envelope has fused with the plasma membrane, the nucleocapsids enter the cell and are transported through the microvilli and the rest of the cytoplasm to the nucleus for replication (Kawanashi et al., 1972, Tanada et al., 1975, Granados et al., 1981). The ODVenvelope contains at least ten different proteins that are crucial for midgut infection, PIF0 to 9, of which nine (all except PIF5) form the ODV entry complex (Peng et al., 2010, 2012, Chapter $\mathbf{2}, \mathbf{3}, \mathbf{4})$. The formation of this complex is abolished when one of these components is absent.

Although the complex cannot be formed in absence of one of the PIFs (Peng et al., 2010, 2012, Chapter 4), two different phenotypes regarding binding and fusion have been observed with R18 dequenching assays when using various mutant ODVs. It had been found that PIF0, 1 and 2 are important for ODV binding (Haas-Stapleton et al., 2004, Yao et al., 2004), while PIF3 is dispensable for binding and fusion (Ohkawa et al., 2005). The ODVs of the pif3 deletion mutant nevertheless failed to establish an midgut infection. PIF9 has been identified only recently (Chapter 4). However, no data on the biological role of this protein has been reported. This study aims to complement the existing knowledge on the function of PIF3 in midgut infection and to obtain the first data on the biological function of PIF9. Therefore, fluorescently labelled ODVs of AcMNPV that lack either PIF3 or 9 were generated and followed over time in the 


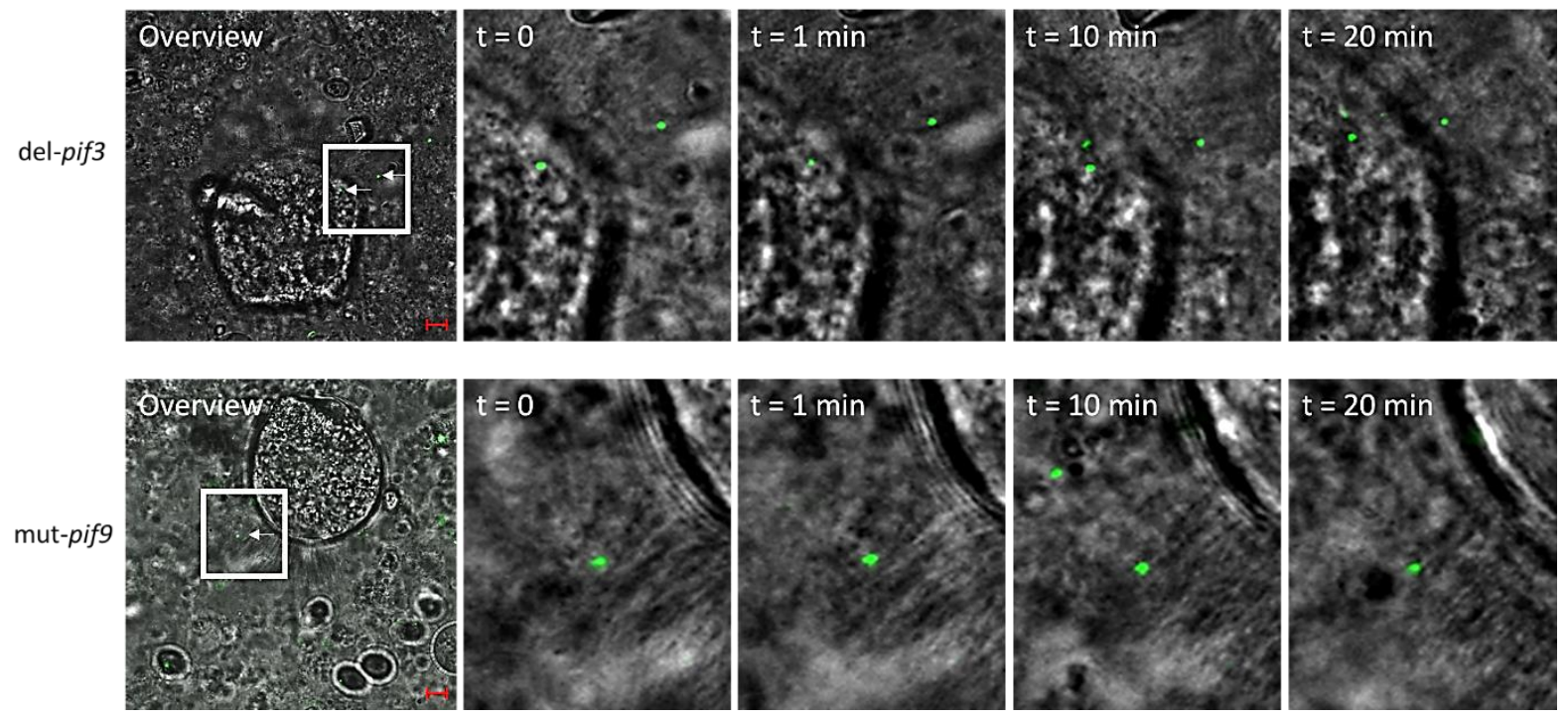

Figure 6: Time lapse analysis of one (representative) fluorescently labelled ODV of a pif3 deletion mutant and a pif 9 mutant virus on the brush border of an isolated midgut cell. The red scale bar corresponds with 5 $\mu \mathrm{m}$. The fluorescent mutant ODVs remain attached to the brush borders to the end of the observations.

brush borders of isolated midgut cells from $S$. exigua larvae with a confocal microscope, analogous to the assay that had been described in $\mathrm{Mu}$ et al., 2014.

Wild type ODVs were detected on the brush border for 2 to 6 minutes before they disappeared from the cell surface, while in absence of PIF3 or 9 the ODVs remain detectable until the end of the measurements (Fig. 5-6). Hence, the disappearance of wild type ODVs could not be explained by fading of the fluorescent signal by continuous excitation of the eGFP:VP39 fusion protein with the laser. As the wild type ODVs were also not found in the flanking sections, it was concluded that the fluorescent signal disappeared by decomposition of the grouped nucleocapsids of the multiple capsid ODV upon cell entry, as was observed before in electron microscopy studies (Kawanashi et al., 1972, Granados et al., 1981). It took longer for the Wt ODVs to enter the cells under the present experimental conditions than previously described in Mu et al., 2014. This might be explained by the usage of ODVs that were released from cell culture-derived OBs (C-ODVs) instead of OBs from infected larvae (L-ODVs), as used in the other study. Infection with C-ODVs might initially be hampered by remnant polyhedrin that sticks to the virus envelope, which is degraded by co-occluded host proteases in L-ODV preparations (Harrap et al., 1977, Payne and Kalmakoff, 1977, Wood et al., 1980). However, as the remaining PIF-proteins in pif mutants are highly sensitive to degradation by such cooccluded proteases during OB-dissolution (Chapter 2), we decided to use cell-culture derived viruses for the experiments. The remnant polyhedrin on the C-ODVs was probably degraded by (membrane associated) digestive enzymes of the primary midgut cell (Javed et al., 2019) when the virus and cell suspensions were mixed, enabling the ODVs to enter the cell after a 
few minutes. Whether the PIFs in the mutant viruses underwent a certain level of degradation under the conditions used was not determined. The proteolytic activity cannot be completely inhibited, e.g. by adding a cocktail of protease inhibitors, as this hampers the infection by ODVs (Slack et al., 2008). PIF0 has been shown to be the cleaved by host trypsins in the gut and by co-occluded proteases (Slack et al., 2008, Peng et al., 2011), but the importance of these cleavage events for ODV infectivity needs further studies.

Our observations show that in absence of PIF3, the ODVs bind to the brush border (Fig. 6), just as indicated before by the R18 dequenching assays (Ohkawa et al., 2005). Whether the ODVenvelope of this mutant also fused with the brush border plasma membrane could not be confirmed with the currently used assay. Our observations nevertheless show that the nucleocapsids stayed together and failed to enter the cell. This indicates that PIF3 is indeed required for the translocation of the nucleocapsids into the host cell as proposed by others (Ohkawa et al., 2005). However, it has been observed with mutants of other viruses that R18dequenching also takes place after hemifusion of the lipid membranes, in which only the outer leaflets of the bilayers fuse (Kemble et al., 1994, Laliberte et al., 2011). It could therefore also be reasoned that in absence of PIF3, only the outer leaflets fuse and that this PIF is required to overcome this hemifusion intermediate state (Fig. 7). This might explain why the R18dequenching assays with the pif3 deletion mutant suggested that membrane fusion took place, while the infection of the midgut epithelium failed.

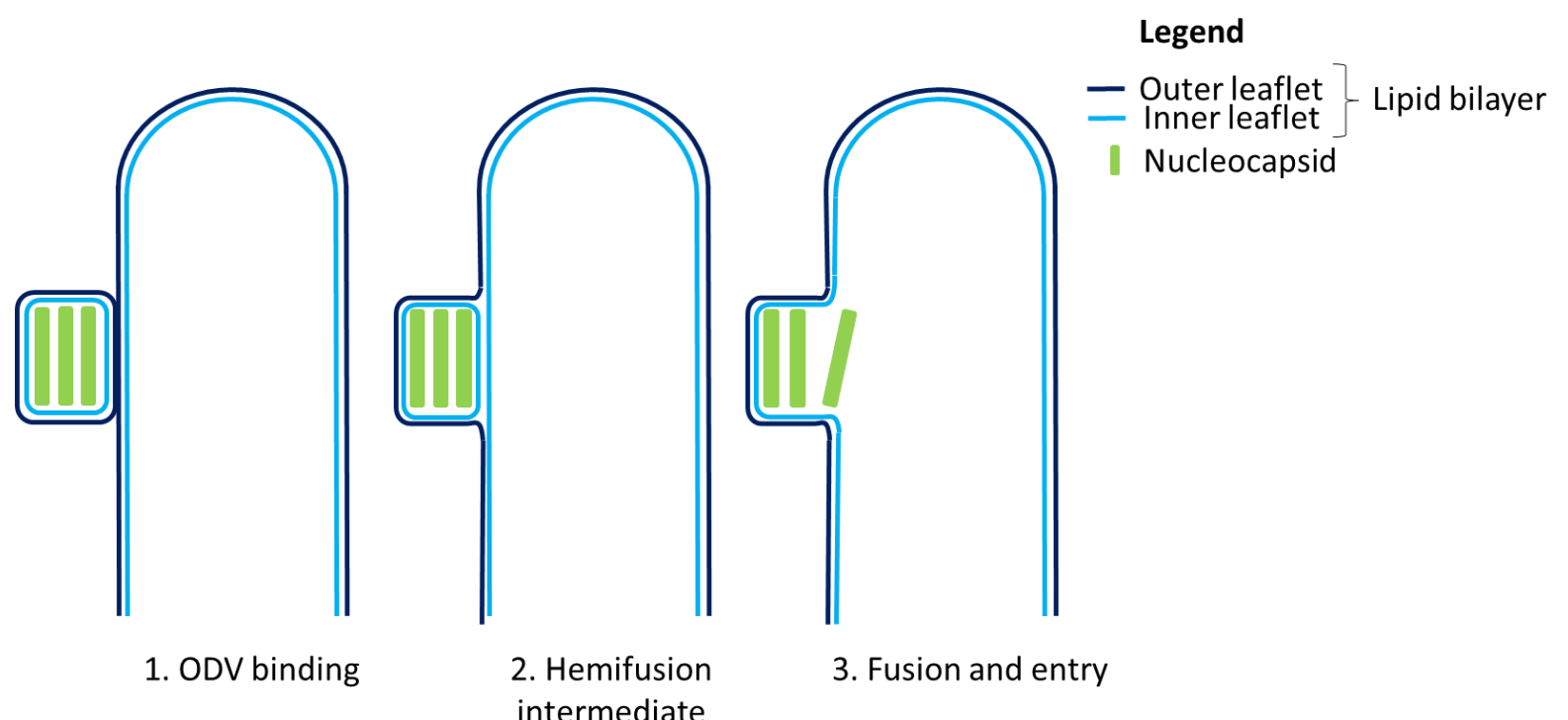

Figure 7: Model for the membrane fusion event. During primary infection of the midgut epithelium, the ODV first binds to the microvilli (1) and membrane fusion occurs via the formation of a hemifusion intermediate (2). It is hypothesized that in absence of PIF3, membrane fusion is stuck in this intermediate state and prevents entry of the nucleocapsids into the host cell. 
ODVs that lack PIF9 also bound to the epithelial cell brush borders. Whether the binding of these mutant ODVs is indeed not affected needs to be validated quantitatively by R18 dequenching assays, as the live imaging assay is biased for ODVs that bind to the brush borders; i.e. non-interacting ODVs were not analysed in the time lapse experiments. However, as we regularly encountered bound ODVs, it is reasoned that binding is not significantly affected by the absence of PIF9. Whether this PIF is involved in membrane fusion needs also to be determined by R18-dequenching assays. Our observations nevertheless show that midgut entry of the nucleocapsids failed in absence of PIF9, as shown previously for PIF1, 2 (Mu et al., 2014), and now also for PIF3.

Despite the fact that both mutants are unable to form the ODV entry complex, the mutant ODVs were nevertheless found to be able bind to the brush border of the isolated midgut cells. Formation of the entry complex is apparently not important for the ODV binding properties as long as PIF0, 1 and 2 are present in the ODV envelope. It was also observed for both mutants that the entry of the nucleocapsids was abolished. The exact reason for this is unfortunately unclear. It could be concluded from these observations that formation of the entry complex is crucial for the early stages of midgut infection, either for completion of membrane fusion at the epithelial cell brush border or for the translocation of the nucleocapsids into the cytosol.

\section{Materials and methods}

Insect cells and lepidopteran larvae. TnH5 (from Trichoplusia ni) and Sf21 cells (from Spodoptera frugiperda) were cultured in Grace's medium (Thermo Fisher), supplemented with $10 \%$ fetal bovine serum (FBS) and $50 \mu \mathrm{g} / \mathrm{ml}$ gentamycin (Gibco, Life technologies) at $27^{\circ} \mathrm{C}$. Spodoptera exigua larvae were reared on artificial diet in a climate room of $25^{\circ} \mathrm{C}$ and $40 \%$ humidity and 16:8 hr (light:dark) photoperiod as described before (Chapter 3).

Construction of recombinant bacmids. The ORF of pif 3 of the AcMNPV bacmid bMON14272 (derived from the E2 strain) (Luckow et al., 1993, Smith and Summers, 1978) was deleted by insertion of the chloramphenicol acetyl transferase (cat) resistance gene via homologous recombination, as described in Li et al., 2007. The cat-gene was amplified by PCR with Phusion polymerase (Thermo Fischer) from the pCR-TOPO-loxLE-cat-loxRE plasmid (kindly provided by L. Galibert, at the time at Généton, France) with primers that have 50 bp overhangs (in italics), homologous to the sequence in the pif3-ORF that flanks the sequence that needs to be deleted. (5-CACGGGGATCGTGCGAGCCAGTGCCGTTTGGCGAAATAGCATTTTTTTCAT GCTCGGATCCACTAGTAACG -3' and (5'-TACACGTTTAAATTTGTTCAAAAATTTATTTT 
GCAAGATGCATACATGCACCTCTAGATGCATGCTCG -3'). The amplicon was purified from an agarose gel with the GFX Gel Band Purification kit (GE Healthcare) and electroporated into E. coli MW003 cells (MW001 (Westenberg et al., 2010) with the bla gene removed), carrying the AcMNPV bacmid. The expression of the $\lambda$ RED for recombination (Datsenko and Wanner, 2000) was activated in these bacterial cells by heat induction at $42^{\circ} \mathrm{C}$ for $10 \mathrm{~min}$. The transformed bacteria were incubated on LB-agar plates in the presence of chloramphenicol (34 $\mu \mathrm{g} / \mathrm{ml})$ and kanamycin $(50 \mu \mathrm{g} / \mathrm{ml})$ for up to two days at $32^{\circ} \mathrm{C}$. Colonies were screened by PCR with primers that annealed outside the recombined region to validate insertion of the cat-gene into the pif3-ORF (5'-CAGTTGTAAACGCGTCTGTAC -3' and 5'- CATGTTGAAC TTTTGGCAAATACTTATTC-3'). The pif3 deletion bacmid was isolated, by alkaline lysis of the bacterial cells and subsequent precipitation in isopropanol, and electroporated into DH10ß cells that contained the transposase helper plasmid (pMON7124) (Luckow et al., 1993). Construction of the pif9 mutant bacmid is described in Boogaard et al., 2019. Both bacmids were transposed with a pFastBac Dual vector of the Bac-to-Bac system with the polh-gene under the polh-promoter and the ORF of egfp fused to the 5'end of the vp39-ORF under control of the p10 promoter (pFastbac-polh-egfp:vp39) as described in Mu et al., 2014. These genes were transposed via Tn7 mediated transposition into the polh-locus of the bacmids to enable the resulting viruses to produce OBs and fluorescently labelled nucleocapsids.

Production of the mutant viruses. First generation BV-stocks (P1) of the pif3 and pif9 mutants were generated by transfection of Sf21 cells with the bacmids, using Expres2TR transfection reagent (Expres2ion Biotechnologies). Six days post transfection, the BV stocks were amplified by an additional infection round in Sf21 cells, generating P2 BV-stocks. OBs were produced by infection of TnH5 cells with BVs at an MOI of 2 and isolated as described before (Chapter 4). In brief, the infected cells were harvested at six days post infection by centrifugation at $4000 \mathrm{x} \mathrm{g}$ for $30 \mathrm{~min}$ and lysed by incubation in $0.1 \%$ SDS for $2 \mathrm{hrs}$. at $37^{\circ} \mathrm{C}$ with gentle agitation and subsequent sonication for 1 minute at an intensity of 8 Watt on ice. Complete lysis was verified by light microscopy and the cells were sonicated once more when needed. When all the cells had lysed, the OBs were pelleted by centrifugation at $4000 \mathrm{x} g$ for 30 min. and washed three times with sterile double distilled water.

SDS-PAGE and western blot analysis. The ODVs of the pif3 and pif9 mutants were isolated, fractionated and analysed by SDS-PAGE and western blotting as described previously in Chapter 3, 4. The envelope fraction was analysed with rat-derived PIF3 antiserum (described in Peng et al., 2010, diluted 1:1000) and the nucleocapsid fraction with antiserum against the 
major capsid protein VP39, derived from mice (diluted 1:1000). Goat anti-rat (1:2000, Sigma A8438) and goat anti-mouse (1:2000, Sigma A5153), conjugated to alkaline phosphatase, were used as secondary antibodies to detect the target proteins via the conversion of the NBT-BCIP substrate (Roche) in a purple precipitant in AP-buffer (0.1 M Tris- $\mathrm{HCl}, 0.1 \mathrm{M} \mathrm{NaCl}$ and $5 \mathrm{mM}$ $\mathrm{MgCl}, \mathrm{pH} 10.5)$.

Bioassays in S. exigua larvae. The oral infectivity of the OBs of the fluorescent pif3 and pif9 mutant viruses was tested by oral inoculation of starved early L3 S. exigua larvae in droplet feeding assays. Fluorescent wt virus (previously described in Mu et al., 2014) was used as positive control. The OBs were suspended in $10 \%(\mathrm{w} / \mathrm{v})$ sucrose at a concentration of $3.0 \times 10^{8}$ OBs / $\mathrm{ml}$ and the suspension was coloured blue with Patent Blue V Sodium salt (Fluka). The larvae were allowed to feed on the OB-sucrose solutions for 10 minutes, transferred to 12 -wells plates with artificial medium in each well and incubated at $27^{\circ} \mathrm{C}$. One day prior to the expected time of death ( $3 \mathrm{dpi}$ ), the larvae were scored for green fluorescence under UV light (indicative for viral replication) and incidental non-viral deaths were excluded from further analyses. The infectivity of the BVs of the fluorescent pif3 and pif9 mutant viruses was determined by injection of $1 \mu \mathrm{BV}$-stock $\left(3,5 \times 10^{7} \mathrm{TCID}_{50} / \mathrm{ml}\right)$ into the hemocoel of L4 larvae, using an Humapen Luxura insulin pen. These larvae were also scored for green fluorescence at 3 dpi as described above. For both assays, the number of liquefied larvae were scored on a daily basis.

Isolation of the midgut cells from S. exigua larvae. The midguts of twenty S. exigua L5 instar larvae were dissected by cutting of the larva's head and tail, after which the midgut was pulled out and the peritrophic membrane was removed. The midguts were collected in Graces medium, supplemented with $50 \mu \mathrm{g} / \mathrm{ml}$ gentamycin, washed twice (also in Graces medium) and incubated in Graces medium, containing $2 \mathrm{mg} / \mathrm{ml}$ collagenase from Clostridium histolyticum (Sigma, blend type $\mathrm{H}$ ), for $90 \mathrm{~min}$. at $27^{\circ} \mathrm{C}$ under vigorous shaking at $850 \mathrm{rpm}$. The cells were separated from the remaining midgut tissues by filtering through a $200 \mu \mathrm{m}$ meshwork of nylon, fixed at the bottom of a microtube. The primary midgut cells were collected and pelleted by centrifugation at $200 \mathrm{x} \mathrm{g}$ for $10 \mathrm{~min}$, and subsequently washed three times with Grace's medium. After the last washing step, the cells were suspended in pre-cooled EDTA-free DAS buffer $\left(0.1 \mathrm{M} \mathrm{Na}_{2} \mathrm{CO}_{3}, 166 \mathrm{mM} \mathrm{NaCl}, \mathrm{pH} 10.5\right)$ and kept on ice till further use.

Time-lapse analysis of fluorescent ODVs on the brush border of primary midgut cells. The interaction between the fluorescent ODVs and the brush borders of the isolated midgut cells was visualized by constructing a z-stack in a time lapse experiment with a confocal microscope 
(Zeiss LSM-510). The ODVs were isolated in slightly a modified way from what was described in Chapter 3. The ODVs were released by a 10 min incubation in EDTA-free DAS-buffer and the OB membrane was disrupted by two sonication bursts. The ODVs were incubated with the isolated midgut cells under alkaline conditions in EDTA-free DAS-buffer on ice for 15 min to allow these to bind to the brush borders prior to imaging. The cell-virus suspension was examined for midgut cells with (bound) ODVs in the brush border by detection of GFP fluorescence by confocal microscopy, using a 63x oil objective lens under excitation with an Argon laser. This interaction was then analysed by construction of a z-stack with $600-700 \mathrm{~nm}$ optical slices (15-20 pcs) in a time lapse experiment for $20 \mathrm{~min}$. It turned out to be crucial not to add too much ODVs to be able to clearly discriminate individual ODVs over time. The images were analysed by using Zen (blue) 2.3 lite software.

\section{Acknowledgements}

We like to thank Dr Norbert de Ruijter (Wageningen University and Research) for his technical assistance with the confocal microscope and Els Roode for the rearing of the S. exigua larvae. We also acknowledge Felix Evers for construction of the bacmid for the generation of fluorescent mut-pif9 ODVs, and Wendy van Elst and Priyanka Thaoroijam for their assistance with midgut cell isolation and assay optimization. This work was supported by grant 824.14.16 of the Netherlands Organization for Scientific Research (NWO). 


\title{
Chapter 6
}

\section{The C-termini of baculovirus per os infectivity factors 1 and 2 mediate ODV oral infectivity by facilitating the binding of PIF0 and PIF8 to the core of the entry complex.}

Bob Boogaard, Jan W. M. van Lent, Monique M. van Oers

\begin{abstract}
Oral infection of caterpillars by baculoviruses is initiated by occlusion-derived virus particles (ODVs) that infect midgut epithelium cells. The ODV envelope therefore contains at least ten different proteins, which are called per os infectivity factors (PIFs). Nine of these PIFs form the so-called ODV entry complex that consists of a stable core formed by PIF1, 2, 3 and 4, to which the other PIFs (PIF0, 6, 7, 8 and 9) bind with lower affinity. PIF1 and 2 are not only essential for complex formation, but also mediate ODV-binding to the epithelial cell brush border, probably via the C-termini. To study the involvement of these PIFs during midgut infection in greater detail, we assessed the oral infectivity and the ability to form the complex of a series of PIF1 and PIF2 C-terminal truncation mutants of Autographa californica multiple nucleopolyhedrovirus (AcMNPV). Limited truncation of either PIF1 or 2 already severely impaired the ODV oral infectivity, but did not affect formation of the core complex. However, the entry complex as a whole was not assembled by these mutants as PIF0 and 8 failed to bind to the core. This suggests that the interactions between PIF1 and 2 in the core and PIF0 and PIF8 are important for the ODV infectivity and that complex formation complicates the determination of the exact roles of PIF1 and 2 during midgut infection. We also showed that the presence of PIF0, 6 and the ZF-domain of PIF8 are crucial for formation of the entry complex.
\end{abstract}




\section{Introduction}

Baculoviruses are double stranded DNA viruses that orally infect the larvae of lepidopteran, hymenopteran and dipteran insect species when they eat plant material that is contaminated with viral occlusion bodies (OBs) (Williams et al., 2017). The protein matrix of the OBs dissolves in the highly alkaline environment of the midgut lumen, resulting in the release of occlusion derived viruses (ODVs). The ODVs then bind and fuse with the microvilli of midgut epithelial cells to initiate a primary infection of the larval midgut. The infected epithelial cells produce a second type of virus particles, the budded viruses (BVs), which systemically spread the infection to other tissues in the larva.

The entry mechanism of ODVs into midgut epithelial cells is poorly understood due to its complexity as it involves at least ten different viral proteins. As each of these proteins is of specific importance for the virus oral infectivity, they are called per os infectivity factors (PIFs). In the Autographa californica multiple nucleopolyhedrovirus (AcMNPV), the ten PIFs and their specific genes are: PIF0 (or P74, encoded by the AcMNPV gene ac138) (Faulkner et al., 1997), PIF1 (ac119) (Kikhno et al., 2002), PIF2 (ac22) (Pijlman et al., 2003), PIF3 (ac115) (Ohkawa et al., 2005), PIF4 (ac96) (Fang et al., 2009), PIF5 (ODV-E56/ac148) (Sparks et al., 2011a), PIF6 (ac68) (Nie et al., 2012), PIF7 (ac110) (Liu et al., 2016), PIF8 (P95/ac83) (Zhu et al., 2013) and PIF9 (ac108) (Wang et al., 2019, Chapter 4). When OBs of a pif deletion mutant are orally administered to larvae, the infection fails. However, in cultured insect cells the deletion of a pif gene does not affect the production of ODVs or OBs, nor the production and infectivity of BVs. PIF8 is the single exception as this protein is besides crucial for oral infectivity also involved in nucleocapsid assembly (Zhu et al., 2013, Javed et al., 2017). These two functions are performed by two separate domains, of which the zinc-finger (ZF) domain is crucial for oral infectivity (Javed et al., 2017).

Nine of the ten PIF proteins form a large complex in the ODV envelope. This so-called ODV entry complex consists of a stable core, formed by PIF1-4 (Peng et al., 2010, 2012), and five other PIFs (PIF0 and PIF6-9) that bind to the complex with lower affinity (Peng et al., 2012, Wang et al., 2019, Chapter 2, 3, 4). PIF5 is not a component of the entry complex (Peng et al., 2012). PIF1-3 are essential for formation of the stable core and still interact with each other in absence of PIF4 (Peng et al., 2010, 2012). Furthermore, the core complex appears to be highly stable, since it remained intact when isolated ODVs were partially denatured by treatment with $8 \%$ SDS and 5\% 2-mercaptoethanol and heating up to $75^{\circ} \mathrm{C}$ (Peng et al., 2010, Chapter 3). 
The complete entry complex however, can only be found under non-denaturing conditions as the more loosely associated components dissociate from the core upon treatment with SDS. Therefore, PIF0, 6, 7, 8 and 9 were only detected as part of the entry complex by blue nativePAGE analysis and by mass spectrometry as co-immune precipitants of PIF1 (Peng et al., 2012, Wang et al., 2019, Chapter 2, 3, 4). Further examination of the entry complex under nondenaturing conditions also showed that PIF4, the ZF-domain of PIF8 and PIF9 are essential for formation of the complex (Peng et al., 2012, Javed et al., 2017, Wang et al., 2019, Chapter 4).

PIF0, 1 and 2 have been shown to be required for the binding of ODVs to the brush border of the midgut epithelial cells (Haas-Stapleton et al., 2004, Ohkawa et al., 2005, Mu et al., 2014) and were indeed indicated to point outwards from the ODV envelope in immunogold labelling analyses (Peng et al., 2010). This corresponds with in silico data, in which these proteins are predicted to point outwards from the ODV envelope, making these available for interaction with the larval host in the midgut (Chapter 2). PIF0 was predicted to be inserted in the ODV envelope via its $\mathrm{C}$-terminal transmembrane domains, so that the $\mathrm{N}$-terminal parts of this protein point outwards. PIF1 and 2 are predicted to be N-terminally inserted in the ODV envelope, with their C-termini sticking out.

In addition to being important for ODV binding, PIF1 and 2 are essential for formation of the entry complex. This raised the question on whether these PIFs are directly involved in ODVbinding via the exposed C-termini, or indirectly by the binding of other PIF proteins to these C-termini. Most baculoviruses have a narrow host range and the ODV binding capacity is one of the host range determinants, as the resistance of Spodoptera frugiperda larvae to oral infection with AcMNPV was attributed to aberrant ODV binding when compared to the Spodoptera frugiperda multiple nucleopolyhedrovirus (Haas-Stapleton et al., 2005). The amino acid sequences of PIF1 and PIF2 of these two alphaviruses are highly similar, but show (slightly) more variation at the C-terminal ends than at the $\mathrm{N}$-terminal parts (See alignments and truncation sites in Supplemental data Fig. S1 and S2). It was therefore hypothesized that the more variable C-terminal parts of PIF1 and 2 are involved in ODV binding and the more conserved N-terminal parts in complex formation. This hypothesis was tested by the construction a series of AcMNPV PIF1 and PIF2 C-terminal truncation mutant viruses, of which the ODVs were analysed for the presence of the core and entry complex and for oral infectivity. This revealed that upon truncation of one of these PIFs, the two loosely associated components PIF0 and 8 failed to bind to the core and oral infectivity was abolished. The involvement of PIF0, 4, 6 and PIF8 in complex formation were also studied. 


\section{Results}

\section{Construction of PIF1 and PIF2 C-terminal truncation mutants of AcMNPV}

To determine the involvement of the C-terminal parts of PIF1 and PIF2 in oral infectivity and complex formation, a series of recombinant AcMNPV viruses with C-terminally truncated versions of either PIF1 or PIF2 were generated. These viruses were constructed by repairing pif1 and pif2 deletion bacmids via Tn7-mediated transposition using modified pFastBacDual (pFBD $\Delta$ p10-polh) vectors that contain full or shorter versions of the pif1 or pif2 open reading frames (ORFs) under control of their endogenous promoters, as previously described in Peng et al., 2010. The AcMNPV PIF1 protein that consists of 530 amino acids was step-wise truncated from the C-terminus until amino acid V459, F423 and A314, respectively (Fig. 1). For PIF2, that has 382 amino acids in the wild type virus, the C-terminal truncation mutants N358 and P351 were constructed (Fig. 2). The truncation sites were chosen based on the presence of conserved amino acid motifs in the C-terminus. The pFBD-vectors also contained the polyhedrin (polh) ORF downstream of its own promoter to enable the mutant viruses to form OBs (See alignments and truncation sites in Supplemental data Fig. S1 and S2).

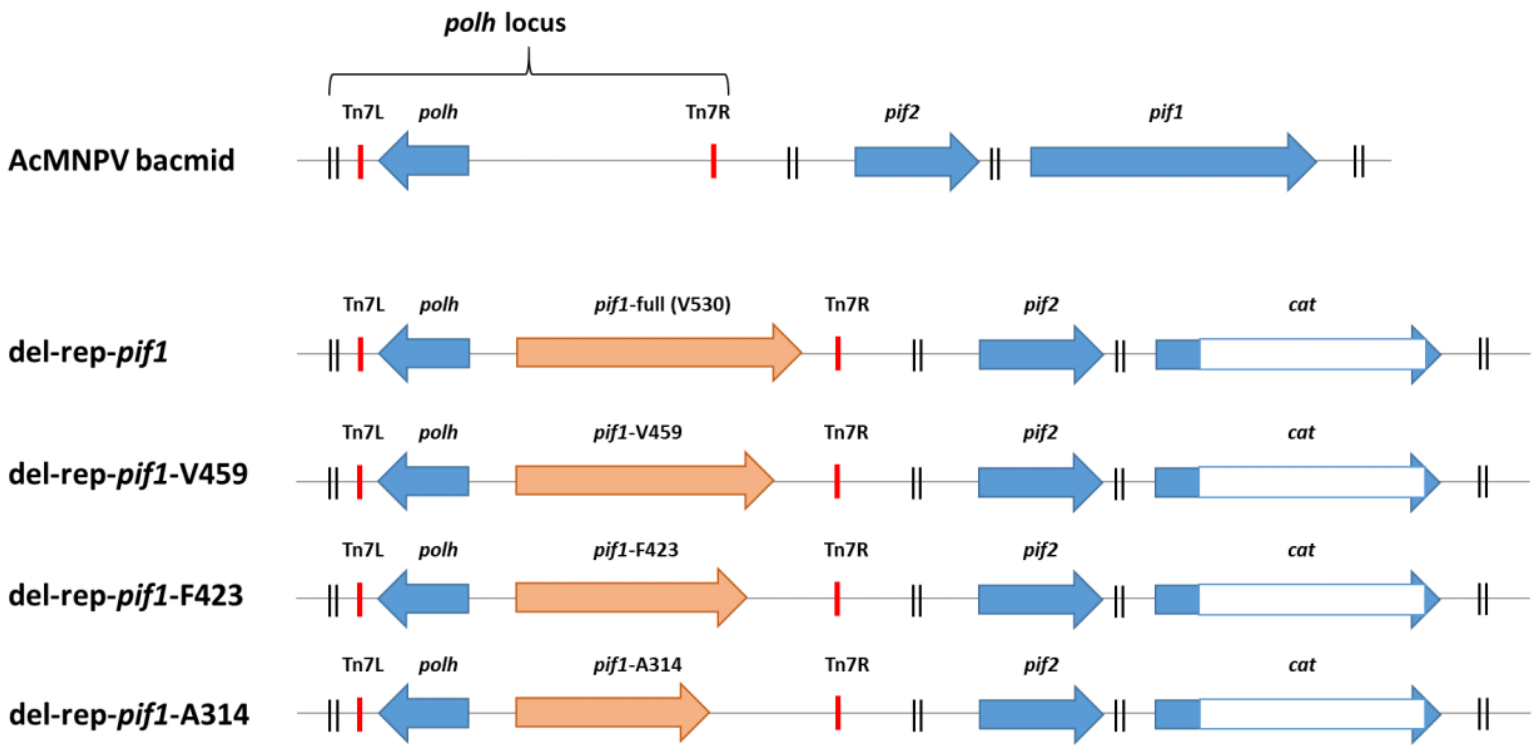

Figure 1: Overview of constructed bacmids of the pif1 truncation mutants. The pifl deletion bacmids (described in Peng et al., 2010) were repaired with shorter versions of the respective open reading frames (ORF) via Tn7 mediated transposition into the polyhedrin (polh) locus. The bacmids also contain the polh ORF under its own promoter to enable the resulting viruses to produce OBs. The directions of the ORFs in the genome are indicated by the arrows. The bacmids of the pifl truncation mutants and the del-rep-pifl repair mutant, containing the full-length ORF. In the del-rep-pifl-V459, the deletion bacmid was repaired with the pif1 ORF that was truncated at the 3 'end until the codon that corresponds with Valine 459. The bacmids del-rep-pif1-F423 and -A314 contain the shortened ORF that corresponds with Fenylalanine 423 and Alanine 314, respectively. 


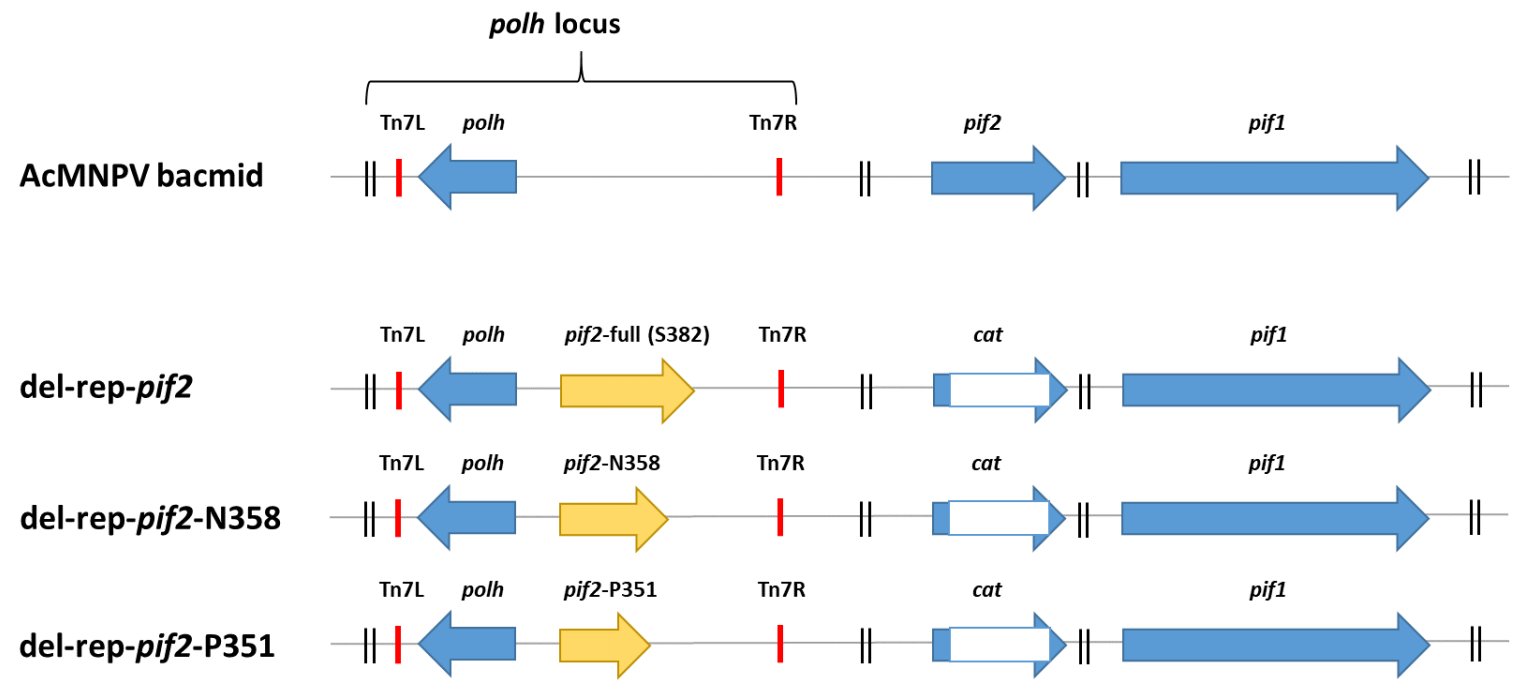

Figure 2: The bacmids of the pif2 truncation mutants. Del-rep-pif2 bacmid is a repair mutant, containing the full-length ORF, while the del-rep-pif2-N358 and -P351 bacmids contain 3'end truncated versions of the pif2 ORF, corresponding with Asparagine 358 and Proline 351 respectively.

C-terminal truncations of PIF1 and PIF2 impair oral infectivity in Spodoptera exigua larvae.

Oral infectivity of the PIF1 and PIF2 truncation mutants was tested by oral inoculation of early L3 S. exigua larvae with a sucrose solution containing $3 \times 10^{8} \mathrm{OBs} / \mathrm{ml}$. This showed that the full-length repair viruses were able to infect the larvae, resulting in their liquefaction, while the oral infectivity of all the truncation mutants was severely affected (Table 1). Truncation mutants pif1-V459 and pif1-A314 showed no mortality whereas with pif1-F423, a single larva out of 24 died in experiment 3 (Table 1). Inoculation with pif2-N358 showed low a level of mortality in all three bioassays and pif2-P351 resulted in a single larval death. To confirm that the liquefied larvae indeed had been infected by the truncation mutant under study, the larvae were analysed by PCR with various primer pairs, in which the reverse primer annealed either at or downstream of one of the truncation sites (See Supplementary data Fig. S3 and S4). This showed that the larvae inoculated with the pif1-F423 and pif2-P351 mutant viruses were

Table 1: Mortality of S. exigua larvae after oral inoculation with OBs

\begin{tabular}{cccc}
\hline Virus & Exp. 1 & Exp. 2 & Exp. 3 \\
\hline 3.0 x $\mathbf{~ 1 0}^{\mathbf{8}}$ OBs / ml $)$ & & & $0 / 24$ \\
mock & $0 / 24$ & $0 / 24$ & $21 / 21$ \\
rep-pif1 & $20 / 22$ & $23 / 24$ & $0 / 24$ \\
-V549 & $0 / 24$ & $0 / 24$ & $1 / 24$ \\
-F423 & $0 / 23$ & $0 / 24$ & $0 / 24$ \\
-A314 & $0 / 24$ & $0 / 24$ & $22 / 23$ \\
rep-pif & $21 / 24$ & $22 / 24$ & $3 / 24$ \\
-N358 & $1 / 23$ & $3 / 24$ & $1 / 24$ \\
-P351 & $0 / 24$ & $0 / 24$ & \\
\hline
\end{tabular}


indeed infected by the corresponding mutant virus. However, the larvae that died from inoculation with pif2-P358 showed to be infected by the repair or wild type virus. The virus stock that was used to inoculate the larvae was also checked and did not contain wild type or repair virus. These findings confirm that the larvae were indeed incidentally infected by the corresponding truncation mutants, but the C-terminal parts of PIF1 and 2 are nevertheless important for ODV oral infectivity.

PIF1 and PIF2 C-terminal truncation mutants form the stable core complex.

To test whether the truncated PIF1 and PIF2 proteins are routed to the ODV envelope and still able to form the stable core complex, the envelope fractions of the truncation mutants were analysed by SDS-PAGE and western blotting with antisera against either PIF1 or PIF2. It was previously shown that the core could be detected as a $170 \mathrm{kDa}$ band with these antisera when a sample of ODV envelope proteins was incubated in Laemmli buffer at $50^{\circ} \mathrm{C}$, instead of $95^{\circ} \mathrm{C}$ (Peng et al., 2010). When the ODV envelope fractions of the PIF1 truncation mutants were analysed with PIF1 antiserum under these conditions, the core was found for the truncation mutants pif1-A459 and pif1-F423, but not for the pif1-A314 mutant (Fig. 3). In the latter, the PIF1 antiserum only found the truncated PIF1 monomers between 35 and $40 \mathrm{kDa}$ heights. The $40 \mathrm{kDa}$ band was more prominent after partial denaturation at $50^{\circ} \mathrm{C}$ and the $35 \mathrm{kDa}$ band after complete denaturation at $95^{\circ} \mathrm{C}$. This indicates that this longer C-terminal truncation affected the protein conformation of the half-life of PIF1. The core complex was also found in

\section{anti-PIF1}

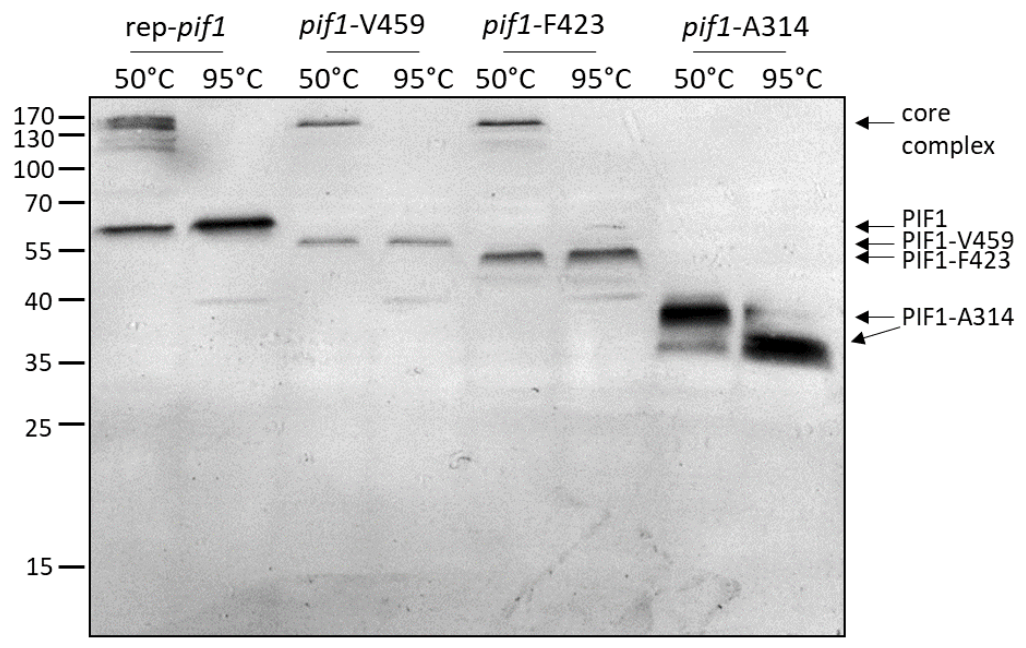

Figure 3: Western blot analysis of the ODV envelope fractions of the pif1 repair and truncation mutants with PIF1 antiserum. The ODV envelope proteins were extracted by incubation in extraction buffer, containing $0,5 \%$ Triton-X100, incubated in Laemmli buffer at either 50 or $95^{\circ} \mathrm{C}$ for $10 \mathrm{~min}$. and separated in SDS-PAGE gels. The core complex and PIF monomers are indicated by arrows. 


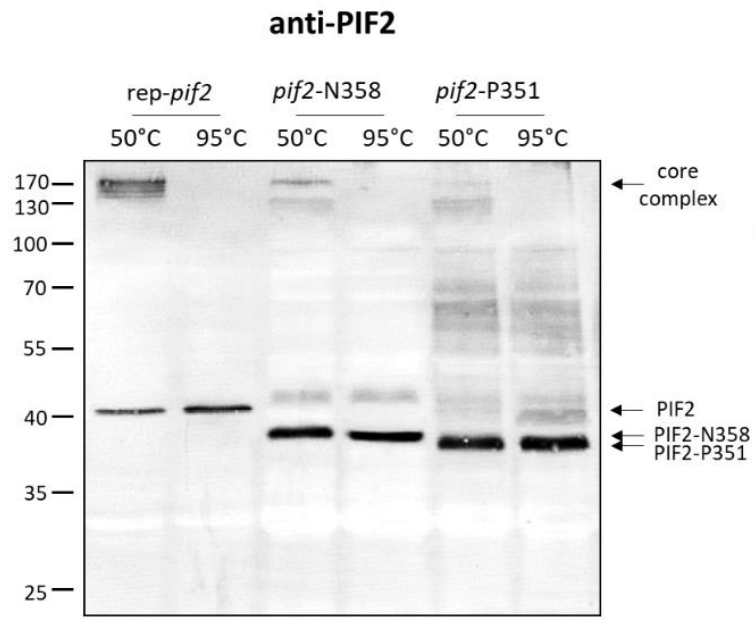

(a)

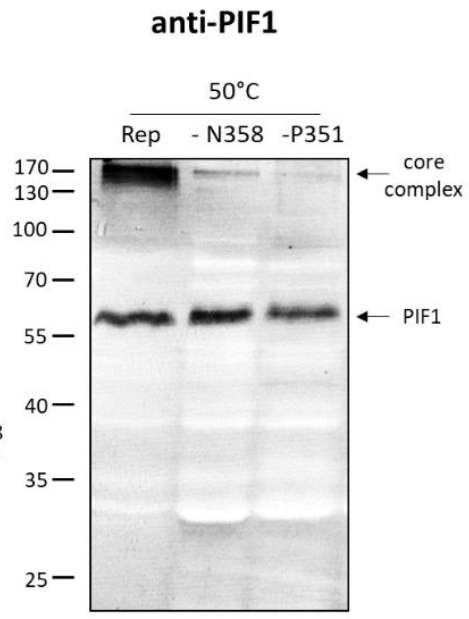

(b)

Figure 4: Western blot analysis of the ODV envelope fractions of the pif2 truncation and repair mutants. For these analyses, antiserum against PIF2 was used (a). The presence of the core complex was validated with PIF1 antiserum (b).

both pif 2 truncation mutants under these conditions by using either PIF1- or PIF2 antiserum, although in lower quantities than in the repair mutant for unknown reasons (Fig. 4).

When the envelope proteins were incubated at $95^{\circ} \mathrm{C}$, the core complex dissociated completely and only PIF1 or PIF2 monomers were found. In the full-length repair mutants, the monomers were found at the expected heights in the gel, PIF1 at $60 \mathrm{kDa}$ and PIF2 at $40 \mathrm{kDa}$ height. After truncation, monomers were found with lower mass as expected, corresponding with the extend of the truncation, i.e. the larger the truncation, the smaller the monomers. These analyses showed that the C-terminal truncated forms of PIF1 and PIF2 were properly targeted to the ODV envelope and that all mutants except pifl-A314 were able to form the core complex, although in smaller quantities after truncation of PIF2.

PIF1- and PIF2 truncation mutants lack the ODV entry complex.

The ODV envelope proteins were also analysed under non-denaturing conditions by blue-native PAGE to determine whether the PIF1 and PIF2 truncation mutants were still able to form the ODV entry complex. Previous studies showed that the entry complex was found as a double band running at roughly the same height as the $480 \mathrm{kDa}$ marker, when using antiserum against one of its components (Peng et al., 2012). When using antisera against one of the core components (PIF1 and PIF2), or the loosely associated component (PIF8), the ODV entry complex was detected in the pifl- and pif2 full length repair viruses, but not in any of the truncation mutants (Fig. 5). Analysis with PIF8 antiserum was also used as loading control by the detection of PIF8 dimers between the 242 and $146 \mathrm{kDa}$ markers, as reported before (Wang 


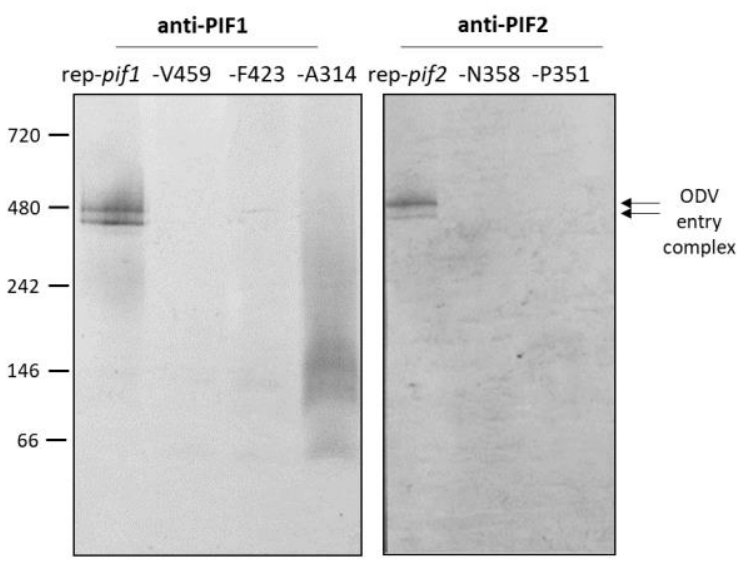

(a)

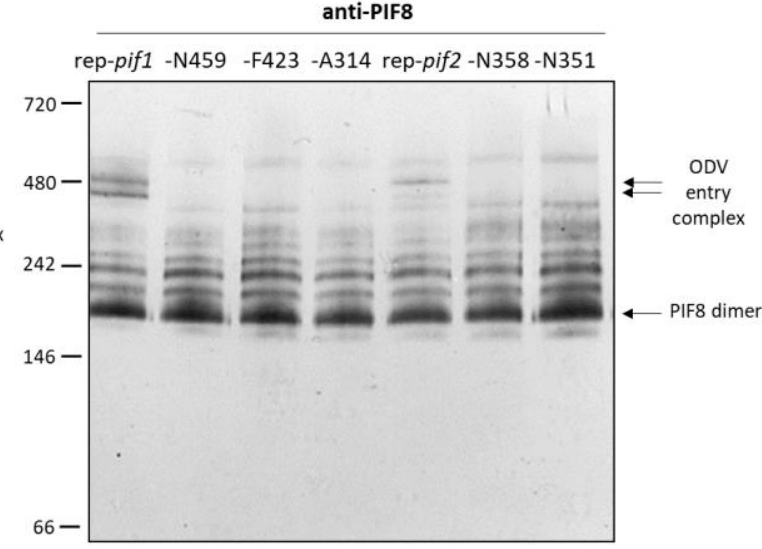

(b)

Figure 5: Blue native-PAGE and western blot analysis of the ODV envelope fraction of the pif1 and pif2 repair and truncation mutants. The ODV envelope proteins were separated under non-denaturing conditions in a BN-PAGE gel and analysed by western blotting with antiserum against PIF1 or 2 (in the core) (a) and antiserum against the loosely associated protein PIF8 (b). The entry complex and the PIF8 dimers (used as loading control) are indicated by arrows.

et al., 2019) (Fig. 5b). The core was not detected by this method in any of the truncation mutants. These results indicate that the C-termini of PIF1 and PIF2 are essential for formation of the entry complex.

\section{C-terminal truncation of PIF1 or PIF2 affects the interaction with PIFO and PIF8.}

To determine why the formation of the ODV entry complex was abolished after C-terminal truncation of either PIF1 or PIF2, while the core was still present, a co-immunoprecipitation study was performed with the PIF1 antiserum on ODV envelope fractions of the pifl-F423 and pif2-P351 truncation mutants. The pif1 repair virus was used as positive control and the pifl deletion virus as a negative control for the precipitation of PIF1. The immuno-precipitations were first analysed by western blot with antisera against PIF1, to verify that this protein indeed precipitated; then against PIF2 to confirm the interaction of PIF1 with PIF2 in the core complex; and finally with antisera against PIF0 and PIF8 to analyse the interaction with these two largest (loosely associated) components of the entry complex (Fig. 6). PIF1 was found to be precipitated from ODV envelope fractions of the pif1 repair mutant and the pif2-P351 truncation mutant, but not from the pifl deletion mutant. After immunoprecipitation, PIF1 was found to run higher than in the input sample, probably due to the presence of large amounts of immunoglobulin that also eluted from the beads. Pre immune serum failed to precipitate PIF1, indicating that the PIF1 antiserum specifically precipitated its target protein (Fig. 6a). Unfortunately, precipitation of the truncated PIF1-F423 could not be confirmed directly as this 

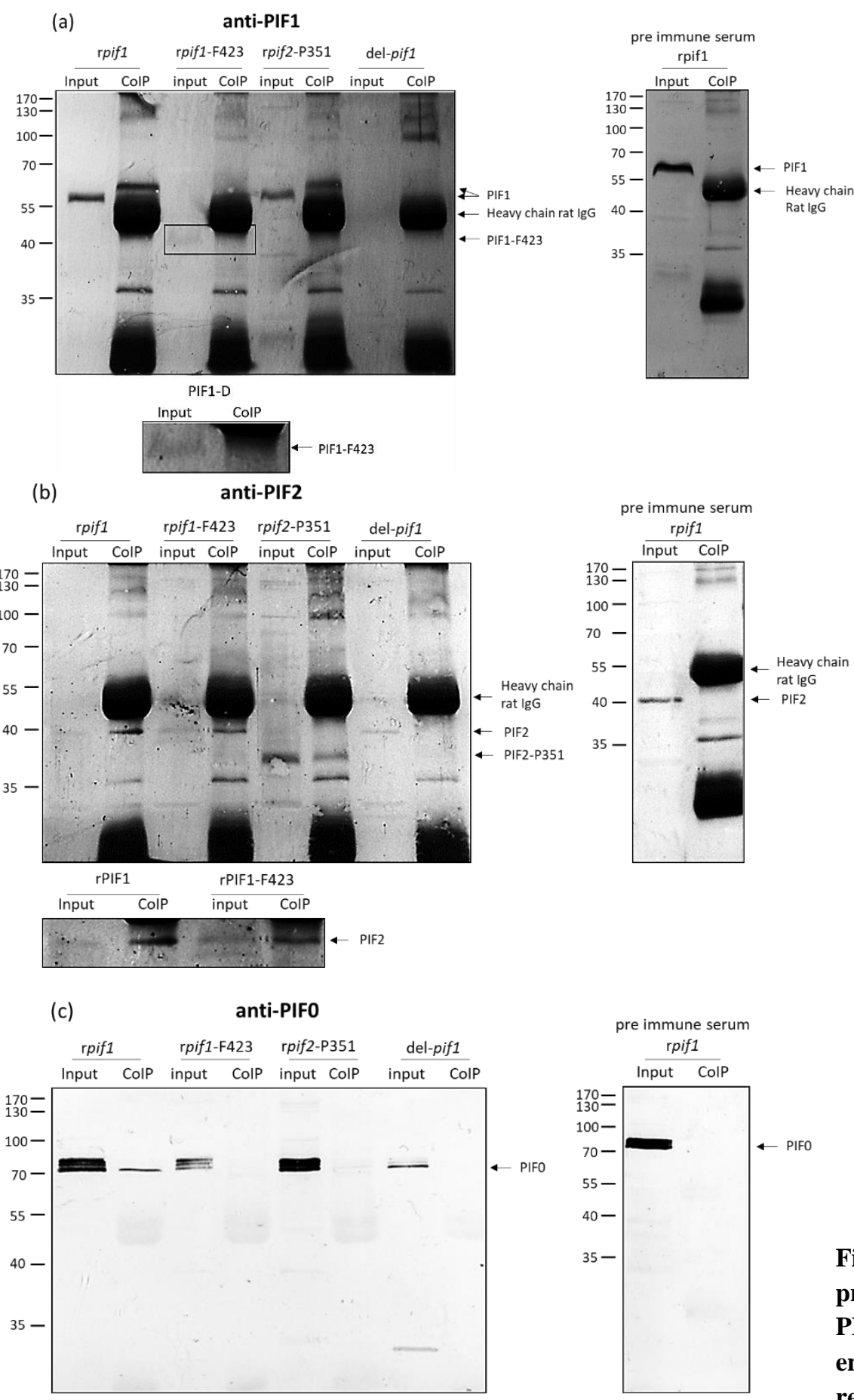

(d)

\section{anti-PIF8}

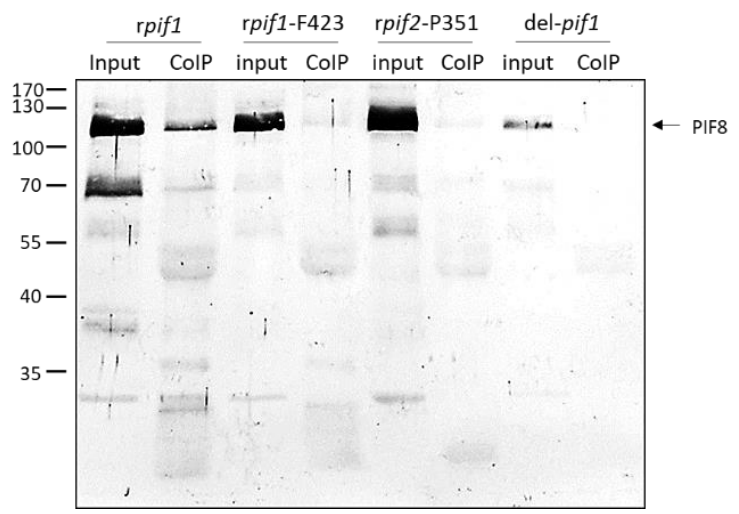

pre immune serum

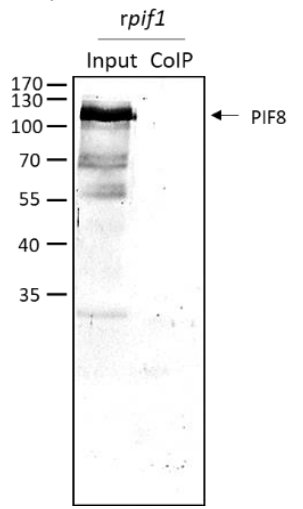

Figure 6: Coimmuno-

precipitation analysis with PIF1 antiserum on the ODV envelope proteins of the pif1 repair, pif1-F423 and pif2P351 truncation mutants. An immunoprecipitation on a pif1 deletion mutant and a precipitation with pre-immune serum on the pifl repair virus were used as negative controls. The input and eluted proteins were separated in SDS-PAGE gels and analysed on western blots with antiserum against PIF1 (a), PIF2 (b), PIF0 (c) and PIF8 (d). 
protein had approximately the same migration rate as the rat immunoglobulin heavy chain (Fig. 6a), which was also recognized by the secondary antibodies during western blot analysis. PIF2 co-precipitated with PIF1 in the pif1 repair mutant, also after its truncation (see the pif2P351 truncation mutant), and with the pifl-F423 truncation mutant, but not in the pif1 deletion mutant or after usage of pre immune serum. This indicates, although indirectly, that the shorter version of PIF1 (F423) was also precipitated by the PIF1 antiserum and confirmed that the interaction between PIF1 and PIF2 remains intact after C-terminal truncation of one of these PIFs (Fig. 6b). On the contrary, the loosely associated PIF0 and PIF8 only co-precipitated in the pif1 repair virus, so with full length PIF1, but not with any of the truncated versions (Fig. 6c, d). PIF0 was detected as multiple bands between 70 and approximately $85 \mathrm{kDa}$ in the input samples, suggesting that this protein is post-translationally modified and occurs in multiple forms in the ODV envelope. These forms are separated in these analyses, because of the relatively long running times that we used to separate the immunoglobulins from the target proteins. However, after immunoprecipitation of PIF1, only a $70 \mathrm{kDa}$ band was found. Whether the other forms of PIF0 are below detection level or whether the complex contain only one form of this protein was not further analysed. The results indicate that the C-termini of PIF1 and PIF2 are essential for formation of the ODV entry complex by facilitating the interaction between these PIFs present in the stable core and at least two of the loosely associated PIFs, namely PIF0 and PIF8.

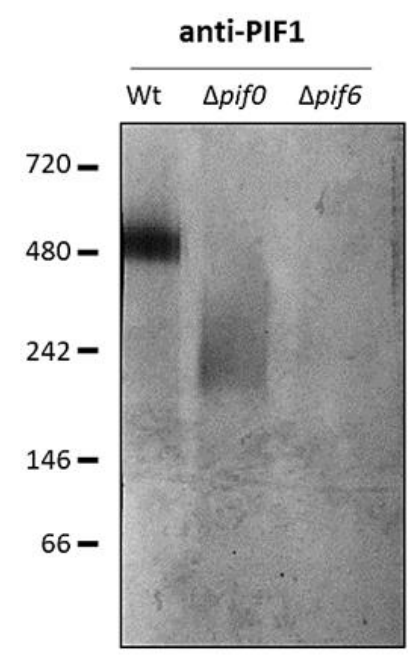

(a)

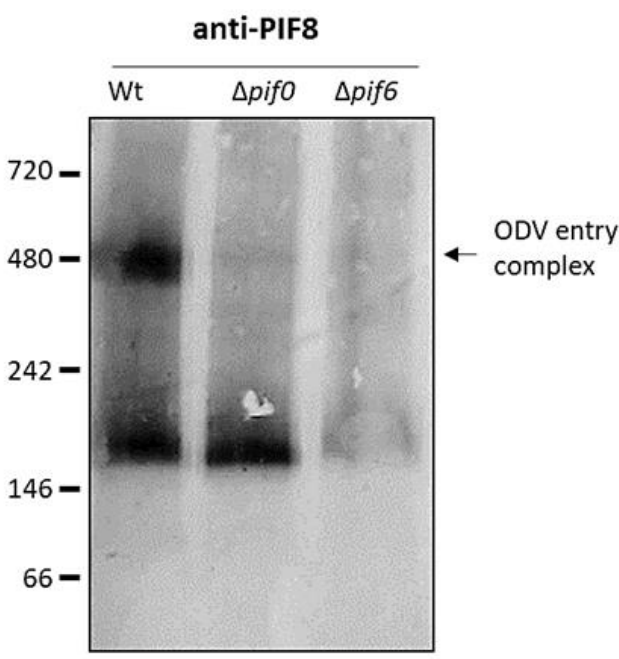

(b)

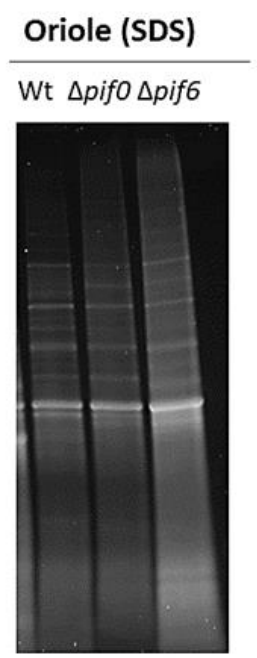

(c)

Figure 7: Blue native-PAGE analysis of the ODV envelope fractions of the pifo and pif6 deletion mutants. The ODV envelope proteins were separated in a BN-PAGE gel and analysed by western blotting with antiserum against PIF1 (a) and PIF8 (b). The entry complex is indicated by the arrows. Part of the ODV envelope fractions were incubated at $95^{\circ} \mathrm{C}$, separated in SDS-PAGE gels and stained with Oriole as loading control (c). 
PIF0, PIF4 and PIF6 facilitate the interaction between PIF1 in the stable core and PIF8.

To determine whether the loosely associated PIFs only need an intact core complex for binding or whether this also requires the presence of other loosely associated PIFs, the ODV-envelope fractions of pifO- and pif6 deletion mutants were analysed by blue-native PAGE and by coimmunoprecipitation with PIF1 antiserum. The entry complex was detected in wild type AcMNPV with antisera against PIF1 and PIF8, but not in absence of either PIF0 or 6 (Fig. 7). In the pifO deletion mutant, PIF1 containing complex(es) was found around $242 \mathrm{kDa}$ height with very low resolution. This might be the core complex as described in Wang et al., 2019. These data show that PIF0 and PIF6 are crucial for formation of the entry complex.

For the co-immunoprecipitation study with PIF1 antiserum, a pif4 deletion mutant was also included as an earlier study had shown that a smaller complex is formed by PIF1 to 3 in absence of PIF4, but nevertheless failed to form the entry complex (Peng et al., 2012). Pre-immune serum was used as negative control for the precipitation of PIF1 and the co-precipitation of PIF2, PIF0 and PIF8 (Fig. 8). Western blot analysis with PIF1 antiserum showed that the target protein indeed precipitated in wild type and pif4, pifO and pif6 deletion mutants and was not found precipitated by the pre-immune serum (Fig. 8a). In the pif4- and pif6 deletion mutants, PIF2 and PIF0 were found precipitated with PIF1, but not PIF8. (Fig. 8b-d). In the pif0 deletion mutant, only PIF2 precipitated with PIF1 (Fig. 8b). These analyses indicate that the binding of PIF0 to the core is not dependent on the presence of PIF4 or the binding of PIF6, and possibly PIF8 (as this PIF failed to bind the complex in all these mutant viruses). However, the binding of PIF8 to the stable core requires the presence of PIF4 in the core and at least the binding of PIF0 and 6.

\section{The zinc finger domain of PIF8 interacts with PIFO and PIF1.}

A working model has been proposed for the function of PIF8, in which the zinc finger (ZF-) domain recruits and promotes assembly of PIF proteins in the ODV envelope to form the entry complex (Javed et al., 2017). To validate the presence of the PIFs in the ODV envelope of a PIF8 $\triangle$ ZF-HA mutant (lacking this domain), the ODV-envelope fractions of this mutant and a pif8-HA repair virus were analysed by western blotting with anti-HA antibodies and antisera against either PIF0 or PIF1. When using anti-HA antibodies, PIF8 and the $\triangle$ ZF mutant were detected as >100 and $70 \mathrm{kDa}$ bands respectively, implying their presence in the ODV-envelope (Fig. 9a). Some smaller bands were also detected (below the 40 and $35 \mathrm{kDa}$ marker), which were previously detected by the PIF8 antiserum as well (Fig. 4). That these bands are now also 
(a)

anti-PIF1
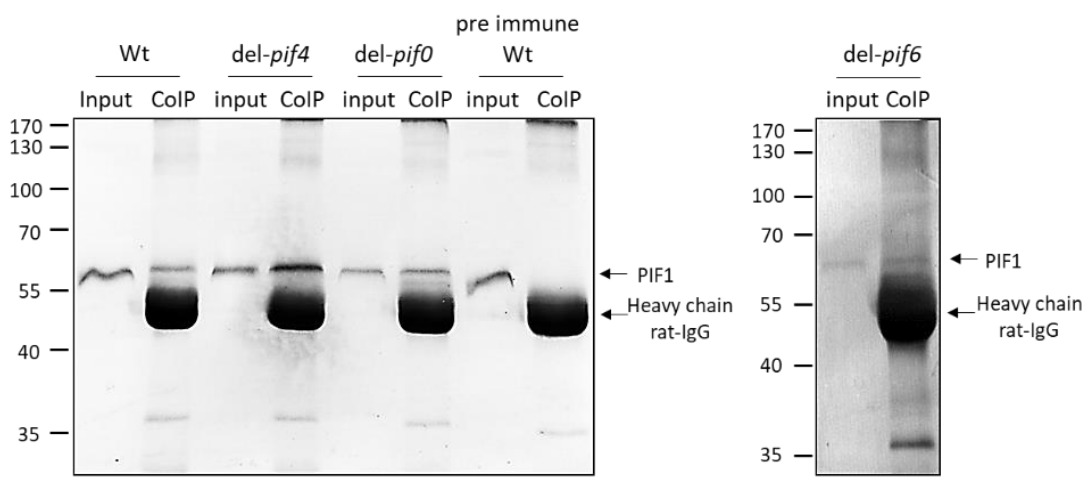

(b)

anti-PIF2
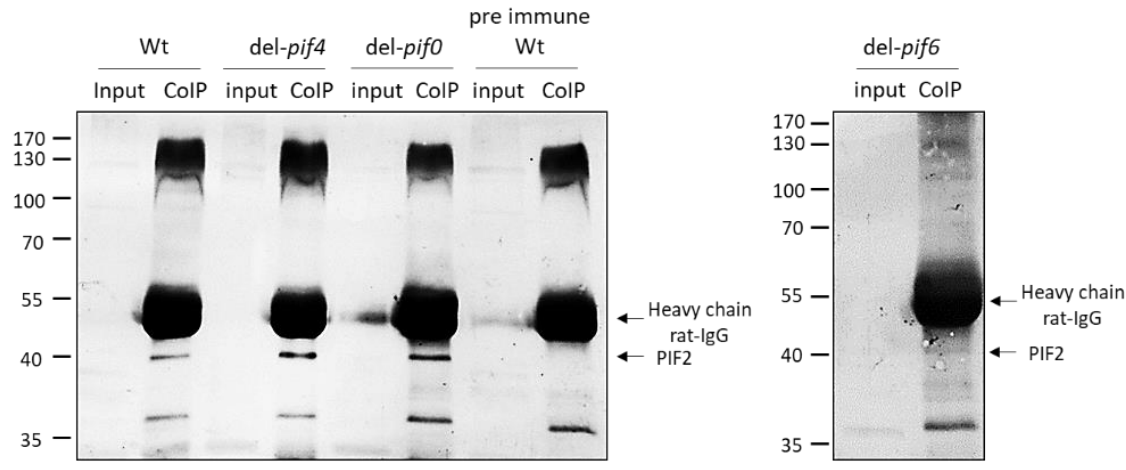

(c)

anti-PIFO
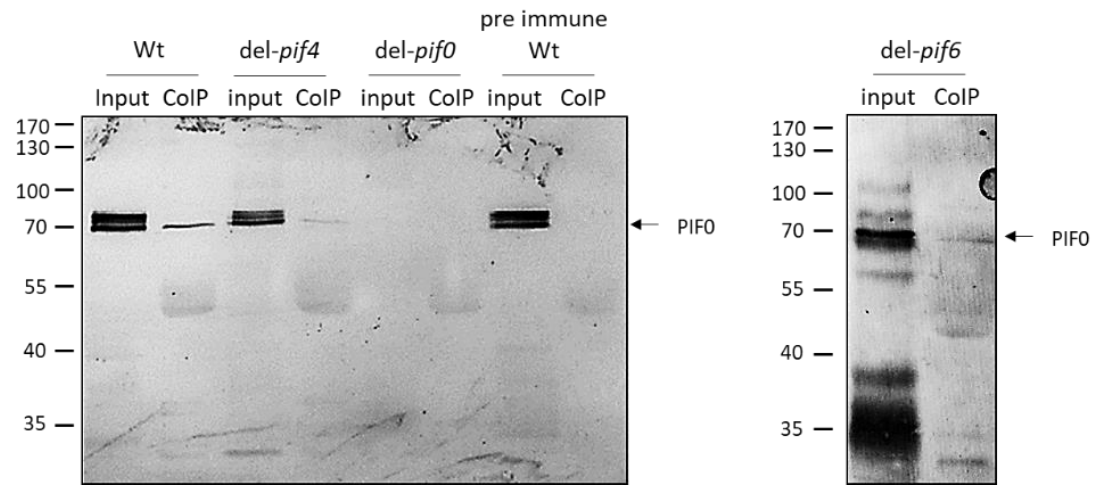

(d)

anti-PIF8
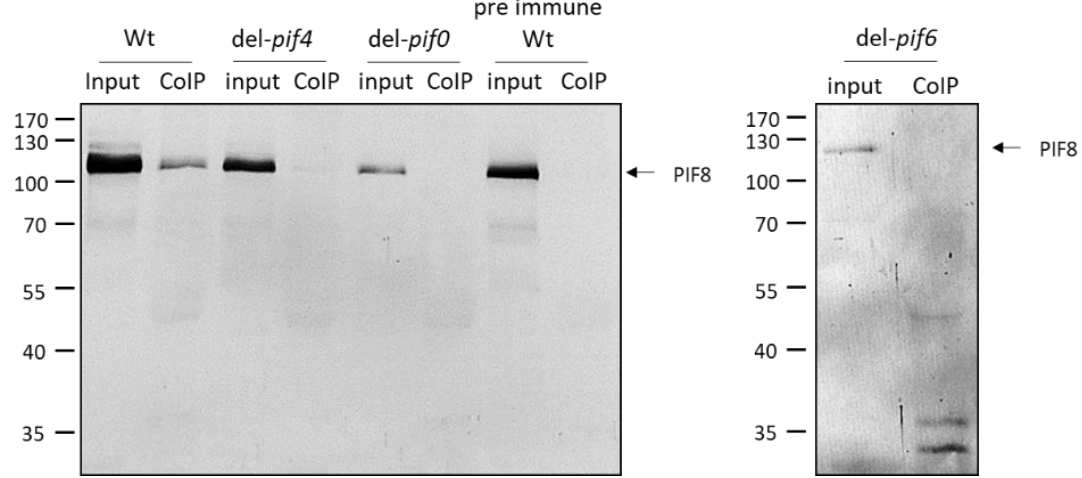

Figure 8: A co-immunoprecipitation analysis with PIF1 antiserum on the ODV envelope proteins of pif0, pif4 and pif6 deletion mutants. Precipitation with pre immune serum was used as negative control. The input and eluted proteins were separated in SDS-PAGE gels and analysed by western blot with antiserum against PIF1 (a), PIF2 (b), PIF0 (c) and PIF8 (d). PIF2 was only detected in the concentrated protein sample after elution from the anti-HA beads and not in the diluted input sample (b). 


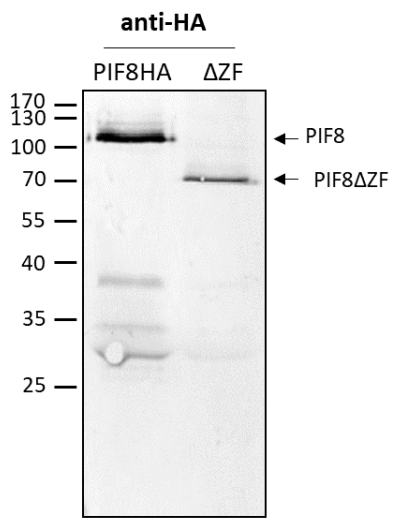

(a)

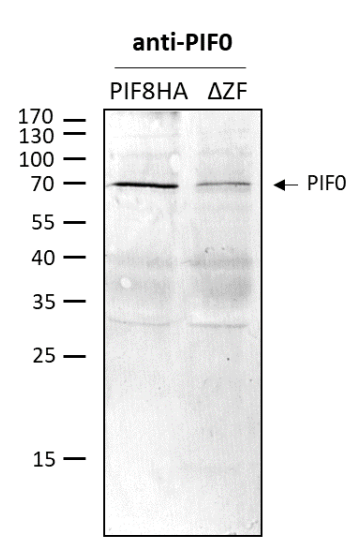

(b)

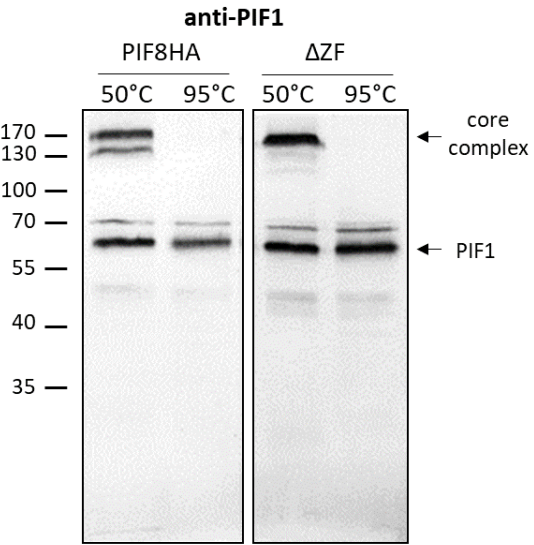

(c)

Figure 9: Western blot analysis of the ODV envelope fractions of an HA-tagged PIF8 repair mutant and an HAtagged PIF8 mutant that lacks the zinc-finger (ZF-) domain with antibodies against the HA-tag (a), PIF0 antiserum (b) and PIF1 antiserum (c). Part of the ODV envelope fractions were incubated at $50^{\circ} \mathrm{C}$ for detection of the 170 $\mathrm{kDa}$ core complex.

found with anti-HA antibodies suggests that these bands are specific for PIF8. These lower bands were also found by others (personal communication Theilmann). With antisera against either PIF0 or PIF1, 70 and $60 \mathrm{kDa}$ bands were detected respectively, confirming the presence of these PIFs in the ODV-envelope of both the repair and ZF-mutant virus (Fig. 9b, c). Furthermore, when the protein sample was incubated at $50^{\circ} \mathrm{C}\left(\right.$ instead of $95^{\circ} \mathrm{C}$ ) and analysed with PIF1 antiserum, the core complex was found, indicating that in addition to PIF1, also PIF2, 3 and 4 were present in the ODV envelope in these mutants.

As the ZF-domain of PIF8 was shown to be essential for formation of the entry complex (Javed et al., 2017), the importance of this domain for the interaction with other PIFs was studied by a co-immunoprecipitation study with anti-HA magnetic beads. The eluted proteins were analysed by western blotting with anti-HA antibodies and confirmed the precipitation of the HA-tagged PIF8 itself (Fig. 10a). The blots were also analysed with antisera against PIF0 or PIF1, to validate the interaction of PIF8 (either direct or indirect) with another loosely associated PIF (PIF0) and the stable core respectively. This showed that PIF0 and 1 coprecipitated with PIF8, but not after removal of the ZF-domain. This indicates that the ZF-domain of PIF8 is important for the interaction with other PIFs of the entry complex (Fig. 10b and c). These results also demonstrate that the ZF-domain of PIF8 is not involved in recruitment of the PIFs to the ODV envelope and confirm the importance of this domain for complex assembly. 


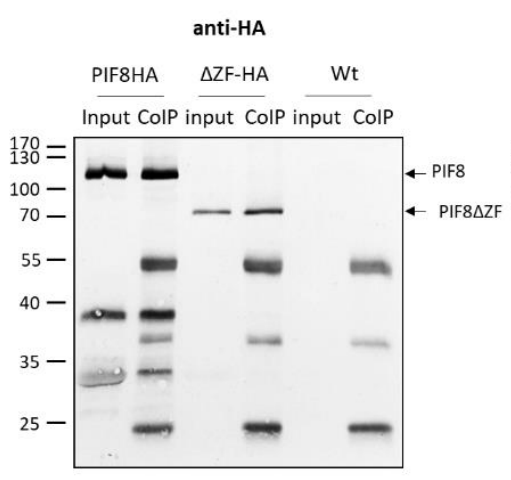

(a)

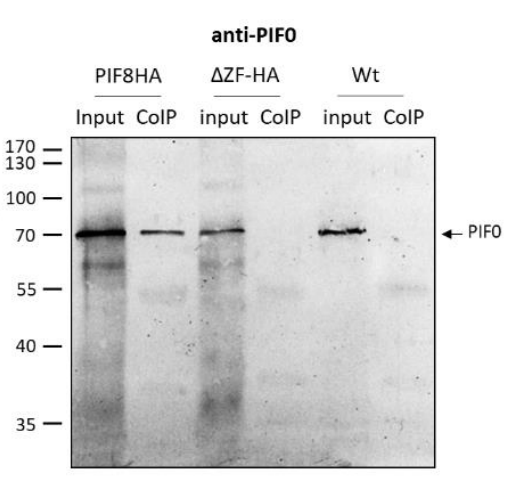

(b)

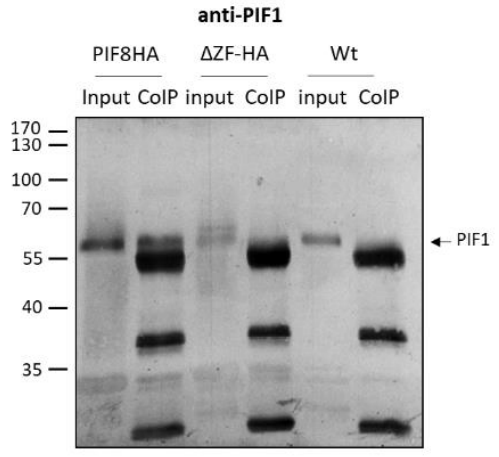

(c)

Figure 10: A co-immunoprecipitation study with anti-HA magnetic beads on the ODV envelope proteins of HA-tagged PIF8 and PIF8 $\triangle$ ZF mutant. The input and eluted proteins were analysed by SDS-PAGE and western blot with anti-HA antibodies (a), PIF0 antiserum (b) and antiserum against PIF1 (c). The ODV envelope of wild type AcMNPV (no HA-tag) was used as a negative control.

\section{Discussion}

Infection of larval epithelial midgut cells by baculovirus ODVs is initiated by at least ten different PIF-proteins, of which nine (all except PIF5) form an ODV entry complex, located in the ODV envelope. This complex consists of a stable core formed by PIF1 to 4 and five loosely associated PIFs, i.e. PIF0 (P74) and PIF6 to 9 (Peng et al., 2010, 2012, Wang et al., 2019, Chapter 2, 3, 4). That all these PIFs form a complex suggests that midgut infection requires a close collaboration between these proteins in a network of protein-protein interactions. This retrospectively complicates the interpretation of the results of earlier studies towards the functional role of individual PIFs using pif deletion mutants, as the function of non-targeted PIFs might be affected indirectly in these mutants. Previous studies with pifl and pif2 deletion mutants showed that these particular PIFs are important for the binding of ODVs to the midgut epithelium (Ohkawa et al., 2005). Later, these PIFs were also shown to be essential for the formation of the stable core of the entry complex (Peng et al., 2010). That PIF1 and 2 could be involved in interaction with the host is in accordance with in silico data that indicated that the C-termini of these PIFs point outwards from the ODV envelope (Chapter 2). However, whether these PIFs are directly involved in ODV binding or whether the binding was affected due to an inability to form the complex was not known.

To elaborate more on the involvement of PIF1 and 2 in midgut infection, we constructed a series of recombinant AcMNPVs, in which these PIFs were C-terminally truncated, and were analysed for oral infectivity and the capacity to form the core and entry complex. This showed that even limited truncation of PIF1 or 2 severely affected the oral infectivity of ODVs and that formation of the entry complex was abolished in all mutants, despite the fact that these truncated 
versions were still able to form the core-complex, except pifl-A314 (Table 1, Fig. 4-5). Immunoprecipitation studies showed that the truncations affected the binding of the loosely associated PIF0 and 8 (and possibly also the binding of other PIFs) to the core complex (Fig. 6). Since the pif1-A314 mutant was also defective in formation of the core complex, A314 is located in a domain that is important for the interaction with PIF2, 3 and 4. How far this domain extends $\mathrm{N}$-terminally is not known. The observations suggest that the truncated parts of the C-terminal ends of PIF1 and 2 are important for formation of the entry complex by facilitating the interaction between the stable core and the loosely associated PIF0 and 8. PIF4 appeared not to be crucial for the binding of PIF0 to the core, as in a pif4 deletion mutant, in which a smaller core complex is formed by PIF1 to 3 (Peng et al., 2012), PIF0 was still found to be associated (Fig. 8). That the C-termini of PIF1 and 2 are crucial for the binding of PIF0 and 8 to the core suggests that these PIFs are indirectly involved in the interaction with the host. As PIF0 has also been shown to be important for ODV binding (Haas-Stapleton et al., 2004), it could be reasoned that the interaction between PIF0, 1 and 2 is important for the binding properties of the ODVs. This would imply that the ODV binding to the epithelium is affected after truncation of PIF1 or 2, while the ODVs of the pif4 deletion mutant would still be able to bind. Alternatively, if PIF1 and 2 are directly involved in ODV binding, the midgut interacting sections might only be exposed in the right conformation when the entry complex is formed. It even seems likely that amino acids from a couple of complex components should be in a proper mutual conformation to make binding happen.

To determine whether the loosely associated PIFs bind to the core independently from each other or whether this requires the presence of the other PIFs as well, we also analysed the binding of PIF0 and 8 to the core in a pif6 deletion mutant by immunoprecipitation of PIF1. This showed that PIF6 is redundant for the binding of PIF0, just as observed with PIF4 (Fig. 8c), while PIF8 was not able bind to the core in absence of PIF6. PIF0 possibly associates only when PIF1-4 are bound together in the stable core, as previous protein interaction studies with yeast two-hybrid ( $\mathrm{Y} 2 \mathrm{H})$ and bimolecular fluorescence complementation assays indicated that PIF0 does not directly bind to one of the core components in monomeric form (Peng et al., 2010b, Zheng et al., 2017). The association of PIF8 on the other hand did not only require the presence of a core complex, but also the presence of PIF0 and 6 (Fig. 8d). Our results show that PIF0 and 6 are essential for formation of the entry complex and that the ZF-domain of PIF8 is essential for association with the complex, just as shown in a Y2H study (Zheng et al., 2017). 
Although the formation of the entry complex was abolished after C-terminal truncation of PIF1 or 2, low mortality levels were observed after oral inoculation of S. exigua larvae with OBs of some of the mutant viruses. This has also been observed in studies with other pif mutant viruses, which are now known to be defective in formation of the entry complex (Pijlman et al., 2003, Ohkawa et al., 2005, Zhu et al., 2013, Dong et al., 2014, Wang et al., 2019). The oral infectivity of the entry complex-defective PIF8 $\triangle$ ZF-mutant of AcMNPV was even shown to be partially rescued when the larvae were first treated with calcofluor white, an agent that damages the peritrophic membrane (Zhu et al., 2013). This has not been observed when ODVs lack either PIF0, 1, 2, 3 or 7 (Song et al., 2008, Liu et al., 2016). Whether the treatment with calcofluor white also rescues the oral infectivity of pif4-, pif5-, pif6 and pif9 deletion mutant viruses remains to be established. Apparently, the ODVs of complex-defective mutants can still be infectious at a low level, indicating that the remaining PIFs, although in suboptimal configuration, remain their functionality to some extent. Another explanation could be that the entry complex is not fully responsible for midgut cell entry and that other ODV envelope proteins can partially substitute the function of this complex when it is dissociated. PIF5 for example, which is not a constituent of the ODV entry complex (Peng et al., 2012), is also known to be important for the infectivity of ODVs via an unknown mechanism (Harrison et al., 2010, Sparks et al., 2011a). Furthermore, at least four other ODV envelope proteins, AC46 (ODVE66) (Sparks et al., 2011b, Xiang et al., 2011), AC111 (Li et al., 2018), AC145 and AC150 (Lapointe et al., 2004, Zhang et al., 2005), have also been shown to be involved in ODV oral infectivity. The viral glycoprotein GP37, that was previously known to be involved in passage of the peritrophic membrane, was recently also reported to have fusogenic properties (Liu et al., 2019). These observations suggests that midgut infection is more complex than initially thought and that the ODVs apparently require more factors than only the complexed PIFs to be fully infectious.

\section{Material and methods}

Virus, insect cells and Lepidopteran larvae. The pif1- and pif2 deletion bacmids have previously been described by Peng et al., 2010 and have the AcMNPV bacmid bMON14272 as backbone. The HA-tagged PIF8 and the truncation mutant bacmid have been described in Javed et al., 2017. Sf21 cells were cultured in T25 flasks with Graces medium (Fisher N.V.), supplemented with $10 \%$ fetal bovine serum (FBS) and $50 \mu \mathrm{g} / \mathrm{ml}$ gentamycin. Sf 21 suspension cultures were grown in Sf900II medium (Fisher N.V.) supplemented with 5\% FBS and 
$50 \mu \mathrm{g} / \mathrm{ml}$ gentamycin. Spodoptera exigua larvae were reared on artificial diet in a $26^{\circ} \mathrm{C}$ climate room with $40 \%$ humidity and a 16:8 h (light:dark) photoperiod.

Construction of recombinant bacmids. The recombinant bacmids were constructed via the Bacto-Bac system (Invitrogen) with pifl- and pif2 deletion bacmids that were partially or completely repaired with the respective genes. In the truncation mutants, the pifl- or pif2 deletion bacmids were repaired with truncated versions that lacked increasing portions of the 3 'end of the coding sequence. To this end, the required parts of the ORFs proceeded by the putative promoters (150bp upstream of the ATG start codon) were amplified by PCR with Phusion polymerase (Invitrogen). The used primers are listed in Table $\mathbf{2}$ with underlined the NcoI and SphI restriction sites introduced for cloning purposes. The amplicons were cloned into a pJET vector (ThermoFisher) for sequence confirmation. The inserts were then re-cloned via the NcoI and SphI restriction sites into a modified pFastBacDual vector (Invitrogen). In this vector, the p10 promoter was deleted and the ORF of the polyhedrin (polh) gene was inserted downstream of the polh promoter as described by Peng et al., 2010 (pFBDAp10-polh). The resulting plasmids were used to construct the recombinant bacmids via Tn7-mediated transposition, using the Bac-to-Bac system (Invitrogen).

Virus proliferation and purification. Sf21-cells were transfected with the constructed bacmids by using Expres2TR (Expres2ion Biotechnologies) as transfection reagent to generate BVstocks of the recombinant viruses that were amplified by an additional infection round in Sf21 cells. OBs were produced by infection of $80 \mathrm{ml}$ suspension cultures with a density of $1.5 \times 10^{6}$ $\mathrm{Sf} 21$ cells/ml with a m.o.i. of $5 \mathrm{TCID}_{50}$ units/cell. After a week of incubation at $27^{\circ} \mathrm{C}$, the cells were pelleted by centrifugation at $4000 \mathrm{x}$ g for $30 \mathrm{~min}$ and lysed by incubation in $0.1 \%$ SDS for $2 \mathrm{~h}$ at $37^{\circ} \mathrm{C}$ with gentle agitation. For complete lysis, the cells were sonicated for $1 \mathrm{~min}$ at

Table 2: Primers used to amplify 3'truncated versions of pifl and pif2.

\begin{tabular}{ll}
\hline Primer name & Sequence (underlined: NcoI and SphI restriction sites) \\
\hline Ac-pif1-Fw & CATCCATGGAATTGCAAACAAAACCACGTAC \\
Ac-pif1-FULL-Rv & CTAGCATGCTTATACAGAGTAGTTGGGGTAAGTTTC \\
Ac-pif1-V459-Rv & CTAGCATGCTTATACACACCACGAGTTGGTG \\
Ac-pif1-F423-Rv & CTAGCATGCTTAGAAGCATACCGGCTGAGC \\
Ac-pif1-A314-Rv & CTAGCATGCTTAAGCGTTCGCGACGG \\
Ac-pif2-Fw & GAGCCATGGCGCGTATCTGCTGTCTATCAC \\
Ac-pif2-Full-Rv & GAGGCATGCTTAAGATAACTGCCCTCTGACG \\
Ac-pif2-N358-Rv & GAGGCATGCTTAGTTGATGCCGTTGCGC \\
Ac-pif2-P351-Rv & GAGGCATGCTTAAGGGTAGGAGCCGGG \\
\hline
\end{tabular}


7 Watt as verified by light microscopy. The OBs were pelleted by centrifugation at $4000 \mathrm{xg}$ for $30 \mathrm{~min}$ and washed three times with Milli Q water. The OBs were further purified by centrifugation over a 30\%-60\% (w/w) sucrose gradient in a Beckmann SW32 rotor at 90,000 x $\mathrm{g}$ for $1 \mathrm{hr}$ at $4^{\circ} \mathrm{C}$. The band with OBs was withdrawn from the gradient with a Pasteur pipette and subsequently pelleted by centrifugation at $4000 \mathrm{x} \mathrm{g}$ for $30 \mathrm{~min}$.

SDS-PAGE and Blue native-PAGE analysis. ODVs of approximately $3.0 \times 10^{8}$ OBs were isolated and fractionated as previously described (Chapter 4). In brief, the ODVs were released from the OBs by treatment with alkaline DAS buffer $\left(0.1 \mathrm{M} \mathrm{Na}_{2} \mathrm{CO}_{3}, 166 \mathrm{mM} \mathrm{NaCl}\right.$ and 10 mM EDTA, $\mathrm{pH} 10.5$ ) to dissolve the polyhedrin matrix and pelleted by centrifugation at 20,800 $\mathrm{x} g$ for $25 \mathrm{~min}$ at $4^{\circ} \mathrm{C}$. The ODVs were then fractionated by resuspension in $50-100 \mu 1$ extraction buffer (6.25 mM Tris, $37.5 \mathrm{mM} \mathrm{NaCl}, 0.5 \%$ Triton-X100, $\mathrm{pH} 7.2)$ and incubated overnight at $4^{\circ} \mathrm{C}$ with gentle rotation. Next day, the nucleocapsids were pelleted by centrifugation at 20,800 $\mathrm{x} g$ for $20 \mathrm{~min}$ at $4^{\circ} \mathrm{C}$ and the envelope proteins in the supernatant was split and mixed with either 4x Laemmli buffer for SDS-PAGE analysis or 4x BN-PAGE sample buffer (200 mM Bis-Tris, $64 \mathrm{mM} \mathrm{HCl}, 200 \mathrm{mM} \mathrm{NaCl}, 40 \%$ glycerol, 0,004\% Ponceau S, pH 7.2), supplemented with 5\% Coomassie G250 in 1: 50 ratio, for BN-PAGE analysis. Prior to SDS-PAGE analysis, the protein samples were heated at 50 or $95^{\circ} \mathrm{C}$ for 5 minutes and separated in a $12 \%$ SDS-PAGE gel. For BN-PAGE, the proteins were separated in 4-16\% Bis-Tris gradient gels as described in the manual for the NativePAGE ${ }^{\mathrm{TM}}$ Novex $^{\circledR}$ Bis-Tris Gel system (Invitrogen). NativeMark ${ }^{\mathrm{TM}}$ unstained protein ladder (Invitrogen LC0725) was stained with Coomassie and used as marker. Blotting to a PVDF membrane was performed with NuPAGE® Transfer buffer (Invitrogen) according to the manufacturer's protocol.

Co-immunoprecipitation. For CoIP analysis, ODVs were released from 5 x $10^{8}$ OBs and purified and fractionated as described above. However, instead of using the extraction buffer, the $500 \mu \mathrm{l}$ IP buffer ( $25 \mathrm{mM}$ Tris, $150 \mathrm{mM} \mathrm{NaCl}, \mathrm{pH}$ 7.2) containing $0.5 \%$ Triton $\mathrm{X}-100$, was used to fractionate the ODVs by a $2 \mathrm{hr}$ incubation at $4^{\circ} \mathrm{C}$ with gentle rotation. In parallel, $30 \mu \mathrm{l}$ of PIF1 antiserum was mixed with $40 \mu \mathrm{l}$ bed-volume of Protein G agarose beads (Pierce) in $500 \mu \mathrm{l} \mathrm{IP}$ buffer and incubated at $4^{\circ} \mathrm{C}$ for $2 \mathrm{hrs}$. After incubation, the Protein $\mathrm{G}$ agaroseantibody complex was collected by centrifugation at $1000 \mathrm{x}$ g for $2 \mathrm{~min}$ and washed with $1 \mathrm{ml}$ IP buffer. The ODV envelope protein suspension was centrifuged at 20,800 x g for 25 min to pellet the nucleocapsids and $450 \mu 1$ supernatant was mixed with the Protein G agarose-antibody complex and incubated overnight at $4{ }^{\circ} \mathrm{C}$ with gentle rotation. For the HA-tagged PIF8 viruses, 
the ODV envelope was isolated as described above and incubated overnight with anti-HA magnetic beads in IP-buffer (Invitrogen) at $4^{\circ} \mathrm{C}$. The remaining $50 \mu \mathrm{l}$ of the supernatant was kept as input control. Next day, the agarose or magnetic beads were collected and washed for 3 times with $1 \mathrm{ml}$ IP buffer. The captured proteins were eluted in $100 \mu 1$ Laemmli buffer by heating at $95^{\circ} \mathrm{C}$ for $10 \mathrm{~min}$. The samples were separated in $12 \%$ SDS-PAGE gels and analysed by western analysis.

Western blot analysis. Western blot analysis with antisera against various PIF-proteins were used as previously described in Peng et al., 2010. In brief, anti-PIF0- (1:1000 dilution) and antiPIF8 antibodies (1:2000 dilution), both produced in rabbit, and anti-HA (1:2000 dilution, Roche 3F10), anti-PIF1 (1:2000 dilution) and anti-PIF2 (1:1000 dilution) produced in rats, were used as primary antibodies. Goat anti-rabbit (1:2000 dilution; Dako) and goat anti-rat (1:2000 dilution; Sigma A8438), conjugated to alkaline phosphatase, were used as secondary antibodies for detection of the proteins of interest by conversion of NBT-BCIP substrate in a blue-purple coloured precipitant in AP-buffer (0.1 M Tris- $\mathrm{HCl}, 0.1 \mathrm{M} \mathrm{NaCl}, 5 \mathrm{mM} \mathrm{MgCl}, \mathrm{pH} 10.5)$

Bio-assays. S. exigua larvae were orally inoculated with isolated OBs of the recombinant viruses to determine their oral infectivity. To this end, the OBs were suspended in $10 \%$ sucrose at a concentration of $3.0 \times 10^{8} \mathrm{OBs} / \mathrm{ml}$ and fed to starved early L3 S. exigua larvae in a droplet feeding assay. The $10 \%$ sucrose solution was coloured blue with Patent Blue V Sodium salt (Fluka) to be able to determine whether the larvae were properly inoculated by looking at their colour. The larvae were allowed to feed on the OB-containing sucrose suspension for $10 \mathrm{~min}$ and were then transferred to a well in a 12-wells plate with a block of artificial diet, and incubated at $27^{\circ} \mathrm{C}$. As the larvae were expected to die at the $4^{\text {th }}$ dpi, the larvae were checked 3 dpi to exclude the larvae that died for other reasons than viral infection. The liquefied larvae were analysed by PCR to validate whether the larva died due to infection with the mutant virus. Therefore, the cadavers were suspended in sterile Milli Q, treated with alkaline (DAS) buffer to dissolve the $\mathrm{OBs}$, and the released ODVs were incubated at $95^{\circ} \mathrm{C}$ for $10 \mathrm{~min}$ to release the viral DNA. The viral DNA was analysed by PCR with GoTaq polymerase (Promega), according to the manufacturers protocol with the primers enlisted in Table 2.

\section{Acknowledgements}

We like to thank Fabiola D. Ortega Murillo and Alexander Sminia, who participated in this project as MSc. students, for the construction of the bacmids to generate the truncation mutants and initial protein and functional analyses. Els Roode and Hanke Bloksma are acknowledged 
for technical assistance in the lab and for insect rearing and cell culturing. This work was supported by grant 824.14.16 of the Netherlands Organization for Scientific Research (NWO). 


\section{Supplementary data}

Multiple alignment of PIF1

CLUSTAL $0(1.2 .4)$ multiple sequence alignment

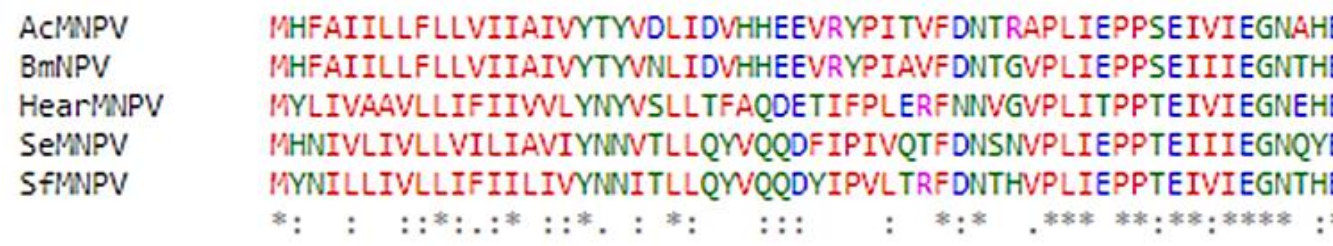

ACMNPV

BmNPV HearMNPV

SeMNPV

SFINPV

ACMNPV

BmNPV

HearMNPV

SelMPV

SFMNPV

CHKTLTPCFTHGDCDLCREGLANCQLFDEDTIVKMRGD-DGQEHETLIRAGEAYCLALDR

CHKTLTPCSTHSDCNLCREGLANCQLFDEDTIVKMRGD-DGQEQEKLIRAGEAYCLALDR

CHKQLTPCQTHLDCDICREGLANCQYFDEKAIITITDAETNTEQTFTIEAGESYCLALDR

CHKQLTPCSTHQOCDICREGLANCQYFDDKTLITITHD-DGEKQEFTIEPGESYCMALDR

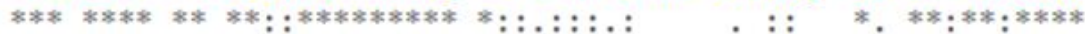

ERARSCNPNTGWLLAETETGFALLCNCLRPGLVTQLNIYEDCNVPVGCAPHGRIDNINS

ACMNPV

BmNPV

HearMNPV

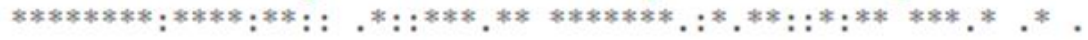

SeMNPV

ASIRCVCDDGYSDYNADTETPYCRPRTVRDVMDESFFPRAPCADGQVRLDHPALNDFY

ASIRCVCDDGYSDYNADTETPYCRPRTVRDWHDESFFPRAPCADGQVRLDHPGLNDFY

SPIURCACDTGFVPDFNVETOTPFCRSRRVRDNIIENTDAFPVAPCERGYIRLDHTGLKPFY RPIIRCSCEVGYVADFNADTQTPFCRTRRIRDVIQNSEFFPHAPCPLGYIPIEHPGLDPEY

SFMNP

RPIIRCSCEVGYVADFNTETQTPYCRTRRIRDVIQNPDFFPLAPCSWPYIPIEHPGLDPAY

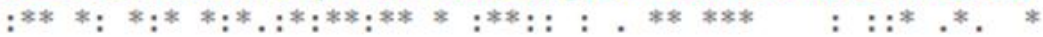

ACINPV

BmNPV

HearMNPV

RRHFRLEDICVIDPCSVDPISGQRTSGRLFHQPTVN-GV-GINGCNCPADDGLLPVFNRH

RRHFRLEDICVIDPCSVDPISGQRTRGRLFHHPIDK-GVNGINGCNCPVSDLLLPVFNRH

NTNIGIPDLCVIDPCSVDPISGERHSGYLQTYRLNFE - - DYHFCVCPIADGLFGVYNDO

SFMNPV

ACMNPV

BmNPV

HearmNPV

LRITTNARNVCVIDPCSVDPVSGQRHEGYLVSYTYEDR -- - ERHFCNCPVDDGLFGVYSDR

296

LQSTNARNACVIDPCTVDPITGQQWGWILVTRYLNDEDKDTQFFCNCSAGHNLFGVYNDQ

TADTGMVRQSDRTVANACLQPFNVHYLSLRHVDYKFFWGRSDHTEFADADIVFQANVNQL

TAETGMIRQSDRTVANACLRPFSVHYLRLRRVDYKYFWGRRDHTEFSDADWFQANANQL

- - LNMIRPSPRQVTNACLKPFNVHISALRRIDYKFFWAHTDRTR-SDEDVIAAVQESQL

-. - PTPNRPSSERLVNACLKPFNVHISAIPVIEYKWFWGHIDLFV-SDDDIVAFVDRNRV

- - PNVIIRPSAEKLVNACIQPFNVHVAQLPAIEYKWFWGQRNLYT-SDDDVVATVRPDQI

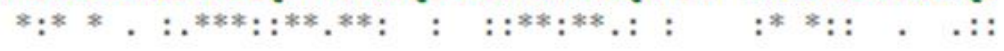

ACMNPV

BmNPV

HearmNPV

SelWPV

SFINPV

SHERYRAILYPLLESHPDVTEIVTVNMGVMKISVSYDTTLKNILLPSSVFRLFR--FKES

The multiple alignments continues on the next page. 


\begin{tabular}{|c|c|c|}
\hline AClNPV & GTAQPVCFFPGV-GRCITVNSDSCIRRHAGGQVITAETFTNSWCVLSREGTHIKWWSRAS & 474 \\
\hline BmNPV & STSRSSCFFPGV-GRCILADPDACIRRHGGFFUNTAETFTNSWCILSREDTHIKIWSSAS & 471 \\
\hline HearmNPV & IRTQEPCFFPGHEGRCITHNPHLCIRRHANFQVGTAEFFTNYWCYLSRDEGIIQIWSPPD & 471 \\
\hline SelMNPV & --NYTLCFYPGLEGRCIVNGYYNCIRRHGVVQVNTAERFSDKQCILSRDGRWIRWWHQPQ & 466 \\
\hline \multirow[t]{2}{*}{ SFMNPV } & H-RSATCFYPGF-GRCWHDHTNCIRRFGSVQVGTAENLKGTQCYLSRDRWWIRIWYKPQ & 469 \\
\hline & $* *: * * * * *: . \quad * * * * \ldots \quad * * * *: \ldots * * * *: \quad *:: *$. & \\
\hline ACINPV & RYPRGDAPAALRLRGFFL --NNDRERNTIRAVTTGDMT--QGQQIDALTQILETYPNYSV & 530 \\
\hline BmNPV & RYPRGDAPAVLRLRDFFL --DDRREQNTIRTITTGANT--QGQQIDALTQILQTYPNYSL & 527 \\
\hline HearmNPV & RYRAEEFPVALRNRLLWAIDNNDRTNNTINIVFSREALPTH---RNEVRDILKTYANYSV & 528 \\
\hline SelMPV & WYRSGRFPVALWSVKFVYSG-DRDHTTVRPVFGIDTTAEYADIWNLLIPLLRTYHHISI & 525 \\
\hline \multirow[t]{2}{*}{ SFINPV } & WYTNRRYAVALYNGLFFVIN-TRDFRTVRFVSATDLLINQNDLNNSLITLLNTYHHISV & 528 \\
\hline & $* \quad .^{*} \quad: \quad 0^{*} \quad *::: \quad:::^{*},{ }^{*}:{ }^{*}:$ & \\
\hline ACINPV & -530 & \\
\hline BmNPV & -527 & \\
\hline HearmwPV & T 529 & \\
\hline Sel'NPV & Q 526 & \\
\hline SFMNPV & V 529 & \\
\hline
\end{tabular}

Fig. S1 Multiple alignment of PIF from various alphabaculoviruses. PIF1 of AcMNPV E2 strain (Genbank AIU56976.1) was aligned with its homologues in BmNPV (NP_047516.1), HearMNPV (ACH88565.1), SeMNPV (NP_037796.1) and SfMNPV (YP_001036327.1). The asterisks (*) indicate the completely conserved amino acid residues, the colon (:) indicates the conservation of amino acid residues with strong similarity in properties (scoring > 0.5 in Gonnet PAM 250 matrix) and the dots (.) indicate the conservation of residues of amino acids with weak similarity in properties (scoring $<0.5$ in Gonnet PAM 250 matrix). These alignments show that the C-terminal part of PIF1 is less conserved than the $\mathrm{N}$-terminal part. 
Multiple alignment PIF2

CLUSTAL O(1.2.4) multiple sequence alignment

\begin{tabular}{|c|c|}
\hline ACMNPV & $\ldots$ MYRVLIVFFLFVFLYIVYQPFYQAYLHIGHA \\
\hline BmNPV & MYRXLIWFLFVFLYIVYQPFYQAYLHIKRA \\
\hline Hearl'NPV & MLIVILLLFVLLVIFLYVLYRPIUHLAWRLMYKA \\
\hline SelNPV & MPANPSIANATPTTRVRIERAIQLSATAAMFLLLMIVCWIFLFLLCKPIYDAHLEIKKS \\
\hline \multirow[t]{2}{*}{ SFMNPV } & MIVTIERAIQLSAT-WMFLLLLIVCVILFLFLLCKPIIYDAHAQIKKS \\
\hline & ${ }^{*}: \ldots::^{* *}:::::^{*}:: * \quad:::$ \\
\hline AClNPV & QQDYNDTLDDRMDYIESWMRRRHWPIEALPAIRFDTNLGTLAGDTIKCMSVPLFVSDID \\
\hline BmNPV & QQKYNDTLDDRMDYIESWMRRRRWPIEALPTIRFDTNLGTLAGDTIKCMSVPLFVSEID \\
\hline HearlwPV & QREYNETIDDRIDYYQEVLRRRQWPLHSLPNINFNTNLGTINDGELKCLSVPVFVGPVE \\
\hline SeMNPV & QTDYNETVDERIDYMRNVLQRRRFVPLSALPNIVFNTNLGTINEGESKCLSVPIWGFSN \\
\hline \multirow[t]{2}{*}{ SFMNPV } & QKAYNETVDERIYYMQNVLQRRRFVPLSALPNIIFNTNLGTINEGEQKCLSVPIYGFGN \\
\hline & ${ }^{*}:^{*}::^{*}:{ }^{*}: .^{*}::^{* *}::^{* *}::^{* *} * *:^{* * * * *}:{ }^{* *}: * * *::^{*}, \quad:$ \\
\hline ACMNPV & LPIIFDCSQICDNPSAAYFFVNETDVFWNGHRLTVGGYCSTNSLPRNCNRETSVILMSLN \\
\hline$B m N P V$ & IPIIFDCSOVCDNPSAAYFFVNNTDVFVNNGHKLTVGGYCSTNSLPRNCNRETSVILMSLN \\
\hline HearlMNPV & TPNFDCTETCDNPSAFYFFVGEYDKFWNGELLDRGGYCTTNSIPRNCNRETSVILHGLN \\
\hline SEMNPV & TPNFDCTVLCDNPAAVYFWDEYDKFVINGONLLRGGYCTTSSVPRNCNRETSVIIHSLN \\
\hline \multirow[t]{2}{*}{ SFMNV } & TPNFDCAALCDNPTASYFWNQYDKFVINGQVILTQGGYCTTSSVPRNCNRETSWVLHSLN \\
\hline & $* * * *: \quad * * * *:^{*} * *:^{*},{ }^{*} * *:^{* *},{ }^{*} \quad * * * *:^{*} .^{*}: * * * * * * * * *:: .^{* *}$ \\
\hline ACMNPV & QUTCIAEDPRYYAGTDNMITQLAGRQHFDRIMPGQSDRNVLFDRLLGREVNVTTNTFRRSW \\
\hline BmNPV & QUTCIAEDPRYYAGTDNMITQIAGRQHFDRIMPGQSNRNILFDRLLGREVNVATNTFRRSW \\
\hline HearlwPV & QUTCIAEDPRYFAGPQNMSQVAGRQHADRIFPGQIGRNILFDRLLGTEVDVSRNTFRSHWW \\
\hline SelMNPV & QUSCIAEDPRYFSGIQNINQVAGRQHIDRIAPGQANRNVLFDRLLGVEVDVTRNTFRSHWW \\
\hline \multirow[t]{2}{*}{ SFMNPV } & QUTCIAEDPRYFSGPQNINQVAGRQHIDRIAPGQANRNVLFDRLLGVEVDVTRNTFRSHWW \\
\hline & $* *: * * * * * * * *:^{*}:^{*}: .^{*}: * * * * * * * * * * * \quad .^{* *}: * * * * * * * * * *: * * * * \quad *$ \\
\hline ACMNPV & DELLEDGT-RRFEMRCNARDNNNNLMFVNPLNPLECLPNVCTNVSNVHTSVRPVFETGEC \\
\hline BmNPV & DELLEDGT-RRFEMRCNARDNNNNLMFVNPLNPLECLPNCTSSVRNVHTSVRPVFETGEC \\
\hline HearlwPV & DELLPDGT-RRFEMRCNALDDHENRMFLNPLNPIECLPNVCTNVRRVALSVRPNFSTGEC \\
\hline SelNPV & DEEMPDGSGRRFEMRCNALDDHNNRMFVNPFNPIECLPNVCTNVNFVMIPOVRPNFETGEC \\
\hline \multirow[t]{2}{*}{ SFMNPV } & DEEMPDGSGRRFEMRCNALDDHNNRMFVNPFNPIECLPNICTNVNFVSPDVRPNFETGEC \\
\hline & $* *: * *: * * * * * * * * * *:::^{*} * *: * *: * *: * * * * *: * *, * \quad * \quad, * * * *, * * * *$ \\
\hline AclMNP & DCGDEAVTRVTHIVPGDRTSMCASIIDGLDKSTASYRYRVECVNLYTSILNYSNNKLLCP \\
\hline BmNPV & DCGDEAVTRVTHIVPGDRTSMCASIVDGLDKNTASYRYRVECVNLYTSILNYSSNKLLCP \\
\hline HearlMNV & ECGDVNETRVTHIVPGDKTSMCAAWVRFNRDLMSHQLRVDCITRDMPPISKWHKDMILCP \\
\hline SelNPV & ECGDFEVTRVRHLDPDDKTSMCAAVVDTFDERTLSLQYRVDCIMLDMPVEKYDRNYLWICP \\
\hline \multirow[t]{2}{*}{ SFMNPV } & ECGDFAVTRVRHVDPNDRTSLCAAVTDTYDERTRSLQYRVECMNMNPVEKYRRNMLWCP \\
\hline & 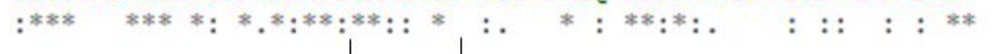 \\
\hline ACIMNPV & SDTFOSNTDAAFAFEVPGSYPLSRNGINEPTYRFYLDTRSRVNYNDVRGQLS- \\
\hline BMNNPV & SNNFDSNTDAAFAFEVPGSYPLSGNGLNEPTYRFYLDTRSRVRYNDVRGLLS- \\
\hline HearlMNV & PDVFVQNSDNAFYFTLPGSFPISNTGVEPTYRFYMQTRNRVNYIRRDLPS- \\
\hline SEMNPV & SNIFNQNTDNAFLFTVPGSFPISGNGIDEPTURLYMEVRNRIEFNVERPRPPV \\
\hline SFMNPV & SNIFTTNTDNAFLFTVPGSFPTSGNGLDEPTWRFMMEVQSRIDFGQIRASPPV \\
\hline
\end{tabular}

Fig. S2 Multiple alignment of PIF from various alphabaculoviruses. PIF2 of AcMNPV E2 strain (Genbank AIU57013.1) was aligned with its homologues in BmNPV (AIS92745.1), HearMNPV (QBM79106.1), SeMNPV (CDG72376.1) and SfMNPV (YP_001036326.1). The asterisks (*) indicate the completely conserved amino acid residues, the colon (:) indicates the conservation of amino acid residues with strong similarity in properties (scoring > 0.5 in Gonnet PAM 250 matrix) and the dots (.) indicate the conservation of residues of amino acids with weak similarity in properties (scoring $<0.5$ in Gonnet PAM 250 matrix). These alignments show that the C-terminal part of PIF2 is less conserved than the N-terminal part. 


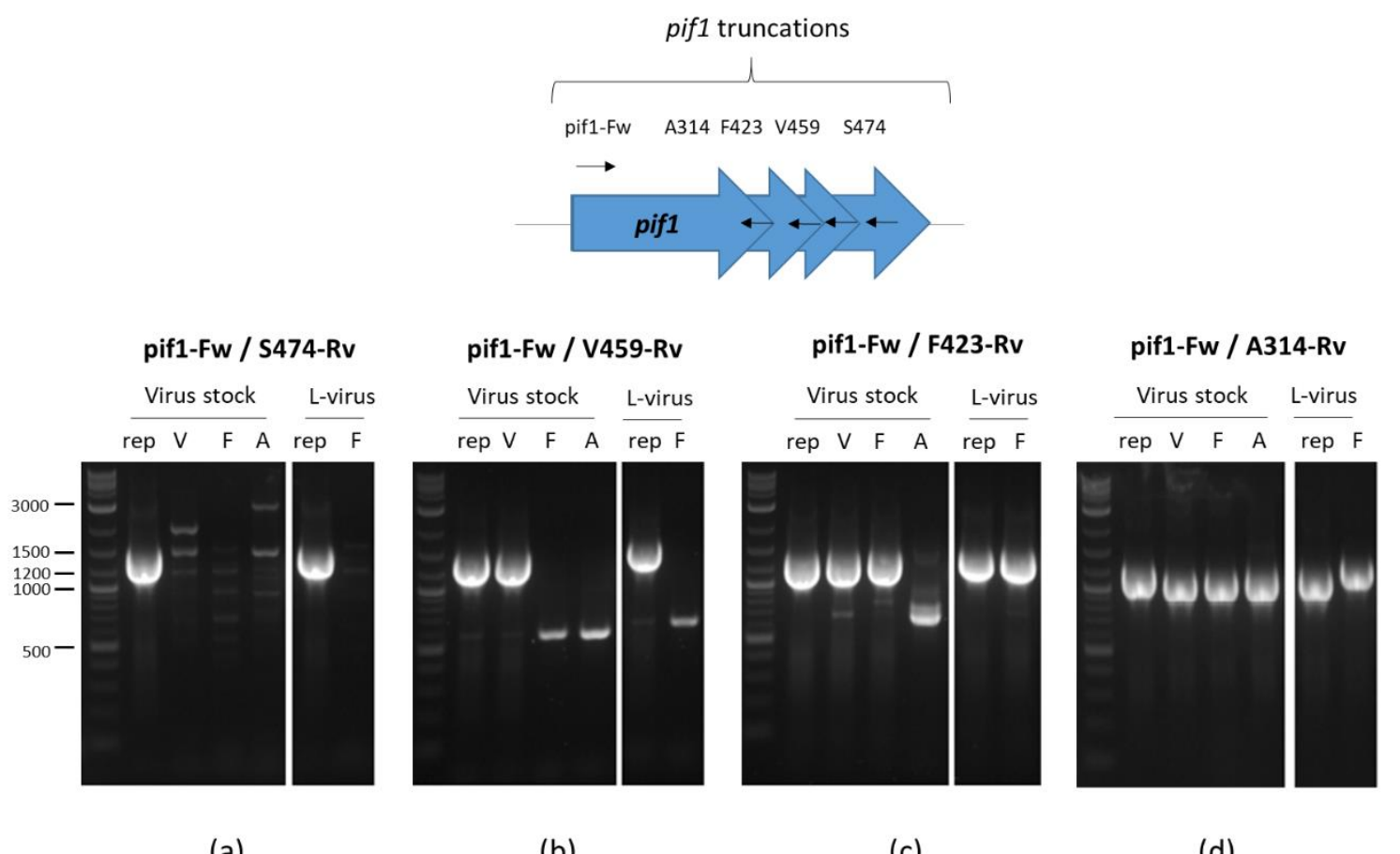

(a)

(b)

(c)

(d)

Fig. S3: PCR analysis of ODV-derived bacmid from the pifl virus stocks that were used to inoculate the larvae (virus stock, left panels) and the bacmids that were retrieved from infected larvae (L-virus, right panels) from the bioassay. On top, a schematic overview of the annealing sites of the various primers. The mutants are indicated by the amino acid at which the PIF1 protein was truncated (V, F or A). This analysis was performed with four different primer sets in which the reverse primer was varied: S474-Rv (a), V459-Rv (b), F423-Rv (c) and A314-Rv (d).

When using the reverse primer pifl-S474-Rv that annealed downstream from the first truncation site, no specific amplicon was produced for any of the truncation mutants, as expected. This was first shown for the virus stocks that were used to inoculate the larvae, indicating that these stocks did not contain wild type or full length repair viruses (a). PCR amplification on the virus that was retrieved from the single liquefied larva (L-virus) after inoculation with the pifl-F423 truncation mutant, only produced an amplicon with reverse primers that annealed at the respective truncation sites or more upstream (F423-Rv and A314$\mathrm{Rv}$ ) (c, d), but not with primers that annealed downstream of the truncation sites (S474-Rv and V459-Rv) (a, b). This confirms that the liquefied larvae was indeed infected by the pifl-F423 truncation mutant. 


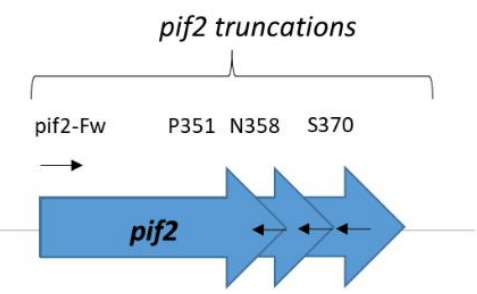

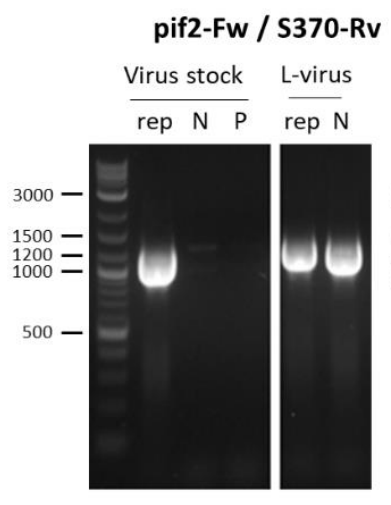

(a)

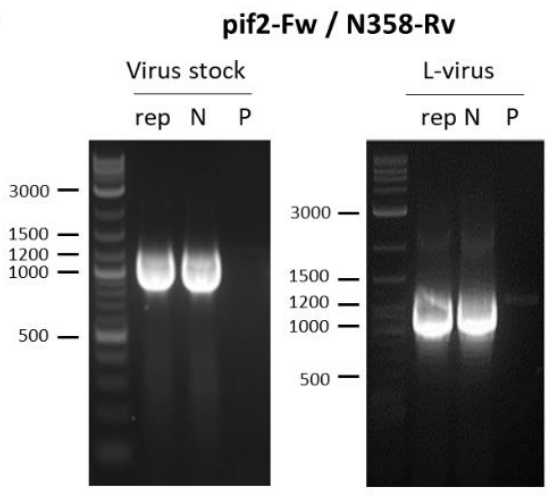

(b)

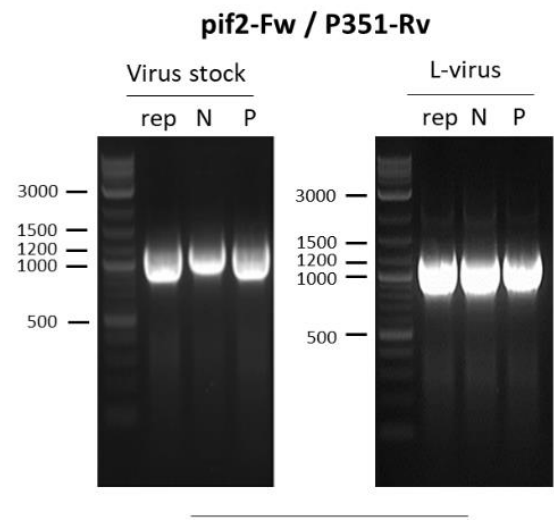

(c)

Fig. S4: PCR analysis of ODV derived bacmid from the pif2 virus stocks that was used to inoculate the larvae (virus stock, left panels) and the bacmid that was retrieved from infected larvae (L-virus, right panels) of the bioassay. On top, a schematic overview of the annealing sites of the various primers. The mutants are indicated by the amino acid at which the PIF2 protein was truncated $(\mathrm{N}$ or $\mathrm{P})$. This analysis was performed with four different primer sets in which the reverse primer (Rv) was varied: S370-Rv (a), N358-Rv (b), P351-Rv (c).

When using the reverse primer pif2-S370-Rv that annealed downstream from the first truncation site, no specific amplicon was produced for any of the truncation mutants of the virus stocks, indicating that these are clean. However, an amplicon with this reverse primer was produced with the virus that was retrieved from the larvae that were inoculated with the pif2N358 truncation mutants, indicating that those larvae were infected by wild type or full repair virus. PCR analysis on the virus that was retrieved from the single liquefied larva that was inoculated with the pif2-P351 only produced an amplicon when using the P351-Rv primer (c) and not with N358-Rv (b). This confirms that the liquefied larvae was indeed infected by the pif1-F423 truncation mutant. 
-100 - 


\title{
Chapter 7
}

\section{Reconstruction of the interactome of the baculovirus per os infectivity factors}

Bob Boogaard, Jan W. M. van Lent, Monique M. van Oers

\begin{abstract}
The baculovirus occlusion-derived virus (ODV) phenotype infects the midgut epithelium of insect larvae. The ODV-envelope contains at least ten different proteins that are required for this oral infection and are named per os infectivity factors (PIFs). Nine of these PIFs, PIF0 to 4 and PIF6 to 9, form the ODV entry complex. PIF5 is the only PIF that is not a constituent of this complex, suggesting that the entry complex is not the only determinant of ODV per os infectivity. Moreover, there are also other ODV-envelope proteins that influence the infectivity of the ODVs, suggesting that the entry complex function in relation with other proteins. We reconstructed a PIF interactome for the Autographa californica multiple nucleopolyhedrovirus by identification of the interaction partners of PIF5, 6, 8 and 9 using immunoprecipitation of these PIFs followed by mass spectrometry analysis. Our analysis revealed that the PIFs interact not only with other PIF-proteins, but also with other ODV proteins that are involved in nucleocapsid assembly, virion production and OB-formation. Especially PIF5 and 9 were found to have many non-PIF interaction partners. Furthermore, PIF0 and 1 were the only components of the complex that co-precipitated with PIF5, suggesting that these two PIFs also occur outside the complex. These results confirm the idea that the entry complex acts in concert with other viral proteins.
\end{abstract}




\section{Introduction}

The Baculoviridae is a family of large DNA viruses that infect their insect hosts at the larval stage via the oral route (reviewed by Williams et al., 2017, Harrison et al., 2019). Two different virion phenotypes are produced during the replication cycle: budded viruses (BVs) and occlusion-derived viruses (ODVs). The BVs are formed when the newly formed nucleocapsids migrate towards the host cell plasma membrane and bud off to spread the viral infection systemically within a host. The ODVs are formed later in the replication cycle, when the nucleocapsids are retained inside the nucleus and are enveloped by derivatives of the (inner) nuclear membrane (Braunagel and Summers, 2007, Shi et al., 2015). In all viruses, except the ones belonging to the genus Betabaculovirus, the ODVs are embedded in a matrix of crystallized polyhedrin protein, which results in the formation of polyhedral-shaped occlusion bodies (OBs) in the nucleus. Hence, the name nucleopolyhedrovirus (NPVs). In betabaculoviruses, the ODVs are embedded in a protein matrix of granulin. The protein matrix of the OBs protects the ODVs outside the insect host upon dispersal of the virus into the environment. The ODVs are responsible for the horizontal transmission of the virus from one larva to the next.

Upon ingestion of contaminated plant material, the OBs dissolve in the highly alkaline environment of the midgut lumen. The released ODVs pass the peritrophic membrane and infect the midgut epithelial cells by direct fusion of the viral envelope with the plasma membrane (Horton and Burrand, 1993) of the microvilli by which the nucleocapsids are deposited into the host cell. The ODV-envelope contains at least ten different proteins that are crucial for the infection of the midgut epithelium, the so-called per os infectivity factors (PIFs). Per definition, these proteins are only involved in the primary infection of the midgut epithelium by ODVs and not in systemic spread of the virus infection by BVs (Faulkner et al., 1997, Kikhno et al., 2002, Pijlman et al., 2003, Ohkawa et al., 2005, Fang et al., 2009, Sparks et al., 2011, Nie et al., 2012, Zhu et al., 2013, Javed et al., 2017, Liu et al., 2016, Wang et al., 2019, Chapter 4).

Nine PIFs form a large complex in the ODV-envelope, the so-called ODV entry complex, that consists of a stable core, formed by PIF1 to 4, to which PIF0 and PIF6 to 9 associate more loosely (Peng et al., 2010, 2012, Chapter 2, 3, 4). PIF5 is the only PIF that is not a constituent of this complex (Peng et al., 2012). The core is very stable as it was still found in western blots after partial denaturation of ODVs in SDS-solution at $75^{\circ} \mathrm{C}($ Chapter 3). The loosely associated components apparently dissociated under these conditions. The complete entry complex can 
only be observed under non-denaturing conditions and is detected as a $480 \mathrm{kDa}$ band in blue native-PAGE gels (Peng et al., 2012).

ODVs do not only rely on the entry complex to be fully infectious. For example, the oral infectivity of mutant ODVs that lack PIF5 is significantly reduced, while the entry complex is still formed (Harrison et al., 2010, Sparks et al., 2011a, Peng et al., 2012). Furthermore, the entry complex is important, but not indispensable for midgut infection as various pif mutants were incidentally able to infect the larvae (Ohkawa et al., 2005, Fang et al., 2009, Dong et al., 2014, Zhu et al., 2013, Wang et al., 2019), which are now known not be able to form the complex (Peng et al., 2012, Wang et al., 2019, Chapter 4). The infectivity of a complexdeficient pif8 mutant, that lacks the zinc finger (ZF-) domain, was partially rescued after damaging the peritrophic membrane with calcofluor white (Zhu et al., 2013). This suggests that the complex (or the zinc finger) is involved in passing the peritrophic membrane and that the mutant virus is able to infect the midgut cells in absence of the entry complex. Moreover, absence of ODV proteins AC18 (Wang et al., 2007), ODV-E66 (AC46) (Xiang et al., 2011, Sparks et al., 2011b), AC111 (Li et al., 2018), AC114 (Wei et al., 2012), AC124 (Liang et al., 2015), AC145 or AC150 (Lapointe et al., 2004, Zhang et al., 2005) also influences the virus infectivity in vivo. Among these, AC111 appeared important for the infection of Trichuplusia ni larvae, but was dispensable for infection of Spodoptera exigua, adding an extra layer of complexity ( $\mathrm{Li}$ et al., 2018). These observations indicate that the ODV entry complex is a central player for the initiation of midgut infection, but that other viral proteins are also required for optimal infectivity in vivo.

In this chapter, we reconstructed a PIF-interactome using the Autographa californica multiple nucleopolyhedrovirus (AcMNPV) as a model, to better understand the relation between PIFs and other proteins in the ODV. This was done by immunoprecipitation of a panel of HA-tagged PIFs, followed by mass spectrometry to identify the interaction partners of these PIFs

\section{Results and discussion}

\section{Selection of PIF proteins for co-immunoprecipitation experiments}

It was studied how the AcMNPV ODV entry complex interacts with other ODV proteins. We therefore mapped the proteins that co-immunoprecipitated with a panel of HA-tagged PIFs when using anti-HA magnetic beads, by mass spectrometry. PIF1 of the stable core has been analysed before using a similar strategy (Peng et al., 2012). This resulted in the precipitation of all known components of the complex and a component of the polyhedrin matrix, AC5. In this 
study, the loosely associated components entry complex were precipitated as these proteins are probably more likely to interact with other ODV envelope proteins than the PIFs in the stable core. Three loosely associated components, PIF6, 8 and 9, were efficiently immunoprecipitated. PIF0 and PIF7 were excluded from these experiments as PIF0 failed to precipitate efficiently, and PIF7 was not detectable by mass spectrometry despite effective precipitation as shown by western blot analysis (Supplementary data Fig. S1). PIF5, the only PIF that is not a constituent of the complex, was also analysed to obtain first data on the interaction partners of this PIF in the ODV envelope.

PIF5 interacts with two components of the entry complex: PIF0 and 1.

PIF0 and 1 co-precipitated with all four target proteins (PIF5, 6, 8 and 9) and were the only components of the entry complex that co-precipitated with PIF5 (Fig. 1). In the PIF5 precipitation, PIF0 was 80 times enriched over the negative control (wild type virus without an HA-tagged protein) (t-test, $\mathrm{p}=0.003)$ and PIF1 almost 40 times ( $\mathrm{t}$-test, $\mathrm{p}=0.0006$ ) (Supplementary data Fig. S2.1). The interaction between PIF5 and PIF0 was confirmed by western blot analysis of the co-precipitated proteins with PIF0 antiserum. This was not confirmed for PIF1 as this PIF was hard to detect with PIF1 antiserum in the HA-tagged PIF5

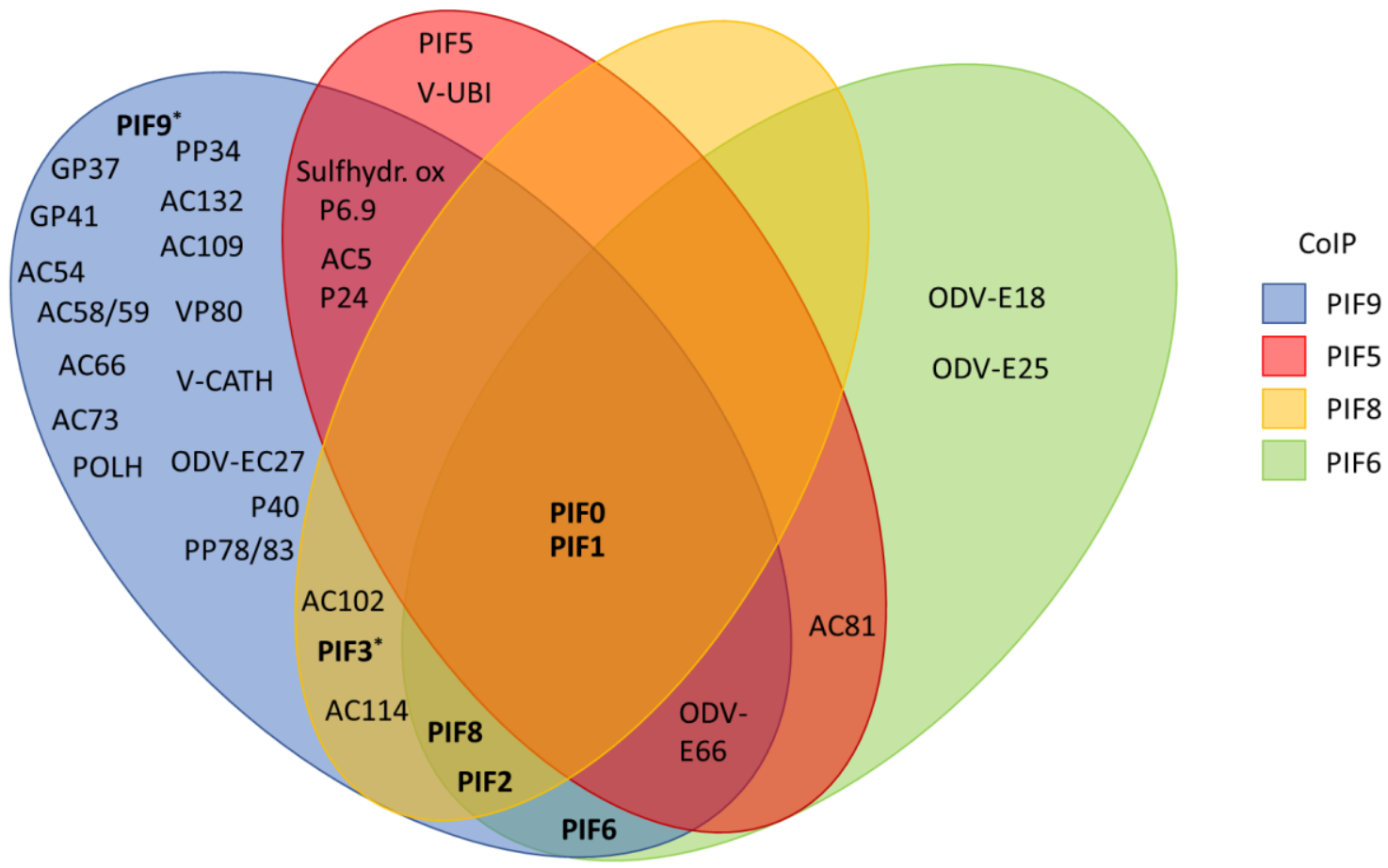

Figure 1: Venn diagram of the identified interaction partners of PIF 9 (in blue), PIF5 (in red), PIF8 (in yellow) and PIF6 (in green). These PIFs were immunoprecipitated in triplicate via the HA-tags with anti-HA magnetic beads and co-precipitated proteins were identified by LC-MS/MS. Wild type ODVs were analysed in parallel as negative control. *PIF3 and 9 were detected by a single peptide after immunoprecipitation of PIF6, for which they were excluded from further statistical analysis. 
repair mutant virus (Supplementary data Fig. S1b). Furthermore, PIF5 was not enriched after immunoprecipitation of one of the other components of the entry complex (PIF6, 8 or 9) (Fig. 1). As immunoprecipitation of PIF5 resulted in the precipitation of only PIF0 and 1, and not of any other component of the entry complex, it is suggested that these two PIFs are not only constituents of the ODV entry complex, but also occur outside the complex. The interaction between PIF5 and PIF0 and 1 had also been detected in in other studies by yeasttwo hybrid (Y2H) assays with PIF homologs of Helicoverpa armigera NPV (HearNPV) (Peng et al., 2010b). However, PIF5 was not identified as a PIF1 interaction partner in the ODVenvelope of AcMNPV in the earlier co-immunoprecipitation study with PIF1 antiserum (Peng et al., 2012). Maybe the usage of PIF1 antiserum was not suitable for detection of this interaction or this interaction can only be detected in one direction as with the $\mathrm{Y} 2 \mathrm{H}$ assays. The Y2H assays also suggested an interaction between PIF5 and PIF3, but our data do not provide any evidence for that.

The biological importance of the putative interaction between PIF0, 1 and 5 is unclear as mutant viruses that lack one of these PIFs have different phenotypes. PIF0 and 1 are involved in the binding of ODVs with the midgut epithelium, while PIF5 appeared dispensable for ODV binding (Haas-Stapleton et al., 2004 Ohkawa et al., 2005, Sparks et al., 2011). This suggests that PIF0 and 1 in addition to ODV binding have other functions as well during midgut infection.

Smaller sized components of the entry complex are more difficult to detect by mass spectrometry.

PIF2 and 8 precipitated together with the components of the entry complex (PIF6, 8 and 9) and not with PIF5 (Fig. 1). The smaller sized PIF3 (27 kDa), PIF6 (15 kDa) and PIF9 (17 kDa), were not always detected after immunoprecipitation of one of the target proteins. PIF3 was only detected after precipitation of PIF8 and 9, PIF6 only with PIF9, and PIF9 only after direct precipitation with the anti-HA magnetic beads (Fig. 1). This might be due to the fact that less peptides are formed by these smaller PIFs upon trypsin digestion and are therefore more difficult to be detected by mass spectrometry in complex peptide mixtures, especially when these PIFs are present at low quantities (Pers. comm. Sjef Boeren). Precipitation of PIF6 or 9 nevertheless resulted in the precipitation of PIF0, 1, 2 and 8, confirming their presence in the entry complex. 
ODV-E66 interacts with PIF6 and 9 of the entry complex and PIF5.

Of the non-PIF proteins, ODV-E66 was identified most often as a PIF interaction partner. This protein was found after immunoprecipitation of three of the four target proteins: PIF5, 6 and 9 (Fig. 1 and 2). Other studies also indicated that this protein closely associates with components of the entry complex. $\mathrm{Y} 2 \mathrm{H}$ and bimolecular fluorescence protein complementation (BiFC) assays indicated that ODV-E66 interacts with PIF2, 3 and 4 (Peng et al., 2010b, Dong et al., 2014). However, this protein was not identified as a PIF1 interaction partner in an earlier coimmunoprecipitation study (Peng et al., 2012) and appeared not to be a constituent of the ODV entry complex, when the ODV-envelope was analysed under native conditions (Wang et al., 2019). The interaction between ODV-E66 and PIF5 was previously also identified with Y2H assays (Peng et al., 2010b). Our study confirms the close association of ODV-E66 with the PIFs in the ODV envelope and is in accordance with studies that showed that ODV-E66 is indeed important for the oral infectivity of ODVs. This protein is probably involved in ODV binding as peptide derivatives of this protein have affinity for the midgut epithelium (Sparks et al., 2011b, Xiang et al., 2011) (See table 1 for further information). This protein is was recently also shown to be important for the degradation of the peritrophic membrane (Hou et al., 2019).

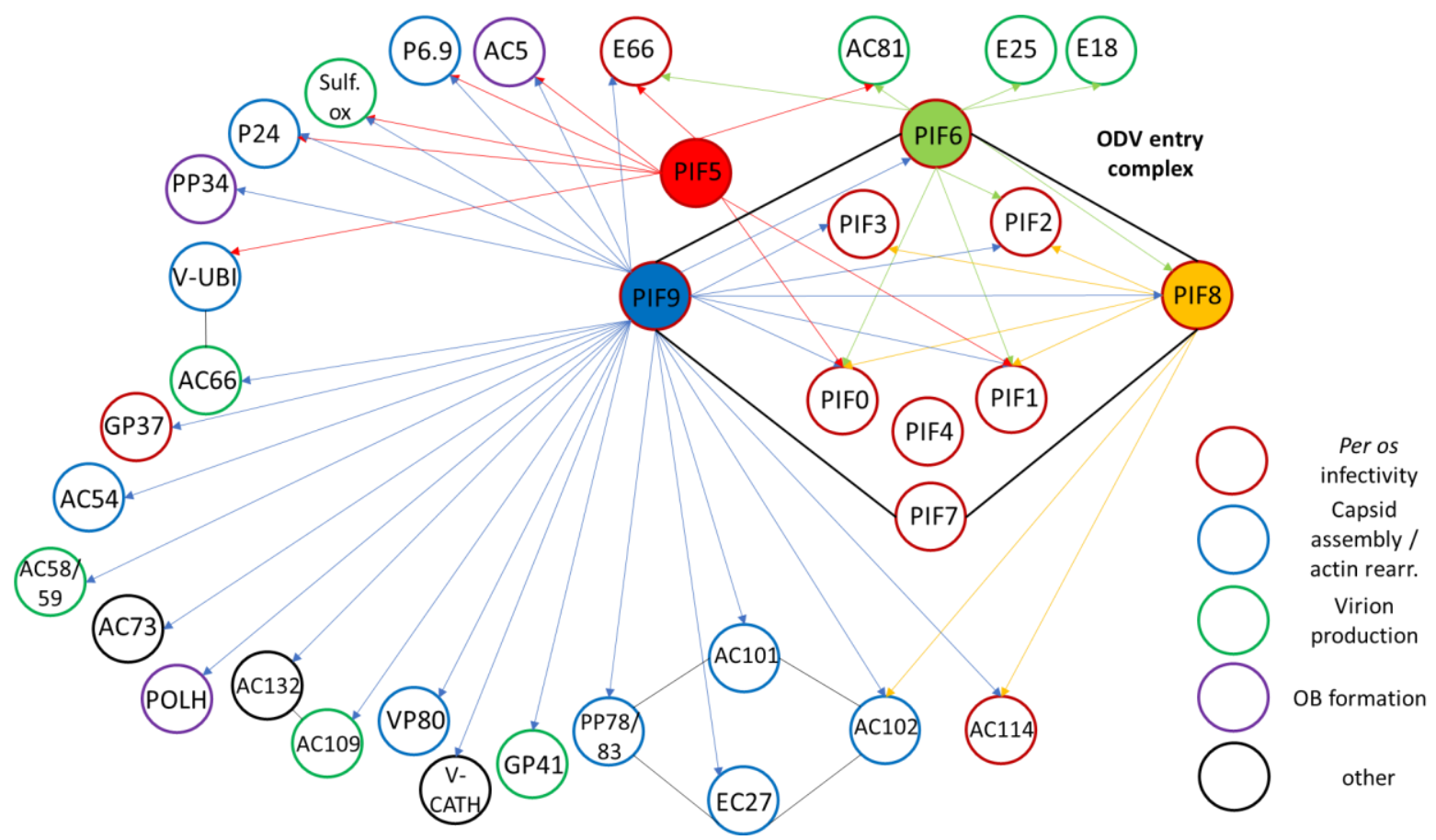

Figure 2: A protein interaction network based on the identified interaction partners after immunoprecipitation of one of the target proteins; PIF5, 6, 8 and 9. The identified proteins were categorized according to their reported function into five different groups: per os infectivity, encircled with dark red; nucleocapsid assembly and actin rearrangement, encircled with blue; virion production, encircled with dark green; OB formation, encircled with purple; and rest, encircled with black. 
Table 1: Overview of identified PIF interaction partners according to their functions

\begin{tabular}{|c|c|c|c|}
\hline Protein & $\begin{array}{l}\text { AcMNPV } \\
\text { ORF }\end{array}$ & Remarks & References \\
\hline \multicolumn{4}{|c|}{ Per os infectivity } \\
\hline ODV-E66 & $a c 46$ & $\begin{array}{l}\text { Hyaluronidase activity, peptide } \\
\text { derivatives bind midgut epithelium, } \\
\text { degrades the peritrophic membrane. }\end{array}$ & $\begin{array}{l}\text { Sparks et al., } 2011 \mathrm{~b}, \\
\text { Xiang } \text { et al., } 2011 \\
\text { Hou } \text { et al., } 2019\end{array}$ \\
\hline GP37 & ac64 & $\begin{array}{l}\text { Polyhedron and BV associated, binds } \\
\text { chitin, facilitate PM-passage, } \\
\text { fusogenic properties }\end{array}$ & $\begin{array}{l}\text { Vialard } \text { et al., 1990, Li } \text { et al., } \\
\text { 2003, Wang et al., 2010, Liu et } \\
\text { al., 2011, Liu et al., } 2019\end{array}$ \\
\hline $\mathrm{AC} 114$ & ac114 & $\begin{array}{l}\text { Associated with BVs and ODVs; } \\
\text { involved in embedding of ODVs in } \\
\text { protein matrix }\end{array}$ & $\begin{array}{c}\text { Braunagel et al., 2003, } \\
\text { Wang et al., 2010, } \\
\text { Wei et al., } 2012 \\
\end{array}$ \\
\hline \multicolumn{4}{|c|}{$\begin{array}{l}\text { Nucleocapsid assembly / } \\
\text { actin rearrangement }\end{array}$} \\
\hline PP78/83 & $a c 9$ & $\begin{array}{l}\text { Essential gene in Group I and II } \\
\text { lepidopteran NPVs, WASP-like } \\
\text { protein for actin nuclear assembly in } \\
\text { association with Ac101, Ac102 and } \\
\text { ODV-EC27 }\end{array}$ & $\begin{array}{l}\text { Vialard et al., 1993, Russell et } \\
\text { al., 1997, Goley et al., 2006, } \\
\text { Ohkawa et al., } 2010\end{array}$ \\
\hline V-UBI & ac35 & $\begin{array}{l}\text { Might inhibit degradation of short- } \\
\text { lived viral proteins; important for BV } \\
\text { production by facilitating nuclear } \\
\text { egress of nucleocapsids in } \\
\text { association with AC66 and AC } 141\end{array}$ & $\begin{array}{c}\text { Haas et al., 1996, Biwas et al., } \\
2018\end{array}$ \\
\hline AC54 & $a c 54$ & $\begin{array}{l}\text { Core gene, associated with BVs and } \\
\text { ODVs, Nucleocapsid assembly }\end{array}$ & $\begin{array}{l}\text { Olszweski et al., 1997a, } \\
\text { Guan } \text { et al., } 2016\end{array}$ \\
\hline P6.9 & ac100 & $\begin{array}{l}\text { Core gene, DNA binding protein, } \\
\text { nucleocapsid assembly }\end{array}$ & $\begin{array}{l}\text { Wang et al., 2010b, } \\
\text { Liu et al., 2012, Lai et al., } 2018\end{array}$ \\
\hline $\begin{array}{l}\mathrm{AC} 101 / \\
\mathrm{C} 42\end{array}$ & ac101 & $\begin{array}{l}\text { Core gene, capsid associated, form a } \\
\text { complex with AC102, PP78/83 and } \\
\text { ODV-EC27, regulated by miR-3 }\end{array}$ & $\begin{array}{l}\text { Braunagel et al., 2001, } \\
\text { Garavaglia et al., 2012, Hepp et } \\
\text { al., 2018, Jiao et al., } 2019\end{array}$ \\
\hline AC102 & ac102 & $\begin{array}{c}\text { Associated with the nucleocapsids, } \\
\text { involved in nuclearization of G-actin } \\
\text { with AC101, PP78/83 and } \\
\text { ODV-EC27 }\end{array}$ & Hepp et al., 2018 \\
\hline VP80 & ac104 & $\begin{array}{c}\text { Capsid associated; the nucleocapsids } \\
\text { stick in the virogenic stroma in } \\
\text { absence of } \mathrm{Vp} 80\end{array}$ & $\begin{array}{l}\text { Braunagel et al., 2003, } \\
\text { Marek et al., } 2011\end{array}$ \\
\hline P24 & ac129 & $\begin{array}{l}\text { Associated with BVs and ODVs, } \\
\text { capsid protein }\end{array}$ & $\begin{array}{l}\text { Braunagel et al., 2003, } \\
\quad \text { Wang et al., } 2010\end{array}$ \\
\hline ODV-EC27 & ac144 & $\begin{array}{l}\text { Associated with the nucleocapsids } \\
\text { with AC101, AC102 and PP78/83 }\end{array}$ & $\begin{array}{c}\text { Braunagel et al., 2001, Hepp et } \\
\text { al., } 2018\end{array}$ \\
\hline
\end{tabular}


Table 1 (continued)

\begin{tabular}{|c|c|c|c|}
\hline \multicolumn{4}{|c|}{ BV and ODV production } \\
\hline AC58/59 & $a c 58$ & $\begin{array}{c}\text { Non-essential BV and ODV } \\
\text { associated protein }\end{array}$ & $\begin{array}{l}\text { Braunagel et al., 2003, } \\
\text { Wang et al., } 2010\end{array}$ \\
\hline AC73 & $a c 73$ & $\begin{array}{l}\text { associated with AcMNPV BVs and } \\
\text { ODVs. Important for BV production } \\
\text { and infection in vivo }\end{array}$ & $\begin{array}{l}\text { Wang et al., 2010, } \\
\text { Shao et al., } 2019\end{array}$ \\
\hline GP41 & ac80 & $\begin{array}{c}\text { Core gene; tegument protein, } \\
\text { involved in nuclear egress of } \\
\text { nucleocapsids and ODV assembly }\end{array}$ & $\begin{array}{l}\text { Whitford et al., 1992a,b, } \\
\text { Olszewski et al., 1997b, } \\
\text { Li et al., 2018b }\end{array}$ \\
\hline AC81 & ac81 & $\begin{array}{c}\text { Core gene, required for nucleocapsid } \\
\text { envelopment and embedding of } \\
\text { ODVs }\end{array}$ & $\begin{array}{l}\text { Chen et al., 2007, } \\
\text { Dong et al., } 2016\end{array}$ \\
\hline $\begin{array}{l}\text { Sylfhydr ox } \\
\quad \text { / P33 }\end{array}$ & ac92 & $\begin{array}{l}\text { Core gene, associated with BVs and } \\
\text { ODVs, deletion results in } \\
\text { envelopment of single nucleocapsids }\end{array}$ & Wu et al., 2010, Nie et al., 2011 \\
\hline ODV-E25 & ac94 & $\begin{array}{l}\text { Core gene, associated with BVs and } \\
\text { ODVs, play a role in the shift from } \\
\text { BV to ODV production }\end{array}$ & $\begin{array}{l}\text { Russell et al., 1993, Braunagel } \\
\text { et al., 2003, Wang et al., 2010, } \\
\text { Chen et al., 2012, 2013, Luo et } \\
\text { al., 2013, Rohrmann et al., } 2013\end{array}$ \\
\hline AC109 & ac109 & $\begin{array}{l}\text { Core gene, BV and ODV associated, } \\
\text { various phenotypes observed in } \\
\text { virion production with mutants }\end{array}$ & $\begin{array}{c}\text { Braunagel et al., 2003, Fang et } \\
\text { al., 2009b, Wang et al., 2010, } \\
\text { Lin } \text { et al., 2009, Lehiy et al., } \\
2012\end{array}$ \\
\hline ODV-E18 & ac143 & Core gene, BV and ODV associated & $\begin{array}{l}\text { Braunagel et al., 2003, } \\
\quad \text { Wang et al., } 2010\end{array}$ \\
\hline AC66 & ac66 & $\begin{array}{c}\text { Core gene, facilitates nuclear egress } \\
\text { of nucleocapsids (with V-UBI and } \\
\text { AC141), and involved ODV and OB } \\
\text { production. }\end{array}$ & $\begin{array}{c}\text { Ke et al., 2008, } \\
\text { Biwas et al., } 2018\end{array}$ \\
\hline \multicolumn{4}{|c|}{ OB formation } \\
\hline AC5 & $a c 5$ & $\begin{array}{l}\text { Component of OB-matrix, unknown } \\
\text { function }\end{array}$ & Wang et al., 2018 \\
\hline POLH & $a c 8$ & $\begin{array}{l}\text { Matrix protein that crystallizes and } \\
\text { occludes the ODVs }\end{array}$ & Ji et al., 2010 \\
\hline PP34 & ac131 & Polyhedron envelope (calyx) protein & $\begin{array}{l}\text { Whitt and Manning, 1988, } \\
\text { van Lent et al., } 1990\end{array}$ \\
\hline \multicolumn{4}{|c|}{ Other } \\
\hline V-CATH & $a c 127$ & $\begin{array}{l}\text { BV associated, involved in } \\
\text { liquefaction of the insect host. }\end{array}$ & Lanier et al., 1996 \\
\hline AC132 & ac132 & $\begin{array}{l}\text { Associated with BVs and ODVs, } \\
\text { component of nucleocapsids and } \\
\text { ODV envelope, involved in nuclear } \\
\text { entry of nucleocapsids, might } \\
\text { function together with AC109 }\end{array}$ & $\begin{array}{c}\text { Braunagel et al., 2003, } \\
\text { Wang et al., 2010, Ono et al., } \\
\text { 2012, Fang et al., } 2016\end{array}$ \\
\hline
\end{tabular}


AC114 and GP37, which are important for oral infectivity, interact with components of the entry complex.

Two other non-PIF proteins that are important for the oral infectivity were also identified as PIF interaction partner. AC114 precipitated together with PIF8 and 9, and glycoprotein GP37 only with PIF9 (Fig. 1, 2). The acl14 gene is highly conserved among alphabaculoviruses and the encoded protein is associated with BVs and ODVs (Braunagel et al., 2003, Wang et al., 2010). This protein is important for the embedding of the ODVs into the polyhedrin matrix, as the OBs contained less ODVs after deletion of ac114, which reduced the OB infectivity significantly (Wei et al., 2012). GP37 was probably found because of the presence of polyhedrin after immunoprecipitation of PIF9, as GP37 associates with the polyhedrin matrix of the OBs. GP37 has affinity for chitin and enhances per os infectivity by facilitating the passage of ODVs through the peritrophic membrane (Vialard et al., 1990, Li et al., 2003, Liu et al., 2011). A homolog in betabaculoviruses was recently shown to have also fusogenic properties (Liu et al., 2019).

Other proteins that are known to be important for oral infectivity, AC18 (41 kDa), AC111 (8 $\mathrm{kDa}), \mathrm{AC} 124(29 \mathrm{kDa})$ and the enhancins AC145 (9 kDa) and AC150 (11 kDa) (Wang et al., 2007, Li et al., 2018, Lapointe et al., 2004, Zhang et al., 2005), were not identified as interaction partner of any of the immunoprecipitated PIFs. AC18 was detected after immunoprecipitation of PIF9, but was not significantly enriched compared to the wild type control (Supplementary data Fig. S2.2). The other proteins were not detected in any of the protein samples. These proteins were also not found when in a mass spectrometry analysis of the $480 \mathrm{kDa}$ band that is formed by the entry complex in blue native PAGE gels (Wang et al., 2019). However, this does not necessarily mean that they don't interact with PIFs as these proteins might be hard to detect by mass spectrometry due to their small sizes as explained before.

Other proteins involved in nucleocapsid assembly, virion production and OB formation.

Immunoprecipitation of PIF9 resulted in the co-precipitation of eighteen additional ODV proteins which are not described above. These proteins were categorized into four groups according to their reported function: nucleocapsid assembly (and actin rearrangement), virion production, OB formation and others (Table 1, Fig. 2). Four of these proteins, sulfhydryl oxidase (P33), P6.9, AC5 and P24, were also found after immunoprecipitation of PIF5 (Fig. 1, 2). The identification of PP34 and AC5, both OB components (Table 1), might be due to the presence of the polyhedrin matrix protein in the sample after immunoprecipitation of PIF9. Polyhedrin and AC5 were also found previously after immunoprecipitation of PIF1 (Peng et 
al., 2012). Sulfhydryl oxidase (P33) and P24 have been associated with the ODV entry complex before and are respectively involved in virion production and nucleocapsid assembly (Table 1) (Wang et al., 2019). AC73 was also found after immunoprecipitation of PIF9. This proteins only occurs in group I alphabaculoviruses and associates with the nucleocapsids of BVs and ODVs (Shao et al., 2019). The BV production was affected and in vivo infectivity was reduced after deletion of the ac73 gene (Shao et al., 2019). P6.9 is encoded by a core gene and is involved in capsid assembly, possibly because of its DNA binding properties (Wang et al., 2010, Lai et al., 2018). This protein is found associated with PIFs for the first time in this study after precipitation of PIF5 and PIF9. This is quite significant as this protein might be difficult to detect by mass spectrometry due to its small size and repetitive amino acid motif (GarciaMaruniak et al., 2004). This protein was detected before in the nucleocapsids of ODVs by mass spectrometry (Braunagel et al., 2003). These results indicate that PIF9 also interacts with capsid proteins. This is supported by the finding of all the components of another complex, that is formed at the base of the nucleocapsids by AC101, AC102, PP78/83 and ODV-EC27, and rearrange nuclear actin in the host cell (Hepp et al., 2018) (Fig. 2, Table 1).

PIF6 interacts with proteins that are essential for virion production.

Immunoprecipitation of PIF6 resulted in the precipitation of AC81, ODV-E18 and ODV-E25, in addition to the larger components of the entry complex (Fig. 1 and 2). AC81 was also found after immunoprecipitation of PIF5 (Fig. 1 and 2). These proteins seemed to interact specifically with PIF6 as they were not found after immunoprecipitation of PIF8 or 9 and had also not been found after immunoprecipitation of PIF1 before (Peng et al., 2012). These proteins have also been found in the $480 \mathrm{kDa}$ band in blue native-PAGE gels, representing the entry complex (Wang et al., 2019), and are all encoded by core genes and essential for virion production (Table 1).

\section{Conclusion}

This study shows that the PIFs not only interact with each other in the ODV entry complex, but also with various non-PIF proteins that are present in the ODV envelope, which are important for the formation of virions and OBs. This was especially the case for PIF9, that was found to interact with various components of the nucleocapsids. It was also shown that PIF5 interacts with two components of the entry complex, PIF0 and 1, and not with any other component of the ODV entry complex. This suggests that PIF0 and 1 not only occur in the entry complex, but also outside the complex in interaction with PIF5. The biological relevance of this interaction 
with PIF5 for the infectivity of ODVs remains to be established. These findings indicate that, despite formation of the entry complex, the PIFs also function in concert with other ODV proteins. These interactions might be important for the complete infectious potential of ODVs to larval midgut cells.

\section{Materials and methods}

Insect cells and viruses. TnH5 (from Trichoplusia ni) and Sf21 cells (from Spodoptera frugiperda) were cultured in Grace's medium (Thermo Fisher), supplemented with $10 \%$ fetal bovine serum (FBS) and $50 \mu \mathrm{g} / \mathrm{ml}$ gentamycin (Gibco, Life technologies) at $27^{\circ} \mathrm{C}$. Bacmid derived AcMNPV E2-strain (bMON14272) with a restored polh gene was used as wild type in this study. Viruses that encode HA-tagged PIF proteins have the same genetic origin. The HAtagged PIF5 and 9 viruses have been described before in Peng et al., 2012 and Chapter 4. Bacmids for the HA-tagged PIF6 and 8 viruses were kindly provided by dr. D.A. Theilmann of the Summerland Research and Development Centre for Agriculture and Agri-Food, Canada.

Production of the mutant viruses. BV-stocks of the HA-tagged viruses were produced by transfection of Sf21 cells with the respective bacmids using Expres2TR transfection reagent (Expres2ion Biotechnologies), followed by an additional infection round in Sf21 cells as described previously (Chapter 3, 4). OBs were produced by infection of TnH5 cells with BVs at an MOI of 2 and isolated as described before (Chapter 4). In brief, the infected cells were harvested at six days post infection by centrifugation at $4000 \mathrm{x}$ g for $30 \mathrm{~min}$ and lysed by incubation in $0.1 \%$ SDS for $2 \mathrm{hrs}$ at $37^{\circ} \mathrm{C}$ with gentle agitation and subsequent sonication for 1 min with an intensity of 8 Watt on ice. Complete lysis was verified by light microscopy and the cells were sonicated once more when needed. When all the cells were lysed, the OBs were pelleted by centrifugation at $4000 \mathrm{x}$ g for $30 \mathrm{~min}$. and washed three times with sterile double distilled water.

Immunoprecipitation with anti-HA magnetic beads. The ODVs from $\sim 5 \times 10^{8}$ OBs of the HAtagged mutants were isolated and fractionated in triplicate in a slightly modified way from what was described in Chapter 4. The ODVs were fractionated by a $2 \mathrm{hr}$ incubation in protein extraction buffer (25mM Tris, $150 \mathrm{mM} \mathrm{NaCl}, 0.5 \%$ Triton X-500 pH 7.2), followed by two short sonication bursts. The envelope fractions were incubated overnight with $20 \mu \mathrm{l}$ anti-HA magnetic beads (Invitrogen) in $200 \mu 1$ protein extraction buffer at $4^{\circ}$ with gentle rotation. Wild type ODVs (without HA-tag) were treated in parallel and were used as a negative control. The 
beads were washed for three times with extraction buffer and the captured proteins were eluted from the beads by incubation in Laemmli buffer at $95^{\circ} \mathrm{C}$ for $10 \mathrm{~min}$.

SDS-PAGE and western blot analysis. The proteins were loaded on two different Any-Kd TGX precasted protein gels (Biorad). The first gel was used to verify the precipitation of the target protein and the known interaction partners by western blot analysis, with either anti-HA antibodies (Roche 3F10, 1:2000, rat-derived) or antiserum against PIF0, produced in rabbit, 1:1000; PIF1, produced in rats, 1:2000 (Peng et al., 2010) and PIF8, produced in rabbit, 1:2000 (Russell and Rohrmann, 1997). Goat anti-rabbit (1:2000 dilution; Dako) and goat anti-rat (1:2000 dilution; Sigma A8438), conjugated to alkaline phosphatase, were used as secondary antibodies for detection of the proteins of interest by conversion of NBT-BCIP substrate in a blue-purple coloured precipitant in AP-buffer (0.1 M Tris- $\mathrm{HCl}, 0.1 \mathrm{M} \mathrm{NaCl}, 5 \mathrm{mM} \mathrm{MgCl}, \mathrm{pH}$ 10.5). The second gel was run until the loading dye migrated $2.5 \mathrm{~cm}$ into the gel and was stained with oriole (Biorad). The proteins in this gel were analysed by mass spectrometry after in gel digestion with trypsin as described in the next section.

In gel digestion and MS/MS sample preparation. The proteins were reduced and alkylated prior to in-gel digestion with trypsin. The proteins were reduced by a $1 \mathrm{hr}$ incubation in $50 \mathrm{mM}$ dithiothreitol (DTT), dissolved in $50 \mathrm{mM}$ ammonium bicarbonate (ABC; Sigma) $\mathrm{pH}$ 8.0, and alkylated by incubation in $20 \mathrm{mM}$ acrylamide (Sigma), dissolved in $0.1 \mathrm{M}$ Tris; $\mathrm{pH}$ 8.0, for 30 min in the dark. Both treatments were performed at room temperature. The lanes that were loaded with proteins were cut into five slices under UV-light and subsequently into small blocks of approximately $1 \mathrm{~cm}^{2}$. These blocks were washed with $50 \mathrm{mM} \mathrm{ABC}$ buffer and stored overnight at $-20^{\circ} \mathrm{C}$ to increase the trypsin accessible area. The proteins were digested by overnight incubation with $5 \mathrm{ng} / \mu \mathrm{l}$ bovine sequencing grade trypsin (Roche) in $50 \mathrm{mM} \mathrm{ABC}$ at room temperature. The $\mathrm{pH}$ was set to 3 by the addition of $10 \%$ trifluoro acetic acid and the peptides were purified over C18 $\mu$ Columns. The peptides were analysed by LC-MS/MS using the LTQ-Orbitrap as described previously in Kariithi et al., 2010.

The raw data were analysed by MaxQuant software (version 1.6.3.4) for (label free) quantification and identification of the peptides by searching against the AcMNPV Uniprot 2017 database (Cox et al., 2008, 2011). A standard contamination database was also used. Modifications that were included for protein quantification were: oxidation $(\mathrm{M})$, acetylation (Protein N-term) and deamination (NQ). The datasets were filtered with Perseus to select only 
the proteins that were identified with minimally two peptides (with a minimal peptide length of seven amino acids) of which at least one is unique and one is unmodified (Hubner et al., 2010).

\section{Acknowledgements}

We like to thank Dr Sjef W. Boeren of the Laboratory of Biochemistry for his support and mass spectrometry analysis. This work was supported by the Strategic Fund of the graduate school Production and Ecology \& Resource Conservation, The Netherlands. 


\section{Supplementary data}

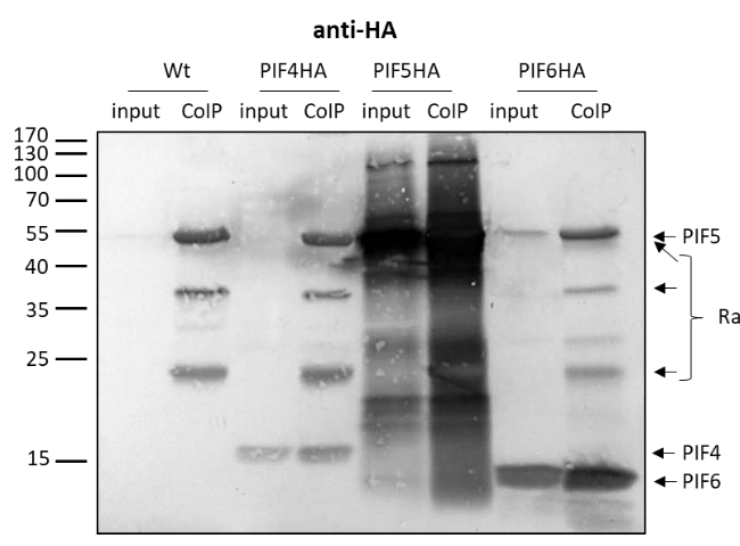

(a)

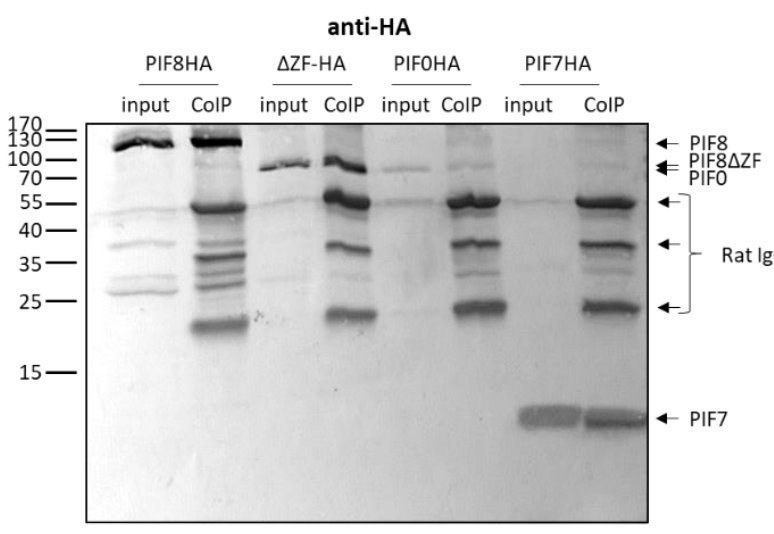

(c)

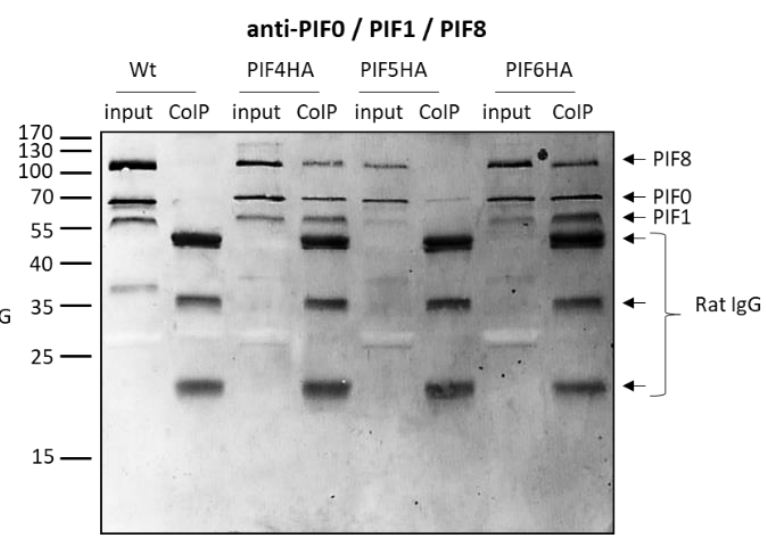

(b)

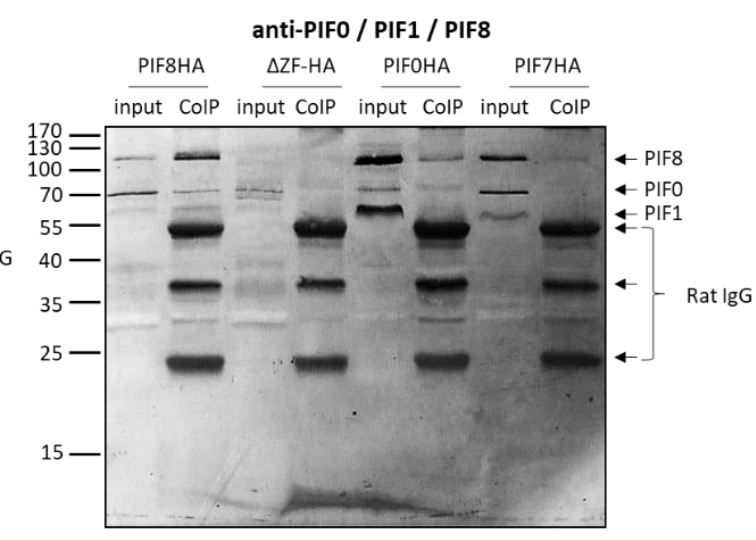

(d)

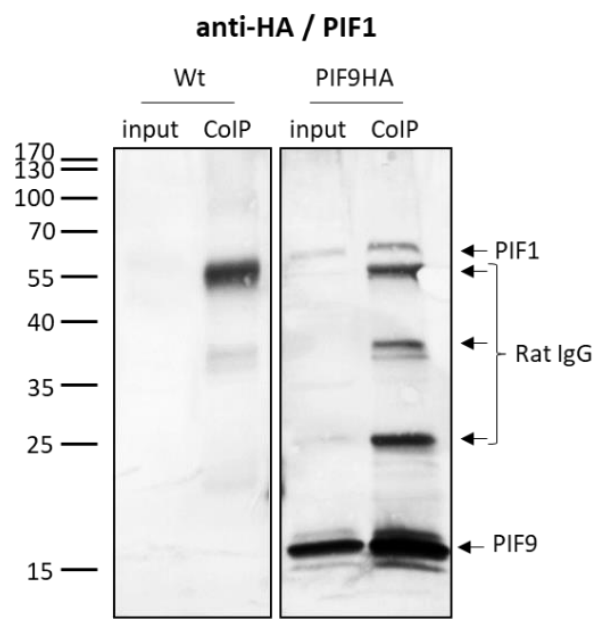

(e)

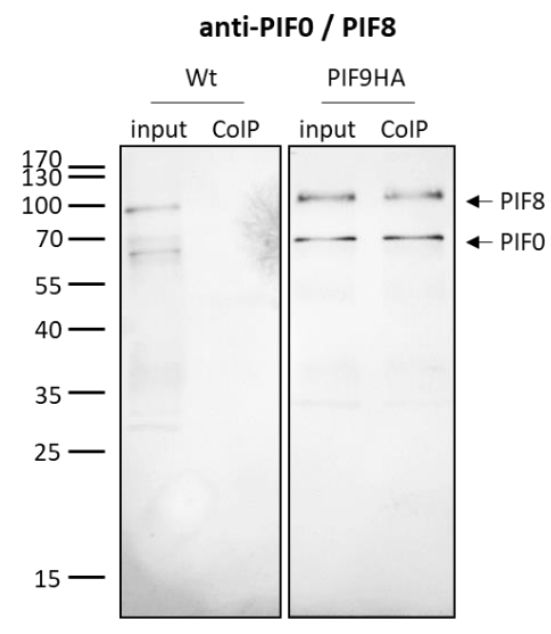

(f)

Figure S1: Validation of precipitation of HA-tagged PIF5, 6, 8 and 9 with the anti-HA magnetic beads. Immunoprecipitated proteins were analysed by western blot with anti-HA antibodies in (a), (c) and (e); and antisera against PIF0, 1 or 8 in (b), (d) and (f). The results after immunoprecipitation of HA-tagged PIF4, 5 and 6 are shown in (a) and (b); PIF8, PIF8 $\mathrm{ZF}$, PIF0 and PIF7 in (c) and (d); and PIF9 in (e) and (f). Immunoprecipitation of PIF0 and PIF7 were excluded from analysis by mass spectrometry as PIF0 failed to precipitate efficiently and PIF4 and 7 appeared not detectable by this method. 

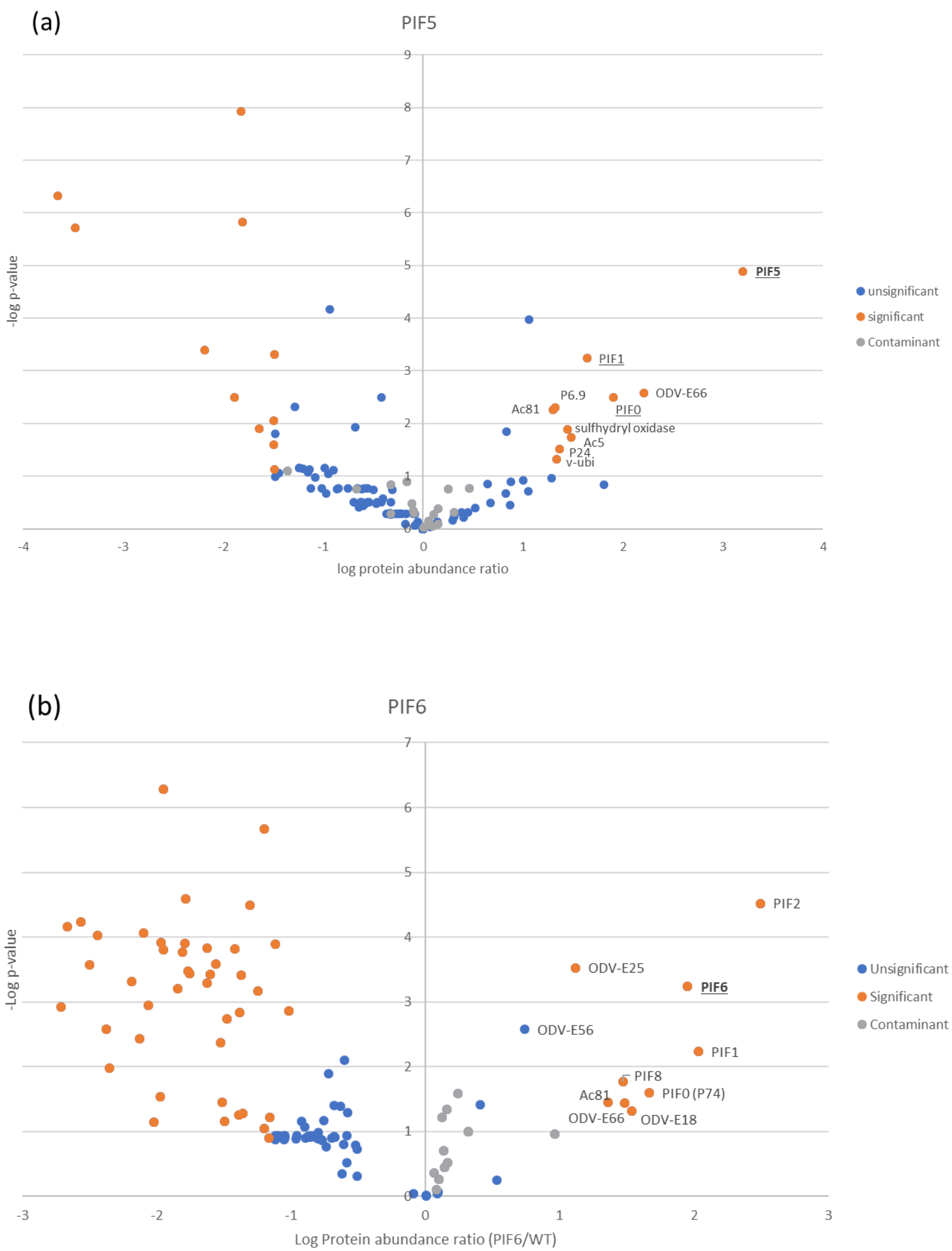

Figure S2.1: Vulcano plots of the identified proteins after immunoprecipitation of PIF5 and PIF6. The Xaxis shows the $\log (10)$ protein abundance ratio, indicating the enrichment factor over wild type (wt). The Y axis shows the - $\log$ p-value of student t-tests of difference in label free quantification (LFQ) values between wild type and after immunoprecipitation of one of the PIFs, indicating the statistical significance of the hits. The hits were considered as significant with $\log$ protein abundance ratio $>1.2$ over wt ( $15 \mathrm{x}$ enriched) and $-\log \mathrm{p}$-values $>1.3$ $(\mathrm{p}<0.05)$. Significant hits are depicted in orange, non-significant hits in blue and contaminants, as human keratins or immunoglobulins, in grey. 


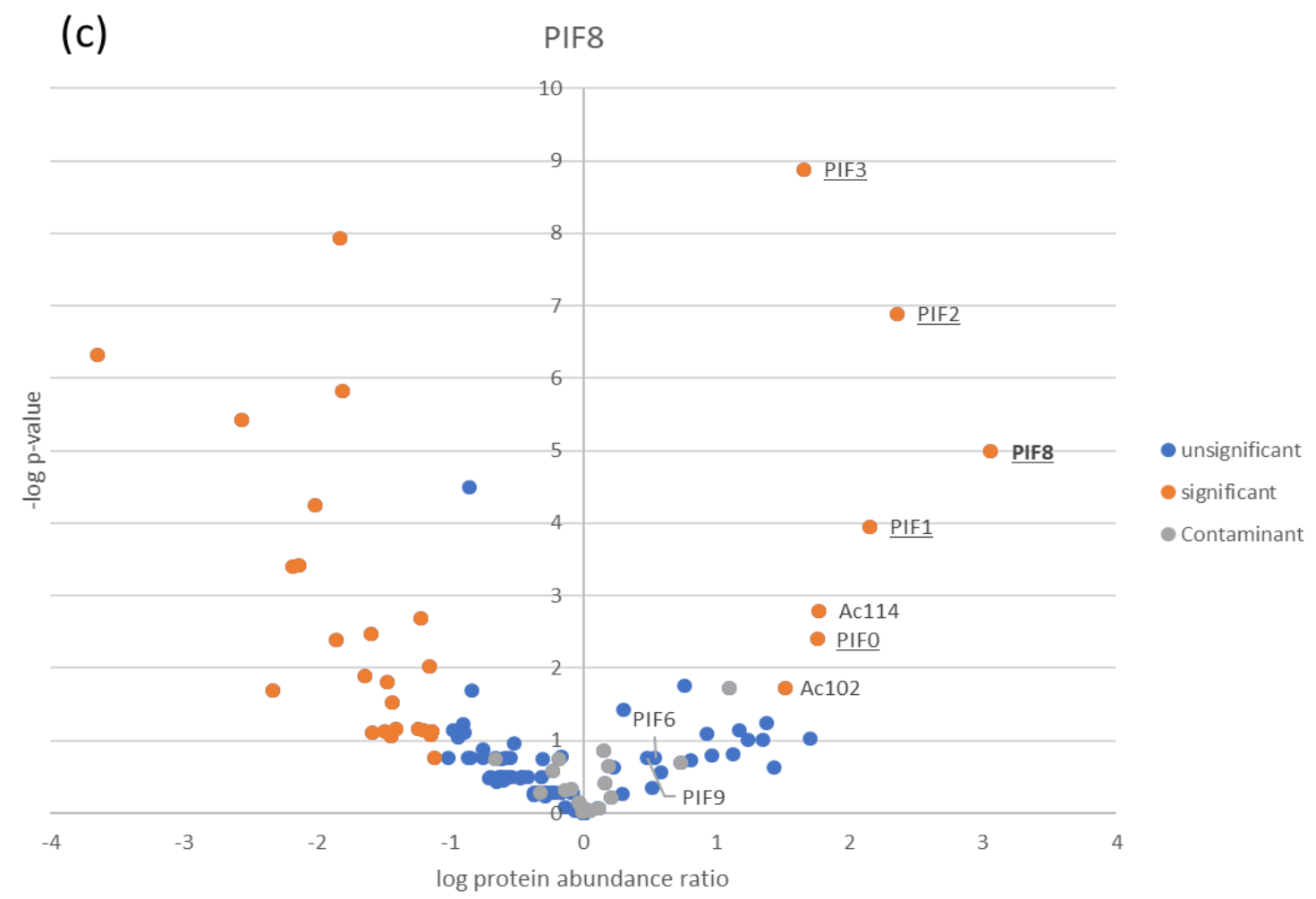

(d)

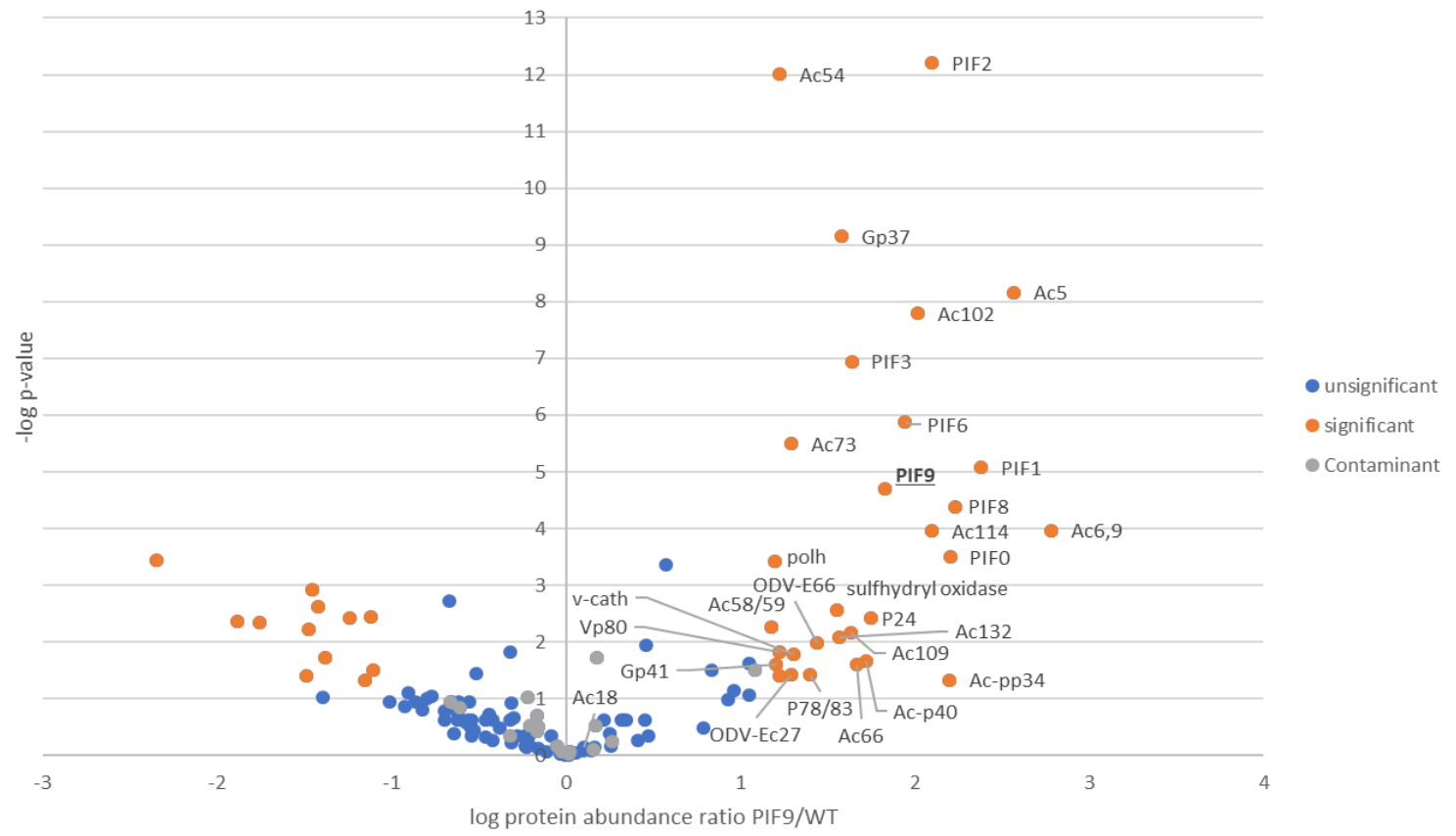

Figure S2.2: Vulcano plots of the identified proteins after immunoprecipitation of PIF8 and PIF9. The xaxis shows the $\log (10)$ protein abundance ratio, indicating the enrichment factor over wild type (wt). The $\mathrm{Y}$ axis shows the - $\log \mathrm{p}$-value of student t-tests of difference in label free quantification (LFQ) values between wild type and after immunoprecipitation of one of the PIFs, indicating the statistical significance of the hits. The hits were considered as significant with log protein abundance ratio $>1.2$ over wt (15x enriched) and -log p-values $>1.3$ $(\mathrm{p}<0.05)$. Significant hits are depicted in orange, non-significant hits in blue and contaminants, as human keratins or immunoglobulins, in grey. 


\section{Chapter 8}

General discussion 


\section{Introduction}

The baculovirus occlusion derived viruses (ODVs) infect larval midgut epithelial cells under highly alkaline conditions. For this, the viral envelope contains ten different proteins, called per os infectivity factors (PIFs). Nine of these proteins form the ODV entry complex (Peng et al., 2010, 2012, Chapter 2, 3 and 4). Per definition, the PIFs are only crucial for the infection of midgut epithelium cells by ODVs and are not involved in cell-to-cell spread of the infection by budded viruses (BVs). Viruses with pif gene deletions replicate in cultured insect cells without any noticeable effect on the production of BVs, ODVs and occlusion bodies (OBs). PIF proteins are highly conserved among baculoviruses and some of them also in related invertebrate infecting viruses, suggesting that this mechanism of virus entry into cells underwent millions of years of evolution (Thézé et al., 2011). However, the molecular mechanism of PIF-mediated entry is still elusive to date. In this thesis, the ODV entry complex was biochemically and functionally characterised.

The complexity of the baculovirus entry process in the midgut.

The finding of ten different genes in the baculovirus genome that encode for PIF proteins suggests that the entry of ODVs into larval midgut epithelial cells is a joint effort and that the functioning of a single PIF strongly depends on the interaction with other PIFs. Indeed, nine out of the ten PIFs are crucial for the formation of the ODV entry complex. PIF1 to 4 form the stable core of the entry complex and the five remaining PIFs, i.e. PIF0 and PIF6 to 9, associate more loosely to the complex (Peng et al., 2010, 2012, Chapter 2, 3 and 4). However, most studies to unravel the biological function of the PIFs in midgut entry were conducted without the knowledge that these proteins form such a complex. Chapter 2 of this thesis therefore reviewed the existing literature on PIFs, discussing these proteins in relation to each other, rather than as separate proteins with a similar function in the baculovirus infection cycle. It was concluded that the existence of the entry complex complicates the interpretation of earlier studies with respect to the biological function of each individual PIF. Therefore, it can be questioned whether the observed phenotype with a pif deletion mutant is truly the effect of the absence of that specific PIF or an indirect effect due to disturbed protein interactions. The last option is retrospectively more likely.

This idea is supported by the results obtained with PIF1 and PIF2 C-terminal truncation mutant viruses (Chapter 6). PIF1 and 2 had been reported as being important for the binding of ODVs to the midgut epithelium (Ohkawa et al., 2005) and for formation of the entry complex (Peng 
et al., 2010). As binding to the midgut epithelium is a host range determinant (Haas-Stapleton et al., 2005) and most baculoviruses have a narrow host range, we hypothesized that the highly conserved N-terminal part of PIF1 and 2 is important for complex formation and that the less conserved C-terminal parts of these PIFs interact with the host. By generating PIF1 and PIF2 C-terminal truncation mutant viruses, it was attempted to distinguish these two functions by the identification of a mutant virus that is able to form a complex while lacking oral infectivity. However, small truncations from the C-terminus of one of those PIFs already affected the formation of the entry complex as at least two of the loosely associated components, PIF0 and 8 , failed to bind with the stable core of the entry complex. Also, the oral infectivity was severely affected in these mutant viruses (Chapter 6). This indicates that PIF1 and 2 mediate ODV infectivity in close association with the other PIFs and in the case that these PIFs are directly involved in host interaction, they are probably only exposed in the right conformation after formation of the complex. These results also demonstrate that it is impossible to study the functionality of individual PIFs by mutagenesis without affecting the formation of the entry complex and thus also the functioning of the remaining PIFs. In addition, we now know that the entry complex also fails to assemble in absence of one of the loosely associated components (Peng et al., 2010, Javed et al., 2017, Chapter 3 and 6). Therefore we conclude that these looser PIFs also depend on each other's presence to bind to the complex and probably also for their proper functioning.

\section{Formation of the entry complex is essential to resist the proteolytic activity of host proteases}

Although the molecular mechanism of the entry complex and the biological role of individual PIFs are difficult to dissect due to the above discussed reason, the results presented in Chapter 3 provide a first clue to why this complex is formed. ODVs derived from larvae are, in contrast to ODVs from cultured insect cells (Peng et al., 2011), subjected to the proteolytic activity of alkaline proteases from the host. These host proteases are co-occluded in the OBs (Payne and Kalmakoff, 1977, Wood et al., 1980) and are activated when the OBs are dissolved under alkaline conditions (Peng et al., 2011). In Chapter 3 it was shown that when PIFs of wild type ODVs were analysed under denaturing conditions after dissolution of the OBs in alkaline buffer, PIF monomers were found in both cell culture- and larva-derived virus suspensions. However, when one of the PIFs was absent in any of the pif deletion mutants tested and as a consequence the formation of the entry complex was abolished, the monomeric form of the remaining PIFs were only found in cell culture-derived ODVs and not in larva-derived ODVs. These monomeric PIFs were also observed again when the larval OBs were heat-treated to 
inactivate the proteases prior to the dissolution in alkaline buffer. These experiments were carried out with a number of pif deletion mutant viruses and showed that when PIFs occur in monomeric form, these are prone to proteolytic degradation by host proteases. Furthermore, the PIFs in the stable core (PIF1 to 4) were relatively resistant against these proteases compared to the PIFs in monomeric form. The tight interaction of the PIFs in the complex may be needed to cope with the harsh environment of the larval midgut, in which the virions are heavily exposed to digestive proteases. These results emphasize once again the strong dependency between the PIFs.

How these proteases end up in the OBs and what their functional significance is, is currently unclear. Is has been proposed that these proteases aid in the release of the ODVs by degrading the matrix protein polyhedrin (Wood et al., 1980). The co-occluded proteases have also been shown to cleave PIF0 into two fragments of 35 and $40 \mathrm{kDa}$, which both remain associated with the complex (Peng et al., 2011). The entry complex also prevents further cleavage of PIF0 as this protein was degraded when complex formation was abolished in mutants with other pif gene deletions (Chapter 3). The importance of this PIF0 cleavage event for midgut infection is presently enigmatic. PIF0 is cleaved for a second time in the gut by host trypsins, which cleave off a $20 \mathrm{kDa}$ piece from the $35 \mathrm{kDa}$ N-terminal fragment (Slack et al., 2005, 2008). This cleavage event has been shown to be important for the infectivity of ODVs (Slack et al., 2008). These two cleavage events possibly activate PIF0 and maybe indirectly also other PIFs by inducing conformational changes in the entry complex, which are important for ODV entry into epithelial midgut cells.

\section{The importance of entry complex formation for ODV binding and fusion}

With respect to binding and fusion, different phenotypes have been observed in R18 dequenching assays with various pif deletion mutant viruses (Ohkawa et al., 2005). We now know that these mutants are not able to form the complex (Peng et al., 2010). Absence of either PIF0, 1 or 2 affected ODV binding and not fusion (Haas-Stapleton et al., 2004, Ohkawa et al., 2005), while in absence of PIF3 both binding and fusion were unaffected (Ohkawa et al., 2005). The pif 3 deletion mutant virus nevertheless failed to establish a midgut infection. This suggests that formation of the entry complex is not important for the binding and fusion capacities of ODVs, while this was initially expected to be the main function of the complex. To determine the biological importance of the entry complex, apart from protection of the individual PIFs against proteases as demonstrated in Chapter 3, the involvement of PIF3 in midgut infection 
was further studied by monitoring fluorescently labelled ODVs of a pif 3 deletion mutant in their interaction with the brush borders of isolated epithelial midgut cells of S. exigua larvae (Chapter 5). The pif3 mutant ODVs bound to the brush borders, but the nucleocapsids failed to enter the cell, in contrast to capsids of the wild type (labelled) virus. It has been proposed by others that PIF3 is important for translocation of the nucleocapsids to the microvilli (Ohkawa et al., 2005). This possibility was not excluded by our studies as membrane fusion cannot be observed in the assay with ODVs that have fluorescently labelled capsids. We could only see that the capsids did not move from the place of initial interaction with the brush border. An alternative explanation is that only the outer leaflets of the cellular and viral lipid bilayers fused (a hemifusion state), which also has been shown to result in R18 dequenching with influenza and vaccinia mutant viruses (Kemble et al., 1994, Laliberte et al., 2011). The formation of the entry complex would according to this theory be important to continue to a full membrane fusion stage. Furthermore, ODVs of another complex-deficient mutant virus, that lacks PIF9, showed the same phenotype. However, the fusogenic properties of pif9 mutant ODVs need to be determined by R18 dequenching assays in future research. These experiments nevertheless show that when formation of the entry complex is abolished, the infection process is halted at an early stage at the brush border of the midgut cell as the nucleocapsids then fail to enter the cell.

Is the entry complex supported by other envelope proteins to achieve optimal ODV infectivity?

Various studies showed that the individual PIFs are important for the primary infection of the larval midgut. This thesis examined the relation between complex formation and oral infectivity, in which it was shown that absence or mutation of a single PIF protein abolished complex formation and infectivity of the ODVs (Chapter 4, 5, 6). However, mutant viruses with a C-terminal truncated version of PIF1 or 2, which are deficient in complex formation as well, were incidentally able to infect the larvae (Chapter 6). It has also been described for various other pif mutant viruses that they caused a low incidence of infection (Ohkawa et al., 2005, Fang et al., 2009, Dong et al., 2014, Zhu et al., 2013 and Wang et al., 2019), while these mutants are now known not to be able to form the entry complex (Peng et al., 2010, 2012, Javed et al., 2017, Chapter 3, 4, 6). Furthermore, the infectivity of a complex-deficient pif8 mutant, that lacks the ZF-domain, can be partially rescued by pre-treatment of the larvae with calcofluor white, an agent that damages the peritrophic membrane (Zhu et al., 2013). This suggests that PIF8 targets the peritrophic membrane and that the remaining PIFs exert some (remnant) biological activity, despite the inability to form the entry complex. 
An alternative explanation for these low incidence infections is that other ODV envelope proteins support the ODV entry complex and to a certain extent might compensate for a lacking PIF and the affected complex formation in mutant viruses. PIF5 and ODV-E66 for example are also important for ODV oral infectivity (Harrison et al., 2010, Sparks et al., 2011a, b), but are not associated with the entry complex (Peng et al., 2012, Wang et al., 2019). A significant difference between these proteins and the PIFs in the entry complex is that absence of either PIF5 or ODV-E66 results in a quantifiable increase in the lethal dose (LD50: the required dose to reach 50\% mortality) of the mutant ODVs, while absence of one of the constituents of the entry complex has such an impact on the oral infectivity that only a single larva might incidentally be infected at a dose that normally would kill all larvae. In Chapter 7, the first data have been obtained that PIF5 and ODV-E66 are interaction partners in the ODV envelope, as immunoprecipitation of PIF5 resulted in the co-precipitation of ODV-E66. This PIF5 pull-down also resulted in the precipitation of PIF0 and 1. That only these two PIFs were found to interact with PIF5 and not any other component of the ODV entry complex, suggests that these PIFs also occur outside the entry complex. These three interaction partners of PIF5 are needed for ODV binding (Haas-Stapleton et al., 2004, Ohkawa et al., 2005, Sparks et al., 2011b). However, the binding and fusion capacities of the ODVs of a pif5 deletion mutant were unaffected (Sparks et al., 2011a), suggesting that the interactions between PIF5 and ODV-E66, PIF0 or PIF1 are not required for ODV binding. The biological importance of these interactions for the infectivity of ODVs therefore remains unclear.

In addition, there are ODV envelope proteins that are only involved in oral infectivity in specific virus-host combinations, adding an extra layer of complexity. AC111 was found important for the infection of Trichoplusia ni larvae, but dispensable for the infection of Spodoptera exigua (Li et al., 2018). HA100 in the Helicoverpa armigera NPV is also involved in infectivity and only occurs in group II alphabaculoviruses (Luo et al., 2011). Furthermore, the viral glycoprotein GP37 that is known to be important for passage through the peritrophic membrane, might also be involved in membrane fusion as it was recently shown to have fusogenic properties (Liu et al., 2019). The ODV entry complex remains the main actor of ODV oral infectivity in larvae, but to thoroughly understand the infection process of the larval midgut epithelial cells, it is important to also take the other players and the putatively dual functions of the PIF proteins into account. 


\section{Comparison of the ODV entry complex with entry complexes of other viruses}

The formation of a protein complex that is crucial for virus entry has also been shown for members of the Herpesviridae and Poxviridae families. The entry complex in herpesviruses consists of three to six different proteins, depending on the virus species. A heterodimer of glycoproteins $\mathrm{gH}$ and $\mathrm{gL}$ forms the basis of the entry complex and activates a trimer of $\mathrm{gB}$ fusion proteins upon receptor binding. The species specific components of the complex establish tissue tropism by the binding of cell-specific host receptors (reviewed by Sathiyamoorthy et al., 2017). Herpes simplex virus for example adds glycoprotein gD to the complex for receptor binding (Gianni et al., 2009, Atanasiu et al., 2010) and the cytomegalovirus (CMV) adds either $\mathrm{gO}$ for the infection of fibroblasts (Vanarsdall et al., 2011, Ciferri et al., 2015, Kabanova et al., 2016) or a UL128-131 tetramer to infect epithelial, endothelial and myeloid cells (Ryckman et al., 2006). The finding of a variable complex composition and tropism among the herpes virus species is quite different from what is known from the baculovirus entry complex. The components of the ODV entry complex are highly conserved among baculovirus species and mediate entry into a single cell type, the columnar epithelial cells in the larval midgut. An intriguing similarity, however, is that both complexes contain a component, the $\mathrm{gHgL}$ dimer in herpesviruses and PIF0 in baculoviruses, that was shown to be able to function in trans during cell entry in experimental setups (Atanasiu et al., 2010, Yao et al., 2004, Slack et al., 2010).

The entry complex in the poxviruses shows more resemblance with the baculovirus ODV entry complex, especially when looking at the features of its components. This complex is formed by small proteins, nine to eleven in total that range from 4.2 to $44 \mathrm{kDa}$ in size, and that have $\mathrm{N}$ - or C-terminal transmembrane domains and conserved cysteine residues (Reviewed in Moss et al., 2016). Like most PIFs among baculoviruses, these proteins are highly conserved among the poxviruses. However, the complex in poxviruses mediates only membrane fusion and is not involved in the attachment of virus particles to the host cell. This provides the opportunity to study the fusogenic properties of the complex and its individual components without the risk of affecting virus attachment. This in contrary to the baculovirus entry complex where some essential components mediate ODV binding (Haas-Stapleton et al., 2004, Ohkawa et al., 2005, Mu et al., 2014). Although the absence of a single component also abolished complex formation in poxviruses, just as observed with the entry complex in baculoviruses (Chapters 4 and 6), two stages in the fusion process were distinguished (Laliberte et al., 2011). Eight out of eleven components were crucial for fusion as absence of one of them completely blocked membrane 
fusion. In contrast to the poxviruses, no baculovirus mutants have been identified in which fusion was totally blocked, not even for mutant ODVs with diminished binding capacities (Haas-Stapleton et al., 2004, Ohkawa et al., 2005). However, in absence of one of the other three components of the poxvirus entry fusion complex, lipid mixing was still observed, although capsid entry and content mixing failed. A two-step fusion model with a hemifusion intermediate was proposed for poxvirus entry, in which the outer leaflets of the lipid bilayers are apparently still able to mix in absence of the entry complex, but the capsids cannot enter due to the failure to form a fusion pore. This is also suggested for ODV entry in midgut cells, since similar results were obtained with the entry complex deficient mutant virus that lacks PIF3 (Ohkawa et al., 2005, Chapter 5). Hemifusion with poxviruses was also observed when the host cell membrane was depleted from cholesterol and upon treatment with inhibitors of tyrosine kinases and actin dynamics (Laliberte et al., 2011). These aspects of virus entry are hard to study with baculovirus ODVs, because of the lack of an in vitro cell culture system that models the larval midgut under alkaline conditions.

A model for the infection of midgut cells by $O D V s$.

This $\mathrm{PhD}$ thesis showed that the molecular action of the entry complex during ODV entry into midgut cells is hard to dissect because of the intimate collaboration between the PIFs, in which a small mutation of one of the pif genes possibly results in secondary effects due to abolished complex formation (Chapter $\mathbf{4}$ and 6). Furthermore, the infection process in the larval midgut might even be more complicated as additional factors are required for a fully infectious virus (Chapter 7). Nevertheless, a model of midgut

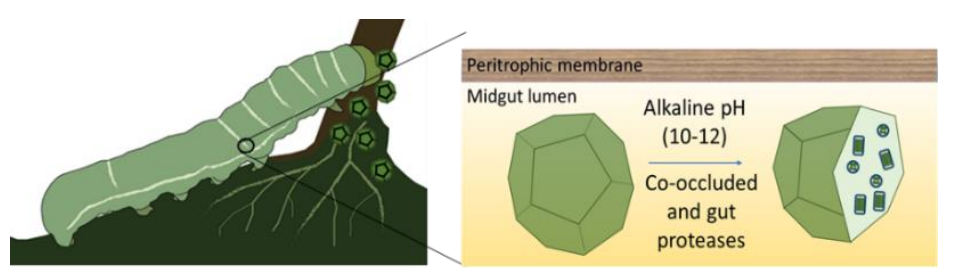
ODV envelope

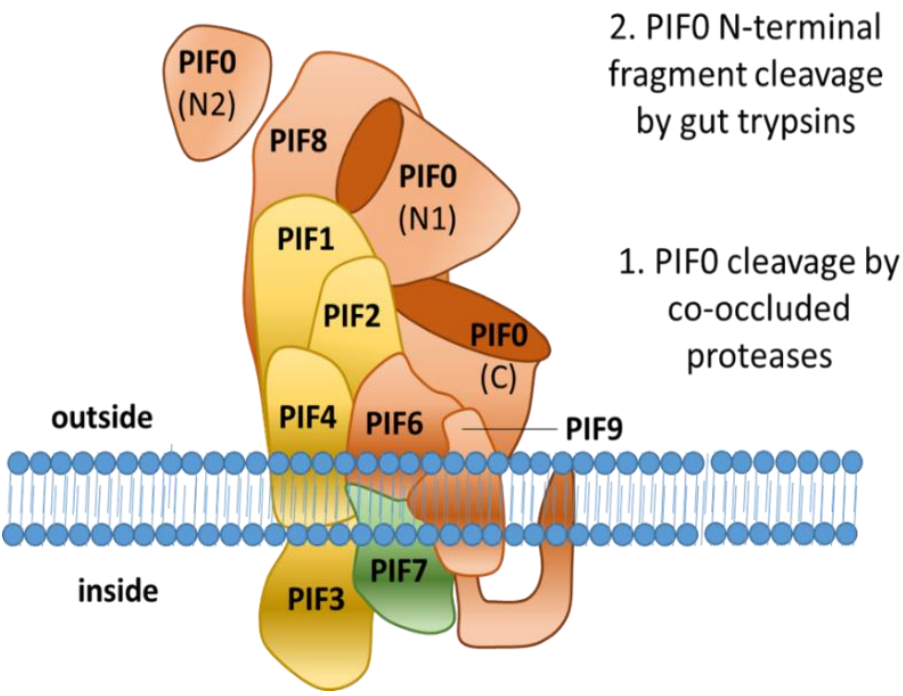

Figure 1: Activation of PIF0 in the midgut by proteolytic cleavage. The ODV entry complex ensures proper cleavage of PIF0 by co-occluded host proteases into two fragments. Both $\mathrm{N}$ - and $\mathrm{C}$ terminal fragments remain associated with the complex (Peng et al., 2011). A $20 \mathrm{kDa}$ piece is cleaved off from the $\mathrm{N}$-terminal fragment by trypsins in the gut, generating N1 and N2 (Slack et al., 2008). Whether these fragments remain associated to the complex is unknown. 
infection by ODVs will be presented here based on a combination of the experimental results in this thesis and literature data.

In this model, the ODVs are released into the midgut lumen after ingestion of OBs by insect larvae, where these are exposed to digestive enzymes in a highly alkaline environment. Together with the co-occluded proteases in the OBs, the gut proteases facilitate the release of the ODVs by degradation of the polyhedron-shaped protein matrix, in which they are embedded (Payne and Kalmakoff, 1977, Wood et al., 1980). The proteins in the ODV envelope are also subjected to the proteolytic activity by these proteases (Wood et al., 1980), in which the ODV entry complex allows the cleavage of PIF0 into two fragments, but prevents further degradation of PIF0 and degradation of other PIFs (Peng et al., 2011, Chapter 3). The two fragments of PIF0 remain associated to the complex (Peng et al., 2011), and the N-terminal fragment is further processed by trypsins by cleaving off a $20 \mathrm{kDa}$ piece from the $\mathrm{N}$-terminal fragment into N1 and N2 (Fig. 1) (Slack et al., 2005, 2008). The trypsin-mediated cleavage is important for ODV infectivity (Slack et al., 2008). A two-step cleavage model has been proposed in which PIF0, and may be the associated PIFs as well, are activated for binding and fusion with the epithelial cell brush border (Peng et al., 2011). However, after release of the ODVs into the gut lumen, the virions first need to pass the peritrophic membrane before reaching the epithelial cells. The peritrophic membrane is possibly targeted by PIF8 (Fig. 2), as the infectivity of PIF8 mutant ODVs was partially rescued when damaging this membrane with calcofluor white (Zhu et al., 2013). PIF5 interaction partner ODV-E66 has recently also been shown to be involved in this as well (Hou et al., 2019).
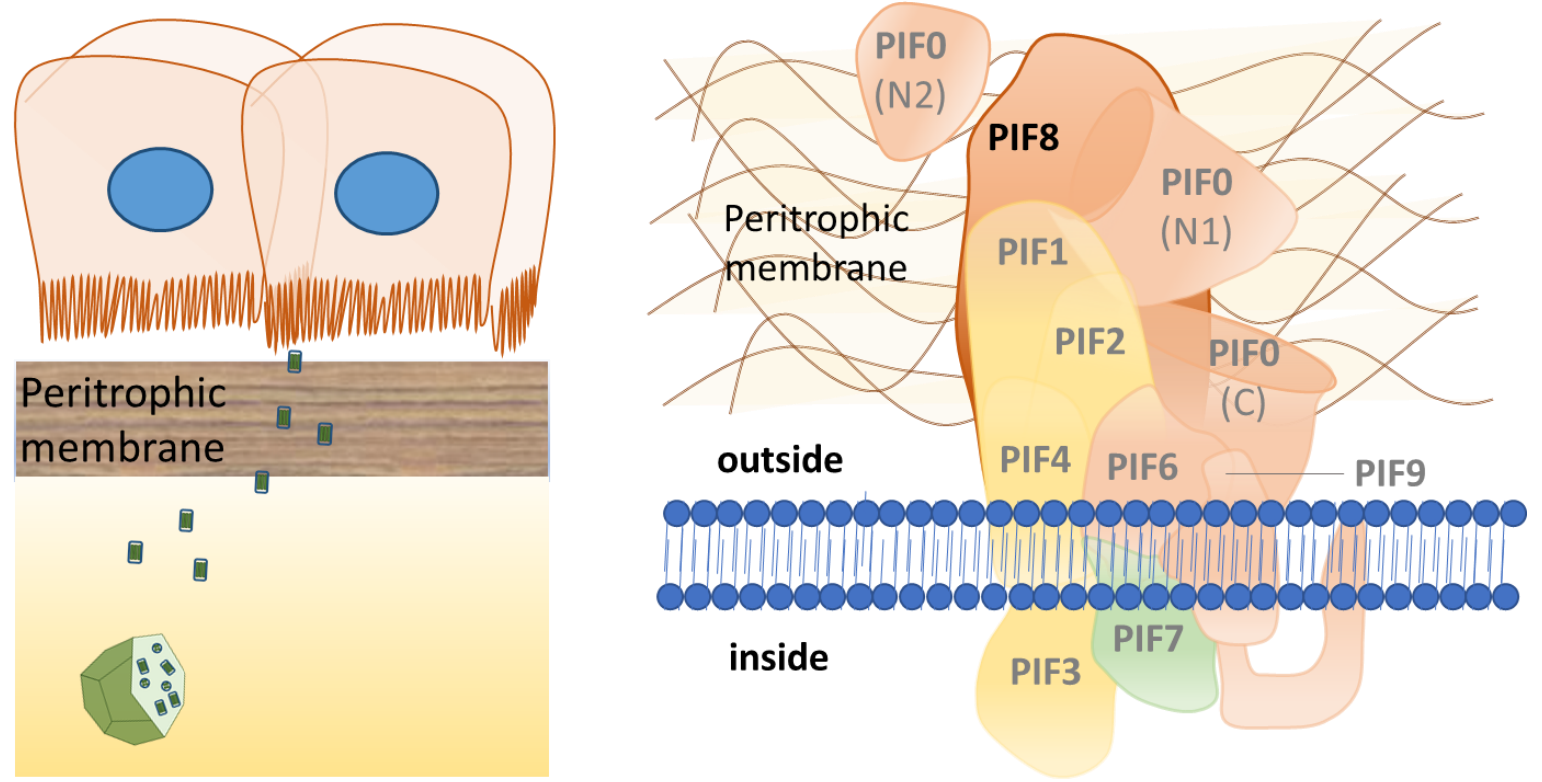

Figure 2: PIF8 is involved in passage of the peritrophic membrane 
Once the ODVs have passed the peritrophic membrane, the proteolytically activated PIF0 mediates ODV binding together with PIF1 and 2 via the interaction with an unknown receptor (Fig. 3a) (Haas et al., 2004, Ohkawa et al., 2005). Formation of the entry complex is not required for this binding, as bound ODVs were found with two different complex deficient mutants, lacking either PIF3 or PIF9 (Chapter 5). The molecular determinants of fusion are rather enigmatic as no pif mutants have been identified where fusion was totally blocked, even when binding was affected (Haas-Stapleton et al., 2004, Ohkawa et al., 2005). However, mixing of the outer leaflets of the lipid bilayers might be relatively easy due to the curvature of the microvillus membrane that partially overcomes the repellent nature of biological membranes (Wilson et al., 1998). Furthermore, this hemifusion state also results in a fluorescent signal in R18 dequenching assays with other virus mutants (Kemble et al., 1994, Laliberte et al., 2011), while R18 dequenching was considered as indicative for complete fusion in the previous studies with baculoviruses. PIF3 is important for capsid entry into the midgut cells, either by completion of the fusion process by aiding in the mixing of the inner leaflets of the lipid bilayers, or by supporting the

(a) translocation of the nucleocapsids from the ODV to the host cell cytosol (Fig. 3b) (Ohkawa et al., 2005, Chapter 5). The nucleocapsids of another complex deficient mutant, lacking PIF9, also failed to enter the midgut cell. Therefore, it is proposed for this model that the ODV entry complex mediate midgut infection by providing a scaffold to combine the PIFs that are involved in binding and fusion to facilitate the entry of nucleocapsids into epithelial midgut cells.

The infection process in the midgut is probably supported by other ODV envelope proteins, in which PIF5 and ODV-E66 have fine tuning or

(b)
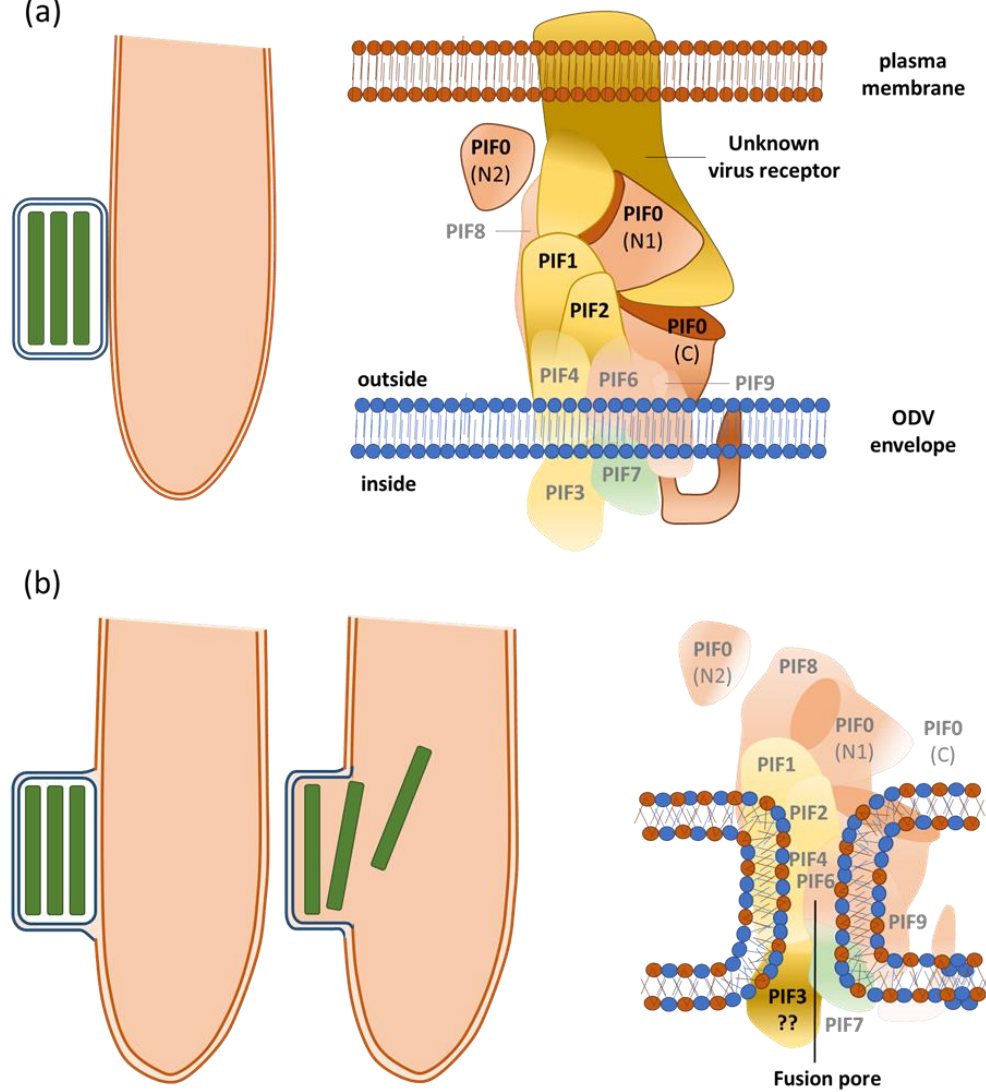

Figure 3: PIF0, 1 and 2 are important for the binding of an unknown receptor in the plasma membrane on the host cell's microvilli (a). PIF3 is important for the entry of the nucleocapsids by either completion of membrane fusion (to pass the hemifusion state) or by translocation of the capsids into the host cell (b). 
regulatory functions during midgut entry. Because of the results from the proteomic study in Chapter 7, it is tempting to speculate that these proteins regulate capsid entry by changing the stoichiometry of PIF0 and 1 in the entry complex. To create a fusion pore that is large enough to facilitate the passage of the capsids from the virion to the host cell, fusion events of multiple entry complexes might be required as it has also been shown that clustering of fusion complexes in the vaccinia virus is important for the fusion efficiency (Gray et al., 2018). Once the fusion pore is created, the actin remodelling complex in the base of the nucleocapsids possibly remodels the actin filaments inside the microvillus and clear the road to the nucleus for the first round of replication (Braunagel et al., 2001, Goley et al., 2006, Hepp et al., 2018).

\section{Concluding remarks and future perspectives}

The research presented in this thesis provides further information on the characteristics of the ODV entry complex concerning its composition (Chapter 4), important protein interactions (Chapter 6) and how the complex relates to other viral proteins in the ODV envelope (Chapter 7). It was also shown why such a complex is formed (Chapter 3) and how complex formation relates to the infectivity of ODVs (Chapter 5 and 6). The formation of such a complex complicates the interpretation of earlier research that investigated the biological function of the various PIFs as outlined in Chapter 2, since the observed phenotypes are possibly the result of secondary effects instead of being indicative for the function of that particular PIF. Furthermore, the identification of a complex-deficient mutant virus that binds and fuses with the host cell as wild type (Ohkawa et al., 2005), but nevertheless fails to establish midgut infection (The pif3 deletion mutant), already hinted to the complexity of the infection process in the larval midgut at the start of this research. Our research showed that this complex is indeed not an easy object to study as the PIFs were degraded by host proteases in pif mutant viruses (Chapter 3) and absence or minor truncation of a single component abolished complex formation (Chapter 4 and 6). This indicates that the current experimental approaches are not suitable to dissect the molecular mechanisms of the complex as there is not much room to play around without the risk of introducing secondary effects.

Studying the molecular mechanism of baculovirus oral infectivity without affecting complex formation might be accomplished by the use of nanobodies that inhibit the function of one of the components without the need of mutagenic approaches. Nanobodies are single-domain antigen binding proteins, which are derived from camelid immunoglobulins that only consists of heavy chains. Nanobodies have been used before in the structural and functional 
characterisation of fusion proteins in various human viruses as the respiratory syncytial virus and norovirus (Datalle et al., 2016, Palomo et al., 2016, Wu et al., 2017, Rossey et al., 2017, Koromyslova and Hansman, 2017). Nanobodies might also be suitable in the research to the molecular mechanism of the ODV entry complex as well as their small size (15 kDa) allows binding into small cavities in a protein (complex) surface that cannot be reached by conventional antibodies (Reviewed in Desmyter et al., 2015).

Furthermore, the study towards the molecular mechanisms of ODV midgut infection is hampered by the lack of an in vitro cell culture system, in which the alkaline conditions of the larval midgut can be simulated. The production of intestinal organoids have been shown to be useful to analyse norovirus and rotavirus infections (Yin et al., 2015, Ettayebi et al., 2016). The development of analogous systems with lepidopteran midgut cells could facilitate further studies to unravel the midgut infection process by ODVs. However, the entry of norovirus and rotavirus could be studied under cell culture conditions, while ODV entry into lepidopteran midgut cells requires highly alkaline conditions $(\mathrm{pH} 8-10)$, which are not compatible with conventional cell culturing.

Virus entry does not only require the presence of viral binding and fusion proteins, also the composition of the viral lipid membrane is important for entry into an host cell. The presence of cholesterol in the virus envelope is crucial for membrane fusion for a wide variety of viruses as the influenza virus (Sun and Whittaker, 2003), human herpesvirus 6 (Huang et al., 2006), gastroenteritis virus (Ren et al., 2008), hepatitis C virus (Aizaki et al., 2008) and dengue virus (Carro and Damonte, 2013). The ODV envelope also contain high levels of cholesterol (Braunagel and Summers, 1994). However, the importance of cholesterol for baculovirus ODV entry into midgut cells and its importance for formation of the entry complex is currently unexplored. Experiments with ODVs produced in cholesterol depleted insect cells can complement current knowledge on the requirements for the infection of midgut cells by ODVs. 


\section{References}

Ahrens, C. H., Russell, R. L. Q., Funk, C. J., Evans, J. T., Harwood, S. H., \& Rohrmann, G. F. (1997). The sequence of the Orgyia pseudotsugata multinucleocapsid nuclear polyhedrosis virus genome. Virology, 229, 381399.

Aizaki, H., Morikawa, K., Fukasawa, M., Hara, H., Inoue, Y., Tani, H., Nishijima, M., Hanada, K., Matsuura, Y., Lai, M. M., Miyamura, T., Wakita, T., \& Suzuki, T. (2008). Critical role of virion-associated cholesterol and sphingolipid in hepatitis C virus infection. Journal of Virology, 82, 5715-5724.

Atanasiu, D., Saw, W. T., Cohen, G. H., \& Eisenberg, R. J. (2010). Cascade of events governing cell-cell fusion induced by herpes simplex virus glycoproteins gD, gH/gL, and gB. Journal of Virology, 84, 12292-12299.

Biswas, S., Willis, L. G., Fang, M., Nie, Y., \& Theilmann, D. A. (2018). Autographa californica nucleopolyhedrovirus AC141 (Exon0), a potential E3 ubiquitin ligase, interacts with viral ubiquitin and AC66 to facilitate nucleocapsid egress. Journal of Virology, 92, e01713-17.

Blissard, G. W., \& Wenz, J. R. (1992). Baculovirus GP64 envelope glycoprotein is sufficient to mediate pHdependent membrane fusion. Journal of Virology, 66, 6829-6835.

Bonning, B. C., Hoover, K., Duffey, S., \& Hammock, B. D. (1995). Production of polyhedra of the Autographa californica nuclear polyhedrosis virus using the Sf21 and Tn5B1-4 cell lines and comparison with host-derived polyhedra by bioassay. Journal of Invertebrate Pathology, 66, 224-230.

Braunagel, S. C., Elton, D. M., Ma, H., \& Summers, M. D. (1996). Identification and analysis of an Autographa californica nuclear polyhedrosis virus structural protein of the occlusion-derived virus envelope: ODV-E56. Virology, 217, 97-110.

Braunagel, S. C., Guidry, P. A., Rosas-Acosta, G., Engelking, L., \& Summers, M. D. (2001). Identification of BV/ODV-C42, an Autographa californica nucleopolyhedrovirus orf101-encoded structural protein detected in infected-cell complexes with ODV-EC27 and p78/83. Journal of Virology, 75, 12331-12338.

Braunagel, S. C., Russell, W. K., Rosas-Acosta, G., Russell, D. H., \& Summers, M. D. (2003). Determination of the protein composition of the occlusion-derived virus of Autographa californica nucleopolyhedrovirus. Proceedings of the National Academy of Sciences, 100, 9797-9802.

Braunagel, S. C., \& Summers, M. D. (1994). Autographa californica nuclear polyhedrosis virus, PDV, and ECV viral envelopes and nucleocapsids: structural proteins, antigens, lipid and fatty acid profiles. Virology, 202, 315328.

Braunagel, S. C., \& Summers, M. D. (2007). Molecular biology of the baculovirus occlusion-derived virus envelope. Current Drug Targets, 8, 1084-1095.

Braunagel, S. C., Williamson, S. T., Saksena, S., Zhong, Z., Russell, W. K., Russell, D. H., \& Summers, M. D. (2004). Trafficking of ODV-E66 is mediated via a sorting motif and other viral proteins: facilitated trafficking to the inner nuclear membrane. Proceedings of the National Academy of Sciences, 101, 8372-8377.

Carro, A. C., \& Damonte, E. B. (2013). Requirement of cholesterol in the viral envelope for dengue virus infection. Virus Research, 174, 78-87.

Casartelli, M., Corti, P., Leonardi, M. G., Fiandra, L., Burlini, N., Pennacchio, F., \& Giordana, B. (2005). Absorption of albumin by the midgut of a lepidopteran larva. Journal of Insect Physiology, 51, 933-940.

Chen, H. Q., Chen, K. P., Yao, Q., Guo, Z. J., \& Wang, L. L. (2007). Characterization of a late gene, ORF67 from Bombyx mori nucleopolyhedrovirus. FEBS letters, 581, 5836-5842 
Chen, L., Hu, X., Xiang, X., Yu, S., Yang, R., \& Wu, X. (2012). Autographa californica multiple nucleopolyhedrovirus ODV-E25 (Ac94) is required for budded virus infectivity and occlusion-derived virus formation. Archives of Virology, 157, 617-625.

Chen, L., Yang, R., Hu, X., Xiang, X., Yu, S., \& Wu, X. (2013). The formation of occlusion-derived virus is affected by the expression level of ODV-E25. Virus Research, 173, 404-414.

Chen, W., Li, Z., Li, S., Li, L., Yang, K., \& Pang, Y. (2006). Identification of Spodoptera litura multicapsid nucleopolyhedrovirus ORF97, a novel protein associated with envelope of occlusion-derived virus. Virus Genes, $32,79-84$.

Ciferri, C., Chandramouli, S., Donnarumma, D., Nikitin, P. A., Cianfrocco, M. A., Gerrein, R., Feire, A. L., Barnet, S. W., Lilja, A. E., Rappuoli, R., Norais, N., Settembre, E. C., \& Carfi, A. (2015). Structural and biochemical studies of $\mathrm{HCMV} \mathrm{gH} / \mathrm{gL} / \mathrm{gO}$ and Pentamer reveal mutually exclusive cell entry complexes. Proceedings of the National Academy of Sciences, 112, 1767-1772.

Cox, J., \& Mann, M. (2008). MaxQuant enables high peptide identification rates, individualized ppb-range mass accuracies and proteome-wide protein quantification. Nature Biotechnology, 26, 1367.

Cox, J., Neuhauser, N., Michalski, A., Scheltema, R. A., Olsen, J. V., \& Mann, M. (2011). Andromeda: a peptide search engine integrated into the MaxQuant environment. Journal of Proteome Research, 10, 1794-1805.

Detalle, L., Stohr, T., Palomo, C., Piedra, P. A., Gilbert, B. E., Mas, V., Millar, A., Power, U. F., Stortelers, C., Allosery, K., Melero, J. A., \& Depla, E. (2016). Generation and characterization of ALX-0171, a potent novel therapeutic nanobody for the treatment of respiratory syncytial virus infection. Antimicrobial Agents and Chemotherapy, 60, 6-13.

Datsenko, K. A., \& Wanner, B. L. (2000). One-step inactivation of chromosomal genes in Escherichia coli K12 using PCR products. Proceedings of the National Academy of Sciences, 97, 6640-6645.

Desmyter, A., Spinelli, S., Roussel, A., \& Cambillau, C. (2015). Camelid nanobodies: killing two birds with one stone. Current Opinion in Structural Biology, 32, 1-8.

Dong, F., Wang, J., Deng, R., \& Wang, X. (2016). Autographa californica multiple nucleopolyhedrovirus gene ac81 is required for nucleocapsid envelopment. Virus Research, 221, 47-57.

Dong, Z. Q., Zhang, J., Chen, X. M., He, Q., Cao, M. Y., Wang, L., Li, H. Q., Xiao, W. F., Pan, C. X., Lu, C., \& Pan, M. H. (2014). Bombyx mori nucleopolyhedrovirus ORF79 is a per os infectivity factor associated with the PIF complex. Virus Research, 184, 62-70.

Engelhard, E. K., Kam-Morgan, L. N., Washburn, J. O., \& Volkman, L. E. (1994). The insect tracheal system: a conduit for the systemic spread of Autographa californica M nuclear polyhedrosis virus. Proceedings of the National Academy of Sciences, 91, 3224-3227

Eppstein, D. A., \& Thoma, J. A. (1975). Alkaline protease associated with the matrix protein of a virus infecting the cabbage looper. Biochemical and Biophysical Research Communications, 62, 478-484.

Ettayebi, K., Crawford, S. E., Murakami, K., Broughman, J. R., Karandikar, U., Tenge, V. R., Neil, F. H., Blutt, S. E., Zeng, X. L., Qu, L., Kou, B., Opekun, A. R., Burrin, D., Graham, D. H., Romani, S., Atmar, R. L., Estes, \& M. K., (2016). Replication of human noroviruses in stem cell-derived human enteroids. Science, 353, 1387-1393. 
Fang, M., Nie, Y., Harris, S., Erlandson, M. A., \& Theilmann, D. A. (2009). Autographa californica multiple nucleopolyhedrovirus core gene ac96 encodes a per os infectivity factor (pif-4). Journal of Virology, 83, 1256912578.

Fang, M., Nie, Y., \& Theilmann, D. A. (2009b). Deletion of the AcMNPV core gene ac109 results in budded virions that are non-infectious. Virology, 389, 66-74.

Fang, Z., Li, C., Wu, W., Yuan, M., \& Yang, K. (2016). The Autographa californica multiple nucleopolyhedrovirus Ac132 plays a role in nuclear entry. Journal of General Virology, 97, 3030-3038.

Faulkner, P., Kuzio, J., Williams, G. V., \& Wilson, J. A. (1997). Analysis of P74, a PDV envelope protein of Autographa californica nucleopolyhedrovirus required for occlusion body infectivity in vivo. Journal of General Virology, 78, 3091-3100.

Flipsen, J. T. M., van Lent, J. W. M., Goldbach, R. W., \& Vlak, J. M. (1993). Expression of polyhedrin and p10 in the midgut of AcMNPV-infected Spodoptera exigua larvae: an immunoelectron microscopic investigation. Journal of Invertebrate Pathology, 61, 17-23.

Flipsen, J. T. M., Martens, J. W. M., Van Oers, M. M., Vlak, J. M., \& Van Lent, J. W. M. (1995). Passage of Autographa californica nuclear polyhedrosis virus through the midgut epithelium of Spodoptera exigua larvae. Virology, 208, 328-335.

Garavaglia, M. J., Miele, S. A. B., Iserte, J. A., Belaich, M. N., \& Ghiringhelli, P. D. (2012). The $a c 53, a c 78$, ac101, and ac103 genes are newly discovered core genes in the family Baculoviridae. Journal of Virology, 86, 12069-12079.

Garcia-Maruniak, A., Maruniak, J. E., Zanotto, P. M., Doumbouya, A. E., Liu, J. C., Merritt, T. M., \& Lanoie, J. S. (2004). Sequence analysis of the genome of the Neodiprion sertifer nucleopolyhedrovirus. Journal of Virology, 78, 7036-7051.

Gianni, T., Amasio, M., \& Campadelli-Fiume, G. (2009). Herpes simplex virus gD forms distinct complexes with fusion executors $\mathrm{gB}$ and $\mathrm{gH} / \mathrm{gL}$ in part through the C-terminal profusion domain. Journal of Biological Chemistry, 284, 17370-17382.

Goley, E. D., Ohkawa, T., Mancuso, J., Woodruff, J. B., D'Alessio, J. A., Cande, W. Z., Volkman, L. E., \& Welch, M. D. (2006). Dynamic nuclear actin assembly by Arp2/3 complex and a baculovirus WASP-like protein. Science, 314, 464-467.

Granados, R. R., \& Lawler, K. A. (1981). In vivo pathway of Autographa californica baculovirus invasion and infection. Virology, 108, 297-308.

Gray, R., Albrecht, D., Beerli, C., Cohen, G., Henriques, R., \& Mercer, J. (2018). Nanoscale polarization of the Vaccinia virus entry fusion complex drives efficient fusion. bioRxiv, 360073

Guan, Z., Zhong, L., Li, C., Wu, W., Yuan, M., \& Yang, K. (2016). The Autographa californica multiple nucleopolyhedrovirus ac54 gene is crucial for localization of the major capsid protein VP39 at the site of nucleocapsid assembly. Journal of Virology, 90, 4115-4126.

Haas, A. L., Katzung, D. J., Reback, P. M., \& Guarino, L. A. (1996). Functional characterization of the ubiquitin variant encoded by the baculovirus Autographa californica. Biochemistry, 35, 5385-5394.

Haas-Stapleton, E. J., Washburn, J. O., \& Volkman, L. E. (2004). P74 mediates specific binding of Autographa californica M nucleopolyhedrovirus occlusion-derived virus to primary cellular targets in the midgut epithelia of Heliothis virescens larvae. Journal of Virology, 78, 6786-6791. 
Haas-Stapleton, E. J., Washburn, J. O., \& Volkman, L. E. (2005). Spodoptera frugiperda resistance to oral infection by Autographa californica multiple nucleopolyhedrovirus linked to aberrant occlusion-derived virus binding in the midgut. Journal of General Virology, 86, 1349-1355.

Harrap, K. A., Payne, C. C., \& Robertson, J. S. (1977). The properties of three baculoviruses from closely related hosts. Virology, 79, 14-31.

Harrison, R. L., Sparks, W. O., \& Bonning, B. C. (2010). Autographa californica multiple nucleopolyhedrovirus ODV-E56 envelope protein is required for oral infectivity and can be substituted functionally by Rachiplusia ou multiple nucleopolyhedrovirus ODV-E56. Journal of General Virology, 91, 1173.

Harrison, R. L., Herniou, E. A., Jehle, J. A., Theilmann, D. A., Burand, J.P., Becnel, J.J., Krell, P.J., M. van Oers, M., Mowery, J.D., Bauchan, G.R., and ICTV Report Consortium. 2019, ICTV Virus Taxonomy Profile: Baculoviridae, Journal of General Virology, 99: 1185-1186.

Hawtin, R. E., Zarkowska, T., Arnold, K., Thomas, C. J., Gooday, G. W., King, L. A., Kuzio, J. A., \& Possee, R. D. (1997). Liquefaction of Autographa californica nucleopolyhedrovirus-infected insects is dependent on the integrity of virus-encoded chitinase and cathepsin genes. Virology, 238, 243-253.

Hegedus, D., Erlandson, M. A., Gillott, C., \& Toprak, U. (2009). New insights into peritrophic matrix synthesis, architecture, and function. Annual Review of Entomology, 54, 285-302.

Hepp, S. E., Borgo, G. M., Ticau, S., Ohkawa, T., \& Welch, M. D. (2018). Baculovirus AC102 is a nucleocapsid protein that is crucial for nuclear actin polymerization and nucleocapsid morphogenesis. Journal of Virology, 92, e00111-18.

Hoekstra, D., De Boer, T., Klappe, K., \& Wilschut, J. (1984). Fluorescence method for measuring the kinetics of fusion between biological membranes. Biochemistry, 23, 5675-5681.

Hopkins, R. F., \& Esposito, D. (2009). A rapid method for titrating baculovirus stocks using the Sf-9 Easy Titer cell line. Biotechniques, 47, 785-788.

Horton, H. M., \& Burand, J. P. (1993). Saturable attachment sites for polyhedron-derived baculovirus on insect cells and evidence for entry via direct membrane fusion. Journal of Virology, 67, 1860-1868.

Hou, D., Kuang, W., Luo, S., Zhang, F., Zhou, F., Chen, T., Zhang, Y., Wang, H., Hu, Z., Deng, F., \& Wang, M. (2019). Baculovirus ODV-E66 degrades larval peritrophic membrane to facilitate baculovirus oral infection. Virology, 537, 157-167

Huang, H., Li, Y., Sadaoka, T., Tang, H., Yamamoto, T., Yamanishi, K., \& Mori, Y. (2006). Human herpesvirus 6 envelope cholesterol is required for virus entry. Journal of General Virology, 87, 277-285.

Huang, H., Wang, M., Deng, F., Wang, H., \& Hu, Z. (2012). ORF85 of HearNPV encodes the per os infectivity factor 4 (PIF4) and is essential for the formation of the PIF complex. Virology, 427, 217-223.

Hubner, N. C., Bird, A. W., Cox, J., Splettstoesser, B., Bandilla, P., Poser, I., Hyman, A., \& Mann, M. (2010). Quantitative proteomics combined with BAC Transgene Omics reveals in vivo protein interactions. The Journal of Cell Biology, 189, 739-754.

Javed, M. A., Harris, S., Willis, L. G., Theilmann, D. A., Donly, B. C., Erlandson, M. A., \& Hegedus, D. D. (2016). Microscopic investigation of AcMNPV infection in the Trichoplusia ni midgut. Journal of Invertebrate Pathology, 141, 24-33. 
Javed, M. A., Biswas, S., Willis, L. G., Harris, S., Pritchard, C., van Oers, M. M., \& Theilmann, D. A. (2017). Autographa californica multiple nucleopolyhedrovirus AC83 is a per os infectivity factor (PIF) protein required for occlusion-derived virus (ODV) and budded virus nucleocapsid assembly as well as assembly of the PIF complex in ODV envelopes. Journal of Virology, 91, e02115-16.

Javed, M. A., Coutu, C., Theilmann, D. A., Erlandson, M. A., \& Hegedus, D. D. (2019). Proteomics analysis of Trichoplusia ni midgut epithelial cell brush border membrane vesicles. Insect Science, 26, 424-440.

Jehle, J. A., Blissard, G. W., Bonning, B. C., Cory, J. S., Herniou, E. A., Rohrmann, G. F., Theilmann, D. A., Thiem, S. M., \& Vlak, J. M. (2006). On the classification and nomenclature of baculoviruses: a proposal for revision. Archives of Virology, 151, 1257-1266.

Ji, X., Sutton, G., Evans, G., Axford, D., Owen, R., \& Stuart, D. I. (2010). How baculovirus polyhedra fit square pegs into round holes to robustly package viruses. The EMBO Journal, 29, 505-514.

Jiao, Y., Wang, J., Deng, R., Yu, X., \& Wang, X. (2019). AcMNPV-miR-3 is a miRNA encoded by Autographa californica nucleopolyhedrovirus and regulates the viral infection by targeting ac101. Virus Research, 267, 49-58.

Kabanova, A., Marcandalli, J., Zhou, T., Bianchi, S., Baxa, U., Tsybovsky, Y., Lilleri, D., Silacci-Fregni, C., Foglierini, M., Fernandez-Rodriguez, B. M., Druz, A., Zhang, B., Geiger, R., Pagani, M., Sallusto, F., Kwong, P. D., Corti, D., Lanzavecchia, A., \& Perez, L., (2016). Platelet-derived growth factor- $\alpha$ receptor is the cellular receptor for human cytomegalovirus gHgLgO trimer. Nature Microbiology, 1, 16082.

Käll, L., Krogh, A., \& Sonnhammer, E. L. (2004). A combined transmembrane topology and signal peptide prediction method. Journal of Molecular Biology, 338, 1027-1036.

Kariithi, H. M., Ince, I. A., Boeren, S., Vervoort, J., Bergoin, M., van Oers, M. M., Abd-Alla, A. M. M., \& Vlak, J. M. (2010). Proteomic analysis of Glossina pallidipes salivary gland hypertrophy virus virions for immune intervention in tsetse fly colonies. Journal of General Virology, 91, 3065-3074.

Kawanishi, C. Y., Summers, M. D., Stoltz, D. B., \& Arnott, H. J. (1972). Entry of an insect virus in vivo by fusion of viral envelope and microvillus membrane. Journal of Invertebrate Pathology, 20, 104-108.

Ke, J., Wang, J., Deng, R., \& Wang, X. (2008). Autographa californica multiple nucleopolyhedrovirus ac66 is required for the efficient egress of nucleocapsids from the nucleus, general synthesis of preoccluded virions and occlusion body formation. Virology, 374, 421-431.

Kemble, G. W., Danieli, T., \& White, J. M. (1994). Lipid-anchored influenza hemagglutinin promotes hemifusion, not complete fusion. Cell, 76, 383-391.

Kikhno, I., Gutiérrez, S., Croizier, L., Croizier, G., \& Ferber, M. L. (2002). Characterization of pif, a gene required for the per os infectivity of Spodoptera littoralis nucleopolyhedrovirus. Journal of General Virology, 83, 3013-3022.

Koromyslova, A. D., \& Hansman, G. S. (2017). Nanobodies targeting norovirus capsid reveal functional epitopes and potential mechanisms of neutralization. PLoS pathogens, 13, e1006636.

Kuzio, J., Jaques, R. T., \& Faulkner, P. (1989). Identification of $p 74$, a gene essential for virulence of baculovirus occlusion bodies. Virology, 173, 759-763.

Lai, Q., Wu, W., Li, A., Wang, W., Yuan, M., \& Yang, K. (2018). The 38K-mediated specific dephosphorylation of the viral core protein P6.9 plays an important role in the nucleocapsid assembly of Autographa californica multiple nucleopolyhedrovirus. Journal of Virology, 92, e01989-17. 
Laliberte, J. P., Weisberg, A. S., \& Moss, B. (2011). The membrane fusion step of Vaccinia virus entry is cooperatively mediated by multiple viral proteins and host cell components. PLoS pathogens, 7, e1002446.

Lanier, L. M., Slack, J. M., \& Volkman, L. E. (1996). Actin binding and proteolysis by the baculovirus AcMNPV: the role of virion-associated V-CATH. Virology, 216, 380-388.

Lapointe, R., Popham, H. J., Straschil, U., Goulding, D., O'Reilly, D. R., \& Olszewski, J. A. (2004). Characterization of two Autographa californica nucleopolyhedrovirus proteins, AC145 and AC150, which affect oral infectivity in a host-dependent manner. Journal of Virology, 78, 6439-6448.

Lehiy, C. J., Wu, W., Berretta, M. F., \& Passarelli, A. L. (2013). Autographa californica M nucleopolyhedrovirus open reading frame 109 affects infectious budded virus production and nucleocapsid envelopment in the nucleus of cells. Virology, 435, 442-452.

van Lent, J. W. M., Groenen, J. T. M., Klinge-Roode, E. C., Rohrmann, G. F., Zuidema, D., \& Vlak, J. M. (1990). Localization of the $34 \mathrm{kDa}$ polyhedron envelope protein in Spodoptera frugiperda cells infected with Autographa california nuclear polyhedrosis virus. Archives of Virology, 111, 103-114.

Li, G., Wang, J., Deng, R., \& Wang, X. (2008). Characterization of AcMNPV with a deletion of ac68 gene. Virus Genes, 37, 119-127.

Li, S., Li, L., Zhao, H., \& Liu, W. (2018). Disruption of Autographa californica multiple nucleopolyhedrovirus ac111 results in reduced per os infectivity in a host-dependent manner. Viruses, 10, 527.

Li, X., Song, J., Jiang, T., Liang, C., \& Chen, X. (2007). The N-terminal hydrophobic sequence of Autographa californica nucleopolyhedrovirus PIF-3 is essential for oral infection. Archives of Virology, 152, 1851-1858.

Li, Y., Shen, S., Hu, L., Deng, F., Vlak, J. M., Hu, Z., Wang, H., \& Wang, M. (2018b). The functional oligomeric state of tegument protein GP41 is essential for baculovirus budded virion and occlusion-derived virion assembly. Journal of Virology, 92, e02083-17.

Li, Z., Li, C., Yang, K., Wang, L., Yin, C., Gong, Y., \& Pang, Y. (2003). Characterization of a chitin-binding protein GP37 of Spodoptera litura multicapsid nucleopolyhedrovirus. Virus Research, 96, 113-122.

Liang, C., Lan, D., Zhao, S., Liu, L., Xue, Y., Zhang, Y., Wang, Y., \& Chen, X. (2015). The AC124 protein is not essential for the propagation of Autographa californica multiple nucleopolyhedrovirus, but it is a viral pathogenicity factor. Archives of Virology, 160, 275-284.

Lin, L., Wang, J., Deng, R., Ke, J., Wu, H., \& Wang, X. (2009). AC109 is required for the nucleocapsid assembly of Autographa californica multiple nucleopolyhedrovirus. Virus Research, 144, 130-135.

Liu, X., Chen, K., Cai, K., \& Yao, Q. (2008). Determination of protein composition and host-derived proteins of Bombyx mori nucleopolyhedrovirus by 2-dimensional electrophoresis and mass spectrometry. Intervirology, 51, 369-376.

Liu, X., Ma, X., Lei, C., Xiao, Y., Zhang, Z., \& Sun, X. (2011). Synergistic effects of Cydia pomonella granulovirus GP37 on the infectivity of nucleopolyhedroviruses and the lethality of Bacillus thuringiensis. Archives of Virology, 156, 1707-1715.

Liu, X., Zhao, H., Fang, Z., Yuan, M., Yang, K., \& Pang, Y. (2012). Distribution and phosphorylation of the basic protein P6.9 of Autographa californica nucleopolyhedrovirus. Journal of Virology, 86, 12217-12227. 
Liu, J., Zhu, L., Zhang, S., Deng, Z., Huang, Z., Yuan, M., Wu, W., \& Yang, K. (2016). The Autographa californica multiple nucleopolyhedrovirus acl10 gene encodes a new per os infectivity factor. Virus Research, $221,30-37$.

Liu, X., Fang, W., Fan, R., Zhang, L., Lei, C., Zhang, J., Nian, W., Dou, T., An, S., \& Sun, X. (2019). Granulovirus GP37 Facilitated ODVs cross insect peritrophic membranes and fuse with epithelia. Toxins, 11, 145.

Luckow, V. A., Lee, S. C., Barry, G. F., \& Olins, P. O. (1993). Efficient generation of infectious recombinant baculoviruses by site-specific transposon-mediated insertion of foreign genes into a baculovirus genome propagated in Escherichia coli. Journal of Virology, 67, 4566-4579.

Luo, S., Zhang, Y., Xu, X., Westenberg, M., Vlak, J. M., Wang, H., Hu, Z., \& Deng, F. (2011). Helicoverpa armigera nucleopolyhedrovirus occlusion-derived virus-associated protein, HA100, affects oral infectivity in vivo but not virus replication in vitro. Journal of General Virology, 92, 1324-1331.

Luo, X. C., Wang, S. S., Zhang, J., Qian, D. D., Wang, S. M., \& Li, L. L. (2013). Effects of early or overexpression of the Autographa californica multiple nucleopolyhedrovirus orf94 (ODV-E25) on virus replication. PloS One, 8 , e65635.

Marek, M., Merten, O. W., Galibert, L., Vlak, J. M., \& van Oers, M. M. (2011). Baculovirus VP80 protein and the F-actin cytoskeleton interact and connect the viral replication factory with the nuclear periphery. Journal of Virology, 85, 5350-5362.

Mettenmeyer, A. (2002). Viral insecticides hold promise forbio-control. Farming Ahead, 124: 50 - 1.

Monsma, S. A., \& Blissard, G. W. (1995). Identification of a membrane fusion domain and an oligomerization domain in the baculovirus GP64 envelope fusion protein. Journal of Virology, 69, 2583-2595.

Moscardi, F. (2007) A nucleopolyhedrovirus for control of the velvetbean caterpillar in brazilian soybeans. Biological Control: A Global Perspective, 344-352, ISBN 184-593-266-8.

Moscardi, F., \& Sosa-Gomez, D. R. (1992). Use of viruses against soybean caterpillars in Brazil. In Pest management in soybean (pp. 98-109). Springer, Dordrecht.

Moss, B. (2016). Membrane fusion during poxvirus entry. Seminars in Cell \& Developmental Biology, 60, 89-96, Academic Press.

Mu, J., van Lent, J. W. M., Smagghe, G., Wang, Y., Chen, X., Vlak, J. M., \& van Oers, M. M. (2014). Live imaging of baculovirus infection of midgut epithelium cells: a functional assay of per os infectivity factors. Journal of General Virology, 95, 2531-2539.

Nie, Y., Fang, M., Erlandson, M. A., \& Theilmann, D. A. (2012). Analysis of the Autographa californica multiple nucleopolyhedrovirus overlapping gene pair lef3 and ac68 reveals that AC68 is a per os infectivity factor and that LEF3 is critical, but not essential, for virus replication. Journal of Virology, 86, 3985-3994.

Nie, Y., Fang, M., \& Theilmann, D. A. (2011). Autographa californica multiple nucleopolyhedrovirus core gene ac92 (P33) is required for efficient budded virus production. Virology, 409, 38-45.

Ohkawa, T., Volkman, L. E., \& Welch, M. D. (2010). Actin-based motility drives baculovirus transit to the nucleus and cell surface. The Journal of Cell Biology, 190, 187-195.

Ohkawa, T., Washburn, J. O., Sitapara, R., Sid, E., \& Volkman, L. E. (2005). Specific binding of Autographa californica M nucleopolyhedrovirus occlusion-derived virus to midgut cells of Heliothis virescens larvae is mediated by products of pif genes ac119 and ac022 but not by ac115. Journal of Virology, 79, 15258-15264. 
Olszewski, J., \& Miller, L. K. (1997a). Identification and characterization of a baculovirus structural protein, VP1054, required for nucleocapsid formation. Journal of Virology, 71, 5040-5050.

Olszewski, J., \& Miller, L. K. (1997b). A role for baculovirus GP41 in budded virus production. Virology, 233, 292-301.

Ono, C., Kamagata, T., Taka, H., Sahara, K., Asano, S. I., \& Bando, H. (2012). Phenotypic grouping of 141 BmNPVs lacking viral gene sequences. Virus Research, 165, 197-206.

Palomo, C., Mas, V., Detalle, L., Depla, E., Cano, O., Vázquez, M., Stortelers, C., \& Melero, J. A. (2016). Trivalency of a nanobody specific for the Human respiratory syncytial virus fusion glycoprotein drastically enhances virus neutralization and impacts escape mutant selection. Antimicrobial Agents and Chemotherapy, 60, 6498-6509.

Payne, C. C., \& Kalmakoff, J. (1978). Alkaline protease associated with virus particles of a nuclear polyhedrosis virus: assay, purification, and properties. Journal of Virology, 26, 84-92.

Peng, K., van Oers, M. M., Hu, Z., van Lent, J. W., \& Vlak, J. M. (2010). Baculovirus per os infectivity factors form a complex on the surface of occlusion-derived virus. Journal of Virology, 84, 9497-9504.

Peng, K., Wu, M., Deng, F., Song, J., Dong, C., Wang, H., \& Hu, Z. (2010b). Identification of protein-protein interactions of the occlusion-derived virus-associated proteins of Helicoverpa armigera nucleopolyhedrovirus. Journal of General Virology, 91, 659-670.

Peng, K., van Lent, J. W. M., Vlak, J. M., Hu, Z., \& van Oers, M. M. (2011). In situ cleavage of baculovirus occlusion-derived virus receptor binding protein P74 in the peroral infectivity complex. Journal of Virology, 85, 10710-10718.

Peng, K., van Lent, J. W. M., Boeren, S., Fang, M., Theilmann, D. A., Erlandson, M. A., Vlak, J. M., \& van Oers, M. M. (2012). Characterization of novel components of the baculovirus per os infectivity factor complex. Journal of Virology, 86, 4981-4988.

Pijlman, G. P., Pruijssers, A. J., \& Vlak, J. M. (2003). Identification of pif-2, a third conserved baculovirus gene required for per os infection of insects. Journal of General Virology, 84, 2041-2049.

Ren, X., Glende, J., Yin, J., Schwegmann-Wessels, C., \& Herrler, G. (2008). Importance of cholesterol for infection of cells by transmissible gastroenteritis virus. Virus Research, 137, 220-224.

Rohrmann, G. F. (2013). The baculovirus replication cycle: Effects on cells and insects. In Baculovirus Molecular Biology. 3rd edition. National Center for Biotechnology Information, Bethesda (US).

Ros, V. I., van Houte, S., Hemerik, L., \& van Oers, M. M. (2015). Baculovirus-induced tree-top disease: how extended is the role of egt as a gene for the extended phenotype?. Molecular Ecology, 24, 249-258.

Rossey, I., Gilman, M. S., Kabeche, S. C., Sedeyn, K., Wrapp, D., Kanekiyo, M., Chen, M., Mas, V., Spitaels, J., Melero, J. A., Graham, B. S., Schepens, B., McLellan, J. S., \& Saelens, X. (2017). Potent single-domain antibodies that arrest respiratory syncytial virus fusion protein in its prefusion state. Nature Communications, 8 , 14158.

Russell, R. L. Q., Funk, C. J., \& Rohrmann, G. F. (1997). Association of a baculovirus-encoded protein with the capsid basal region. Virology, 227, 142-152.

Russell, R. L. Q., \& Rohrmann, G. F. (1993). A 25-kDa protein is associated with the envelopes of occluded baculovirus virions. Virology, 195, 532-540. 
Russell, R. L. Q., \& Rohrmann, G. F. (1997). Characterization of P91, a Protein Associated with Virions of an Orgyia pseudotsugata Baculovirus. Virology, 233, 210-223.

Ryckman, B. J., Jarvis, M. A., Drummond, D. D., Nelson, J. A., \& Johnson, D. C. (2006). Human cytomegalovirus entry into epithelial and endothelial cells depends on genes UL128 to UL150 and occurs by endocytosis and low-pH fusion. Journal of Virology, 80, 710-722.

Sathiyamoorthy, K., Chen, J., Longnecker, R., \& Jardetzky, T. S. (2017). The complexity in herpesvirus entry. Current Opinion in Virology, 24, 97-104.

Shi, S. L., Pan, M. H., \& Lu, C. (2007). Characterization of Antheraea pernyi nucleopolyhedrovirus $p 11$ gene, a homologue of Autographa californica nucleopolyhedrovirus orf108. Virus Genes, 35, 97-101.

Shao, W., He, L., Chen, Q., Li, J., Deng, F., Wang, H., Hu, Z., \& Wang, M. (2019). Functional Characterization of the Group I Alphabaculovirus Specific Gene ac73. Virologica Sinica, 1-11.

Shi, Y., Li, K., Tang, P., Li, Y., Zhou, Q., Yang, K., \& Zhang, Q. (2015). Three-dimensional visualization of the Autographa californica multiple nucleopolyhedrovirus occlusion-derived virion envelopment process gives new clues as to its mechanism. Virology, 476, 298-303.

Simón, O., Palma, L., Williams, T., López-Ferber, M., \& Caballero, P. (2012). Analysis of a naturallyoccurring deletion mutant of Spodoptera frugiperda multiple nucleopolyhedrovirus reveals $s f 58$ as a new per os infectivity factor of lepidopteran-infecting baculoviruses. Journal of Invertebrate Pathology, 109, 117-126.

Slack, J. M., Dougherty, E. M., \& Lawrence, S. D. (2001). A study of the Autographa californica multiple nucleopolyhedrovirus ODV envelope protein P74 using a GFP tag. Journal of General Virology, 82, 2279-2287.

Slack, J. M., \& Lawrence, S. D. (2005). Evidence for proteolytic cleavage of the baculovirus occlusion-derived virion envelope protein P74. Journal of General Virology, 86, 1637-1643.

Slack, J. M., Lawrence, S. D., Krell, P. J., \& Arif, B. M. (2008). Trypsin cleavage of the baculovirus occlusionderived virus attachment protein P74 is prerequisite in per os infection. Journal of General Virology, 89, 23882397.

Slack, J. M., Lawrence, S. D., Krell, P. J., \& Arif, B. M. (2010). A soluble form of P74 can act as a per os infectivity factor to the Autographa californica multiple nucleopolyhedrovirus. Journal of General Virology, 91, 915-918

Slavicek, J. M. (2012). Baculovirus enhancins and their role in viral pathogenicity. Molecular Virology, 147-168.

Smith, G. E., \& Summers, M. D. (1978). Analysis of baculovirus genomes with restriction endonucleases. Virology, 89, 517-527.

Song, J., Wang, R., Deng, F., Wang, H., \& Hu, Z. (2008). Functional studies of per os infectivity factors of Helicoverpa armigera single nucleocapsid nucleopolyhedrovirus. Journal of General Virology, 89, 2331-2338.

Song, J., Wang, M., Huang, H., Luo, X., Deng, F., Wang, H., \& Hu, Z. (2012). Functional studies of per os infectivity factor 3 of Helicoverpa armigera nucleopolyhedrovirus. Journal of General Virology, 93, 374-382.

Song, J., Wang, X., Hou, D., Huang, H., Liu, X., Deng, F., Wang, H., Arif, B. M., Hu, Z., \& Wang, M. (2016). The host specificities of baculovirus per os infectivity factors. PloS one, 11, e0159862. 
Sparks, W. O., Harrison, R. L., \& Bonning, B. C. (2011a). Autographa californica multiple nucleopolyhedrovirus ODV-E56 is a per os infectivity factor, but is not essential for binding and fusion of occlusion-derived virus to the host midgut. Virology, 409, 69-76.

Sparks, W. O., Rohlfing, A., \& Bonning, B. C. (2011b). A peptide with similarity to baculovirus ODV-E66 binds the gut epithelium of Heliothis virescens and impedes infection with Autographa californica multiple nucleopolyhedrovirus. Journal of General Virology, 92, 1051-1060.

Summers, M. D., \& Smith, G. E. (1975). Trichoplusia ni granulosis virus granulin: a phenol-soluble, phosphorylated protein. Journal of Virology, 16, 1108-1116.

Sun, X., \& Whittaker, G. R. (2003). Role for influenza virus envelope cholesterol in virus entry and infection. Journal of Virology, 77, 12543-12551.

Suzuki, N., Nonaka, H., Tsuge, Y., Inui, M., \& Yukawa, H. (2005). New multiple-deletion method for the Corynebacterium glutamicum genome, using a mutant lox sequence. Applied Environmental Microbiololy, 71, 8472-8480.

Szewczyk, B., Rabalski, L., Krol, E., Sihler, W., \& de Souza, M. L. (2009). Baculovirus biopesticides-safe alternative to chemical protection of plants. Embrapa Recursos Genéticos e Biotecnologia-Artigo em periódico indexado (ALICE).

Tang, Q., Li, G., Yao, Q., Chen, L., Lv, P., Lian, C., \& Chen, K. (2013). BM91 is an envelope component of ODV but is dispensable for the propagation of Bombyx mori nucleopolyhedrovirus. Journal of Invertebrate Pathology, 113, 70-77.

Terra, W. R., \& Ferreira, C. (1994). Insect digestive enzymes: properties, compartmentalization and function. Comparative Biochemistry and Physiology Part B: Comparative Biochemistry, 109, 1-62.

Thézé, J., Bézier, A., Periquet, G., Drezen, J. M., \& Herniou, E. A. (2011). Paleozoic origin of insect large dsDNA viruses. Proceedings of the National Academy of Sciences, 108, 15931-15935.

Toprak, U., Harris, S., Baldwin, D., Theilmann, D. A., Gillott, C., Hegedus, D. D., \& Erlandson, M. A. (2012). Role of enhancin in Mamestra configurata nucleopolyhedrovirus virulence: selective degradation of host peritrophic matrix proteins. Journal of General Virology, 93, 744-753.

Tweeten, K. A., Bulla, L. A., \& Consigli, R. A. (1978). Characterization of an alkaline protease associated with a granulosis virus of Plodia interpunctella. Journal of Virology, 26, 702-711.

Vanarsdall, A. L., Chase, M. C., \& Johnson, D. C. (2011). Human cytomegalovirus glycoprotein gO complexes with $\mathrm{gH} / \mathrm{gL}$, promoting interference with viral entry into human fibroblasts but not entry into epithelial cells. Journal of Virology, 85, 11638-11645.

Vialard, J. E., Yuen, L., \& Richardson, C. D. (1990). Identification and characterization of a baculovirus occlusion body glycoprotein which resembles spheroidin, an entomopoxvirus protein. Journal of Virology, 64, 5804-5811.

Vialard, J. E., \& Richardson, C. D. (1993). The 1,629-nucleotide open reading frame located downstream of the Autographa californica nuclear polyhedrosis virus polyhedrin gene encodes a nucleocapsid-associated phosphoprotein. Journal of Virology, 67, 5859-5866.

Wang, M., Tuladhar, E., Shen, S., Wang, H., van Oers, M. M., Vlak, J. M., \& Westenberg, M. (2010b). Specificity of baculovirus P6.9 basic DNA-binding proteins and critical role of the $\mathrm{C}$ terminus in virion formation. Journal of Virology, 84, 8821-8828. 
Wang, P., \& Granados, R. R. (1997). An intestinal mucin is the target substrate for a baculovirus enhancin. Proceedings of the National Academy of Sciences, 94, 6977-6982.

Wang, R., Deng, F., Hou, D., Zhao, Y., Guo, L., Wang, H., \& Hu, Z. (2010). Proteomics of the Autographa californica nucleopolyhedrovirus budded virions. Journal of Virology, 84, 7233-7242.

Wang, X., Chen, C., Zhang, N., Li, J., Deng, F., Wang, H., Vlak, J. M., Hu, Z., \& Wang, M. (2018). The group I alphabaculovirus-specific protein, AC5, is a novel component of the occlusion body but is not associated with ODVs or the PIF complex. Journal of General Virology, 99, 585-595.

Wang, X., Liu, X., Makalliwa, G. A., Li, J., Wang, H., Hu, Z., \& Wang, M. (2017). Per os infectivity factors: a complicated and evolutionarily conserved entry machinery of baculovirus. Science China Life Sciences, 60, 806815.

Wang, Y., Grenet, A. G., Castelli, I., Cermenati, G., Ravallec, M., Fiandra, L., Debaisieux, S., Multeau, C., Lautredou, N., Li, Y., Casartelli, M., \& Ogliastro, M., (2013). Densovirus crosses the insect midgut by transcytosis and disturbs the epithelial barrier function. Journal of Virology, 87, 12380-12391.

Wang, Y., Wu, W., Li, Z., Yuan, M., Feng, G., Yu, Q., Yang, K., \& Pang, Y. (2007). ac18 is not essential for the propagation of Autographa californica multiple nucleopolyhedrovirus. Virology, 367, 71-81.

Washburn, J. O., Chan, E. Y., Volkman, L. E., Aumiller, J. J., \& Jarvis, D. L. (2003). Early synthesis of budded virus envelope fusion protein GP64 enhances Autographa californica multicapsid nucleopolyhedrovirus virulence in orally infected Heliothis virescens. Journal of Virology, 77, 280-290.

Wei, W., Zhou, Y., Lei, C., \& Sun, X. (2012). Autographa californica multiple nucleopolyhedrovirus orf114 is not essential for virus replication in vitro, but its knockout reduces per os infectivity in vivo. Virus Genes, 45, 360369.

Westenberg, M., Soedling, H. M., Mann, D. A., Nicholson, L. J., \& Dolphin, C. T. (2010). Counter-selection recombineering of the baculovirus genome: a strategy for seamless modification of repeat-containing BACs. Nucleic Acids Research, 38, e166-e166.

Whitford, M., \& Faulkner, P. (1992a). Nucleotide sequence and transcriptional analysis of a gene encoding GP41, a structural glycoprotein of the baculovirus Autographa californica nuclear polyhedrosis virus. Journal of Virology, 66, 4763-4768.

Whitford, M., \& Faulkner, P. (1992b). A structural polypeptide of the baculovirus Autographa californica nuclear polyhedrosis virus contains O-linked N-acetylglucosamine. Journal of Virology, 66, 3324-3329.

Whitt, M. A., \& Manning, J. S. (1988). A phosphorylated 34-kDa protein and a subpopulation of polyhedrin are thiol linked to the carbohydrate layer surrounding a baculovirus occlusion body. Virology, 163, 33-42.

Wood, H. A. (1980). Protease degradation of Autographa californica nuclear polyhedrosis virus proteins. Virology, 103, 392-399.

Williams, G. V., \& Faulkner, P. (1997). Cytological changes and viral morphogenesis during baculovirus infection. In: The Baculoviruses (pp. 61-107). Springer, Boston, MA.

Williams, T., Bergoin, M., \& van Oers, M. M. (2017). Diversity of large DNA viruses of invertebrates. Journal of Invertebrate Pathology, 147, 4-22.

Wilson, N. F., \& Snell, W. J. (1998). Microvilli and cell-cell fusion during fertilization. Trends in Cell Biology, 8, 93-96. 
Wu, W., \& Passarelli, A. L. (2010). Autographa californica multiple nucleopolyhedrovirus AC92 (ORF92, P33) is required for budded virus production and multiply enveloped occlusion-derived virus formation. Journal of Virology, 84, 12351-12361.

Wu, Y., Jiang, S., \& Ying, T. (2017). Single-domain antibodies as therapeutics against human viral diseases. Frontiers in Immunology, 8, 1802.

Xiang, X., Chen, L., Hu, X., Yu, S., Yang, R., \& Wu, X. (2011). Autographa californica multiple nucleopolyhedrovirus ODV-E66 is an essential gene required for oral infectivity. Virus Research, 158, 72-78.

Xu, H. J., Yang, Z. N., Zhao, J. F., Tian, C. H., Ge, J. Q., Tang, X. D., Bao, Y. Y., \& Zhang, C. X. (2008). Bombyx mori nucleopolyhedrovirus ORF56 encodes an occlusion-derived virus protein and is not essential for budded virus production. Journal of General Virology, 89, 1212-1219.

Yao, L., Zhou, W., Xu, H., Zheng, Y., \& Qi, Y. (2004). The Heliothis armigera single nucleocapsid nucleopolyhedrovirus envelope protein P74 is required for infection of the host midgut. Virus Research, 104, 111121.

Yin, Y., Bijvelds, M., Dang, W., Xu, L., van der Eijk, A. A., Knipping, K., Tuysuz, N., Dekkers, J. F., Wang, Y., de Jonge, J., Sprengers, D., van der Laan, L. J. W., Beekman, J. M., ten Berge, D., Metselaar, H. J., de Jonge, H., Koopmans, M. P. G., Peppelenbosch, M. P., Pan, Q., (2015). Modeling rotavirus infection and antiviral therapy using primary intestinal organoids. Antiviral Research, 123, 120-131.

Zhang, J. H., Ohkawa, T., Washburn, J. O., \& Volkman, L. E. (2005). Effects of AC150 on virulence and pathogenesis of Autographa californica multiple nucleopolyhedrovirus in noctuid hosts. Journal of General Virology, 86, 1619-1627.

Zheng, Q., Shen, Y., Kon, X., Zhang, J., Feng, M., \& Wu, X. (2017). Protein-protein interactions of the baculovirus per os infectivity factors (PIFs) in the PIF complex. Journal of General Virology, 98, 853-861.

Zhu, S., Wang, W., Wang, Y., Yuan, M., \& Yang, K. (2013). The baculovirus core gene ac83 is required for nucleocapsid assembly and per os infectivity of Autographa californica nucleopolyhedrovirus. Journal of Virology, 87, 10573-10586. 


\section{List of abbreviations}

Viruses

AcMNPV

AgMNPV

BmNPV

CMV

CuniNPV

HearMNPV

SfMNPV

SpltNPV

\section{General}

$\begin{array}{ll}\text { ABC } & \text { ammonium bicarbonate } \\ \text { AP-buffer } & \text { alkaline phosphatase buffer } \\ \text { BBMV } & \text { brush border membrane vesicle } \\ \text { BiFC } & \text { bimolecular fluorescence protein complementation assay } \\ \text { BN-PAGE } & \text { blue native-PAGE } \\ \text { bp } & \text { base pair } \\ \text { BV } & \text { budded virus } \\ \text { cat } & \text { chloramphenicol acetyl transferase resistance gene } \\ \text { CBD } & \text { chitin-binding domain } \\ \text { C-OB } & \text { cell culture-derived OB } \\ \text { C-ODV } & \text { cell culture-derived ODV } \\ \text { CoIP } & \text { co-immunoprecipitation } \\ \text { del } & \text { deletion } \\ \text { dpi } & \text { influenza virus hemagglutinin tag } \\ \text { dpt } & \text { end point dilution assay } \\ \text { DTT } & \text { days post transfection } \\ \text { EDTA } & \text { ethylenediaminetetraacetic acid } \\ \text { eGFP } & \text { enhanced green fluorescent protein } \\ \text { EPDA } & \text { elys }\end{array}$

Autographa californica multiple nucleopolyhedrovirus Anticarsia gemmatalis multiple nucleopolyhedrovirus Bombyx mori nucleopolyhedrovirus Cytomegalovirus Culex nigripalpus nucleopolyhedrovirus Helicoverpa armigera multiple nucleopolyhedrovirus Spodoptera frugiperda multiple nucleopolyhedrovirus Spodoptera litura nucleopolyhedrovirus 


\begin{tabular}{|c|c|}
\hline INM-SM & inner nuclear membrane sorting motif \\
\hline $\mathrm{kDa}$ & kilo dalton \\
\hline LC-MS/MS & liquid chromatography mass spectrometry \\
\hline $\mathrm{L}-\mathrm{OB}$ & larva-derived OB \\
\hline L-ODV & larva-derived ODV \\
\hline MNPV & multiple nucleopolyhedrovirus \\
\hline MOI & multiplicity of infection \\
\hline mut & mutant \\
\hline $\mathrm{nt}$ & nucleotide \\
\hline OB & occlusion body \\
\hline ODV & occlusion-derived virus \\
\hline ORF & open reading frame \\
\hline $\mathrm{pFBD}$ & pfast bac dual plasmid \\
\hline PIF & per os infectivity factor \\
\hline Polh & polyhedrin \\
\hline $\mathrm{R} 18$ & octadecyl rhodamine \\
\hline rep & repair \\
\hline SDS & sodium dodecyl sulphate \\
\hline SNPV & single nucleopolyhedrovirus \\
\hline $\mathrm{TCID}_{50}$ & tissue culture infective dose of $50 \%$ \\
\hline VP39 & viral protein of $39 \mathrm{kDa}$, the baculovirus major capsid protein \\
\hline $\mathrm{Wt}$ & wild type \\
\hline $\mathrm{w} / \mathrm{v}$ & weight per volume \\
\hline $\mathrm{w} / \mathrm{w}$ & weight per weight \\
\hline $\mathrm{Y} 2 \mathrm{H}$ & yeast-two hybrid assay \\
\hline ZF-domain & zinc finger domain \\
\hline
\end{tabular}




\section{Summary}

Insect larvae are infected by baculoviruses when they consume plant material that is contaminated with occlusion bodies (OBs). OBs are polyhedral shaped particles of crystallized protein (polyhedrin or granulin), in which the occlusion derived viruses (ODVs) are embedded. Upon ingestion of the OBs, the ODVs are released in the alkaline lumen of the midgut and infect the midgut epithelial cells. To be infectious for these cells under alkaline conditions, the ODV envelope contains a set of proteins, which are called per os infectivity factors or PIFs. Eight different PIFs had been identified at the start of this research, of which six were known to participate in the formation of a large complex: the ODV entry complex. Presently, ten different PIFs are known, of which nine form the entry complex. This complex has a stable core that is formed by PIF1 to 4, which even resists treatment with denaturing and reducing agents (SDS and $\beta$-mercaptoethanol), and five additional PIFs (PIF0 and PIF6 to 9), which are associated to the complex more loosely. PIF5 is the only PIF-protein that is not a constituent of the entry complex.

Most studies to the functional properties of the PIFs were conducted when it was not known yet that these proteins form a complex. Chapter 2 therefore reviewed earlier research data concerning these proteins, now taking into account that they are part of the same complex.

Chapter 3 provided a first clue on why complex formation is important for the individual PIFs. Our initial studies with pif deletion mutant viruses were hampered by the disappearance of not only the targeted, but also non-targeted PIFs, when the OBs were produced in insect larvae, while the various PIFs were all detectable for the wild type virus. However, those remaining PIFs were found again in these mutant viruses when the larval derived OBs were treated with heat prior to ODV isolation, or when the OBs were produced in cultured insect cells. These observations showed that proteases from the host, which had been reported to be co-occluded in the OBs, are able to degrade PIF proteins when complex formation is interrupted, as was the case in the various pif deletion mutant viruses. Analyses of mutant ODVs that lack one of the loosely associated PIFs, PIF0 or PIF6, but are still able to form the stable core, showed that the PIFs in the stable core were slightly more resistant to proteolytic degradation than the same PIFs in monomeric form. However, when the stable core lacks PIF4 and a smaller complex is formed by PIF1, 2 and 3, this resistance was completely lost. It was concluded that complex formation by PIF proteins is required to resist proteolytic degradation by host proteases, which 
might be important to cope with the hostile environment in the larval midgut that contains lots of digestive enzymes.

Previous research in our laboratory had identified AC108 as a PIF1 interaction partner in a coimmunoprecipitation study, indicating that this protein might be involved in oral infectivity as well. Chapter 4 demonstrated that mutation of the ac108 gene from the AcMNPV genome completely abolished the oral infectivity of ODVs, which was recovered when this gene was repaired. Furthermore, protein analysis of the ac108 mutant virus and an HA-tagged repair virus showed that AC108 is a loosely associated but nevertheless an essential component of the ODV entry complex. This protein was therefore designated as PIF9.

The biological function of PIF9 was further investigated in Chapter $\mathbf{5}$ by the generation of fluorescently labelled ODVs of the pif9 mutant. The ODVs were made fluorescent by the incorporation of eGFP:VP39 fusion proteins in the nucleocapsids. The fluorescent ODVs were then combined with isolated midgut cells of Spodoptera exigua larvae and monitored by confocal microscopy in a time lapse experiment. It was observed that in absence of PIF9, the ODVs were still able to bind to the midgut cell brush border, but that the nucleocapsids failed to enter the cell, in contrast to fluorescent ODVs that have all PIFs. Fluorescent ODVs were also generated for a pif3 deletion mutant as it had been shown by others with R18 dequenching assays that this (complex deficient) mutant is able to bind and fuse with brush border membrane vesicles as the wild type virus, but nevertheless fails to establish a midgut infection. Our analysis with the confocal microscope showed that this mutant displays the same phenotype as the pif9 deletion mutant virus: ODV binding occurred, but entry of the nucleocapsids was blocked. As ODVs of the pif3 deletion mutant had been reported to be fusogenically active in the R18 de-quenching assays, we proposed a two-step fusion process, in which first the outer leaflets of the lipid bilayers fuse and subsequently the inner leaflets. Fusion of the outer leaflets had been shown by others with other mutant viruses to result in R18 dequenching as well and might explain why despite the observed fusogenic activity, infection of the midgut fails with ODVs that lack PIF3. This PIF might therefore instead of important for translocation of the nucleocapsids as proposed by others be important to complete the fusion process by aiding fusion of the inner leaflets of the lipid bilayers.

PIF1 and 2 had been reported to be important for ODV binding and were later also shown to be crucial for the formation of the ODV entry complex. It was asked whether these two PIFs are directly or indirectly involved in interaction with the host. As ODV binding is one of the viral 
host range determinants and most baculoviruses have a narrow host range, it was hypothesized that the more variable C-terminal parts of PIF1 and 2 are important for host interaction, while the highly conserved $\mathrm{N}$-terminal parts are needed for complex formation. Chapter $\mathbf{6}$ aimed to distinguish those two functions by analysing a series of mutant ODVs with C-terminally truncated versions of one of those PIFs. Limited C-terminal truncation of either PIF1 or 2 abolished formation of the entry complex, while the stable core was still being formed. These findings were supported by co-immunoprecipitation studies with PIF1 antiserum. These studies confirmed the interaction between PIF1 and 2 in the core of the truncation mutants, but the interactions between PIF1 and the largest loosely associated PIFs (PIF0 and 8) were lost in those mutants. This shows that the truncated parts of PIF1 and 2 are important for the binding of at least PIF0 and 8 to the stable core of the ODV entry complex. It was also found that in absence of PIF4, 6 or 8, PIF0 was still able to bind the core or the smaller complex formed by PIF1, 2 and 3, while the other loosely associated PIFs requires each other's presence to bind the entry complex. The lost ability to form the entry complex in the truncation mutants was accompanied by a severely hampered oral infectivity in S. exigua larvae. However, some larvae incidentally got infected by one of the truncation mutants despite abolished complex formation, which also had been reported by others with various other pif mutant viruses. It was therefore speculated that although the ODV entry complex is the main determinant for the infectivity of ODVs, additional factors are present in the ODV that is able to incidentally cause infections in absence of the entry complex.

The context in which the PIFs function was investigated in Chapter 7 by construction of a socalled PIF-interactome. Three different loosely associated components of the entry complex (PIF6, 8 and 9) were immunoprecipitated and the co-immunoprecipitated proteins from the ODV envelope were identified by mass spectrometry. These experiments were also performed for PIF5, the only PIF that is not part of the entry complex. These analyses revealed that the PIFs interact with a plethora of viral proteins with a wide variety of functions, from nucleocapsid assembly to OB formation. Immunoprecipitation of PIF5 also resulted in the coprecipitation of two components of the entry complex, PIF0 and 1. This indicated that PIF0 and 1 are not only present in the ODV envelope as part of the entry complex, but also occur outside the complex where these proteins interact with PIF5. ODV-E66 was also identified as a PIF5 interaction partner and this protein was also found after the immunoprecipitation of PIF6 and 9. These findings are in line with other studies that showed that ODV-E66 is important for the ODV oral infectivity and that this protein interact with various PIF proteins. This study shows 
that the PIFs not only interact with each other to form the entry complex, but are part of a network of protein interactions in ODVs. The biological significance of these interactions for midgut infection remains enigmatic.

Chapter 8 discussed the findings in a broader perspective and compared the ODV entry complex in baculoviruses with two other known viral entry complexes that occur in members of the Herpesviridae and Poxviridae. Also an infection model was presented, in which, based on the studies of this PhD thesis and literature data, the roles of the PIFs were integrated. At last, possible directions for future research were discussed. 


\section{Samenvatting}

Insectenlarven worden door baculovirussen geïnfecteerd als ze met polyeders besmet bladmateriaal eten. Polyeders zijn door het virus gevormde deeltjes die bestaan uit een veelvlakvormige matrix van gekristalliseerd eiwit (polyhedrine of granuline) waarin de infectieuze virusdeeltjes zijn ingebed. De polyeders vallen na consumptie uiteen in het alkalische milieu van de middendarm van de insectenlarf, waardoor de virusdeeltjes vrijkomen en vervolgens de darmepitheelcellen infecteren. Om deze cellen te kunnen infecteren bevat het lipide membraan van de virusdeeltjes een specifieke groep eiwitten genaamd per os infectiefactoren (PIFs). Aan het begin van dit onderzoek waren er acht verschillende PIFs bekend, waarvan er zes betrokken zijn bij de vorming van een eiwitcomplex: het PIF-complex. Inmiddels zijn er tien verschillende PIFs bekend, waarvan er negen deel uitmaken van dit complex. Vier PIFs, PIF1, 2, 3 en 4, vormen een stabiele kern van dit complex dat bestand is tegen behandeling met denaturerende en reducerende stoffen. De overige vijf PIFs (PIF0, PIF6, 7, 8 en 9) binden met lagere affiniteit aan het complex en kunnen alleen onder nietdenaturerende omstandigheden in het complex gevonden worden. PIF5 is het enige PIF-eiwit dat geen deel uitmaakt van het complex.

De meeste studies naar de biologische functies van de verschillende PIFs bij de infectie van het darmepitheel waren uitgevoerd toen het nog niet bekend was dat ze samen een complex vormen. Deze studies worden daarom in hoofdstuk 2 besproken met in ogenschouw neming van het feit dat dat de PIFs deel uitmaken van hetzelfde eiwitcomplex.

De experimenten die in hoofdstuk 3 beschreven staan gaven voor het eerst een idee waarom de PIFs eigenlijk een complex vormen in het ODV-membraan. Uit eiwitanalyses van mutantvirussen met een deletie van één van de pif-genen bleek namelijk dat niet alleen dat betreffende PIF-eiwit afwezig was, maar dat ook de andere PIF-eiwitten, waarvan het gen intact is, niet gedetecteerd konden worden, als het virus was geproduceerd in insectenlarven. De overige PIFs werden echter wel gevonden als dezelfde virusmutanten waren geproduceerd in gekweekte insectencellen of als de in larven-geproduceerde polyeders waren verhit vóórdat de ingebedde virusdeeltjes gezuiverd werden met behulp van een alkalische buffer. Deze observaties wijzen erop dat proteasen van de gastheer, die ook worden ingebed in de eiwitmatrix van de polyeders, in staat zijn om individuele PIF-eiwitten af te breken als het complex niet gevormd wordt. Uit analyses van virusmutanten waarin de stabiele kern van het PIF-complex nog gevormd werd, bijvoorbeeld in de afwezigheid van één van de losse 
componenten PIF0 of PIF6, bleek dat de PIFs als onderdeel van de stabiele kern minder snel werden afgebroken dan dezelfde PIF in niet-geassocieerde vorm. Dit effect was echter verdwenen wanneer PIF1, 2 en 3 samen een kleinere kern vormen in afwezigheid van PIF4. Op basis van deze observaties werd geconcludeerd dat de PIF-eiwitten een complex vormen om bestand te zijn tegen de proteolytische activiteit van gastheerproteasen, wat mogelijk belangrijk is voor behoud van de biologische activiteit van de virusdeeltjes in de middendarm alwaar veel digestieve proteasen aanwezig zijn.

Voorgaand onderzoek in dit laboratorium identificeerde het AC108-eiwit als interactiepartner van PIF1. Dit duidde er op dat dit virale eiwit mogelijk ook betrokken is bij de perorale infectie van de gastheer door het virus. Hoofdstuk 4 liet zien dat een virus met een mutatie van het ac108-gen niet meer infectieus was via de orale route. Perorale infectie was echter weer volledig hersteld als dit gen gerepareerd was. Uit eiwitanalyses bleek dat dit eiwit een essentieel component is van het PIF-complex; d.w.z. dat in afwezigheid van AC108 het PIF-complex niet gevormd werd. Na aanleiding van deze experimenten werd AC108 aangeduid als PIF9.

De biologische functie van PIF9 werd verder onderzocht in hoofdstuk 5 met fluorescerende virusdeeltjes van de pif9 virusmutant. De virusdeeltjes werden fluorescerend gemaakt door het inbouwen van een eGFP:VP39 fusie-eiwit in de capsiden van het virus. De fluorescerende virusdeeltjes werden vervolgens gemengd met geïsoleerde epitheelcellen uit de middendarm van de gastheer (Spodoptera exigua larven) en geobserveerd met een confocale laserfluorescentie microscoop. Hieruit bleek dat in afwezigheid van PIF9 de virusdeeltjes nog wel in staat waren om aan de microvilli van de darmepitheelcellen te binden, maar dat de capsiden de cel niet konden binnendringen in tegenstelling tot die van het wildtype virus. Dit experiment werd ook uitgevoerd met virusmutanten waarin het PIF3-eiwit ontbrak. Ondanks dat het PIF-complex ook in deze mutant niet gevormd werd, bleek uit eerdere studies naar membraanfusie waarin het membraan van de virusdeeltjes zijn geladen met een fluorescerende stof (R18), dat deze virusmutant desondanks instaat is het virale membraan te kunnen laten fuseren met het membraan van de gastheercel. Echter vond geen infectie van het darmepitheel van de gastheer plaats. Uit onze microscopische analyses met fluorescerende capsiden bleek dat deze virusmutant hetzelfde fenotype had als de hiervoor beschreven pif9 virusmutant: wel binding aan de microvilli maar geen binnendringing van de capsiden. De uitkomsten van deze twee studies ondersteunen een model waarin fusieproces bestaat uit twee stappen, waarbij eerst de buitenlagen van de twee membranen fuseren en daarna de binnenlagen. Hieruit werd 
geconcludeerd dat PIF3 mogelijk belangrijk is voor het fuseren van de binnenlagen van de membranen tijdens de infectie van de darmepitheelcellen.

PIF1 en 2 zijn belangrijk voor de bindingscapaciteit van de virusdeeltjes aan de darmepitheelcellen van de gastheer en bleken later ook essentieel voor de vorming van het PIFcomplex. Dit leidde tot de vraag of deze twee PIF-eiwitten direct of indirect met de gastheercellen interacteren. Omdat de bindingscapaciteit van de virusdeeltjes medebepalend is of het virus wel of niet bepaalde insectensoorten kan infecteren en de meeste baculovirussen slechts één of enkele insectensoorten kunnen infecteren werd verondersteld de meer variabele C-terminale delen van PIF1 en 2 betrokken zijn bij gastheerinteractie en dat de sterk geconserveerde N-terminale delen betrokken zijn bij de vorming van het PIF-complex. In hoofdstuk 6 werd deze hypothese getoetst door een panel virusmutanten te analyseren die Cterminaal verkortte PIF1 - of 2-eiwitten bevatten. Het ontbreken van zeer kleine delen van de C-terminale uiteinden van PIF1 of 2 resulteerde al in een deficiëntie om het PIF-complex te vormen. De stabiele kern werd echter nog wel gevormd in deze virusmutanten. Deze bevindingen werden ondersteund door co-immunoprecipitatie studies met antiserum tegen PIF1, waaruit bleek dat na afknotting van PIF1 of 2 deze twee PIFs nog aan elkaar konden binden, maar dat de losse componenten van het PIF-complex (PIF0 en 8) niet meer direct of indirect aan PIF1 konden binden. Blijkbaar zijn de afgeknotte C-terminale uiteinden van PIF1 en 2 belangrijk voor de binding van tenminste twee losse componenten van het PIF-complex, PIF0 en 8, aan de stabiele kern van het PIF-complex. Deze studies lieten ook zien dat in afwezigheid van PIF4, 6 of 8, PIF0 zich onafhankelijk aan de stabiele kern kan binden, terwijl de andere losse componenten van het PIF-complex elkaars aanwezigheid nodig hebben om het complex te vormen. De deficiëntie om het PIF-complex te vormen na afknotting van PIF1 of 2 ging gepaard met een sterke afname van de perorale infectie van S. exigua larven. Enkele larven bleken echter geïnfecteerd te zijn door de virusmutant, wat ook beschreven is in de literatuur voor diverse andere baculovirus pif-mutanten. Op basis hiervan werd gespeculeerd dat naast het PIF-complex ook andere virale factoren betrokken zijn bij de infectie van de middendarmcellen.

Om te bepalen in welk onderling verband de PIF-eiwitten functioneren in het virale membraan is er in hoofdstuk 7 een zogenaamde "PIF-interactome" gereconstrueerd, waarin de interactiepartners van verschillende PIFs in kaart zijn gebracht. Hiervoor zijn drie losse componenten van het PIF-complex, PIF6, 8 en 9, geprecipiteerd en zijn de interactiepartners van deze eiwitten geïdentificeerd met massaspectrometrie. Deze analyses waren ook uitgevoerd 
na precipitatie van PIF5, het enige PIF-eiwit dat geen deel uitmaakt van het complex. PIF0 en 1 werden als enige twee componenten van het PIF-complex geïdentificeerd als interactiepartner van dit eitwit, wat erop duidt dat deze twee PIFs in het viraal membraan ook buiten het PIFcomplex voorkomen en dan aan PIF5 binden. ODV-E66 bindt ook aan PIF5 en dit eiwit werd ook gevonden na precipitatie van PIF6 en 9. Deze resultaten komen overeen met studies van anderen waaruit bleek dat ODV-E66 belangrijk is voor de perorale infectie en hierbij interacteert met diverse PIF-eiwitten. Daarnaast bleek uit deze analyses dat de verschillende PIF-eiwitten, met name PIF9, interacteren met vele andere virale (niet-PIF) eiwitten in het membraan van de virusdeeltjes. Deze eiwitten hebben uiteenlopende functies: van vorming van de capsiden tot inbedding van de virusdeeltjes in de eiwitmatrix van de polyeders. De biologische functie van deze interacties zijn echter nog onduidelijk.

De resultaten van dit onderzoek werden in een bredere context bediscussieerd in hoofdstuk 8 , waarin het PIF-complex van baculovirussen werd vergeleken met twee andere virale eiwitcomplexen in virussen die behoren tot de Herpesviridae en Poxviridae en essentieel zijn voor de infectie van gastheercellen. Daarnaast werd er een infectiemodel gepresenteerd, waarin de resultaten van onze studie en uit de literatuur geïntegreerd werden. Tot slotte werden mogelijkheden voor verder onderzoek voorgesteld en bediscussieerd. 


\section{Acknowledgements - Dankwoord}

In maart 2015 begon dit avontuur dat na ruim 4.5 jaar hard werken resulteert in de totstandkoming van dit proefschrift. Deze tijd is ongelofelijk snel voorbij gegaan en ik had deze ervaring voor geen goud willen missen. $\mathrm{Nu}$ rest het mij om alle mensen die in deze periode een belangrijke rol hebben gespeeld te bedanken.

Allereerst wil ik Monique en Jan bedanken voor het gegeven vertrouwen en de vrijheid die jullie mij gaven om naar eigen inzicht invulling aan dit onderzoek te geven. Ik weet nog dat ik bij jullie op sollicitatiegesprek kwam en daardoor mijn interesse in het project verder aanwakkerde en dat ik na afloop dacht: hieraan wil ik werken. Gelukkig dachten jullie er ook zo over ondanks dat ik geen enkele virologische ervaring had en begon ik een ander halve maand later aan mijn PhD-project over het baculovirus per os infectie complex.

Monique, ik wil jou in het bijzonder bedanken voor jouw aanstekelijke enthousiasme en inspiratie, en dat je deur altijd openstond als ik even iets wilde weten. Het tempo waarin je mijn stukken nakeek is zeer bewonderenswaardig en zorgde voor een vloeiend verloop van het publicatieproces van de verschillende artikelen. Daarnaast bracht je mij altijd in contact met de juiste mensen als ik specifieke expertise nodig had.

Jan, afgelopen jaren hebben we intensief samengewerkt, waarbij je mij hebt geleerd om kritisch te denken en vooral naar simpele oplossingen te zoeken. Daarnaast bewonder ik jouw taalgevoel, waarvan ik tijdens het schrijven van de verschillende stukken bijzonder veel van heb opgestoken. Ook denk ik met veel plezier terug aan de practica van het vak Fundamentals and Applied Virology, die we gegeven hebben met Dick Lohuis en wijlen Hanke Bloksma. Dat waren gezellige perioden waarvoor ik het andere werk graag even aan de kant zette. Naast het harde werken en de wetenschappelijke discussies denk ik ook met veel plezier terug aan de gezellige lunches (en natuurlijk de ambachtelijke frietjes) en onze discussies over de actualiteit waarbij we de controversiële onderwerpen niet uit de weg gingen. Kortom, jij hebt mijn $\mathrm{PhD}$-periode kleur gegeven. Bedankt daarvoor!

Vera, hartelijk bedankt voor de raad en daad die je mij afgelopen jaren hebt gegeven. Ik bewonder je scherpe oog voor detail dat goed van pas kwam als we de presentaties gingen oefenen voor de SIP-meeting. Ook aan het geven van de practica pestmanagement denk ik met plezier terug. 
Els, jij staat altijd voor iedereen klaar en zorgde ervoor dat alles op het lab ordelijk verliep. Daarnaast zorgde je voor de reagentia en het kweken van de cellen en insecten. Zonder jouw ondersteuning was dit werk dan ook niet mogelijk geweest! Ook was je altijd in voor een praatje en zorgde je voor een goede sfeer op het lab. Bedankt voor de fijne samenwerking.

Han, you were my buddy in the lab and helped me a lot when I started my research at virology. You were always there when I had a question, even when it was already late. It was a pleasure to work with you and I wish you all the best in your future career!

Simone: it was a pleasure to work with you in the lab, especially during the practicals of Fundamentals and Applied Virology and with the organization of the international dinner. You have a great attitude and I will never forget the radio-show "Danske Bingo" that you like to listen in the lab. As I don't speak Danish, you always explained me the jokes the Discjockeys made so I can appreciate the Danish sense of humor. Also the trip to Valencia for the SIP-meeting was a lot of fun. I also want to thank you for being my Paranymph. Tak igen for alt og held og lykke med resten af din ph.d.!

I also like to thank my students, Kevin, Coen, Fabiola, Alexander, Maarten, Felix, Wendy, Jorn, Priyanka and Justin, who participated in my PhD-project for their efforts. It was a pleasure to supervise you. I have learned a lot from being your supervisor and this work would not be possible without your accomplishments! I hope you have enjoyed it as much as I did and I wish you all the best for your future careers.

It is very important for me to work in a vivid environment and my time at virology would not be the same without the people who together shape the virology department: Andre, Christina, Corien, Corinne, Dick, Fengqiao, Gabriela, Giel, Gorben, Haidong, Irene Meki, Irene van Grindsven, Jelke, Just, Magda, Marcel, Marleen, Mia, René, Richard, Sandra and Tessy. Thanks a lot for the nice coffee breaks (with lots of pie) and the fun we have had during the international dinners and Labtrips!

Het laatste hoofdstuk van dit proefschrift met de proteomics analyse was niet mogelijk geweest zonder Sjef Boeren van het Laboratorium voor Biochemie. Sjef, hartstikke bedankt voor het meedenken met dit experiment, de massa spectrometrie metingen en de analyses.

Ik wil graag Claudius van de Vijver en Lennart Suselbeek van de PE\&RC graduate school bedanken voor de leerzame PE\&RC dagen en weekenden. Ik vond het altijd leuk om hierbij aanwezig te zijn. Ook wil ik de PE\&RC graduate school bedanken voor het beschikbaar 
stellen van strategische middelen wat mij instaat stelde extra onderzoek te doen in samenwerking met het Laboratorium voor Biochemie.

I also want to thank the members of the virus division of the Society of Invertebrate Pathology (SIP) for giving me the opportunity to share my thoughts and ideas with international experts. I was able to attend at four of those meetings, of which the first was in Tours in 2016. Each year I looked forward to attend these meetings and it was an honour to serve as student representative for two years. I especially want to thank Prof. Dr Zhihong $\mathrm{Hu}$ (Rose), Dr Manli Wang, Dr David Theilmann and Dr Martin Erlandson for their interest and fresh views on my work, which really helped my work forward.

Tot slotte wil ik mijn steun en toeverlaat bedanken: Basia, toen ik begon aan dit traject mijn vriendin en nu mijn vrouw. Jij hebt mij altijd gesteund als ik het druk had en in het weekend nog moest werken. Daarbovenop heb je ook nog veel geregeld voor onze bruiloft (wat een onvergetelijk dag was) en heb je de verbouwing van ons eerste eigen huis in goede banen geleid. Daarom mag je niet aan mijn zijde ontbreken als paranymph bij de verdediging van dit proefschrift. Schat, bedankt voor de steun van afgelopen jaren en ik vind het geweldig de rest van mijn leven met je te mogen delen.

\section{Bob Boogaard}

Vlaardingen

29 oktober 2019 
- 154 - 


\section{About the author}

Bob Boogaard was born on April the $3^{\text {rd }} 1987$ in Zeist. After finishing primary and secondary school, he studied Biomedical Sciences at the VU University in Amsterdam and obtained his BSc-degree in 2012 by completing his thesis entitled: "The effects of small molecule TMC207 on mortality and ATP homeostasis in Mycobacterium bovis BCG". He continued his studies with a Master in Biomolecular Sciences, also at the VU University, in which he gained more fundamental knowledge on molecular mechanisms underlying health and disease states. In this track, he

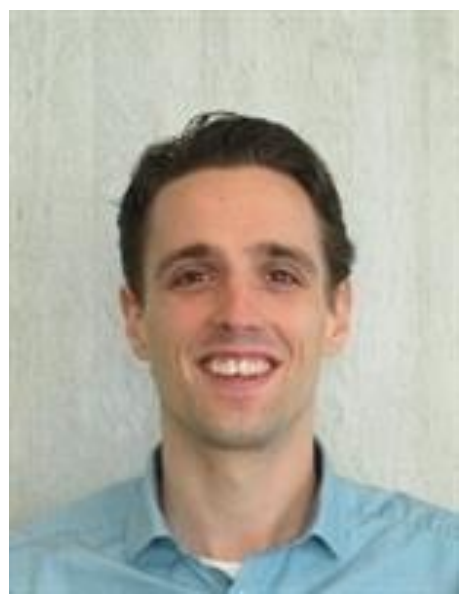
followed two internships, in which he studied paracetamol-induced starvation responses in yeast cells, at the Molecular Toxicology department of the VU, and the constitutive signaling activity of adhesion-GPCR GPR64 at the department of Medicinal Chemistry at Leiden University. In 2015, he started his $\mathrm{PhD}$-project on the molecular action of a protein complex that is formed by baculovirus per os infectivity factors (PIFs), which is essential for the infection of moths and butterflies via the oral route. In this project, he studied the composition of this complex and obtained more insight on the protein interactions that are required to form such a complex. He also studied with which other viral proteins the PIFs interact in the virus envelope and performed functional studies with fluorescently labeled pif mutant viruses on isolated midgut cells. In addition to doing research, he was also involved in education as PGOtutor in the course Cell Biology and Health and as practical assistant in the course Fundamental and Applied Virology. Furthermore, he supervised ten thesis students, who participated in his research. Bob was also a student representative of the virus division of the Society of Invertebrate Pathology (SIP), in which he organized and moderated student meetings during the annual SIP-meetings. After finishing this PhD-study, he will start as a postdoctoral researcher in the group of Prof. Dr Ron Fouchier at the Erasmus MC to study the aero stability of various influenza viruses under supervision of Dr Sander Herfst. 


\section{Account}

Boogaard, B., Van Lent, J. W. M., Theilmann, D. A., Erlandson, M. A., \& van Oers, M.

M. (2017). Baculoviruses require an intact ODV entry-complex to resist proteolytic degradation of per os infectivity factors by co-occluded proteases from the larval host. Journal of General Virology, 98, 3101-3110.

Boogaard, B., Van Oers, M. M., \& Van Lent, J. W. M. (2018). An advanced view on baculovirus per os infectivity factors. Insects, 9, 84 .

Boogaard, B., Evers, F., van Lent, J. W. M., \& van Oers, M. M. (2019). The baculovirus AC108 protein is a per os infectivity factor and a component of the ODV entry complex. Journal of General Virology, 100, 669-678. 


\section{PE\&RC Training and Education Statement}

With the training and education activities listed below the PhD candidate has complied with the requirements set by the C.T. de Wit Graduate School for Production Ecology and Resource Conservation $(P E \& R C)$ which comprises of a minimum total of 32 ECTS ( $=22$ weeks of activities)

\section{Review of literature (6 ECTS)}

- An advanced view on baculovirus per os infectivity factors

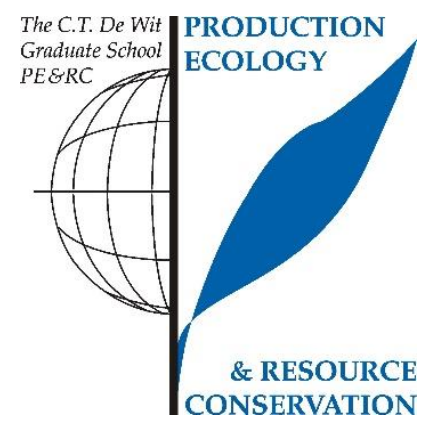

\section{Post-graduate courses (3.7 ECTS)}

Advanced proteomics; VLAG (2015)

Advanced statistics course design of experiments; PE\&RC (2017)

Basic transmission electron microscopy; WUR (2019)

\section{Invited review of (unpublished) journal manuscript (2 ECTS)}

- Applied and Environmental Microbiology: second type of resistance against CpGV

- Journal of General Virology: microscopic examination of AcMNPV infection in the larval midgut

Deficiency, refresh, brush-up courses (2.3 ECTS)

- Molecular virology; Virology (2015)

- $\quad$ Better Safe Than Sorry (BSTS); Jakob Laegdsmand (2015)

\section{Competence strengthening / skills courses (4 ECTS)}

Competence assessment; WGS (2016)

- $\quad$ Essentials of writing and presenting; WGS (2016)

- Scientific writing; WGS (2017)

- $\quad$ Scientific artwork, vector graphics and images; WUR (2018)

PE\&RC Annual meetings, seminars and the PE\&RC weekend (2.7 ECTS)

- PE\&RC First years weekend (2015)

- PE\&RC Day (2015-2017)

- $\quad$ PE\&RC Midterm weekend (2017)

\section{Discussion groups / local seminars / other scientific meetings (4.5 ECTS)}

- Wageningen evolution and ecology seminars (2015-2018)

- Dutch annual virology symposium (2015-2019)

International symposia, workshops and conferences (15 ECTS)

- Viruses: host pathogen interactions; poster presentation (2016)

- Society of invertebrate pathology; oral and poster presentations (2016-2019)

- $\quad$ European congress of virology; poster presentation (2019)

Lecturing / supervision of practicals / tutorials (4.5 ECTS)

Cell biology and health; PGO (2016-2018)

- $\quad$ Fundamental and applied virology (2016-2019)

\section{Supervision of MSc students}

Construction and characterisation of PIF truncation mutant viruses

Identification of a putative new PIF protein in AcMNPV

- Monitoring of fluorescent virus particles on isolated midgut cells 
The research described in this thesis was financially supported by The Netherlands Organisation of Scientific Research (NWO), grant number: 824.14.16, and by strategic means of the Graduate School for Production Ecology and Resource Conservation (PE\&RC).

Cover design: Electron microscopic picture of a baculovirus occlusion body in the midgut of a Spodoptera exigua larva, made by Dr Jan W. M. van Lent at the Wageningen Electron Microscopy Center.

Printed by GVO drukkers \& vormgevers, Ede, The Netherlands 
